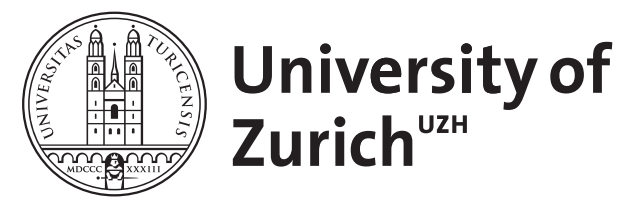

\title{
La réalisation étale et les opérations de Grothendieck
}

\author{
Ayoub, J
}

\begin{abstract}
In this article, we construct étale realization functors defined on the categories DAét $(\mathrm{X}, \Lambda)$ of étale motives (without transfers) over a scheme X. Our construction is natural and relies on a relative rigidity theorem à la Suslin-Voevodsky that we will establish first. Then, we show that these realization functors are compatible with Grothendieck operations and the "nearby cycles" functors. Along the way, we prove a number of properties concerning étale motives.
\end{abstract}

DOI: https://doi.org/10.24033/asens.2210

Posted at the Zurich Open Repository and Archive, University of Zurich ZORA URL: https://doi.org/10.5167/uzh-97937

Journal Article

Originally published at:

Ayoub, J (2014). La réalisation étale et les opérations de Grothendieck. Annales Scientifiques de l'Ecole Normale Superieure, 47(1):1-145.

DOI: https://doi.org/10.24033/asens.2210 


\title{
LA RÉALISATION ÉTALE ET LES OPÉRATIONS DE GROTHENDIECK
}

\author{
par
}

Joseph Ayoub

\begin{abstract}
Résumé. - Dans cet article, nous construisons des foncteurs de réalisation étale définis sur les catégories $\mathbf{D A}^{\text {ét }}(X, \Lambda)$ des motifs étales (sans transferts) au-dessus d'un schéma $X$. Notre construction est naturelle et repose sur un théorème de rigidité relatif à la Suslin-Voevodsky que nous devons établir au préalable. Nous montrons ensuite que ces foncteurs sont compatibles aux opérations de Grothendieck et aux foncteurs « cycles proches ». Au passage, nous démontrons un certain nombre de propriétés concernant les motifs étales.
\end{abstract}

Abstract. - In this article, we construct étale realization functors defined on the categories $\mathbf{D A} \mathbf{A}^{\text {et }}(X, \Lambda)$ of étale motives (without transfers) over a scheme $X$. Our construction is natural and relies on a relative rigidity theorem à la Suslin-Voevodsky that we will establish first. Then, we show that these realization functors are compatible with Grothendieck's operations and the "nearby cycles" functors. Along the way, we prove a number of properties concerning étale motives.

\section{Table des matières}

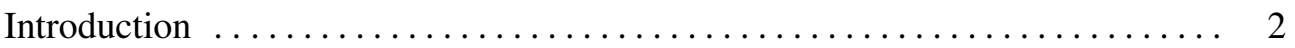

1. Semi-séparation pour les 2-foncteurs homotopiques stables $\ldots \ldots \ldots \ldots \quad 5$

2. Vérification de la condition $\left(\mathbf{S S}_{p}\right)$ et orientation $\ldots \ldots \ldots \ldots \ldots \ldots \ldots \ldots \ldots$

3. Le 2-foncteur homotopique stable $\mathbf{D A}{ }^{\text {ét }}(-, \Lambda) \ldots \ldots \ldots \ldots \ldots \ldots \ldots$

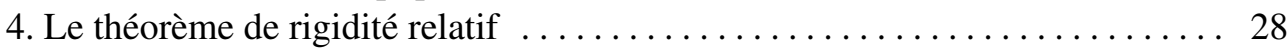

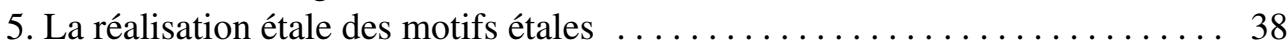

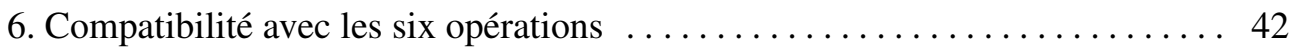

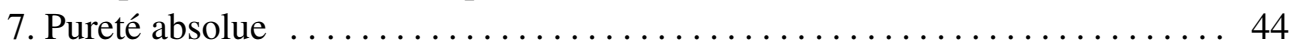

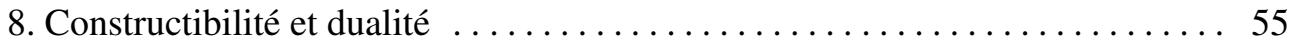

9. La réalisation étale des motifs étales (suite) $\ldots \ldots \ldots \ldots \ldots \ldots \ldots \ldots \ldots 6 . \ldots \ldots$

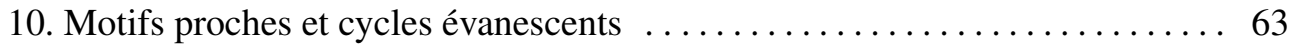

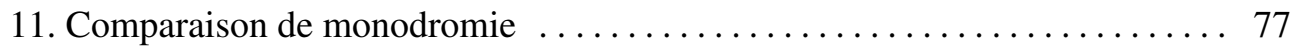

Annexe A. Changement de coefficients $\ldots \ldots \ldots \ldots \ldots \ldots \ldots \ldots \ldots \ldots . \ldots \ldots$

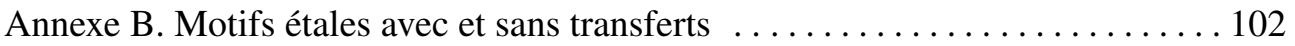

Annexe $\mathbf{C}$. Vérification de la condition $\left(\mathbf{S S}_{p}\right)$ : deuxième méthode $\ldots \ldots \ldots 108$

Références ..........................................113

Classification mathématique par sujets (2000). - 13D09, 14F05, 14F20, 14F42, 19D99.

Mots clefs. - Motif, cohomologie étale, réalisation étale, six opérations de Grothendieck, formalisme des cycles proches. (Motives, étale cohomology, étale realization, Grothendieck six operations, nearby cycles formalism.)

L'auteur a bénéficié du soutien partiel du Fond National Suisse de la Recherche Scientifique (NSF), projet no. 200021-124737/1. 


\section{Introduction}

Soient $k$ un corps parfait et $n$ un entier inversible dans $k$. On note $\mathbf{D M}(k, \Lambda)$ la catégorie des motifs de Voevodsky sur $k$ à coefficients dans un anneau commutatif $\Lambda$. Le théorème de rigidité de Suslin-Voevodsky (cf. [31, Th. 7.20 and Th. 9.35]) fournit une construction simple et naturelle de la réalisation étale à coefficients dans $\mathbb{Z} / n \mathbb{Z}$. C'est la suivante. Étant donné un motif $M \in \mathbf{D M}(k, \mathbb{Z})$, on peut former le motif étale à coefficients finis aét $(M \otimes \mathbb{Z} / n \mathbb{Z}) \in \mathbf{D M}^{\mathbf{e}^{\mathrm{et}}}(k, \mathbb{Z} / n \mathbb{Z})$ obtenu en tensorisant $M$ par $\mathbb{Z} / n \mathbb{Z}$ et en faisceautisant pour la topologie étale. D'après le théorème de rigidité de Suslin-Voevodsky on dispose d'une équivalence de catégories $L \iota^{*}: \mathbf{D}\left(\mathbf{S h}_{\text {ét }}(\mathrm{Et} / k, \mathbb{Z} / n \mathbb{Z})\right) \simeq$ $\mathbf{D M}^{\text {ét }}(k, \mathbb{Z} / n \mathbb{Z})$. L'image de aét $(M \otimes \mathbb{Z} / n \mathbb{Z})$ par un quasi-inverse de $L \iota^{*}$ est un complexe de faisceaux de $\mathbb{Z} / n \mathbb{Z}$-modules sur Et $/ k$, le petit site étale de $k$. C'est donc essentiellement un complexe de $\mathbb{Z} / n \mathbb{Z}$-modules muni d'une action continue du groupe de Galois absolu de $k$. Ce complexe est la réalisation étale de $M$.

L'objectif de cet article est d'obtenir une version relative de la construction esquissée ci-dessus, et de vérifier que les foncteurs de réalisation qu'on obtient sont compatibles aux opérations de Grothendieck et aux foncteurs « cycles proches ». Pour ce faire, nous devons établir au préalable une version relative du théorème de rigidité de Suslin-Voevodsky. Plus précisément, nous devons

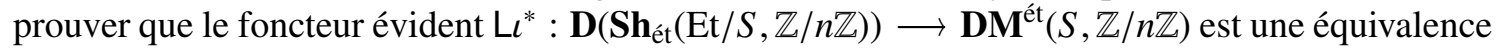
de catégories pour des schémas $S$ suffisamment généraux. Malheureusement, une preuve directe semble difficile et la tentative naïve de ramener le cas général à celui connu où la base est le spectre d'un corps parfait est vouée à l'échec puisque l'axiome de localité pour les catégories de Voevodsky n'est pas disponible dans une généralité suffisante. On est donc naturellement amené à travailler avec les catégories $\mathbf{D} \mathbf{A}^{\text {ét }}(S, \Lambda)$, la variante sans transferts de la catégorie des motifs étales sur $S$. Pour ces catégories, l'axiome de localité est connu (cf. [5, Cor. 4.5.44]) et on verra qu'elles vérifient toutes les propriétés requises et notamment la variante relative du théorème de rigidité de Suslin-Voevodsky. De plus, on a un foncteur évident $\mathbf{D} \mathbf{A}^{\mathrm{ét}}(S, \Lambda) \longrightarrow \mathbf{D M}^{\mathrm{e} t}(S, \Lambda)$ qui est souvent une équivalence de catégories (cf. l'Annexe B). On ne fait donc que gagner en travaillant avec les catégories $\mathbf{D A}{ }^{\text {ét }}(S, \Lambda)$.

Passons en revue le contenu de l'article.

Le théorème de rigidité relatif. - Notre première tâche est d'obtenir une version relative du théorème de rigidité de Suslin-Voevodsky pour les catégories $\left.\mathbf{D A}^{\mathrm{e}} \mathrm{e}_{(-,} \Lambda\right)$. Précisons de quoi il s'agit. Pour un schéma $S$, on dispose d'un foncteur

$$
\mathrm{L}^{*}: \mathbf{D}\left(\mathbf{S h}_{\mathrm{et}}(\mathrm{Et} / S, \Lambda)\right) \longrightarrow \mathbf{D A}^{\mathrm{ét}}(S, \Lambda)
$$

induit par l'inclusion $\mathrm{Et} / S \hookrightarrow \mathrm{Sm} / S$ de la catégorie des $S$-schémas étales dans celle des $S$ schémas lisses. (Ce foncteur est noté $L \hat{\imath}_{S}^{*}$ dans le corps du texte.) L'énoncé optimal d'un théorème de rigidité relatif à la Suslin-Voevodsky serait que le foncteur $L \iota^{*}$ ci-dessus est une équivalence de catégories si $\Lambda$ est une $\mathbb{Z} / n \mathbb{Z}$-algèbre avec $n$ un entier inversible dans $\mathcal{O}_{S}$. C'est ce que nous démontrerons modulo une condition technique sur $S$ qui est toujours vérfiée en pratique. Pour un enoncé précis, on renvoie le lecteur au Théorème 4.1.

Notre preuve du théorème de rigidité relatif utilise la propriété de semi-séparation pour les catégories $\mathbf{D A}{ }^{\text {ét }}(-, \Lambda)$ (cf. le Théorème 3.9). Cette propriété a été introduite dans [4] dans le cadre d'un 2-foncteur homotopique stable général $\mathrm{H}$. On dit que $\mathrm{H}$ est semi-séparé si le foncteur $e^{*}: \mathrm{H}(X) \longrightarrow \mathrm{H}\left(X^{\prime}\right)$ est une équivalence de catégories pour tout $e: X^{\prime} \longrightarrow X$ fini, surjectif et totalement inséparable. Les Sections 1 et 2 sont consacrées à la notion de semi-séparation. On y travaille dans le cadre abstrait d'un 2-foncteur homotopique stable $\mathrm{H}$ sur une base $S$; le but étant d'identifier des conditions simples qui assurent que $\mathrm{H}$ est semi-séparé. On y arrive en deux temps. D'abord, on introduit la condition $\left(\mathbf{S S}_{p}\right)$, avec $p$ un nombre premier, et on montre qu'elle entraîne que $\mathrm{H}$ est semi-séparé si de plus $S$ est le spectre d'un corps parfait de caractéristique $p$ (cf. le Théorème 1.2 pour un énoncé précis). Ceci est l'objectif de la Section 1. La preuve repose d'une manière essentielle sur le formalisme des quatre opérations de Grothendieck comme il a été 
développé dans [4, Chap. 1]. Ensuite, dans la Section 2, on démontre que la condition $\left(\mathbf{S S}_{p}\right)$ est une conséquence de deux autres conditions faciles à imposer, à savoir :

1. $\mathrm{H}$ est $\mathbb{Z}[1 / 2 p]$-linéaire;

2. le foncteur $e^{*}$ est conservatif si $e$ un revêtement étale d'ordre 2 .

C'est le Théorème 2.8 qui s'applique à $\mathbf{D A}{ }^{\text {ét }}(-, \Lambda)$ dès que $\Lambda$ est une $\mathbb{Z}[1 / 2]$-algèbre. Fort heureusement, pour le 2-foncteur homotopique stable $\mathbf{D A} \mathbf{A}^{\text {ét }}(-, \Lambda)$, une méthode directe basée sur des résultats de F. Morel $[33,36]$ permet de vérifier la condition $\left(\mathbf{S S}_{p}\right)$ sans hypothèse sur $\Lambda$; ceci est expliqué dans l'Annexe C.

La preuve proprement dite du théorème de rigidité relatif occupe la Section 4. On démontre d'abord que (1) est une équivalence de catégories lorsque $S$ est le spectre d'un corps de $p$ dimension cohomologique finie pour les nombres premiers $p$ pertinents. C'est le Lemme 4.6. À part la propriété de semi-séparation, un ingrédient essentiel dans la preuve de ce cas particulier est le théorème de rigidité de Röndigs et Østvær [43, Th. 1.1] qu'on adapte aux motifs étales. Le reste de la preuve consiste à se battre pour ramener le cas d'une base générale à celui où la base est le spectre d'un corps; notre outil principal étant le triangle de localisation (cf. [5, Cor. 4.5.44]) dans les catégories $\mathbf{D A} \mathbf{A}^{\mathrm{e}}(-, \Lambda)$.

La réalisation étale et les opérations de Grothendieck. - Une fois que la version relative du théorème de rigidité de Suslin-Voevodsky est démontrée, il est aisé de construire des foncteurs de réalisation étale $\mathfrak{R}_{S}^{\text {ét }}: \mathbf{D} \mathbf{A}^{\text {ét }}(S, \mathbb{Z}) \longrightarrow \mathbf{D}\left(\mathbf{S h}_{\text {ét }}(\mathrm{Et} / S, \mathbb{Z} / n \mathbb{Z})\right)$ par la méthode esquissée au début de l'introduction. Bien entendu, on doit supposer certaines conditions et il est possible d'utiliser des anneaux de coefficients plus généraux. On peut aussi définir une variante $\ell$-adique à la Ekedahl. Ces constructions font l'objet de la Section 5.

Ensuite, on s'intéresse dans la Section 6 aux compatibilités des foncteurs de réalisation étale avec les six opérations de Grothendieck, à savoir les quatre foncteurs $f^{*}, f_{*}, f_{!}$et $f^{!}$associés aux morphismes quasi-projectifs, ainsi qu'aux bifoncteurs $-\otimes-$ et $\mathrm{Hom}(-,-)$. En gros, tout fonctionne bien et on a les isomorphismes de commutation que l'on pense lorsque certaines conditions techniques, souvent satisfaites en pratique, sont supposées.

Enfin, dans la Section 9, on explique comment obtenir des réalisations étales définies sur les motifs à coefficients rationnels. Plus précisément, pour tout nombre premier $\ell$, on définit un foncteur $\Re_{S}^{\text {ét }}: \mathbf{D A}_{\mathrm{ct}}^{\text {ét }}(S, \mathbb{Q}) \longrightarrow \hat{\mathbf{D}}_{\mathrm{ct}}^{\text {ét }}\left(S, \mathbb{Q}_{\ell}\right)$ où $\mathbf{D} \mathbf{A}_{\mathrm{ct}}^{\text {et }}(S, \mathbb{Q})$ est la sous-catégorie des motifs constructibles à coefficients rationnels et $\hat{\mathbf{D}}_{\mathrm{ct}}^{\text {et }}\left(S, \mathbb{Q}_{\ell}\right)$ est la catégorie dérivée des faisceaux $\ell$-adiques sur $S$. Bien entendu, certaines conditions techniques doivent être satisfaites. Nous renvoyons le lecteur à la Section 9 pour les énoncés précis. Ensuite, nous vérifions que ces foncteurs de réalisation commutent aux six opérations de Grothendieck. Si la construction de la réalisation étale à coefficients rationnels est une extension facile des constructions de la Section 5, l'énoncé de cette commutation suppose le théorème de constructibilité des quatre opérations $f^{*}, f_{*}, f_{\text {! }}$ et $f^{!}$(cf. le Théorème 8.10 ), et des opérations $-\otimes-$ et $\underline{\mathrm{Hom}}(-,-)$ (cf. le Théorème 8.12). C'est pour cette raison que la réalisation étale à coefficients divisibles a été repoussée à la suite des Sections 7 et 8 .

Motifs et cycles proches. - Dans la Section 10, on se propose d'étudier la compatibilité de la réalisation étale avec le formalisme des cycles évanescents. Dans la Section 11 on pousse cette étude un cran plus loin en montrant une compatibilité avec, d'une part, l'opérateur de monodromie sur la partie unipotente du motif proche et, d'autre part, l'action de monodromie sur les cycles proches modérés.

Les foncteurs «motif proche » ont été construits et étudiés dans [5, Chap. 3]. Toutefois, une grande partie de la théorie était confinée à la caractéristique résiduelle nulle. Motivés par des applications arithmétiques potentielles, nous nous sommes efforcés dans la Section 10 d'étendre la théorie des motifs proches au-dessus d'un anneau de valuation discrète de caractéristique résiduelle positive. Cette extension est désormais possible grâce à la pureté absolue pour les catégories $\mathbf{D A}^{\text {ét }}(-, \Lambda)$ obtenue dans la Section 7 en se basant sur des résultats de Gabber, de Quillen et de Morel-Voevodsky, et en s'inspirant de la méthode de Cisinski-Déglise [11]. 
Soient $S$ le spectre d'un anneau de valuation discrète hensélien et $f: X \longrightarrow S$ un $S$-schéma quasi-projectif. On note $X_{\eta}$ et $X_{\sigma}$ les fibres génériques et spéciales de $X$. On dispose alors de deux foncteurs

$$
\Upsilon_{f}, \Psi_{f}^{\text {mod }}: \mathbf{D A}^{\mathrm{e} t}\left(X_{\eta}, \Lambda\right) \longrightarrow \mathbf{D A}^{\mathrm{e} t}\left(X_{\sigma}, \Lambda\right)
$$

Si $M \in \mathbf{D A}^{\text {ét }}\left(X_{\eta}, \Lambda\right)$, alors $\Upsilon_{f}(M)$ est la partie du motif proche de $M$ sur laquelle la monodromie est unipotente et $\Psi_{f}^{\bmod }(M)$ est la partie du motif proche de $M$ sur laquelle la monodromie est modérée. Lorsque $S$ est d'égale caractéristique nulle, on retrouve respectivement les foncteurs $\Upsilon_{f}$ et $\Psi_{f}$ de $[\mathbf{5}, \S 3.4$ et $\S 3.5]$. (On fera attention que les foncteurs $\Upsilon_{f}$ ne sont raisonnables qu'à coefficients rationnels alors que les foncteurs $\Psi_{f}^{\bmod }$ se comportent bien en inversant les nombres premiers problématiques.) Lorsque la caractéristique résiduelle de $S$ est non nulle, on construit un troisième foncteur $\Psi_{f}: \mathbf{D A}^{\text {ét }}\left(X_{\eta}, \Lambda\right) \longrightarrow \mathbf{D A}^{\text {ét }}\left(X_{\bar{\sigma}}, \Lambda\right)$ où $X_{\bar{\sigma}}$ est la fibre géométrique au-dessus du point fermé de $S$. Si $M \in \mathbf{D A}^{\text {ét }}\left(X_{\eta}, \Lambda\right)$, alors $\Psi_{f}(M)$ est le motif proche total de $M$.

Sous certaines conditions techniques, nous construisons aussi des isomorphismes de comparaison $\Re_{X_{\sigma}}^{\text {ét }} \circ \Psi_{f}^{\text {mod }} \simeq \Psi_{f}^{\text {mod }} \circ \Re_{X_{\eta}}^{\text {et }}$ et $\Re_{X_{\bar{\sigma}}}^{\mathrm{et}} \circ \Psi_{f} \simeq \Psi_{f} \circ \mathfrak{R}_{X_{\eta}}^{\text {ét }}$ qui font le lien entre le formalisme des cycles évanescents dans le monde motivique et celui en cohomologie étale.

Compléments à $[\mathbf{4}, \mathbf{5}]$. - Tout au long de cet article, le lecteur trouvera des résultats d'un intérêt indépendant de l'objectif poursuivi qui est la construction de la réalisation étale et l'étude des compatibilités avec les six opérations de Grothendieck et les foncteurs cycles proches. Dans la mesure du possible, nous nous sommes efforcés d'inclure des preuves complètes de tous les résultats utilisés. Logiquement parlant, à part le théorème de rigidité de Röndigs et Østvær [43], les techniques de résolution des singularités (de Hironaka, de Jong et Gabber), les travaux de Gabber en cohomologie étale [14] et [25], les travaux de Quillen [38] et de Morel-Voevodsky [37] sur la $K$-théorie algébrique, l'article ne dépend que de $[4,5]$ (pour la théorie des motifs, des six opérations de Grothendieck et du formalisme des motifs proches) et de [2,3] (pour les résultats standards en cohomologie étale). Ainsi, cet article pourrait être considéré comme un complément précieux à $[4,5]$ contenant des résultats spécifiques aux catégories $\mathbf{D A}^{\mathrm{e} t}(-, \Lambda)$. Faisons une liste (non exhaustive) de quelques-uns de ces résultats : la propriété de séparation (cf. le Théorème 3.9), le théorème de rigidité relatif (cf. le Théorème 4.1), la pureté absolue qu'on obtient en s'inspirant de la méthode de Cisinski-Déglise [11] et en combinant des résultats de Gabber, de Quillen et de Morel-Voevodsky (cf. le Théorème 7.4), une extension (suivant une méthode de Gabber) des résultats de constructibilité de $[4, \S 2.2 .2]$ à des schémas de bases plus généraux (cf. le Théorème 8.12), et bien sûr, l'extension du formalisme des cycles évanescents dans le monde motivique au-delà de la caractéristique résiduelle nulle. Pour finir, nous n'avons pas résisté à la tentation d'inclure une extension d'un résultat important de Cisinski-Déglise [11] affirmant que le foncteur évident $\mathbf{D A}^{\text {ét }}(S, \Lambda) \longrightarrow \mathbf{D M}^{\text {ét }}(S, \Lambda)$ est une équivalence de catégories lorsque $S$ est normal et universellement japonais. Dans [11] on devait supposer que $\Lambda$ est une $\mathbb{Q}$-algèbre; notre extension est valable pour des anneaux de coefficients plus généraux. Nous l'obtenons comme une application de notre théorème de rigidité relatif pour $\mathbf{D} \mathbf{A}^{\text {ét }}(-, \Lambda)$.

Un mot sur d'autres constructions. - Il y a au moins deux constructions antérieurs d'une réalisation étale. La première construction est due à Huber [22, 23] qui se restreint aux motifs géométriques de Voevodsky au-dessus d'un corps parfait. La seconde construction est due à Ivorra [26] qui construit sa réalisation sur la catégorie de motifs effectifs et géométriques de Voevodsky sur un schéma de base général. Les constructions de Huber et Ivorra sont géométriques alors que la notre est de nature homologique. Leur réalisation est contravariante : elle envoie le motif d'un $S$-schéma lisse $f: X \longrightarrow S$ sur le complexe $f_{*}(\mathbb{Z} / n \mathbb{Z}) \in \mathbf{D}\left(\mathbf{S h}_{\text {ét }}(\mathrm{Et} / S, \mathbb{Z} / n \mathbb{Z})\right)$. Notre réalisation est covariante : elle envoie le motif de $X$ sur $f_{!} f^{!}(\mathbb{Z} / n \mathbb{Z})$. Nous renvoyons le lecteur à la Proposition 5.9 pour la relation précise entre notre réalisation étale et celle d'Ivorra. 
Commentaires et remerciements. - Cet article contient un certain nombre de résultats techniques et ennuyeux, souvent connus mais parfois dans une généralité insuffisante. Nous prions les spécialistes de nous excuser et nous espèrons que les autres lecteurs trouverons notre présentation utile. Nous avons été guidés par une intuition très simple. C'est la suivante : si on combine le théorème de rigidité de Suslin-Voevodsky [31, Th. 7.20] et le théorème de localité de Morel-Voevodsky [37, Th. 2.21] on doit pouvoir obtenir par un dévissage, éventuellement long et pénible, une version relative de la rigidité. Le problème était que les théorèmes de Suslin-Voevodsky et de MorelVoevodsky appartenaient à des mondes apparentés mais tout de même différents : le premier était valable dans $\mathbf{D} \mathbf{M}^{\text {ét }}(k, \mathbb{Z} / n \mathbb{Z})$ avec $k$ parfait et $n \in \mathbb{N}$ inversible dans $k$, et le deuxième était valable dans les catégories $\mathbb{A}^{1}$-homotopiques des $S$-schémas, voire dans $\mathbf{D A}^{\text {ét }}(S, \Lambda)$. Cette intuition est devenue un «pré-théorème » le jour où j'ai pris connaissance de l'article [43] de Röndigs et Østvær où ils démontrent une forme de rigidité dans $\mathbf{S H}(k)$ pour les extensions de corps algébriquement clos. Dès lors, je « savais » qu'un théorème de rigidité relatif pour $\mathbf{D A}{ }^{\text {ét }}(-, \Lambda)$ était accessible, mais je n'avais aucune intention de me lancer dans ce projet jusqu' au jour où Bruno Kahn m'avait fait savoir qu'il avait besoin d'une réalisation étale qui soit compatible aux six opérations pour des questions liées aux fonctions $L$ de motifs. Or, un théorème de rigidité relatif fournissait une construction d'une réalisation étale pour laquelle les compatibilités avec les six opérations semblaient immédiates. Comme je venais de terminer la rédaction de mon travail sur le groupe de Galois motivique, je me suis dit que je pourrais me changer les idées en écrivant une petite note sur le théorème de rigidité relatif et la réalisation étale. Les détails techniques se sont avérés plus sérieux et la petite note s'est vite transformée en un long article. Je tiens à remercier Bruno Kahn de m'avoir encouragé à entreprendre ce projet et de m'avoir incité à inclure un paragraphe sur les motifs proches en inégale caractéristique. Une partie de cet article a été rédigée pendant un agréable séjour à l'Université d'Oslo où j'ai visité Paul-Arne Østvær. Je le remercie pour son invitation et pour les discussions que nous avions eues sur le théorème de rigidité.

Conseils et remarques pour le lecteur. - Les références à [4] et [5] étant nombreuses, le lecteur est avisé d'en avoir une copie sous la main. Nous utiliserons parfois la terminologie 2-catégorique comme dans [4]. Ainsi, «1-morphisme » et «2-morphisme » sont synonymes de «foncteur» et «transformation naturelle » respectivement. Tous les schémas considérés dans ce texte sont noethériens même si cela est parfois superflu. Ainsi, « schéma » sera synonyme de «schéma noethérien ». La caractéristique d'un anneau $A$ est l'entier naturel qui engendre le noyau de l'unique morphisme d'anneaux $\mathbb{Z} \longrightarrow A$. La caractéristique d'un schéma $S$ est la caractéristique de l'anneau $\mathcal{O}_{S}(S)$. La caractéristique du corps résiduel d'un point de $S$ est appelée une caractéristique résiduelle de $S$. Ainsi, $S$ est de caractéristiques résiduelles nulles si et seulement si $\mathcal{O}_{S}(S)$ est une $\mathbb{Q}$-algèbre. Enfin, lorsque $S$ est local, on conviendra d'appeler la caractéristique résiduelle de $S$ la caractéristique du corps résiduel de l'unique point fermé de $S$.

À plusieurs endroits dans le texte, on peut remplacer les expressions « quasi-projectif » et «projectif » par «séparé de type fini » et «propre ». Souvent, il s'agit d'une extension immédiate et quelquefois, il faudrait se servir du lemme de Chow. À titre d'exemple, nous renvoyons le lecteur à [11] pour une extension du théorème de changement de base [4, Cor. 1.7.18] du cas d'un morphisme projectif à celui d'un morphisme propre; ce type d'argument figure également dans [3, Expo. XII]. Notons aussi que les opérations $f$ ! et $f^{!}$, construites dans [4, Chap. I] pour les morphismes quasi-projectifs, ont été étendues aux morphismes séparés et de type fini dans [11] en suivant la construction de Deligne dans [3, Expo. XVII]. Dans le texte, nous nous bornerons aux $S$-schémas quasi-projectifs pour rester fidèle à l'esprit de $[4,5]$.

\section{Semi-séparation pour les 2-foncteurs homotopiques stables}

On fixe un schéma de base $S$, et on note $\operatorname{Sch} / S$ la catégorie des $S$-schémas quasi-projectifs. Un $S$-morphisme est une flèche de $\mathrm{Sch} / S$. Soit $\mathrm{H}: \mathrm{Sch} / S \longrightarrow \mathfrak{I} \Re$ un 2-foncteur homotopique stable 
au sens de [4, Déf. 1.4.1] (TR étant la 2-catégorie des catégories triangulées). Rappelons les deux notions suivantes (cf. [4, Déf. 2.1.160]).

\section{DEFINITION 1.1}

(a) On dit que $\mathrm{H}$ est semi-séparé si pour tout $S$-morphisme fini, surjectif et totalement inséparable e $: X^{\prime} \longrightarrow X$, le foncteur $e^{*}: \mathrm{H}(X) \longrightarrow \mathrm{H}\left(X^{\prime}\right)$ est conservatif.

(b) On dit que $\mathrm{H}$ est séparé si pour tout $S$-morphisme surjectif $f: Y \longrightarrow X$, le foncteur $f^{*}$ : $\mathrm{H}(X) \longrightarrow \mathrm{H}(Y)$ est conservatif.

$\mathrm{Si} \mathrm{H}$ est semi-séparé, le foncteur $e^{*}$ est une équivalence de catégories pour $e$ fini, surjectif et totalement inséparable (cf. [4, Prop. 2.1.163]). Autrement dit, on ne change rien en remplaçant « conservatif » par « une équivalence » dans la Définition 1.1, (a). Lorsque $S$ est de caractéristiques résiduelles nulles, $\mathrm{H}$ est automatiquement semi-séparé (cf. [4, Rem. 2.1.161]). ${ }^{(1)}$ En général, il est facile de voir que si $\mathrm{H}$ est semi-séparé, alors il vérifie la propriété suivante pour tout nombre premier $p$.

$\left(\mathbf{S S}_{p}\right):$ Notons $q: \mathbb{G}_{S /(p)} \longrightarrow S /(p)$ la projection canonique et $F_{n}: \mathbb{G m}_{S /(p)} \longrightarrow \mathbb{G}_{S /(p)}$ l'endomorphisme de Frobenius relatif donné par l'élévation à la puissance $p^{n}$ (avec $n \in \mathbb{N}$ ). Alors, les endomorphismes de $q_{*} q^{*}$ et $q_{!} q^{!}$induits par $F_{n}$, i.e., les compositions de ${ }^{(2)}$

$$
q_{*} q^{*} \stackrel{\eta}{\longrightarrow} q_{*} F_{n *} F_{n}^{*} q^{*} \simeq q_{*} q^{*} \quad \text { et } \quad q ! q^{!} \simeq q_{!} F_{n !} F_{n}^{!} q^{!} \stackrel{\delta}{\longrightarrow} q_{!} q^{!}
$$

sont des 2-isomorphismes.

Le résultat principal de ce paragraphe affirme que, réciproquement, $\mathrm{H}$ est semi-séparé si $S$ est le spectre d'un corps parfait de caractéristique $p>0$ et si $\mathrm{H}$ vérifie la condition $\left(\mathbf{S S}_{p}\right)$ ainsi qu'une petite condition technique.

TheOREme 1.2 - Soient $k$ un corps parfait de caractéristique $p>0$ et $\mathrm{H}: \operatorname{Sch} / k \longrightarrow \mathfrak{I} R$ un 2-foncteur homotopique stable qui vérifie la condition $\left(\mathbf{S S}_{p}\right)$. On supposera aussi que $\mathrm{H}$ est engendré par la base au sens de [4, Déf. 2.1.155]. Soit e $: X^{\prime} \longrightarrow X$ un morphisme fini, surjectif et totalement inséparable. Alors, le foncteur $e^{*}: \mathrm{H}(X) \longrightarrow \mathrm{H}\left(X^{\prime}\right)$ est une équivalence de catégories. Autrement dit, $\mathrm{H}$ est semi-séparé.

La preuve du Théorème 1.2 ne sera obtenue qu'à la fin de ce paragraphe à la suite d'un certain nombre de réductions et de résultats intermédiaires. La condition $\left(\mathbf{S S}_{p}\right)$ n'interviendra que dans la preuve de la Proposition 1.11 (et de son Corollaire 1.12). Rappelons que $\mathrm{H}$ est engendré par la base si pour tout $k$-schéma quasi-projectif $X$ la catégorie $H(X)$ possède les sommes infinies et coïncide avec sa plus petite sous-catégorie triangulée stable par sommes infinies et contenant les objets de la forme $f_{\sharp} \pi_{Y}^{*} A$ avec $A \in \mathrm{H}(k), f: Y \longrightarrow X$ un morphisme lisse et $\pi_{Y}$ la projection de $Y$ sur $\operatorname{Spec}(k)$. Cette hypothèse ne servira que dans la preuve de la réduction suivante.

Lemme 1.3 - Pour démontrer le Théorème 1.2, il suffit de démontrer la propriété $\mathrm{P}(e)$ ci-dessous pour tout $k$-morphisme fini, surjectif et totalement inséparable e $: X^{\prime} \longrightarrow X$.

$\mathrm{P}(e)$ : Le 2-morphisme $\eta: \pi_{X}^{*} \longrightarrow e_{*} e^{*} \pi_{X}^{*}$, induit par l'unité de l'adjonction $\left(e^{*}, e_{*}\right)$, est un 2-isomorphisme.

1. Une erreur bénigne s'est incrustée dans [4, Rem. 2.1.161]. En effet, on y affirme qu'un morphisme fini, surjectif et totalement inséparable $e: X^{\prime} \longrightarrow X$, entre schémas de caractéristiques résiduelles nulles, induit un isomorphisme sur les schémas réduits associés. Ceci est bien entendu faux : prendre par exemple $X=\operatorname{Spec}\left(\mathbb{Q}[x, y] /\left(x^{2}-y^{3}\right)\right)$ et $X^{\prime}$ le normalisé de $X$. Cependant, on peut trouver une stratification $\mathcal{S}=\left(X_{i}\right)_{i \in I}$ de $X$, par des sous-schémas localement fermés, telle que $X_{i}^{\prime}=X^{\prime} \times_{X} X_{i} \longrightarrow X_{i}$ induit un isomorphisme sur les schémas réduits associés. En utilisant l'axiome de localité, il est alors possible de vérifier que id $\longrightarrow e_{*} e^{*}$ est inversible. D'autre part, le morphisme $e^{*} e_{*} \longrightarrow$ id est inversible par le Sous-lemme 1.4. Ceci montre que $e^{*}$ est une équivalence de catégories.

2. Bien entendu, les 2-isomorphismes $F_{n}^{*} q^{*} \simeq q^{*}, q_{*} F_{n *} \simeq q_{*}, q_{!} \simeq q_{!} F_{n !}$ et $q^{!} \simeq F_{n}^{!} q^{!}$utilisés sont ceux déduits de l'égalité $q=q \circ F_{n}$. 
(Bien entendu, $\pi_{X}$ est la projection de X $\operatorname{sur} \operatorname{Spec}(k)$.)

Demonstration On fixe un $k$-morphisme fini, surjectif et totalement inséparable $e: X^{\prime} \longrightarrow X$. Vu le Sous-lemme 1.4 ci-dessous, il reste à montrer que le 2-morphisme d'unité $\eta:$ id $\longrightarrow e_{*} e^{*}$ est inversible.

Or, le foncteur $e^{*}$ commute aux sommes infinies puisqu'il admet un adjoint à droite. Il en est de même de $e_{*}$ puisqu'il est isomorphe à $e_{\text {! }}$ (cf. [4, Th. 1.7.17]) qui possède un adjoint à droite, à savoir $e^{!}$. Puisque $\mathrm{H}$ est engendré par la base, il suffit de prouver que $\eta:$ id $\longrightarrow e_{*} e^{*}$ est inversible lorsqu'on l'applique aux objets de la forme $f_{\sharp} \pi_{Y}^{*} A$ avec $A \in \mathrm{H}(k), f: Y \longrightarrow X$ un $k$-morphisme lisse et $\pi_{Y}$ la projection de $Y$ sur $\operatorname{Spec}(k)$. Formons le carré cartésien

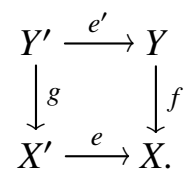

On a un diagramme commutatif (cf. [4, Prop. 1.1.9])

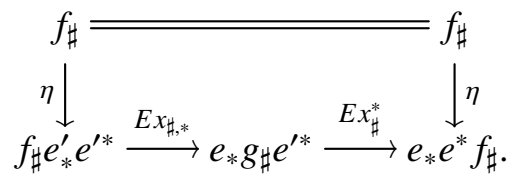

Le 2-morphisme d'échange $E x_{\sharp}^{*}: g_{\sharp} e^{\prime *} \longrightarrow e^{*} f_{\sharp}$ est inversible. Il en est de même du 2-morphisme d'échange $E x_{\sharp, *}: f_{\sharp} e_{*}^{\prime} \longrightarrow e_{*} g_{\sharp}$. En effet, modulo les égalités $f_{!}=f_{\sharp} \operatorname{Th}^{-1}\left(\Omega_{f}\right)$ et $g_{!}=g_{\sharp} \operatorname{Th}^{-1}\left(\Omega_{g}\right)$, et les 2-isomorphismes de compatibilité avec les équivalences de Thom, ce 2-morphisme d'échange correspond à $E x_{!, *}: f_{!} e_{*}^{\prime} \longrightarrow e_{*} g_{!}$. Or, modulo les 2-isomorphismes $e_{!} \simeq e_{*}$ et $e_{!}^{\prime} \simeq e_{*}^{\prime}$, le 2 morphisme d'échange $E x_{!, *}$ correspond à l'échange trivial $E x_{!, !}: f_{!} e_{!}^{\prime} \simeq e_{!} g_{!}$.

D'après la discussion précédente, on voit donc qu'il suffit de montrer que le 2-morphisme $\eta$ : $f_{\sharp} \pi_{Y}^{*} \longrightarrow f_{\sharp} e_{*}^{\prime} e^{\prime *} \pi_{Y}^{*}$ est inversible. Ceci est une conséquence de la propriété $\mathrm{P}\left(e^{\prime}\right)$.

Sous-lemme $1.4-$ Soit e $: X^{\prime} \longrightarrow X$ un k-morphisme fini, surjectif et totalement inséprable. Le 2-morphisme de counité $\delta: e^{*} e_{*} \longrightarrow$ id est inversible.

Demonstration On forme le carré cartésien à nil-immersion près :

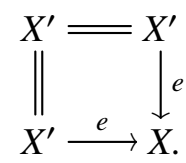

Le théorème de changement de base [4, Cor. 1.7.18] appliqué au morphisme projectif, en l'occurrence fini, $e$, fournit un 2-isomorphisme $e^{*} e_{*} \simeq \mathrm{id}_{*} \mathrm{id}^{*}$. Or, ce 2-isomorphisme est la composition de

$$
e^{*} e_{*} \stackrel{\eta}{\sim} \mathrm{id}_{*} \mathrm{id}^{*} e^{*} e_{*} \stackrel{\delta}{\longrightarrow} \mathrm{id}_{*} \mathrm{id}^{*} .
$$

Le résultat recherché s'en découle.

On a aussi besoin du fait suivant.

Lemme 1.5 - Soient e $: X^{\prime} \longrightarrow X$ et $e^{\prime}: X^{\prime \prime} \longrightarrow X^{\prime}$ des k-morphismes, finis, surjectifs et totalement inséparables.

(a) La propriété $\mathrm{P}\left(e \circ e^{\prime}\right)$ entraîne la propriété $\mathrm{P}(e)$.

(b) Les propriétés $\mathrm{P}(e)$ et $\mathrm{P}\left(e^{\prime}\right)$ entraînent la propriété $\mathrm{P}\left(e \circ e^{\prime}\right)$.

Demonstration En effet, par $\mathrm{P}\left(e \circ e^{\prime}\right)$, on a un 2-isomorphisme $\pi_{X}^{*} \simeq e_{*} e_{*}^{\prime} e^{\prime *} e^{*} \pi_{X}^{*}$. Pour montrer $\mathrm{P}(e)$, il suffit alors de vérifier que le 2-morphisme $\eta:$ id $\longrightarrow e_{*} e^{*}$ appliqué au 1-morphisme $e_{*} e_{*}^{\prime} e^{\prime *} e^{*} \pi_{X}^{*}$ est inversible. Ceci découle du Sous-lemme 1.6 ci-dessous.

Inversement, le 2-morphisme d'unité $\pi_{X}^{*} \longrightarrow\left(e \circ e^{\prime}\right)_{*}\left(e \circ e^{\prime}\right)^{*} \pi_{X}^{*}$ est la composition de

$$
\pi_{X}^{*} \longrightarrow e_{*} e^{*} \pi_{X}^{*} \longrightarrow e_{*} e_{*}^{\prime} e^{*} e^{*} \pi_{X}^{*}
$$


modulo les 2-isomorphismes évidents. La partie (b) de l'énoncé découle immédiatement de cette factorisation.

Sous-Lemme 1.6 - Soit e $: X^{\prime} \longrightarrow X$ un k-morphisme fini, surjectif et totalement inséprable. Le 2-morphisme id $\longrightarrow e_{*} e^{*}$ devient inversible lorsqu'on l'applique au 1-morphisme $e_{*}$.

Demonstration Le 2-morphisme $\eta: e_{*} \longrightarrow e_{*} e^{*} e_{*}$ admet un inverse à droite, à savoir le 2morphisme $\delta: e_{*} e^{*} e_{*} \longrightarrow e_{*}$. Or, ce dernier est inversible d'après le Sous-lemme 1.4.

Le lemme suivant s'obtient facilement en utilisant le théorème de changement de base $[\mathbf{4}$, Cor. 1.7.18] appliqué au morphisme fini $e$.

Lemme 1.7 - Soit e $: X^{\prime} \longrightarrow X$ un k-morphisme fini, surjectif et totalement inséparable. Soit $f: Y \longrightarrow X$ un $k$-morphisme et notons $e^{\prime}: X^{\prime} \times_{X} Y \longrightarrow Y$ le changement de base de e suivant $f$. Alors, la propriété $\mathrm{P}(e)$ entraîne la propriété $\mathrm{P}\left(e^{\prime}\right)$.

On établit maintenant une deuxième réduction.

Proposition 1.8 - Supposons que la propriété $\mathrm{P}\left(F_{n}\right)$ est vraie avec $F_{n}: \mathbb{A}_{k}^{d} \rightarrow \mathbb{A}_{k}^{d}$ le morphisme donné par $F_{n}\left(x_{1}, \cdots, x_{d}\right)=\left(x_{1}^{p^{n}}, \cdots, x_{d}^{p^{n}}\right)$, et cela pour tout $n, d \in \mathbb{N}$. Alors, la propriété $\mathrm{P}(-)$ est vraie pour tout morphisme fini, surjectif et totalement inséparable.

Demonstration Soit $e: X^{\prime} \longrightarrow X$ un morphisme fini, surjectif et totalement inséparable. On cherche à montrer $\mathrm{P}(e)$ sous l'hypothèse de l'énoncé. On raisonne par récurrence sur la dimension de $X$. On ne restreint pas la généralité en supposant que $X$ et $X^{\prime}$ sont réduits. Lorsque $\operatorname{dim}(X)=0$, le morphisme $e$ est inversible puisque $k$ est parfait; il n'y a donc rien à montrer.

Supposons que $\operatorname{dim}(X)>0$. Puisque $k$ est parfait et que $X$ est supposé réduit, on peut trouver un ouvert $U \subset X$ tel que $\operatorname{dim}(X-U)<\operatorname{dim}(X)$ ainsi qu'un morphisme étale $f: U \longrightarrow \mathbb{A}_{k}^{d}$ (avec $d=\operatorname{dim}(X))$. On note $Z=X-U$ et on forme le diagramme commutatif à carrés cartésiens

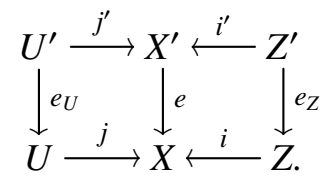

Par l'axiome de localité, pour montrer $\mathrm{P}(e)$ il suffit de montrer que

$$
j^{*} \pi_{X}^{*} \longrightarrow j^{*} e_{*} e^{*} \pi_{X}^{*} \quad \text { et } \quad i^{*} \pi_{X}^{*} \longrightarrow i^{*} e_{*} e^{*} \pi_{X}^{*}
$$

sont des 2-isomorphismes. Or, le théorème de changement de base pour un morphisme projectif (cf. [4, Cor. 1.7.18]) fournit des 2-isomorphismes

$$
j^{*} e_{*} e^{*} \simeq e_{U *} j^{*} e^{*} \simeq e_{U *} e_{U}^{*} j^{*} \quad \text { et } \quad i^{*} e_{*} e^{*} \simeq e_{Z *} i^{\prime *} e^{*} \simeq e_{Z *} e_{Z}^{*} i^{*}
$$

qui sont compatibles aux 2-morphismes d'unité des adjonctions du type image inverse et image directe. Vu que $j^{*} \pi_{X}^{*} \simeq \pi_{U}^{*}$ et $i^{*} \pi_{X}^{*} \simeq \pi_{Z}^{*}$, on est ramené à montrer $\mathrm{P}\left(e_{U}\right)$ et $\mathrm{P}\left(e_{Z}\right)$. L'hypothèse de récurrence permet de conclure quant au morphisme $e_{Z}$.

Pour montrer $\mathrm{P}\left(e_{U}\right)$, on se sert du morphisme étale $f: U \longrightarrow \mathbb{A}_{k}^{d}$. Puisque $k$ est parfait, il existe un entier $n$ suffisamment grand tel que

$$
U^{\prime} \times_{\mathbb{A}_{k}^{d}, F_{n}} \mathbb{A}_{k}^{d} \longrightarrow U \times_{\mathbb{A}_{k}^{d}, F_{n}} \mathbb{A}_{k}^{d}
$$

induit un isomorphisme sur les schémas réduits associés. En particulier, $e_{U}$ est dominé par la projection sur le premier facteur $p: U \times_{\mathbb{A}_{k}^{d}, F_{n}} \mathbb{A}_{k}^{d} \longrightarrow U$. Par le Lemme 1.5, (a), il suffit de montrer la propriété $\mathrm{P}(p)$. Or, d'après le Lemme 1.7, $\mathrm{P}(p)$ est une conséquence de $\mathrm{P}\left(F_{n}\right)$. La proposition est démontrée.

Vus le Lemme 1.3 et la Proposition 1.8, il reste à montrer la propriété $\mathrm{P}\left(F_{n}: \mathbb{A}_{k}^{d} \rightarrow \mathbb{A}_{k}^{d}\right)$ pour tout $n, d \in \mathbb{N}$ afin d'établir le Théorème 1.2. En utilisant le Lemme 1.5, (b) et le Lemme 1.7, on voit aussitôt qu'il suffit de considérer le cas $d=1$. 
Avant de continuer, nous avons besoin d'introduire une transformation naturelle $\alpha: e^{!} \longrightarrow e^{*}$, pour $e: X^{\prime} \longrightarrow X$ un morphisme fini et totalement inséparable. (Le cas qui nous intéresse est celui de $e=F_{n}$.) Pour ce faire, on utilise encore une fois le carré cartésien à nil-immersion près :

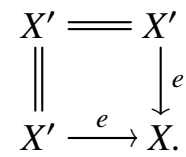

Le 2-morphisme $\alpha$ est alors la composition de

$$
e^{!} \simeq \mathrm{id}^{*} e^{!} \stackrel{E x^{\prime, *}}{\longrightarrow} \mathrm{id}^{!} e^{*} \simeq e^{*} .
$$

On note la propriété suivante.

Lemme 1.9 - On garde les notations et les hypothèses précédentes. Alors, le 2-morphisme $\alpha: e^{!} e_{*} \longrightarrow e^{*} e_{*}\left(\right.$ resp. $\left.\alpha: e^{!} e_{!} \longrightarrow e^{*} e_{!}\right)$est inversible.

Demonstration Vu le 2-isomorphisme $e_{!} \simeq e_{*}$ (cf. [4, Th. 1.7.17]), il suffit de traiter le cas non respé. Il s'agit de montrer que le 2-morphisme d'échange $E x^{!, *}: \operatorname{id}^{*} e^{!} e_{*} \longrightarrow \mathrm{id}^{!} e^{*} e_{*}$ est inversible. Par [4, Prop. 1.1.9], on dispose d'un carré commutatif

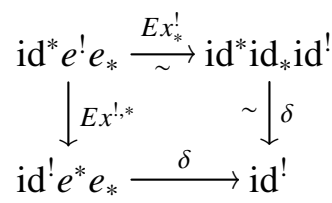

et on a déjà vu que la counité $\delta: e^{*} e_{*} \longrightarrow$ id était inversible (cf. le Sous-lemme 1.4). Ceci termine la preuve du lemme.

LEMme 1.10 - On garde les notations et les hypothèses précédentes. On a un diagramme commutatif

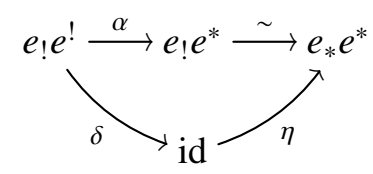

Demonstration Le 2-morphisme $e_{!} \longrightarrow e_{*}$ utilisé dans l'énoncé est celui de [4, Déf. 1.7.1]. C'est un 2-isomorphisme d'après [4, Th. 1.7.17]. Puisque $e$ est totalement inséparable, il est donné par la composition de

$$
e_{!} \simeq e_{!} \mathrm{id}_{*} \stackrel{E x_{!, *}}{\longrightarrow} e_{*} \mathrm{id}_{!} \simeq e_{*} .
$$

Ainsi, modulo les 2-isomorphismes canoniques, la composition de la ligne horizontale du diagramme (5) est égale à la composition de

$$
e_{!} \mathrm{id}_{*} \mathrm{id}^{*} e^{!} \stackrel{E x^{1, *}}{\longrightarrow} e_{!} \mathrm{id}_{*} \mathrm{id}^{!} e^{*} \stackrel{E x_{!, *}}{\longrightarrow} e_{*} \mathrm{id}_{!} \mathrm{id}^{!} e^{*} .
$$

En utilisant [4, Prop. 1.1.9], on peut former le diagramme commutatif

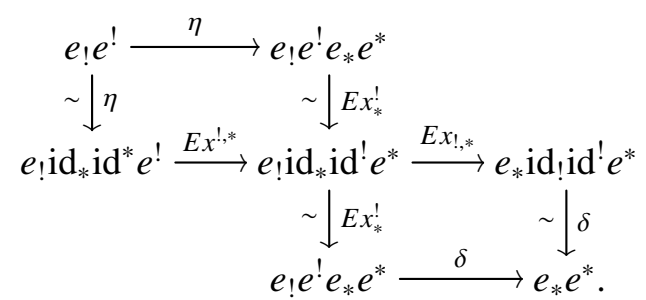

On en déduit aussitôt que la composition de la ligne horizontale du diagramme (5) est égale à la composition de

$$
e_{!} e^{!} \stackrel{\eta}{\longrightarrow} e_{!} e^{!} e_{*} e^{*} \stackrel{\delta}{\longrightarrow} e_{*} e^{*},
$$


qui est aussi la composition des flèches obliques du diagramme (5).

Proposition 1.11 - Notons $F_{n}: \mathbb{A}_{k}^{1} \longrightarrow \mathbb{A}_{k}^{1}$ le morphisme de Frobenius relatif donné par l'élévation à la puissance $p^{n}$ et $\pi$ la projection de $\mathbb{A}_{k}^{1}$ sur $\operatorname{Spec}(k)$. Supposons que la condition $\left(\mathbf{S S}_{p}\right)$ est satisfaite dans $\mathrm{H}$. Alors, le 2-morphisme $\alpha: F_{n}^{!} \pi^{*} \longrightarrow F_{n}^{*} \pi^{*}$ est inversible.

Demonstration On montrera seulement que le 2-morphisme $\alpha: F_{n}^{!} \pi^{*} \longrightarrow F_{n}^{*} \pi^{*}$ admet une section (i.e., un inverse à gauche). Ceci est suffisant pour conclure. En effet, la condition $\left(\mathbf{S S}_{p}\right)$ est autoduale. Plus précisément, elle est satisfaite dans $\mathrm{H}$ si et seulement si elle l'est dans le 2-foncteur homotopique stable $\mathrm{H}(-)^{\text {op }}$, où les foncteurs « image inverse » et « image directe » suivant un $k$-morphisme $f$ sont donnés par $\left(f^{!}\right)^{\mathrm{op}}$ et $\left(f_{!}\right)^{\mathrm{op}}$ respectivement. Ainsi, par 2-dualité, on déduit que le 2-morphisme $\alpha: F_{n}^{!} \pi^{!} \longrightarrow F_{n}^{*} \pi^{!}$admet une rétration (i.e., un inverse à droite). Il en est alors de même du 2-morphisme qui nous intéresse puisque $\pi^{!}=\pi^{*}(1)[2]$.

Clairement $F_{n}^{*} \pi^{*} \simeq \pi^{*}$. De même, on a un 2-isomorphisme $F_{n}^{!} \pi^{*} \simeq \pi^{*}$. C'est la composition de

$$
F_{n}^{!} \pi^{*} \simeq F_{n}^{!} \mathrm{Th}^{-1}\left(\Omega_{\pi}\right) \pi^{!} \simeq F_{n}^{!} \pi^{!}(-1)[-2] \simeq \pi^{!}(-1)[-2] \simeq \pi^{*} .
$$

D'autre part, l'invariance par homotopie entraîne que le 2-morphisme d'unité id $\longrightarrow \pi_{*} \pi^{*}$ est inversible. Ainsi, tout endomorphisme $\beta$ de $\pi^{*}$ est de la forme $\pi^{*} \beta_{0}$ où $\beta_{0}$ est un endomorphisme de l'identité de $\mathrm{H}(k)$. De plus, $\beta$ admet une section si et seulement si il en est ainsi de $\beta_{0}$ ou encore de $\pi_{*}(\beta)$. Pour prouver la proposition, il suffit donc de montrer que le 2-morphisme

$$
\alpha: \pi_{*} F_{n}^{!} \pi^{*} \longrightarrow \pi_{*} F_{n}^{*} \pi^{*}
$$

admet une section.

Notons $j: \mathbb{A}_{k}^{1} \hookrightarrow \mathbb{P}_{k}^{1} l^{1}$ inclusion canonique et $\infty: \operatorname{Spec}(k) \hookrightarrow \mathbb{P}_{k}^{1} l^{1}$ immersion complémentaire. Notons encore $F_{n}$ le Frobenius relatif de $\mathbb{P}_{k}^{1}$ donné par l'élévation à la puissance $p^{n}$. La projection de $\mathbb{P}_{k}^{1}$ sur $\operatorname{Spec}(k)$ sera notée $\bar{\pi}$. Le 2-morphisme

$$
\alpha: \bar{\pi}_{*} F_{n}^{!} \bar{\pi}^{*} \longrightarrow \bar{\pi}_{*} F_{n}^{*} \bar{\pi}^{*}
$$

rend le carré

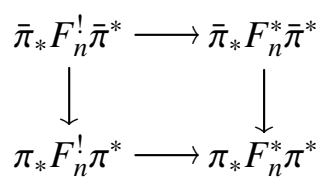

commutatif. Or, la flèche verticale de droite dans ce carré admet une section. Il suffira donc de montrer que (7) est un 2-isomorphisme.

Par le Lemme 1.10, on a un diagramme commutatif

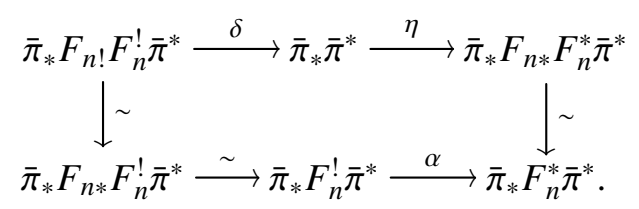

Il suffit donc de montrer que les deux 2-morphismes

$$
\eta: \bar{\pi}_{*} \bar{\pi}^{*} \longrightarrow \bar{\pi}_{*} F_{n *} F_{n}^{*} \bar{\pi}^{*} \quad \text { et } \quad \delta: \bar{\pi}_{*} F_{n !} F_{n}^{!} \bar{\pi}^{*} \longrightarrow \bar{\pi}_{*} \bar{\pi}^{*}
$$

sont des 2-isomorphismes.

Le cas de $\eta$ : On utilise le recouvrement standard de $\mathbb{P}_{k}^{1}$ par deux copies de la droite affine $\mathbb{A}_{k}^{1}$ dont l'intersection est $\mathbb{G m}_{k}$. En notant $q$ la projection de $\mathbb{G m}_{k}$ sur $\operatorname{Spec}(k)$ et $F_{n}$ le Frobenius relatif de $\mathbb{G}_{k}$ donné par l'élévation à la puissance $p^{n}$, on obtient un morphisme de 2-triangles distingués 
(dans la terminologie de [4, Déf. 1.4.5]) :

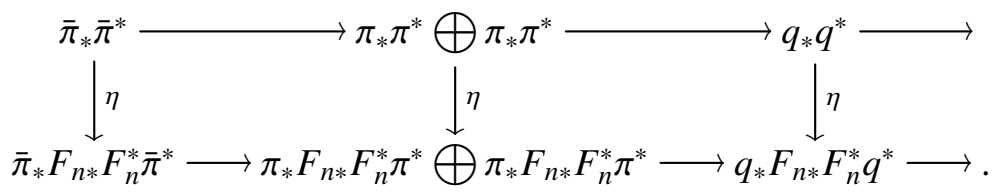

Il est facile de voir que le 2-morphisme $\eta: \pi_{*} \pi^{*} \longrightarrow \pi_{*} F_{n *} F_{n}^{*} \pi^{*}$ est inversible. On utilise pour cela le carré commutatif

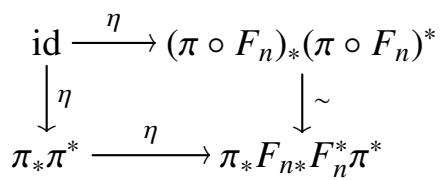

et l'invariance par homotopie. Il reste à voir que $\eta: q_{*} q^{*} \longrightarrow q_{*} F_{n *} F_{n}^{*} q^{*}$ est un 2-isomorphisme. C'est la première moitié de la condition $\left(\mathbf{S S}_{p}\right)$.

Le cas de $\delta$ : On garde les notations utilisées pour traiter le cas de $\eta$. Puisque le morphisme $\bar{\pi}$ est projectif, on a un 2-isomorphisme $\bar{\pi}_{!} \simeq \bar{\pi}_{*}$. Il suffit donc de montrer que $\delta: \bar{\pi}_{!} F_{n !} F_{n}^{!} \bar{\pi}^{*} \longrightarrow \bar{\pi}_{!} \bar{\pi}^{*}$ est inversible. Or, on dispose d'un morphisme de 2-triangles distingués :

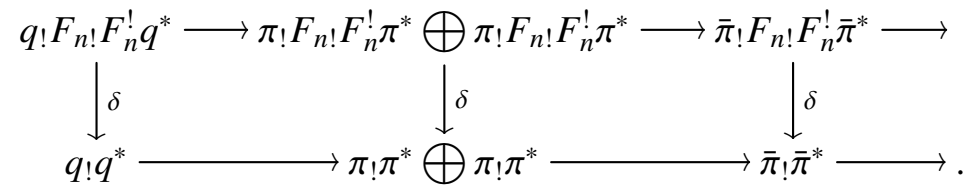

De plus, puisque $\Omega_{\pi}$ et $\Omega_{q}$ sont libres de rang 1, on a des 2-isomorphismes $\pi^{*} \simeq \pi^{!}(-1)[-2]$ et $q^{*} \simeq q^{!}(-1)[-2]$. Il suffit donc de montrer que $\delta: \pi_{!} F_{n !} F_{n}^{!} \pi^{!} \longrightarrow \pi ! \pi^{!}$et $\delta: q ! F_{n !} F_{n}^{!} q^{!} \longrightarrow q_{!} q^{!}$ sont des 2-isomorphismes. On termine comme dans le cas de $\eta$ (en utilisant la seconde moitiée de la condition $\left.\left(\mathbf{S S}_{p}\right)\right)$.

Dans la suite, on utilisera seulement la conséquence suivante de la Proposition 1.11.

Corollaire 1.12 - Gardons les hypothèses et notations de la Proposition 1.11. Le 2-morphisme $\pi^{*} \longrightarrow F_{n *} F_{n}^{*} \pi^{*}$ admet une section.

Demonstration La composition de $F_{n !} F_{n}^{!} \pi^{*} \longrightarrow \pi^{*} \longrightarrow F_{n *} F_{n}^{*} \pi^{*}$ est inversible. En effet, par le Lemme 1.10 elle coïncide avec $\alpha: F_{n *} F_{n}^{!} \pi^{*} \longrightarrow F_{n *} F_{n}^{*} \pi^{*}$ modulo le 2-isomorphisme $F_{n !} \simeq F_{n *}$. De plus, $\alpha: F_{n}^{!} \pi^{*} \longrightarrow F_{n}^{*} \pi^{*}$ est inversible par la Proposition 1.11.

Rappelons que pour terminer la preuve du Théorème 1.2, il reste à montrer que $\pi^{*} \longrightarrow F_{n *} F_{n}^{*} \pi^{*}$ est un 2-isomorphisme. On vient de voir que ce 2-morphisme admet une section. Il existe donc un projecteur $\rho$ de $\pi^{*}$ dont l'image s'identifie à $F_{n *} F_{n}^{*} \pi^{*}$, et il suffit de voir que $\rho$ est l'identité. En utilisant l'invariance par homotopie, i.e., que $\eta:$ id $\longrightarrow \pi_{*} \pi^{*}$ est inversible, on se ramène à montrer que $\pi_{*}(\rho)$ est l'identité de $\pi_{*} \pi^{*}$. Il suffit donc de montrer que $\eta: \pi_{*} \pi^{*} \longrightarrow \pi_{*} F_{n *} F_{n}^{*} \pi^{*}$ est inversible. On a déjà établi cette propriété lors de la preuve de la Proposition 1.11.

\section{Vérification de la condition $\left(\mathrm{SS}_{p}\right)$ et orientation}

L'objectif principal de ce paragraphe est de montrer que la condition $\left(\mathbf{S S}_{p}\right)$ est satisfaite dans tout 2-foncteur homotopique stable, $\mathbb{Z}[1 / 2 p]$-linéaire et séparé pour les revêtements étales d'orde 2 (cf. le Théorème 2.8 ci-dessous pour un énoncé précis). Cependant, les techniques développées servent aussi à montrer, suivant une méthode de Voevodsky [49], qu'un 2-foncteur homotopique stable $\mathbb{Q}$-linéaire et séparé est automatiquement orienté (i.e., ses équivalences de Thom sont des twists de Tate convenablement shiftés). Nous n'avons pas résisté à inclure cette application bien qu'elle ne servira pas dans la suite. 
Les résultats de ce paragraphe ne sont pas franchement nouveaux. En effet, les travaux de Morel [32] sur les groupes d'homotopie stable des sphères motiviques devraient entraîner des résultats similaires et même plus précis pour les catégories $\mathbb{A}^{1}$-homotopiques stables $\mathbf{S H}(k)$ au-dessus d'un corps $k$ (cf. l'Annexe C). En fait, notre méthode est basée sur des arguments géométriques empruntés à $[32, \S 6]$ et adaptés au langage fonctoriel qui convient pour traiter le cas général d'un 2 -foncteur homotopique stable. Aussi, pour $\mathbf{D A}{ }^{\text {ét }}(-, \Lambda)$ (cf. $\S 3$ ), la séparation est connue lorsque $\Lambda$ est une $\mathbb{Q}$-algèbre d'après Cisinski-Déglise [11]. Toutefois, pour nous il sera vital de savoir que $\mathbf{D A}^{\text {ét }}(-, \Lambda)$ est séparé pour des anneaux de coefficients plus généraux et notamment des anneaux de torsion.

Certes, le degré de généralité quasi-maximal adopté ici est une raison d'être pour ce paragraphe, mais aussi notre traitement nous semble être le plus simple et le plus direct. (Par exemple, pour l'orientation, aucune mention du cobordisme algébrique ou de la $K$-théorie n'est nécessaire.)

On fixe un schéma de base $S$ et un 2-foncteur homotopique stable $\mathrm{H}: \mathrm{Sch} / S \longrightarrow \mathfrak{I} R$. Pour le moment, on ne suppose rien sur $\mathrm{H}$. Étant donné un $S$-schéma quasi-projectif $X$, on pose $\underline{\mathrm{M}}_{c o h}(X)=$ $\pi_{X *} \pi_{X}^{*}$, où $\pi_{X}: X \longrightarrow S$ est le morphisme structural de $X$. C'est un endofoncteur de $\mathrm{H}(S)$ qui sera notre substitut au "motif cohomologique » de $X$ en l'absence d'un objet distingué dans $\mathrm{H}(S)$. Un morphisme de $S$-schémas $f: Y \longrightarrow X$ induit un 2-morphisme $\underline{\mathrm{M}}_{c o h}(X) \longrightarrow \underline{\mathrm{M}}_{c o h}(Y)$. C'est la composition de

$$
\underline{\mathrm{M}}_{c o h}(X)=\pi_{X *} \pi_{X}^{*} \stackrel{\eta}{\longrightarrow} \pi_{X *} f_{*} f^{*} \pi_{X}^{*} \simeq \pi_{Y *} \pi_{Y}^{*}=\underline{\mathrm{M}}_{c o h}(Y) .
$$

On obtient de la sorte un foncteur contravariant de $\operatorname{Sch} / S$ dans la catégorie des endofoncteurs triangulés de $\mathrm{H}(S)$. Notons le fait suivant.

Proposition 2.1 - Pour $n \in \mathbb{N}$, on a un 2-isomorphisme :

$$
\underline{\mathrm{M}}_{c o h}\left(\left(\mathbb{P}_{S}^{1}\right)^{n}\right) \simeq \bigoplus_{0 \leq i \leq n} \bigoplus_{I \subset \llbracket 1, n \rrbracket, \operatorname{card}(I)=i} \mathrm{id}(-i)[-2 i] .
$$

L'action naturelle du groupe symétrique $\Sigma_{n}$ sur $\left(\mathbb{P}_{S}^{1}\right)^{n}$ induit une action sur le foncteur $\underline{\mathrm{M}}_{c o h}\left(\left(\mathbb{P}_{S}^{1}\right)^{n}\right)$. Cette action préserve les facteurs directs

$$
\bigoplus_{I \subset \llbracket 1, n \rrbracket, \operatorname{card}(I)=i} \operatorname{id}(-i)[-2 i]
$$

pour tout $0 \leq i \leq n$. De plus, $\Sigma_{n}$ agit trivialement sur $\mathrm{id}(0)[0]$ et en permutant les facteurs directs de $\bigoplus_{1 \leq a \leq n} \mathrm{id}(-1)[-2]$.

Demonstration Cette proposition n'a rien d'étonnant. Toutefois, nous incluons une preuve par soucis d'exposition. On a id $(-i)[-2 i]=s^{!} \pi^{*}$ avec $\pi:\left(\mathbb{P}_{S}^{1}\right)^{i} \longrightarrow S$ la projection canonique et $s$ le produit des sections $[0: 1]: S \longrightarrow \mathbb{P}_{S}^{1}$. ${ }^{(3)}$ On a un 2-morphisme id(-i)[-2i] $\longrightarrow \underline{\mathrm{M}}_{c o h}\left(\left(\mathbb{P}_{S}^{1}\right)^{i}\right)$ donné par la composition de

$$
s^{!} \pi^{*} \simeq \pi_{*} s_{*} s^{\prime} \pi^{*} \stackrel{\delta}{\longrightarrow} \pi_{*} \pi^{*} .
$$

Pour $I \subset \llbracket 1, n \rrbracket$ de cardinal $i$, on définit alors un 2-morphisme $a_{I}: \operatorname{id}(-i)[-2 i] \longrightarrow \underline{\mathrm{M}}_{c o h}\left(\left(\mathbb{P}_{S}^{1}\right)^{n}\right)$ en prenant la composition de

$$
\operatorname{id}(-i)[-2 i] \longrightarrow \underline{\mathrm{M}}_{c o h}\left(\left(\mathbb{P}_{S}^{1}\right)^{i}\right) \longrightarrow \underline{\mathrm{M}}_{c o h}\left(\left(\mathbb{P}_{S}^{1}\right)^{n}\right),
$$

où la seconde flèche est induite par la projection canonique $\left(\mathbb{P}_{S}^{1}\right)^{n} \longrightarrow\left(\mathbb{P}_{S}^{1}\right)^{I}$ (modulo l'identification $\left(\mathbb{P}_{S}^{1}\right)^{I}=\left(\mathbb{P}_{S}^{1}\right)^{i}$ induite, par exemple, par l'unique bijection croissante $\left.I \simeq \llbracket 1, i \rrbracket\right)$. Nous affirmons que

$$
\sum_{I \subset \llbracket 1, n \rrbracket} a_{I}: \bigoplus_{0 \leq i \leq n} \bigoplus_{I \subset \llbracket 1, n \rrbracket, \operatorname{card}(I)=i} \mathrm{id}(-i)[-2 i] \longrightarrow \underline{\mathrm{M}}_{c o h}\left(\left(\mathbb{P}_{S}^{1}\right)^{n}\right)
$$

3. La définition originelle de id $(-i)[-2 i]$ utilise l'espace affine $\mathbb{A}_{S}^{i}$ et sa section nulle au lieu de $\left(\mathbb{P}_{S}^{1}\right)^{i}$. Toutefois, il est clair que les deux définitions sont équivalentes. 
est un 2-isomorphisme. On démontre ceci par induction sur $n$. Lorsque $n=0$ ceci est clair. On note $j: \mathbb{A}_{S}^{1} \hookrightarrow \mathbb{P}_{S}^{1}$ l'immersion complémentaire de $s: S \hookrightarrow \mathbb{P}_{S}^{1}$. On a alors un 2-triangle distingué

$$
\pi^{*} s_{*} s^{!} \pi^{*} \longrightarrow \pi_{*} \pi^{*} \longrightarrow \pi_{*} j_{*} j^{*} \pi^{*} \longrightarrow
$$

et l'unité $\eta:$ id $\longrightarrow \pi_{*} \pi^{*}$ est une section à la seconde arête de ce 2-triangle. Ceci permet de conclure pour $n=1$. Supposons maintenant que $n>0$. Le théorème de changement de base (par un morphisme lisse ou pour un morphisme projectif) appliqué au carré cartésien

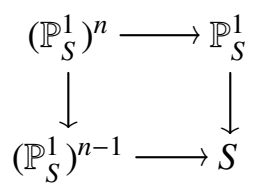

fournit un 2-isomorphisme $\underline{\mathrm{M}}_{c o h}\left(\left(\mathbb{P}_{S}^{1}\right)^{n}\right) \simeq \underline{\mathrm{M}}_{c o h}\left(\left(\mathbb{P}_{S}^{1}\right)^{n-1}\right) \circ \underline{\mathrm{M}}_{c o h}\left(\mathbb{P}_{S}^{1}\right)$. Il est facile de conclure à partir de là. (Les détails sont laissés en exercice.) Les autres assertions sont immédiates à partir de la construction du 2-morphisme (9).

Dans la suite, il sera commode d'introduire les conditions suivantes.

\section{HYPOTHÈSE 2.2}

(a) $\mathrm{H}$ est $\mathbb{Z}[1 / 2]$-linéaire (cf. [4, Déf. 2.1.153]).

(b) Le foncteur $e^{*}$ est conservatif si $e: X^{\prime} \longrightarrow X$ est un revêtement étale d'ordre 2 entre $S$ schémas quasi-projectifs.

On considère le morphisme fini surjectif

$$
f: \mathbb{P}_{S}^{1} \times_{S} \mathbb{P}_{S}^{1} \longrightarrow \mathbb{P}_{S}^{2}
$$

donné sur les coordonnées homogènes par $f([a: b],[c: d])=[a c: a d+b c: b d]$. Le morphisme $f$ identifie $\mathbb{P}_{S}^{2}$ au quotient $\left(\mathbb{P}_{S}^{1} \times_{S} \mathbb{P}_{S}^{1}\right) / \Sigma_{2}$, où $\Sigma_{2}=\{1, \tau\}$ est le groupe symétrique d'ordre 2 . Il est donc étale sur $V=\mathbb{P}_{S}^{1} \times_{S} \mathbb{P}_{S}^{1}-\Delta\left(\mathbb{P}_{S}^{1}\right)$. En fait, la restriction de $f$ à $V$ induit un revêtement étale $g: V \longrightarrow U$, où $U \subset \mathbb{P}_{S}^{2}$ est le complémentaire du sous-schéma fermé $Q$ d'équation $x_{1}^{2}-4 x_{0} x_{2}=0$. On a donc un diagramme commutatif à carrés cartésiens (à nil-immersion près) :

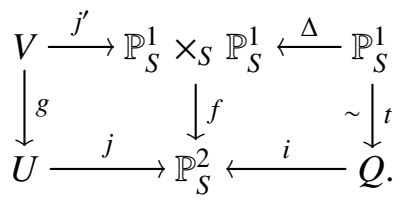

On note le lemme suivant.

Lemme 2.3 - On suppose que $\mathrm{H}$ satisfait aux conditions de l'Hypothèse 2.2. Le 2-morphisme id $\longrightarrow f_{*} f^{*}$ induit un 2-isomorphisme entre le foncteur identique de $\mathrm{H}\left(\mathbb{P}_{k}^{2}\right)$ et l'image du projecteur $\frac{1+\tau}{2}$ agissant $\operatorname{sur} f_{*} f^{*}$.

Demonstration Notons $\rho=\frac{1+\tau}{2}$ le projecteur de l'énoncé. Il s'agit de montrer que id $\longrightarrow \operatorname{Im}(\rho)$ est inversible. Par l'axiome de localité, il suffit de faire cela après application de $i^{*}$ et $j^{*}$.

Lorsqu'on applique $i^{*}$, on se ramène à identifier le foncteur identique de $\mathrm{H}(Q)$ avec l'image du projecteur $\rho$ agissant sur $t_{*} t^{*}$. Or, $t$ est un isomorphisme de sorte que le 2-morphisme d'unité id $\longrightarrow t_{*} t^{*}$ est inversible. De plus, $\tau$ agit par l'identité sur $\Delta\left(\mathbb{P}_{S}^{1}\right)$ de sorte que le projecteur $\rho$ est l'identité sur $t_{*} t^{*}$. Ceci permet de conclure dans ce cas.

Lorsqu'on applique $j^{*}$, on se ramène à identifier le foncteur identique de $\mathrm{H}(U)$ avec l'image du projecteur $\rho$ agissant sur $g_{*} g^{*}$. On obtient ceci en reprenant la la preuve de [4, Lem. 2.1.165, (2)] et en utilisant la condition (b) de l'Hypothèse 2.2 au lieu de la propriété de séparation. Pour la commodité du lecteur, nous reproduisons la preuve. Puisque $g$ est un revêtement étale d'ordre 2 , le foncteur $g^{*}$ est conservatif. On se ramène donc à montrer que $\eta: g^{*} \longrightarrow g^{*} g_{*} g^{*}$ induit un 
isomorphisme entre $g^{*}$ et l'image de $\rho$ agissant sur $g_{*} g^{*} g_{*}$. Or, le revêtement étale $g$ est trivialisé par lui-même, i.e., on a un carré cartésien

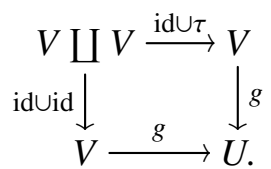

Le théorème de changement de base (par un morphisme lisse ou pour un morphisme projectif) fournit un 2-isomorphisme $\Sigma_{2}$-équivariant $g^{*} g_{*} g^{*} \simeq g^{*} \bigoplus g^{*}$, où $\tau$ agit en permutant les facteurs sur $g^{*} \bigoplus g^{*}$. Modulo cet isomorphisme, le 2-morphisme $\eta: g^{*} \longrightarrow g^{*} g_{*} g^{*}$ est donné par le morphisme diagonal $\nabla: g^{*} \longrightarrow g^{*} \bigoplus g^{*}$. Le résultat recherché est maintenant clair.

Corollaire 2.4 - On suppose que $\mathrm{H}$ satisfait aux conditions de l'Hypothèse 2.2. Alors, le 2-morphisme

$$
\underline{\mathbf{M}}_{c o h}\left(\mathbb{P}_{S}^{2}\right) \longrightarrow \underline{\mathbf{M}}_{c o h}\left(\mathbb{P}_{S}^{1} \times_{S} \mathbb{P}_{S}^{1}\right)
$$

induit un 2-isomorphisme entre $\underline{\mathrm{M}}_{c o h}\left(\mathbb{P}_{S}^{2}\right)$ et $\underline{\mathrm{M}}_{c o h}\left(\mathbb{P}_{S}^{1} \times_{S} \mathbb{P}_{S}^{1}\right)^{\Sigma_{2}}$ (i.e., l'image du projecteur $\frac{1+\tau}{2}$ agissant sur $\left.\underline{\mathrm{M}}_{\text {coh }}\left(\mathbb{P}_{S}^{1} \times_{S} \mathbb{P}_{S}^{1}\right)\right)$.

D'après la Proposition 2.1, on a

$$
\underline{\mathrm{M}}_{c o h}\left(\mathbb{P}_{S}^{1} \times_{S} \mathbb{P}_{S}^{1}\right)^{\Sigma_{2}} \simeq \mathrm{id}(0)[0] \oplus \operatorname{id}(-1)[-2] \oplus(\operatorname{id}(-2)[-4])^{\Sigma_{2}} .
$$

(D'après la Proposition 2.10 qui sera établie plus tard, $\Sigma_{2}$ agit trivialement sur id(-2)[-4].)

Corollaire 2.5 - On suppose que $\mathrm{H}$ satisfait aux conditions de l'Hypothèse 2.2. Alors, le 2morphisme $\underline{\mathrm{M}}_{\text {coh }}\left(\mathbb{P}_{S}^{2}\right) \longrightarrow \underline{\mathrm{M}}_{\text {coh }}\left(\mathbb{P}_{S}^{1}\right)$, induit par l'immersion linéaire l $: \mathbb{P}_{S}^{1} \hookrightarrow \mathbb{P}_{S}^{2}$ donnée sur les coordonnées homogènes par $l([a: b])=[0: a: b]$, admet une section.

Demonstration On a un triangle commutatif

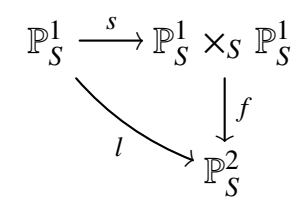

lorsque $s$ est donné par $s([a: b])=([a: b],[0: 1])$. Il est facile de voir que la composition de

$$
\underline{\mathbf{M}}_{c o h}\left(\mathbb{P}_{S}^{1} \times_{S} \mathbb{P}_{S}^{1}\right)^{\Sigma_{2}} \longrightarrow \underline{\mathbf{M}}_{c o h}\left(\mathbb{P}_{S}^{1} \times_{S} \mathbb{P}_{S}^{1}\right) \stackrel{\underline{\mathrm{M}}_{c o h}(s)}{\longrightarrow} \underline{\mathbf{M}}_{c o h}\left(\mathbb{P}_{S}^{1}\right)
$$

identifie $\underline{\mathrm{M}}_{c o h}\left(\mathbb{P}_{S}^{1}\right) \simeq \operatorname{id}(0)[0] \oplus \operatorname{id}(-1)[-2]$ avec la somme directe des deux premiers facteurs dans la décomposition (10). On conclut à l'aide du Corollaire 2.4.

La section unité de $\mathbb{G}_{S}$ induit une décomposition canonique

$$
\underline{\mathrm{M}}_{c o h}\left(\mathrm{Gm}_{S}\right) \simeq \operatorname{id} \oplus \underline{\mathrm{M}}_{c o h}\left(\mathbb{G} \mathrm{m}_{S}, 1\right) .
$$

Le recouvrement standard de $\mathbb{P}_{S}^{1}$ par deux droites affines qui s'intersectent en $\mathbb{G}_{S}$, induit un 2-triangle distingué

$$
\underline{\mathrm{M}}_{c o h}\left(\mathbb{P}_{S}^{1}\right) \longrightarrow \mathrm{id} \oplus \mathrm{id} \longrightarrow \underline{\mathrm{M}}_{c o h}\left(\mathrm{Gm}_{S}\right) \longrightarrow .
$$

On en déduit aussitôt un 2-isomorphisme $\underline{\mathrm{M}}_{c o h}\left(\mathbb{G}_{S}, 1\right) \simeq \mathrm{id}(-1)[-1]$. De même, on a une décomposition canonique

$$
\underline{\mathbf{M}}_{c o h}\left(\mathbb{G} \mathrm{m}_{S} \times_{S} \mathbb{G m}_{S}\right) \simeq \mathrm{id} \oplus \underline{\mathbf{M}}_{c o h}\left(\mathbb{G} \mathrm{m}_{S}, 1\right) \oplus \underline{\mathbf{M}}_{c o h}\left(\mathbb{G} \mathrm{m}_{S}, 1\right) \oplus \underline{\mathbf{M}}_{c o h}\left(\left(\mathbb{G} \mathrm{m}_{S}, 1\right)^{\wedge 2}\right) .
$$

Comme avant, on a un 2-isomorphisme $\underline{\mathrm{M}}_{c o h}\left(\left(\mathbb{G}_{S}, 1\right)^{\wedge 2}\right) \simeq \operatorname{id}(-2)[-2]$.

Proposition 2.6 - On suppose que $\mathrm{H}$ satisfait aux conditions de l'Hypothèse 2.2. Soit $m$ : $\mathbb{G}_{S} \times{ }_{S} \mathbb{G m}_{S} \longrightarrow \mathbb{G}_{S}$ le morphisme de multiplication. Modulo les décompositions (11) et (12), 
le 2-morphisme $\underline{\mathrm{M}}_{c o h}\left(\mathrm{Gm}_{S}\right) \longrightarrow \underline{\mathrm{M}}_{c o h}\left(\mathbb{G} \mathrm{m}_{S} \times_{S} \mathbb{G}_{S}\right)$ induit par m est donné par la matrice

$$
\left(\begin{array}{cc}
\text { id } & 0 \\
0 & \text { id } \\
0 & \text { id } \\
0 & 0
\end{array}\right) .
$$

Demonstration Toutes les entrées de la matrice sont claires sauf celle qui se trouve sur la dernière ligne et la dernière colonne. Autrement dit, il reste à montrer que la composition de

$$
\underline{\mathbf{M}}_{c o h}\left(\mathbb{G} \mathrm{m}_{S}, 1\right) \longrightarrow \underline{\mathbf{M}}_{c o h}\left(\mathbb{G} \mathrm{m}_{S}\right) \longrightarrow \underline{\mathbf{M}}_{c o h}\left(\mathbb{G} \mathrm{m}_{S} \times_{S} \mathbb{G} \mathrm{m}_{S}\right) \longrightarrow \underline{\mathbf{M}}_{c o h}\left(\left(\mathbb{G} \mathrm{m}_{S}, 1\right)^{\wedge 2}\right)
$$

est nulle. Étant donné que $x \rightsquigarrow x^{-1}$ définit un automorphisme de $\mathbb{G m}_{S}$ qui fixe la section unité, on peut remplacer le 2-morphisme au milieu dans (13) par celui induit par $d: \mathbb{G m}_{S} \times_{S} \mathbb{G}_{S} \longrightarrow \mathbb{G}_{S}$ donné par $d(x, y)=x y^{-1}$.

Suivant $[32, \S 6]$, nous considérons la fibration de Hopf $h: \mathbb{A}_{S}^{2}-0_{S} \longrightarrow \mathbb{P}_{S}^{1}$ donné par $h(x, y)=$ $[x: y]$. On dispose de deux carrés cartésiens
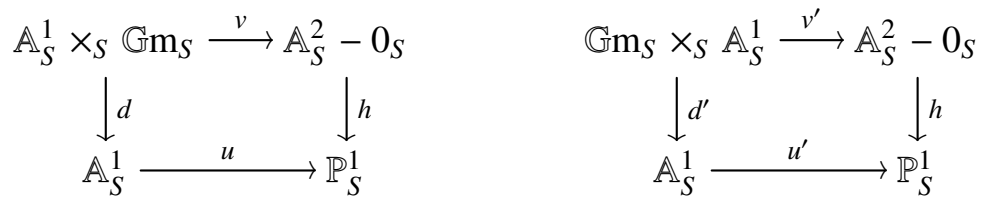

où $u(x)=[x: 1]$ et $u^{\prime}(x)=[1: x], v$ et $v^{\prime}$ sont les inclusions évidentes, et $d(x, y)=x y^{-1}$ et $d^{\prime}(x, y)=x^{-1} y$. En écrivant les triangles de Mayer-Vietoris associés aux recouvrements $\left(u, u^{\prime}\right)$ de $\mathbb{P}_{S}^{1}$ et $\left(v, v^{\prime}\right)$ de $\mathbb{A}_{S}^{2}-0_{S}$, on arrive à la conclusion que la composition de (13) coïncide, à un shift près, avec la composition de

$$
\operatorname{id}(-1)[-2] \longrightarrow \underline{\mathrm{M}}_{c o h}\left(\mathbb{P}_{k}^{1}\right) \longrightarrow \underline{\mathrm{M}}_{c o h}\left(\mathbb{A}_{S}^{2}-0_{S}\right) \longrightarrow \underline{\mathrm{M}}_{c o h}\left(\mathbb{A}_{S}^{2}-0_{S}, 1\right) \simeq \operatorname{id}(-2)[-3] .
$$

(Ci-dessus nous avons utilisé la décomposition canonique

$$
\underline{\mathbf{M}}_{c o h}\left(\mathbb{A}_{S}^{2}-0_{S}\right) \simeq \operatorname{id} \oplus \underline{\mathbf{M}}_{c o h}\left(\mathbb{A}_{S}^{2}-0_{S}, 1\right)
$$

induite par la section unité de $\mathbb{A}_{S}^{2}-0_{S}$.) Par ailleurs, le morphisme de Hopf se factorise de la manière suivante

$$
\mathbb{A}_{S}^{2}-0_{S} \stackrel{j}{\longrightarrow} \mathbb{P}_{S}^{2}-[1: 0: 0]_{S}
$$

avec $j(x, y)=[1: x: y]$ et $p([a: b: c])=[b: c]$. Il suffit donc de prouver que le 2-morphisme $\underline{\mathrm{M}}_{c o h}(j)$ composé avec la projection $\underline{\mathrm{M}}_{c o h}\left(\mathbb{A}_{S}^{2}-0_{S}\right) \longrightarrow \mathrm{id}(-2)[-3]$ est nul.

Or, $p$ est un fibré en droites de section nulle $n$ donnée par $n([x: y])=[0: x: y]$. L'invariance par homotopie entraîne que $\underline{\mathrm{M}}_{c o h}\left(\mathbb{P}_{S}^{1}\right) \longrightarrow \underline{\mathrm{M}}_{c o h}\left(\mathbb{P}_{S}^{2}-[1: 0: 0]_{S}\right)$ est un 2-isomorphisme. Ainsi, par le Corollaire 2.5, le 2-morphisme $\underline{\mathrm{M}}_{c o h}(w)$, induit par l'inclusion évidente $w: \mathbb{P}_{S}^{2}-[1: 0: 0]_{S} \hookrightarrow \mathbb{P}_{S}^{2}$, admet une section. Il suffit donc de montrer que le 2-morphisme $\underline{\mathbf{M}}_{c o h}(w \circ j)$ composé avec la projection $\underline{\mathrm{M}}_{c o h}\left(\mathbb{A}^{2}-0_{S}\right) \longrightarrow \mathrm{id}(-2)[-3]$ est nul. Mais ceci est clair puisque $w \circ j$ se factorise par $\mathbb{A}_{S}^{2}$.

Corollaire 2.7 - On suppose que $\mathrm{H}$ satisfait aux conditions de l'Hypothèse 2.2. Le 2-morphisme $\underline{\mathrm{M}}_{c o h}\left(\mathrm{Gm}_{S}\right) \longrightarrow \underline{\mathrm{M}}_{c o h}\left(\mathrm{Gm}_{S}\right)$, induit par l'élévation à la puissance n, est donné par la matrice

$$
\left(\begin{array}{ll}
1 & 0 \\
0 & n
\end{array}\right)
$$


Demonstration On raisonne par induction sur $n$; le résultat étant vrai pour $n=1$. Le morphisme $e_{n}$, d'élévation à la puissance $n$, est la composition de

$$
\mathrm{Gm}_{S} \stackrel{d_{n}}{\longrightarrow} \mathbb{G m}_{S} \times \mathbb{G m}_{S} \stackrel{m}{\longrightarrow} \mathbb{G m}_{S}
$$

avec $d(x)=\left(x^{n-1}, x\right)$. Il résulte de l'hypothèse de récurrence que le morphisme $\underline{\mathbf{M}}_{c o h}\left(d_{n}\right)$ est donné par une matrice de la forme

$$
\left(\begin{array}{cccc}
1 & 0 & 0 & 0 \\
0 & n-1 & 1 & *
\end{array}\right)
$$

dans les décompositions (11) et (12). Le résultat recherché découle maintenant de la Proposition 2.6.

On arrive maintenant au résultat principal de ce paragraphe.

Theoreme 2.8 - Soient $S$ un schéma de base et $\mathrm{H}: \mathrm{Sch} / S \longrightarrow \mathfrak{I} \Re$ un 2-foncteur homotopique stable. On suppose que les conditions suivantes sont satisfaites (avec p un nombre premier).

(a) $\mathrm{H}$ est $\mathbb{Z}[1 / 2 p]$-linéaire (cf. [4, Déf. 2.1.153]).

(b) Le foncteur $e^{*}$ est conservatif si $e: X^{\prime} \longrightarrow X$ est un revêtement étale d'ordre 2 entre $S$ schémas quasi-projectifs.

Alors, $\mathrm{H}$ satisfait à la condition $\left(\mathbf{S S}_{p}\right)$.

Demonstration Notons $F_{n}$ l'élévation à la puissance $p^{n}$ sur $\mathbb{G m}_{S /(p)}$ et $q: \mathbb{G m}_{S /(p)} \longrightarrow S /(p)$ la projection évidente. Le Corollaire 2.7, appliqué au schéma de base $S /(p)$, entraîne que $\underline{\mathrm{M}}_{c o h}\left(F_{n}\right)$ : $\underline{\mathbf{M}}_{c o h}\left(\mathbb{G m}_{S /(p)}\right) \longrightarrow \underline{\mathbf{M}}_{c o h}\left(\mathbb{G m}_{S /(p)}\right)$ est donnée par la matrice

$$
\left(\begin{array}{cc}
1 & 0 \\
0 & p^{n}
\end{array}\right)
$$

Comme $\mathrm{H}$ est supposé $\mathbb{Z}[1 / p]$-linéaire, ce 2-morphisme est inversible. En revenant à la définition du foncteur $\underline{\mathrm{M}}_{c o h}(-)$, on voit qu'on vient de prouver que la composition de

$$
q_{*} q^{*} \stackrel{\eta}{\longrightarrow} q_{*} F_{n *} F_{n}^{*} q^{*} \simeq q_{*} q^{*}
$$

est inversible. C'est la première moitié de la condition $\left(\mathbf{S S}_{p}\right)$. L'autre moitié s'en déduit par 2dualité en remarquant que les hypothèses (a) et (b) de l'énoncé sont autoduales.

Corollaire 2.9 - Gardons les hypothèses $d u$ Théorème 2.8 et supposons que $S=\operatorname{Spec}(k)$ avec $k$ un corps parfait de caractéristique $p>0$. Alors, $\mathrm{H}$ est semi-séparé.

Demonstration C'est la conjonction des Théorèmes 1.2 et 2.8 .

Notons le résultat intéressant suivant.

Propositron 2.10 - On suppose que $\mathrm{H}$ satisfait aux conditions de l'Hypothèse 2.2. Pour $n \in \mathbb{N}$, une permutation $\sigma \in \Sigma_{n}$ de $\llbracket 1, n \rrbracket$ agit sur

$$
\underline{\mathbf{M}}_{c o h}\left(\left(\mathbb{P}_{S}^{1}\right)^{n}\right) \simeq \bigoplus_{I \subset \llbracket 1, n \rrbracket} \mathrm{id}(-\operatorname{card}(I))[-2 \operatorname{card}(I)]
$$

(cf. la Proposition 2.1) en envoyant identiquement le facteur correspondant à la partie $I \subset \llbracket 1, n \rrbracket$ sur celui correspondant à la partie $\sigma^{-1}(I)$.

Demonstration On peut supposer que $\sigma$ est une transposition puisque toute permutation est un produit de transpositions. En examinant la construction du 2-isomorphisme (15) (cf. la preuve de la Proposition 2.1), on voit qu'il suffit de traiter le cas $n=2$ et, plus précisément, de montrer que l'action de $\Sigma_{2}$ sur le facteur direct id(-2)[-4] de $\underline{\mathrm{M}}_{c o h}\left(\mathbb{P}_{S}^{1} \times_{S} \mathbb{P}_{S}^{1}\right)$ est l'action triviale. Cela revient à dire que le projecteur $\frac{\mathrm{id}+\tau}{2}$ agissant sur id(-2)[-4] est inversible. Cette formulation permet d'utiliser l'axiome de localité pour distinguer le cas où 2 est inversible sur $S$ et celui où $S$ est de caractéristique 2. 
Cas où 2 est inversible sur $S$ : Considérons le carré cartésien (à nil-immersion près)

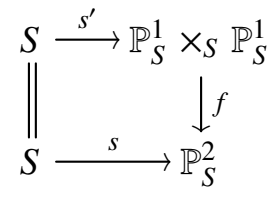

avec $s$ et $s^{\prime}$ les sections $[0: 0: 1]$ et $([0: 1],[0: 1])$. Notons $\pi: \mathbb{P}_{S}^{2} \longrightarrow S$ la projection structurale de $\mathbb{P}_{S}^{2}$. Il suffit de montrer que le 2-morphisme $s^{!} \pi^{*} \longrightarrow s^{\prime} f^{*} \pi^{*}$, donné par la composition de

$$
s^{!} \pi^{*} \simeq \mathrm{id}^{*} s^{!} \pi^{*} \stackrel{E x^{\prime, *}}{\longrightarrow} s^{\prime !} f^{*} \pi^{*},
$$

est un 2-isomorphisme. (En effet, ce 2-morphisme est $\Sigma_{2}$-équivariant et le groupe $\Sigma_{2}$ agit trivialement sur $s^{\prime} \pi^{*}$.) La question est locale au voisinage de la section $s$ pour la topologie de Zariski (et même pour la topologie de Nisnevich). Puisque 2 est inversible sur $S$, on peut remplacer le carré (16) par le carré suivant

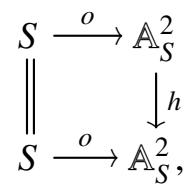

où $h(x, y)=\left(x, y^{2}\right)$ et $o$ est la section nulle. En effet, le morphisme $f: \mathbb{A}_{S}^{2} \longrightarrow \mathbb{A}_{S}^{2}$, donné par $f(x, y)=(x+y, x y)$, vérifie la relation $f \circ u=v \circ h$ avec $u$ et $v$ les automorphismes de $\mathbb{A}_{S}^{2}$ donnés par $u(x, y)=(x+y, x-y)$ et $v(a, b)=\left(2 a, a^{2}-b\right)$.

Notons $\pi: \mathbb{A}_{S}^{2} \longrightarrow S$ le morphisme structural. Nous prétendons que la composition de

$$
o ! \pi^{*} \simeq \mathrm{id}^{*} o^{!} \pi^{*} \stackrel{E x^{\prime *}}{\longrightarrow} o^{!} h^{*} \pi^{*} \simeq o^{!} \pi^{*}
$$

est la multiplication par 2. Ceci permet de conclure puisque $H$ est $\mathbb{Z}[1 / 2]$-linéaire.

L'endomorphisme de $\operatorname{id}(-2)[-4]$ donné par la composition de (18) coïncide, modulo le 2isomorphisme de composition des équivalences de Thom inverses (cf. [4, Rem. 1.5.12]), avec le 2-morphisme obtenu en appliquant id(-1)[-2] à l'endomorphisme de id(-1)[-2] induit par carré cartésien (à nil-immersion près)

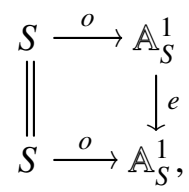

avec $e(x)=x^{2}$. Il suffit donc de montrer que la composition de

$$
o^{!} \pi^{*} \simeq \mathrm{id}^{*} o^{!} \pi^{*} \stackrel{E x^{* *}}{\longrightarrow} o^{!} e^{*} \pi^{*} \simeq o^{!} \pi^{*}
$$

est la multiplication par 2. (On a encore noté $\pi: \mathbb{A}_{S}^{1} \longrightarrow S$ la projection structurale.) Notons $j: \mathbb{G m}_{S} \hookrightarrow \mathbb{A}_{S}^{1}$ l'immersion canonique, complémentaire de la section nulle $o: S \hookrightarrow \mathbb{A}_{S}^{1}$. On a un endomorphisme du 2-triangle distingué

$$
\pi_{*} O_{*} O^{!} \pi^{*} \stackrel{\delta}{\longrightarrow} \pi_{*} \pi^{*} \stackrel{\eta}{\longrightarrow}(\pi \circ j)_{*}(\pi \circ j)^{*} \longrightarrow .
$$

Sur le premier sommet, c'est la composition de (20) modulo le 2-isomorphisme $\pi_{*} o_{*} o^{!} \pi^{*} \simeq o^{!} \pi^{*}$. Sur les deux autres sommets, il est induit par l'élévation à la puissance 2 sur $\mathbb{A}_{S}^{1}$ et $\mathbb{G m}_{S}$. Le résultat recherché découle maintenant du Corollaire 2.7.

Cas où 2 est nul sur $S:$ Notons $\tau$ la permutation des facteurs sur $\mathbb{A}_{S}^{2}, \pi: \mathbb{A}_{S}^{2} \longrightarrow S$ la projection structurale et $o: S \longrightarrow \mathbb{A}_{S}^{2}$ la section nulle. Nous allons montrer que l'action de $\tau$ sur $o^{!} \pi^{*}$ est l'identité. 
On introduit l'endomorphisme $h: \mathbb{A}_{S}^{3} \longrightarrow \mathbb{A}_{S}^{3}$ donné par $h(t, x, y)=(t, x+t(x+y), y+t(x+y))$. Puisque 2 est nul sur $S$, on a $h(0, x, y)=(0, x, y)$ et $h(1, x, y)=(1, y, x)$. Notons $\tilde{\pi}: \mathbb{A}_{S}^{3} \longrightarrow S$ la projection structurale et $\tilde{o}: \mathbb{A}_{S}^{1} \longrightarrow \mathbb{A}_{S}^{3}$ le $S$-morphisme donné par $\tilde{o}(t)=(t, 0,0)$. Alors, $h$ induit un 2-morphisme $\alpha: \tilde{o}^{!} \tilde{\pi}^{*} \longrightarrow \tilde{o}^{!} \tilde{\pi}^{*}$ donné par la composition de

$$
\tilde{o}^{!} \tilde{\pi}^{*} \simeq \mathrm{id}^{*} \tilde{o}^{!} \tilde{\pi}^{*} \stackrel{E x^{* *}}{\longrightarrow} \tilde{o}^{!} h^{*} \tilde{\pi}^{*} \simeq \tilde{o}^{!} \tilde{\pi}^{*}
$$

On note $0,1: S \longrightarrow \mathbb{A}_{S}^{1}$ la section nulle et la section unité. Modulo le 2-isomorphisme de commutation $1^{*} \tilde{o}^{!} \tilde{\pi}^{*} \simeq o^{!} \pi^{*}$ (cf. [4, Rem. 1.5.10]), le 2-morphisme $1^{*} \alpha$ est celui induit par $\tau$. Il suffit donc de montrer que $\alpha$ est l'identité. Puisque le foncteur $\tilde{o}^{!} \tilde{\pi}^{*}$ est 2 -isomorphe, aux shift et twist près, au foncteur image inverse suivant le morphisme structural $\mathbb{A}_{S}^{1} \longrightarrow S$, l'invariance par homotopie entraîne que tout endomorphisme de $\tilde{o}^{!} \tilde{\pi}^{*}$ est induit par un endomorphisme de $\mathrm{id}_{\mathrm{H}(S)}$. Par ailleurs, en utilisant le 2-isomorphisme de commutation $0^{*} \tilde{o}^{!} \tilde{\pi}^{*} \simeq o^{!} \pi^{*}$ (cf. [4, Rem. 1.5.10]), on déduit que le 2-morphisme $0^{*} \alpha$ est l'identité. Ceci entraîne que $\alpha=$ id.

Pour $n \in \mathbb{N}$, on a un $S$-morphisme $f:\left(\mathbb{P}_{S}^{1}\right)^{n} \longrightarrow \mathbb{P}_{S}^{n}$ donné par

$$
f\left(\left[x_{0}^{1}: x_{1}^{1}\right], \cdots,\left[x_{0}^{n}, x_{1}^{n}\right]\right)=\left[\prod_{s=1}^{n} x_{0}^{s}: \cdots: \prod_{s=1}^{n} x_{1}^{s}\right],
$$

où l'entrée à la $i$-ème place (avec $0 \leq i \leq n)$ est donnée par l'expression :

$$
\sum_{I \subset \llbracket 1, n \rrbracket, \operatorname{card}(I)=i} \prod_{s \in I} x_{1}^{s} \prod_{t \notin I} x_{0}^{t} .
$$

Le morphisme $f$ identifie $\mathbb{P}_{S}^{n}$ avec le quotient $\left(\mathbb{P}_{S}^{1}\right)^{n} / \Sigma_{n}$. On a le résultat suivant.

Lemme 2.11 - Gardons les notations ci-dessus. Supposons que $\mathrm{H}$ est $\mathbb{Q}$-linéaire et séparé. Alors, le 2-morphisme $\eta:$ id $\longrightarrow f_{*} f^{*}$ induit un 2-isomorphisme entre le foncteur identique de $\mathrm{H}\left(\mathbb{P}_{S}^{n}\right)$ et l'image du projecteur $\frac{1}{n !} \sum_{\sigma \in \Sigma_{n}} \sigma$ agissant sur $f_{*} f^{*}$. En particulier, le 2-morphisme $\underline{\mathrm{M}}_{c o h}\left(\mathbb{P}_{S}^{n}\right) \longrightarrow \underline{\mathrm{M}}_{c o h}\left(\left(\mathbb{P}_{S}^{1}\right)^{n}\right)^{\Sigma_{n}}$ est un 2-isomorphisme.

Demonstration La preuve est standard. Le cas $n=2$, i.e., le Lemme 2.3 contient tous les ingrédients de la preuve dans le cas général. En effet, on utilise l'axiome de localité pour se ramener au cas d'un revêtement étale galoisien et on applique alors la méthode de la preuve de $[\mathbf{4}$, Lem. 2.1.165, (2)]. Les détails sont laissés au lecteur.

Corollaire 2.12 - Supposons que $\mathrm{H}$ est $\mathbb{Q}$-linéaire et séparé. Il existe un 2-isomorphisme

$$
\underline{\mathrm{M}}_{c o h}\left(\mathbb{P}_{S}^{n}\right) \simeq \bigoplus_{0 \leq i \leq n} \mathrm{id}(-i)[-2 i]
$$

Demonstration C'est immédiat à partir de la Proposition 2.10 et du Lemme 2.11.

Theoreme 2.13 - Supposons que $\mathrm{H}$ est $\mathbb{Q}$-linéaire et séparé. Soient $\mathcal{N}$ un $\mathcal{O}_{S}$-module localement libre de rang $r+1, \mathcal{L}$ un $\mathcal{O}_{S}$-module localement libre de rang 1 et $p: \mathcal{L}^{\oplus n+1} \longrightarrow \mathcal{M}$ un morphisme surjectif de $\mathcal{O}_{S}$-modules. Notons $l: \mathbb{P}(\mathcal{N}) \hookrightarrow \mathbb{P}\left(\mathcal{L}^{\oplus n+1}\right) \simeq \mathbb{P}_{S}^{n}$ l'immersion induite par $p$. Alors, la composition de

$$
\bigoplus_{0 \leq i \leq r} \mathrm{id}(-i)[-2 i] \longrightarrow \bigoplus_{0 \leq i \leq n} \mathrm{id}(-i)[-2 i] \stackrel{(22)}{\sim} \underline{\mathrm{M}}_{c o h}\left(\mathbb{P}_{S}^{n}\right) \stackrel{\underline{\mathrm{M}}_{c o h}(l)}{\longrightarrow} \underline{\mathrm{M}}_{c o h}(\mathbb{P}(\mathcal{M}))
$$

est un 2-isomorphisme.

Demonstration La question est locale pour la topologie de Zariski sur $S$. On peut donc supposer que $\mathcal{M}=\mathcal{O}_{S}^{r+1}$ et que $\mathcal{L}=\mathcal{O}_{S}$. On raisonne par induction noethérienne sur $S$ en supposant que le théorème est vrai lorsque $S$ est remplacé par un sous-schéma fermé $T \subset S$ strict. (Le cas où $S$ est vide est trivial.) Par l'axiome de localité, il suffit donc de prouver qu'il existe un ouvert non vide $U \subset S$ tel que la composition de (23) est inversible après application de $j^{*}$, où $j: U \hookrightarrow S$ est l'inclusion. 
Or, on peut trouver un ouvert $U \subset S$ affine et non vide tel que le morphisme $p: \mathcal{O}_{U}^{n+1} \longrightarrow \mathcal{O}_{U}^{r+1}$ est le composé $p r \circ a$ avec $p r$ la projection canonique sur les premiers $r+1$ facteurs de $\mathcal{O}_{U}^{n+1}$ et $a$ un automorphisme de $\mathcal{O}_{U}^{n+1}$. Quitte à rétrécir d'avantage $U$, on peut supposer que $a=a^{\prime} \circ u_{1} \circ$ $\cdots \circ u_{m}$ avec $a$ donné par une matrice diagonale (à coefficients dans $\mathcal{O}_{S}^{\times}(U)$ ) et les $u_{i}$ unipotents. Un argument standard montre que les $\mathbb{P}\left(u_{i}\right)$ induisent l'identité sur $\underline{\mathrm{M}}_{c o h}\left(\mathbb{P}_{U}^{n}\right)$. D'autre part, $\underline{\mathrm{M}}_{c o h}\left(\mathbb{P}^{n}(a)\right)$ préserve la décomposition (22). On peut donc supposer que $a$ est l'identité. Autrement dit, on peut supposer que $l: \mathbb{P}_{S}^{r} \hookrightarrow \mathbb{P}_{S}^{n}$ est donnée par $l\left(\left[x_{0}: \cdots: x_{r}\right]\right)=\left[x_{0}: \cdots: x_{r}: 0: \cdots: 0\right]$. Dans ce cas le résultat recherché découle immédiatement de la construction du 2-isomorphisme (22). Les détails sont laissés au lecteur.

Corollaire 2.14 - Supposons que S possède un fibré inversible ample. Supposons aussi que $\mathrm{H}$ est $\mathbb{Q}$-linéaire et séparé. Alors, si $\mathcal{M}$ est un $\mathcal{O}_{S}$-module localement libre de rang $r$, il existe un 2-isomorphisme $\operatorname{Th}(\mathcal{M}) \simeq \operatorname{id}(r)[2 r]$.

Demonstration Il suffit bien entendu de construire un 2-isomorphisme $\operatorname{Th}^{-1}(\mathcal{M}) \simeq \operatorname{id}(-r)[-2 r]$. Puisque $S$ possède un fibré inversible ample, on peut trouver un $\mathcal{O}_{S}$-module $\mathcal{L}$ localement libre de rang 1 et des surjections

$$
\mathcal{L}^{\oplus n+1} \longrightarrow \mathcal{M} \quad \text { et } \quad \mathcal{L}^{\oplus m} \longrightarrow \mathcal{O}_{S} .
$$

Or, on dispose d'un 2-triangle distingué

$$
\mathrm{Th}^{-1}(\mathcal{M}) \longrightarrow \underline{\mathbf{M}}_{c o h}\left(\mathbb{P}\left(\mathcal{M} \oplus \mathcal{O}_{S}\right)\right) \longrightarrow \underline{\mathbf{M}}_{c o h}(\mathbb{P}(\mathcal{M})) \longrightarrow .
$$

D'après le Théorème 2.13, le second et le troisième sommet de ce 2-triangle sont isomorphes à $\bigoplus_{0 \leq i \leq r} \operatorname{id}(-i)[-2 i]$ et $\bigoplus_{0 \leq i \leq r-1} \operatorname{id}(-i)[-2 i]$. De plus, modulo ces identifications, la seconde arête dans ce 2-triangle est le 2-morphisme évident. Le résultat est maintenant clair.

Remarque 2.15 - Nous n'avons pas essayé de rendre le 2-isomorphisme du Corollaire 2.14 canonique. Toutefois, dans le cas qui nous intéresse le plus, à savoir celui où $H(-)=\mathbf{D A}^{\text {ét }}(-, \Lambda)$, il est possible de spécifier un tel 2-isomorphisme. On renvoie le lecteur à la Remarque 11.3 pour plus d'indications.

\section{Le 2-foncteur homotopique stable $\mathrm{DA}^{\mathrm{e}^{\mathrm{e}}(-, \Lambda)}$}

Soient $S$ un schéma et $\Lambda$ un anneau de coefficients (i.e., un anneau commutatif quelconque). On dispose d'une catégorie $\mathbf{D A}{ }^{\text {ét }}(S, \Lambda)$ du type Morel-Voevodsky. C'est la catégorie notée $\mathbb{S H H}_{\mathfrak{M}}^{T}(\mathscr{F}, \mathcal{J})$ dans [5, Déf. 4.5.21] où on prend :

- $\mathfrak{M}=\mathbf{C p l}(\Lambda)$, la catégorie des complexes de $\Lambda$-modules;

- J la catégorie à un objet et une flèche, et $\mathscr{F}$ le foncteur qui pointe $S$;

$-T=\left(\mathbb{P}_{S}^{1}, \infty_{S}\right) \otimes \Lambda$

- et $\tau=$ ét la topologie étale (cf. le début de [5, §4.5]).

Rappelons brièvement la construction de la catégorie $\mathbf{D} A^{\text {ét }}(S, \Lambda)$. Le point de départ est la catégorie $\mathrm{Sm} / S$ des $S$-schémas lisses. Sauf mention explicite du contraire, un préfaisceau sur $\mathrm{Sm} / S$ (ou ailleurs) prend ses valeurs dans la catégorie des $\Lambda$-modules. On note $\operatorname{PSh}(\operatorname{Sm} / S, \Lambda)$ la catégorie des préfaisceaux sur $\mathrm{Sm} / S$ et $\mathbf{C} \mathbf{p l}(\mathbf{P S h}(\mathrm{Sm} / S, \Lambda))$ la catégorie des complexes de préfaisceaux sur $\mathrm{Sm} / S$. Si $U$ est un $S$-schéma lisse, on a les opérations $U \otimes-$ et hom $(U,-)$ sur les complexes de préfaisceaux (cf. [5, §4.4]). Elles sont données par les formules

$$
(U \otimes K)(\dagger)=\bigoplus_{\operatorname{hom}_{S}(\dagger, U)} K(\dagger) \quad \text { et } \quad \underline{\operatorname{hom}}(U, K)(\dagger)=K\left(U \times_{S} \dagger\right)
$$

où † est un $S$-schéma lisse et $K$ un complexe de préfaisceaux sur $\operatorname{Sm} / S$. En fait, hom $(U,-)$ est l'adjoint à droite du foncteur $U \otimes-$.

La catégorie $\mathbf{C p l}(\mathbf{P S h}(\operatorname{Sm} / S, \Lambda))$ possède une structure de modèles projective où les équivalences faibles sont les quasi-isomorphismes et les fibrations sont les surjections. Une localisation de Bousfield de la structure projective fournit la structure projective ét-locale sur cette catégorie 
(cf. [5, Déf. 4.4.33]). Les équivalences ét-locales sont les morphismes de complexes de préfaisceaux sur $\mathrm{Sm} / S$ induisant des isomorphismes sur les faisceaux étales associés aux préfaisceaux d'homologie. Le foncteur de faisceautisation induit alors une équivalence de catégories triangulées

$$
a_{\text {ét }}: \mathbf{H o}_{\text {ét }}(\mathbf{C p l}(\mathbf{P S h}(\operatorname{Sm} / S, \Lambda))) \stackrel{\sim}{\longrightarrow} \mathbf{D}\left(\mathbf{S h}_{\text {ét }}(\operatorname{Sm} / S, \Lambda)\right)
$$

entre la catégorie homotopique de la structure ét-locale et la catégorie dérivée de la catégorie abélienne $\mathbf{S h}_{\text {ét }}(\mathrm{Sm} / S, \Lambda)$ des faisceaux étales sur $\mathrm{Sm} / S$.

On localise d'avantage la structure projective ét-locale suivant la classe des morphismes $\mathbb{A}_{X}^{1} \otimes$ $\Lambda[n] \longrightarrow X \otimes \Lambda[n]$ avec $X \in \mathrm{Sm} / S$ et $n \in \mathbb{Z}$. On obtient ainsi la structure de modèles ( $\mathbb{A}^{1}$, ét)-locale $\operatorname{sur} \mathbf{C p l}(\mathbf{P S h}(\operatorname{Sm} / S, \Lambda))$ (cf. [5, Déf. 4.5.12]). On note

$$
\mathbf{D A}^{\text {eff, ét }}(S, \Lambda)=\mathbf{H o}_{\mathbb{A}^{1} \text {-ét }}(\mathbf{C p l}(\mathbf{P S h}(\operatorname{Sm} / S, \Lambda))) \text {, }
$$

sa catégorie homotopique. C'est la catégorie triangulée des $S$-motifs étales effectifs (version sans transferts) à coefficients dans $\Lambda$. Le $S$-motif effectif étale associé à un $S$-schéma lisse $X$ est simplement le préfaisceau $X \otimes \Lambda$ vu comme un objet de $\mathbf{D A}^{\text {eff,ét }}(S, \Lambda)$.

La dernière étape consiste à stabiliser la construction précédente. Notons $T_{S}$, ou simplement $T$ s'il n'y a pas de confusion possible, le préfaisceau quotient $\left(\mathbb{P}_{S}^{1}, \infty_{S}\right) \otimes \Lambda=\left(\mathbb{P}_{S}^{1} \otimes \Lambda\right) /\left(\infty_{S} \otimes \Lambda\right)$. On considère la catégorie $\operatorname{Spt}_{T}^{\Sigma}(\mathbf{C p l}(\mathbf{P S h}(\operatorname{Sm} / S, \Lambda)))$ des $T$-spectres symétriques en complexes de préfaisceaux sur $\mathrm{Sm} / S$ (cf. [5, Déf. 4.3.6 et 4.5.18]). On munit cette catégorie de la structure de modèles stable déduite de la structure projective ( $\mathbb{A}^{1}$, ét)-locale (cf. [5, Déf. 4.3.29]). C'est la structure projective $\left(\mathbb{A}^{1}\right.$, ét)-locale stable. On note

$$
\mathbf{D A}^{\text {ét }}(S, \Lambda)=\mathbf{H o}_{\mathbb{A}^{1}-e ́ t-s t}\left(\operatorname{Spt}_{T}^{\Sigma}(\mathbf{C p l}(\mathbf{P S h}(\operatorname{Sm} / S, \Lambda)))\right),
$$

sa catégorie homotopique. C'est la catégorie triangulée des $S$-motifs étales (version sans transferts) à coefficients dans $\Lambda$. Le $S$-motif étale associé à un $S$-schéma lisse $X$ est simplement le $T$-spectre symetrique $\operatorname{Sus}_{T, \Sigma}^{0}(X \otimes \Lambda)$ vu comme un objet de $\mathbf{D A}^{\text {ét }}(S, \Lambda)$. (Rappelons que le $p$-ième foncteur de suspension infinie $\operatorname{Sus}_{T, \Sigma}^{p}$ est l'adjoint à gauche du foncteur $\operatorname{Ev}_{p}$ qui envoie un $T$-spectre symétrique $\mathbf{E}=(\mathbf{E})_{n \in \mathbb{N}}$ sur le complexe de préfaisceaux $\mathbf{E}_{p}$. Pour plus de détails, nous renvoyons le lecteur à [5, Déf. 4.3.10].) Voici quelques notations standards qu'on utilisera couramment.

\section{Notation 3.1}

(i) Les catégories $\mathbf{D} \mathbf{A}^{\text {eff, ét }}(S, \Lambda)$ et $\mathbf{D} \mathbf{A}^{\text {ét }}(S, \Lambda)$ sont monoïdales, symétriques, unitaires et fermées. Leur produit tensoriel sera noté $-\otimes_{\Lambda}-$ ou simplement $-\otimes-$ lorsqu'il n'y a pas de confusion possible sur l'anneau de coefficients. Le bifoncteur « homomorphisme interne » est généralement noté $\underline{\operatorname{Hom}}(-,-)$.

(ii) On notera $\Lambda_{S}(0)$ l'objet de $\mathbf{D A} A^{\text {ét }}(S, \Lambda)$ donné par $\operatorname{Sus}_{T, \Sigma}^{0}\left(\Lambda_{c s t}\right)$ avec $\Lambda_{c s t}$ le préfaisceau constant de valeur $\Lambda$ sur $\mathrm{Sm} / S$. Autrement dit, $\Lambda_{S}(0)$ est le motif étale de $S \in \mathrm{Sm} / S$ et c'est l'objet unité pour la structure monoïdale sur $\mathbf{D A}^{\text {ét }}(S, \Lambda)$. On pose aussi $\Lambda_{S}(1)=\operatorname{Sus}_{T, \Sigma}^{0}\left(T_{S}[-2]\right)$. C'est un objet inversible pour le produit tensoriel de $\mathbf{D} A^{\text {ét }}(S, \Lambda)$ et son inverse est $\Lambda_{S}(-1)=$ $\operatorname{Sus}_{T, \Sigma}^{1}\left(\Lambda_{c s t}[2]\right)$. Ceci permet de définir les motifs de Tate $\Lambda_{S}(n)$ pour tout $n \in \mathbb{Z}$. Lorsqu'il n'y a pas de confusion possible, nous omettrons la mention de la base et nous noterons simplement $\Lambda(n)$ les motifs de Tate.

(iii) Enfin, si $M \in \mathbf{D A}{ }^{\text {ét }}(S, \Lambda)$ est un motif étale, les twist de Tate de $M$ sont définis par $M \otimes \Lambda(n)$ pour tout $n \in \mathbb{Z}$; on les notera aussi $M(n)$.

Pour les constructions complètes et de nombreux compléments, le lecteur est prié de consulter [5, Chap. 4] où l'on considère une situation nettement plus générale. Notons cependant la proposition suivante dont la partie essentielle, à savoir l'axiome de localité, est due à Morel et Voevodsky [37], même si ces derniers considèrent le contexte non linéaire, instable et local pour la topologie Nisnevich de la théorie $\mathbb{A}^{1}$-homopique des schémas.

Proposition 3.2 - Le 2-foncteur $\mathbf{D A}{ }^{\text {ét }}(-, \Lambda)$, restreint à $\mathrm{Sch} / S$, est un 2-foncteur homotopique stable au sens de [4, Déf. 1.4.1]. 
Demonstration C'est la conjonction de [5, Cor. 4.5.47] et des résultats de [5, §4.5.4].

Les résultats de [4] s'appliquent donc au 2-foncteur $\mathbf{D A}^{\text {ét }}(-, \Lambda): \operatorname{Sch} / S \longrightarrow \mathfrak{I R}$ pour tout schéma de base $S$.

Remarque 3.3 - Dans la construction des catégories $\mathbf{D A}{ }^{\text {eff, ét }}(S, \Lambda)$ et $\mathbf{D} A^{\text {ét }}(S, \Lambda)$ on peut utiliser la topologie Nisnevich au lieu de la topologie étale. On obtient alors les variantes Nisnevich $\mathbf{D A}^{\text {eff }}(S, \Lambda)$ et $\mathbf{D A}(S, \Lambda)$ de ces catégories. Le 2-foncteur $\mathbf{D A}(-, \Lambda): \operatorname{Sch} / S \longrightarrow \mathfrak{I} \Re$ est lui aussi un 2-foncteur homotopique stable.

Le lemme suivant renforce [4, Prop. 1.4.3]. Il est propre à la variante étale.

Lemme 3.4 - Soit $\left(u_{i}: U_{i} \longrightarrow S\right)_{i}$ un recouvrement étale d'un schéma $S$. Alors, les foncteurs $u_{i}^{*}: \mathbf{D A}^{\mathrm{ét}}(S, \Lambda) \longrightarrow \mathbf{D A}^{\mathrm{ét}}\left(U_{i}, \Lambda\right)$ forment une famille conservative.

Demonstration Soit $\mathbf{E}$ un $T$-spectre symétrique en complexes de préfaisceaux sur $\mathrm{Sm} / S$. Supposons que $\mathrm{L} u_{i}^{*}(\mathbf{E})$ est nul dans $\mathbf{D A}^{\text {ét }}\left(U_{i}, \Lambda\right)$ pour tout indice $i$. On cherche à montrer que $\mathbf{E}$ est nul dans $\mathbf{D A}^{\text {ét }}(S, \Lambda)$. On ne restreint pas la généralité en supposant que $\mathbf{E}$ est projectivement stablement $\left(\mathbb{A}^{1}\right.$, ét)-fibrant. Le foncteur

$$
u_{i}^{*}: \operatorname{Spt}_{T}^{\Sigma}(\operatorname{Cpl}(\operatorname{PSh}(\operatorname{Sm} / S, \Lambda))) \longrightarrow \operatorname{Spt}_{T}^{\Sigma}\left(\operatorname{Cpl}\left(\operatorname{PSh}\left(\operatorname{Sm} / U_{i}, \Lambda\right)\right)\right)
$$

est de Quillen à droite puisque $u_{i}$ est lisse (cf. [5, Th. 4.5.23]). On en déduit aussitôt que $u_{i}^{*} \mathbf{E}$ est encore projectivement stablement $\left(\mathbb{A}^{1}\right.$, ét)-fibrant. En particulier, les complexes de préfaisceaux $u_{i}^{*} \mathbf{E}_{n}$ sont acycliques pour tout $n \in \mathbb{N}$. Puisque la famille $\left(u_{i}: U_{i} \longrightarrow S\right)_{i}$ est un recouvrement étale, on déduit aussitôt que les complexes de faisceaux aét $\left(\mathbf{E}_{n}\right)$ sont acycliques. Il s'ensuit que $\mathbf{E}$ est nul dans $\mathbf{D A}^{\text {ét }}(S, \Lambda)$.

D'après [4, Cor. 1.7.18], le théorème de changement de base pour un morphisme projectif est valable dans $\mathbf{D A}^{\text {ét }}(-, \Lambda)$ pour les carrés cartésiens de $S$-schémas quasi-projectifs. En fait, il est aussi valable pour les changements de base généraux.

Proposition 3.5 - Supposons donné un carré cartésien de schémas

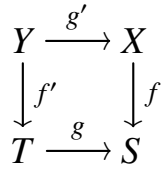

avec $f$ projectif. (On ne suppose pas que g est de type fini!) Alors, le 2-morphisme

$$
E x_{*}^{*}: g^{*} f_{*} \longrightarrow f_{*}^{\prime} g^{*}
$$

est un 2-isomorphisme entre foncteurs de $\mathbf{D} \mathbf{A}^{\text {ét }}(X, \Lambda)$ dans $\mathbf{D} \mathbf{A}^{\text {ét }}(T, \Lambda)$.

Demonstration Il s'agit de vérifier que la preuve de [4, Cor. 1.7.18], ainsi que les preuves des résultats intermédiaires sur lesquels elle repose, gardent un sens lorsque $g$ n'est pas nécessairement quasi-projectif. Pour le lecteur qui souhaite entreprendre cette vérification, nous reprenons les grandes lignes de la preuve en indiquant les points essentiels.

En factorisant $f$ par une immersion fermée suivie de la projection d'un espace projectif relatif, on se ramène à traiter le cas où $f$ est une immersion fermée et celui où le morphisme $f$ est supposé lisse.

Cas d'une immersion fermée $i$ : Il suffit de remarquer que l'argument de [4, Lem. 1.4.14] ne fait usage d'aucune hypothèse sur le morphisme $g$.

Cas où $f$ est projectif et lisse : On utilisera l'opération $f !=f_{\sharp} \circ \mathrm{Th}^{-1}\left(\Omega_{f}\right)$ ainsi que le 2-morphisme $f_{!} \longrightarrow f_{*}$ qui est inversible puisque $f$ est projectif. La commutation avec les équivalences de Thom, établie dans [4, Prop. 1.5.2] pour les foncteurs « image inverse » suivant des morphismes quasiprojectifs, s'étend aux foncteurs « image inverse » suivant des morphismes des schémas arbitraires. (On utilise pour cela le cas d'une immersion fermée, considéré ci-dessus, ainsi que le Lemme 3.6 ci-dessous.) On a donc un 2-isomorphisme canonique

$$
g^{\prime *} \mathrm{Th}^{-1}\left(\Omega_{f}\right) \simeq \mathrm{Th}^{-1}\left(\Omega_{f^{\prime}}\right) g^{\prime *} .
$$


Ceci permet de définir un 2-morphisme $E x_{!}^{*}: f_{!}^{\prime} g^{\prime *} \longrightarrow g^{*} f_{!}$comme dans [4, Prop. 1.5.19 (4)] qui est un 2-isomorphisme par le Lemme 3.6 ci-dessous. La preuve de [4, Prop. 1.7.7] s'étend sans modification au cas où $g$ n'est plus supposé quasi-projectif pour fournir un diagramme commutatif

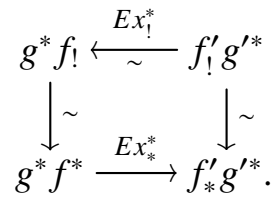

(Cette preuve repose en fait sur la commutation de l'isomorphisme de pureté avec les foncteurs « image inverse » (cf. [4, Cor. 1.6.23]) qui s'étend, elle aussi, au cas d'un changement de base non nécessairement quasi-projectif.) Ceci termine la démonstration de la proposition.

LEMme 3.6 - Supposons donné un carré cartésien de schémas

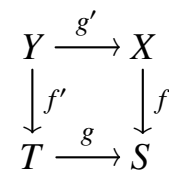

avec $f$ lisse. (On ne suppose pas que g est de type fini!) Alors, le 2-morphisme

$$
E x_{\sharp}^{*}: f_{\sharp}^{\prime} g^{\prime *} \longrightarrow g^{*} f_{\sharp}
$$

est un 2-isomorphisme dans $\mathbf{D} \mathbf{A}^{\mathrm{e}}{ }^{\mathrm{et}}(-, \Lambda)$.

Demonstration Il suffit de remarquer que l'argument de [5, Prop. 4.5.48] ne fait usage d'aucune hypothèse sur le morphisme $g$.

On aura également besoin du résultat suivant.

Proposition 3.7 - Pour tout schéma $S$, la catégorie $\mathbf{D A}{ }^{\mathrm{e} t}(S, \Lambda)$ coüncide avec sa plus petite souscatégorie triangulée stable par sommes infinies et contenant les objets de la forme $\operatorname{Sus}_{T \Sigma}^{n}(X \otimes \Lambda)$ avec $n \in \mathbb{N}$ et $X \in \mathrm{Sm} / S$.

Demonstration On note provisoirement $\mathcal{T} \subset \mathbf{D A}^{\text {ét }}(S, \Lambda)$ la plus petite sous-catégorie triangulée stable par sommes infinies et contenant les objets de la forme $\operatorname{Sus}_{T, \Sigma}^{n}(X \otimes \Lambda)$ avec $n \in \mathbb{N}$ et $X \in$ $\mathrm{Sm} / S$. Soit $\mathbf{E}$ un $T$-spectre symétrique en complexes de préfaisceaux sur $\mathrm{Sm} / S$ et supposons que $\mathbf{E}$ est projectivement cofibrant. Alors, le morphisme $0 \longrightarrow \mathbf{E}$ est un rétract d'un morphisme de la forme $0 \longrightarrow \mathbf{F}$ appartenant à

$$
\operatorname{Cell}\left(\left\{\operatorname{Sus}_{T, \Sigma}^{n}(c \otimes X) ; n \in \mathbb{N}, X \in \operatorname{Sm} / S, c \in \operatorname{Cof}_{p r o j}(\operatorname{Cpp}(\Lambda))\right\}\right) .
$$

(Pour la signification de Cell(-), nous renvoyons le lecteur à [5, Déf. 4.2.24].) Autrement dit, $\mathbf{E}$ est un facteur direct de la colimite d'un système inductif $\left(\mathbf{F}_{\mu}\right)_{\mu \in \nu}$ indéxé par un ordinal limite $v$ et vérifiant les conditions suivantes :

(a) $\mathbf{F}_{\emptyset}=0$.

(b) $\mathbf{F}_{\mu} \longrightarrow \mathbf{F}_{\mu+1}$ est un «push-out » d'un coproduit de morphismes de la forme $\operatorname{Sus}_{T, \Sigma}^{n}(c \otimes X)$ avec $n \in \mathbb{N}, X \in \mathrm{Sm} / S$ et $c$ une cofibration projective de complexes de $\Lambda$-modules.

(c) $\mathbf{F}_{\mu}=\operatorname{colim}_{\rho \in \mu} \mathbf{F}_{\rho}$ si $\mu \in v$ est un ordinal limite.

Une induction transfinie permet alors de montrer que $\mathbf{F}_{\mu}$ appartient à $\mathcal{T}$ pour tout $\mu \in v+1$. Le passage de $\mu$ à $\mu+1$ découle immédiatement de (b). Le passage à un ordinal limite, utilise le fait qu'une colimite homotopique d'un diagramme ayant ses sommets dans $\mathcal{T}$ est un objet de $\mathcal{T}$. (La preuve de cette propriété est standard ; elle est laissée au lecteur.)

Le résultat suivant est une conséquence immédiate de la Proposition 3.7.

Corollaire 3.8 - Soit $S$ un schéma de base. Le 2-foncteur homotopique stable $\mathbf{D A}{ }^{\text {ét }}(-, \Lambda)$ : $\mathrm{Sch} / S \longrightarrow$ IR est engendré par la base au sens de [4, Déf. 2.1.155].

On arrive maintenant au résultat clef suivant. 
Theoreme 3.9 - Soient $S$ un schéma de base et $\Lambda$ un anneau. Alors, le 2-foncteur homotopique stable $\mathbf{D} A^{\text {ét }}(-, \Lambda): \mathrm{Sch} / S \longrightarrow \mathfrak{I R}$ est séparé.

Demonstration Par le Lemme 3.4, joint à [4, Prop. 2.1.162], il reste à montrer que le 2-foncteur homotopique stable $\mathbf{D A}{ }^{\text {ét }}(-, \Lambda): \operatorname{Sch} / S \longrightarrow \mathfrak{I} \mathfrak{R}$ est semi-séparé. On fixe un morphisme $e$ : $S^{\prime} \longrightarrow S$ fini, surjectif et totalement inséparable. Vu le Sous-lemme 1.4, qui est encore valable sans que $e$ soit un morphisme quasi-projectif sur un corps, il suffit de montrer que le 2-morphisme $\eta:$ id $\longrightarrow e_{*} e^{*}$ est un 2-isomorphisme. Soit $U \subset S$ le plus grand ouvert Zariski tel que $\kappa(x) \simeq$ $\kappa\left(e^{-1}(x)\right)$ pour tout $x \in U$. (Bien entendu, $\kappa(-)$ désigne le corps résiduel d'un point.) Puisque $S$ est noethérien, on peut écrire $U$ comme l'union d'un nombre fini de sous-schémas localement fermés $T_{1}, \ldots, T_{r} \subset U$ tels que $T_{i} \times_{S} S^{\prime} \longrightarrow T_{i}$ induit un isomorphisme sur les schémas réduits associés. L'axiome de localité et le théorème de changement de base pour un morphisme fini entraînent alors que $\eta:$ id $\longrightarrow e_{*} e^{*}$ est inversible pour $e: e^{-1}(U) \longrightarrow U$ et qu'il reste à montrer que ce 2morphisme est inversible pour $e: S^{\prime}-e^{-1}(U) \longrightarrow S-U$. Or, par maximalité de $U$, le schéma $S-U$ est de caractéristique non nulle. Quitte à remplacer $S$ par une composante connexe de $S-U$, on peut donc supposer depuis le départ que $S$ est de caractéristique $p>0$. D'après le Lemme 3.10 ci-dessus, $\mathbf{D A}^{\mathrm{ét}}(-, \Lambda)$ est alors $\mathbb{Z}[1 / p]$-linéaire.

On ne restreint pas la généralité en supposant que $S$ est affine. Les foncteurs $e^{*}$ et $e_{*}$, étant des adjoints à gauche de $e_{*}$ et $e^{!}$, ils commutent aux sommes infinies. Par la Proposition 3.7, il suffit donc de prouver que $\eta$ : id $\longrightarrow e_{*} e^{*}$ est inversible après évaluation sur des objets de la forme $\operatorname{Sus}_{T, \Sigma}^{n}(X \otimes \Lambda)$, avec $n \in \mathbb{N}$ et $X \in \mathrm{Sm} / S$. Or, on peut trouver un $\mathbb{F}_{p}$-schéma affine de type fini $S_{0}$, un morphisme de schémas $g: S \longrightarrow S_{0}$ et un schéma lisse $X_{0} \in \mathrm{Sm} / S_{0}$ tels que $X \simeq X_{0} \times_{S_{0}} S$. Il est alors suffisant de prouver que $\eta: g^{*} \longrightarrow e_{*} e^{*} g^{*}$ est un 2-isomorphisme. Quitte à raffiner $S_{0}$, on peut aussi supposer que $e$ est le changement de base d'un morphisme fini, surjectif et totalement inséparable $e_{0}: S_{0}^{\prime} \longrightarrow S_{0}$. En notant $g^{\prime}: S^{\prime} \longrightarrow S_{0}^{\prime}$ le morphisme canonique, on a alors les 2-isomorphismes

$$
g^{*} e_{0 *} e_{0}^{*} \simeq e_{*} g^{*} e_{0}^{*} \simeq e_{*} e^{*} g^{*}
$$

(Pour le premier 2-isomorphisme, on utilise la Proposition 3.5 appliquée au morphisme fini $e_{0}$.) Il est donc suffisant de prouver que $\eta:$ id $\longrightarrow e_{0 *} e_{0}^{*}$ est un 2-isomorphisme. Autrement dit, on peut supposer que $S$ est $\mathbb{F}_{p}$-schéma quasi-projectif. Dans ce cas, on est en mesure d'appliquer le Théorème 1.2. On est alors ramené à vérifier la propriété $\left(\mathbf{S S}_{p}\right)$ pour $\mathbf{D A}{ }^{\mathrm{e}}(-, \Lambda): \operatorname{Sch} / \mathbb{F}_{p} \longrightarrow \mathfrak{I} \Re$. Cette propriété est bien satisfaite par le Théorème 2.8 joint au Lemme 3.4 (si $\Lambda$ est une $\mathbb{Z}[1 / 2]$ algèbre ou $p=2$ ) ou par le Théorème C.1 (sans hypothèse sur $\Lambda$ ni $p$ ).

Lemme 3.10 - Soient $S$ un $\mathbb{F}_{p}$-schéma et $\Lambda$ un anneau. Alors, $\mathbf{D A}^{\text {ét }}(S, \Lambda)$ est $\mathbb{Z}[1 / p]$-linéaire.

Demonstration Il est suffisant de montrer que le morphisme $p \times-: \operatorname{Sus}_{T}^{0}\left(\Lambda_{c s t}\right) \longrightarrow \operatorname{Sus}_{T}^{0}\left(\Lambda_{c s t}\right)$ est inversible dans $\mathbf{D A}{ }^{\text {ét }}(S, \Lambda)$. (En effet, $\mathbf{D A}^{{ }^{\text {ét }}}(S, \Lambda)$ est une catégorie monoïdale et $\operatorname{Sus}_{T}^{0}\left(\Lambda_{c s t}\right)$ est son objet unité.) Il est donc suffisant de montrer que le morphisme $p \times-: \Lambda_{c s t} \longrightarrow \Lambda_{c s t}$ est inversible dans $\mathbf{D A}{ }^{\text {eff, ét }}(S, \Lambda)$. Il est même suffisant de montrer que le faisceau étale constant $\mathbb{Z} / p \mathbb{Z}$ est isomorphe au faisceau nul dans $\mathbf{D A}^{\text {eff, ét }}\left(\mathbb{F}_{p}, \mathbb{Z}\right)$. Or, on dispose d'une suite exacte de faisceaux étales sur $\mathrm{Sm} / \mathbb{F}_{p}$ :

$$
0 \longrightarrow \mathbb{Z} / p \mathbb{Z} \longrightarrow \mathcal{O} \stackrel{x \mapsto x^{p}-x}{\longrightarrow} \mathcal{O} \longrightarrow 0
$$

et le faisceau $\mathcal{O}$ est bien $\mathbb{A}^{1}$-équivalent au faisceau nul.

En utilisant le Corollaire 2.14, on déduit le résultat suivant.

Corollaire 3.11 - Soit $S$ un schéma de base et supposons que $\Lambda$ est une $\mathbb{Q}$-algèbre. Alors, le 2 -foncteur homotopique stable $\mathbf{D A}^{\mathrm{et}}(-, \Lambda): \mathrm{Sch} / S \longrightarrow \mathfrak{T} \mathfrak{R}$ est orienté. Autrement dit, pour tout $\mathcal{O}_{S}$-module $\mathcal{M}$ localement libre de rang $r$, il existe un 2-isomorphisme $\operatorname{Th}(\mathcal{M}) \simeq \mathrm{id}(r)[2 r]$.

Le reste de ce paragraphe est consacré à quelques compléments techniques sur les catégories $\mathbf{D A}^{\text {ét }}(S, \Lambda)$. On rappelle d'abord des notions de dimension cohomologique suivant [3, Expo. X] et $[44, \S 3]$.

Definition 3.12 - Soit p un nombre premier. 
1. La p-dimension cohomologique $\mathrm{cd}_{p}(k)$ d'un corps $k$ est le supremum (dans $\mathbb{N} \cup\{+\infty\}$ ) de l'ensemble des entiers $n$ pour lesquels le groupe abélien $\mathrm{H}^{n}\left(\mathrm{Gal}\left(k^{\text {sep }} / k\right), M\right)$ est non nul pour au moins une $\mathrm{Gal}\left(k^{\text {sep }} / k\right)$-représentation $M$ de p-torsion. (Bien entendu, $k^{\text {sep }}$ est une clôture séparable de $k$ et $\mathrm{Gal}\left(k^{\text {sep }} / k\right)$ est son groupe de Galois. $)$

2. La p-dimension cohomologique ponctuelle $\operatorname{pcd}_{p}(S)$ d'un schéma $S$ est le supremum (dans $\mathbb{N} \cup\{+\infty\}$ ) des nombres $\mathrm{cd}_{p}(\kappa(s))$ quand s parcourt les points de $S$. (Bien entendu, $\kappa(s)$ est le corps résiduel du point $s \in S$.)

Remarque 3.13 - Soient $k$ est un corps de nombre et $p$ un nombre premier. Si $p$ est impair, on $\operatorname{a~cd}_{p}(k)=2$ par un théorème de Poitou et Tate. Ceci reste vrai pour $p=2$ lorsque $k$ n'admet pas de plongements réels. (Voir [44, Chap. II, §4.4, Prop. 13].)

Les deux lemmes suivants sont bien connus.

Lemme 3.14 - Si $\operatorname{pcd}_{p}(S)$ est finie, il en est de même de $\operatorname{pcd}_{p}(T)$ pour tout $S$-schéma de type fini $T$.

Demonstration Il s'agit de [44, §4, Prop. 11].

Lemme 3.15 - Soient $S$ un schéma et $\mathcal{F}$ un faisceau de p-torsion sur le petit site étale de $S$. Alors $\mathrm{H}_{\text {ét }}^{n}(S, \mathcal{F})=0$ si $n \geq\left(1+\operatorname{pcd}_{p}(S)\right)(1+\operatorname{krdim}(S))$. ${ }^{(4)}$

Demonstration Ceci découle de [3, Expo. X, Th. 4.1 et 5.1] joint à [44, §4, Prop. 10].

Du même tonneau, on a le résultat suivant.

Lemme 3.16 - Soient $S$ un schéma et $\mathcal{F}$ un faisceau de $\mathbb{Q}$-espaces vectoriels sur le petit site étale de $S$. Alors $\mathrm{H}_{\mathrm{et}}^{n}(S, \mathcal{F})=0$ si $n \geq \operatorname{krdim}(S)$.

Demonstration On utilise l'isomorphisme $\mathrm{H}_{\dot{e} \mathrm{t}}^{\cdot}(S, \mathcal{F}) \simeq \mathrm{H}_{\mathrm{Nis}}^{\cdot}(S, \mathcal{F})$, vrai pour tout faisceau $\mathcal{F}$ de Q-espaces vectoriels sur le petit site étale de $S$, et le fait que $\mathrm{H}_{\mathrm{Nis}}^{n}(S, \mathcal{F})=0$ pour $n>\operatorname{krdim}(S)$ (cf. [48]).

Pour un schéma de base $S$ et un anneau de coefficients $\Lambda$, nous introduisons l'hypothèse suivante.

Нypothèse 3.17 - La dimension de Krull de $S$ est finie et les $p$-dimensions cohomologiques ponctuelles de $S$ sont uniformément bornées lorsque $p$ parcourt les nombres premiers non inversibles dans $\Lambda .{ }^{(5)}$

Lemme 3.18 - Soient $S$ un schéma et $\Lambda$ un anneau vérifiant l'Hypothèse 3.17. Alors, les deux propriétés suivantes sont satisfaites :

(a) les colimites filtrantes préservent les objets projectivement ét-fibrants de $\mathbf{C p l}(\mathbf{P S h}(\mathrm{Et} / S, \Lambda))$;

(b) pour tout $U \in \mathrm{Et} / S$, le foncteur $\mathrm{H}_{\mathrm{et}}^{0}(U,-): \mathbf{C p l}(\mathbf{P S h}(\mathrm{Et} / S, \Lambda)) \longrightarrow \operatorname{Mod}(\Lambda)$ commute aux colimites filtrantes.

Demonstration L'implication (b) $\Rightarrow$ (a) est standard. (L'argument est contenu dans la preuve de [5, Prop. 4.5.62].) La propriété (b) se démontre de la même manière que [5, Cor. 4.5.61]. On doit uniquement faire attention au fait que le petit site étale $\mathrm{Et} / S$ n'est pas nécéssairement de dimension cohomologique finie au sens de [5, Déf. 4.5.57]. Cepandant, les Lemmes 3.15 et 3.16 entraînent que les foncteurs $\mathrm{H}_{\mathrm{et}}^{i}(U,-)$, pour $U \in \mathrm{Et} / S$, restreints aux faisceaux de $\Lambda$-modules, sont tous nuls pour $i$ suffisamment grand. Il en découle que les conclusions de [5, Prop. 4.5.58, Cor. 4.5.60 et Cor. 4.5.61] restent valables pour $\mathcal{S}=\mathrm{Et} / S$ et $\mathfrak{M}=\mathbf{C p l}(\Lambda)$.

Proposition 3.19 - Soient $S$ un schéma et $\Lambda$ un anneau satisfaisant à l'Hypothèse 3.17. Alors, la catégorie triangulée $\mathbf{D} \mathbf{A}^{\mathrm{e} t}(S, \Lambda)$ est compactement engendrée par les objets de la forme $\operatorname{Sus}_{T, \Sigma}^{n}(X \otimes$ $\Lambda)$ où $n \in \mathbb{N}$ et $X \in \mathrm{Sm} / S$.

4. Nous ne prétendons pas de donner une borne optimale.

5. Pour la suite, on peut se contenter de demander que les $p$-dimensions cohomologiques ponctuelles soient finies individuellement. Toutefois, l'existence d'une borne finie commune permet de simplifier les arguments et c'est souvent satisfait en pratique. 
Demonstration La preuve de cette proposition est essentiellement la même que celle de [5, Th. 4.5.67] qui traite le cas de la topologie Nisnevich. On utilise le Lemme 3.18 pour obtenir l'analogue de [5, Prop. 4.5.62] pour le site $\mathrm{Sm} / S$, muni de la topologie étale, et la catégorie de modèles $\mathfrak{M}=\mathbf{C p l}(\Lambda)$. Les preuves de [5, Prop. 4.5.63, Cor. 4.5.64] se transportent alors sans difficulté au cas de $\mathbf{C p l}(\mathbf{P S h}(\operatorname{Sm} / S, \Lambda))$ munie de la structure de modèles projective ( $\mathbb{A}^{1}$, ét)-locale (au lieu de $\left(\mathbb{A}^{1}\right.$, Nis)-locale comme dans loc. cit. $)$. Ceci permet de reprendre la preuve de [5, Th. 4.5.67] dans la situation qui nous intéresse.

Voici un autre énoncé connu pour les catégories $\mathbf{D A}(S, \Lambda)$ (cf. [6, Prop. 1.A.1]) et que nous étendons au cas de $\mathbf{D} \mathbf{A}^{\text {ét }}(S, \Lambda)$.

Proposition 3.20 - Soient I une petite catégorie cofiltrante et $\left(X_{i}\right)_{i \in I}$ un pro-schéma. On suppose que les morphismes de transition $f_{j \rightarrow i}: X_{j} \longrightarrow X_{i}$ sont affines de sorte que la limite projective $X=$ $\lim _{i \in I} X_{i}$ existe dans la catégorie des schémas. On suppose aussi que $X$ et les $X_{i}$ sont noethériens et qu'ils satisfont à l'Hypothèse 3.17 relativement à un anneau de coefficients $\Lambda$.

Pour $i \in I$, notons $f_{\infty \rightarrow i}: X \longrightarrow X_{i}$ le morphisme canonique. Soient $i_{0} \in I$, et $M$ et $N$ deux objets de $\mathbf{D} \mathbf{A}^{\mathrm{et}}\left(X_{i_{0}}, \Lambda\right)$. Si M est compact, alors l'homomorphisme

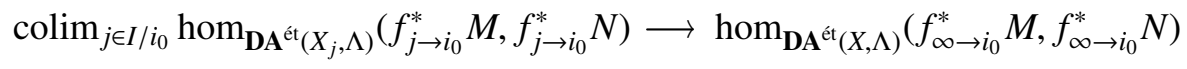

est bijectif. L'énoncé analogue vaut aussi pour les catégories effectives $\mathbf{D A}^{\mathrm{eff}, \mathrm{e} t}(-, \Lambda)$.

Demonstration La preuve suit la même stratégie que celle de [6, Prop. 1.A.1]. Pour la commodité du lecteur, nous reprenons les arguments.

On traite uniquement le cas stable. On peut supposer que $i_{0} \in I$ est l'objet final, qu'on notera $o$. On peut aussi supposer que $M=\operatorname{Sus}_{T, \Sigma}^{n}\left(Y_{o} \otimes \Lambda\right)$ avec $n \in \mathbb{N}$ et $Y_{o} \in \operatorname{Sm} / X_{o}$. On choisit un $T_{X_{o}}$-spectre symétrique projectivement cofibrant $\mathbf{N}=\left(\mathbf{N}_{n}\right)_{n \in \mathbb{N}}$ qui représente $N$.

On peut considérer le pro-schéma $\left(X_{i}\right)_{i \in I}$ comme un diagramme de schémas $(\tilde{X}, I)$ en posant $\tilde{X}(i)=X_{i}$. On note $\pi:(\tilde{X}, I) \longrightarrow X_{o}$ la projection évidente et on considère le $T_{\tilde{X}}$-spectre symétrique $\pi^{*} \mathbf{N}$, un objet de $\operatorname{Spt}_{T}^{\Sigma}\left(\mathbf{C p l}(\mathbf{P S h}(\operatorname{Sm} /(\tilde{X}, I), \Lambda))\right.$ ). Pour $i \in I$, on a $i^{*}\left(\pi^{*} \mathbf{N}\right)=f_{i \rightarrow 0}^{*} \mathbf{N}$ (où l'on note par $i$ le morphisme de diagrammes de schémas $i:\left(X_{i}, \mathbf{e}\right) \longrightarrow(\tilde{X}, I)$ qui est l'identité sur $\left.X_{i}\right)$. On choisit ensuite une cofibration projective stablement ( $\mathbb{A}^{1}$, ét)-triviale $\pi^{*} \mathbf{N} \longrightarrow \mathbf{R}$ avec $\mathbf{R}$ un $T_{\tilde{X}}$-spectre symétrique stablement ( $\mathbb{A}^{1}$, ét)-fibrant. Pour tout $i \in I$, le morphisme évident $f_{i \rightarrow 0}^{*} \mathbf{N} \longrightarrow i^{*} \mathbf{R}$ est une cofibration stablement ( $\mathbb{A}^{1}$, ét)-triviale et $i^{*} \mathbf{R}$ est stablement ( $\mathbb{A}^{1}$, ét)-fibrant. En particulier, on a des isomorphismes canoniques

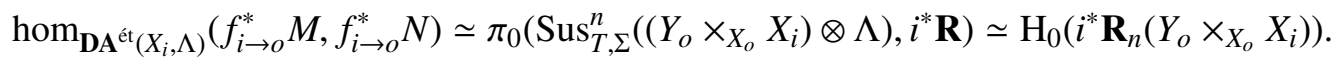

Par ailleurs, pour une flèche $j \rightarrow i$ dans $I$, on a un morphisme de $T_{X_{j}}$-spectres symétriques $f_{j \rightarrow i}^{*} i^{*} \mathbf{R} \longrightarrow j^{*} \mathbf{R}$. C'est une équivalence $\left(\mathbb{A}^{1}\right.$, ét)-locale stable comme il découle immédiatement de la propriété 2 de 3 appliquée à la suite

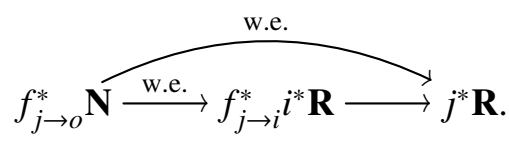

De plus, on dispose d'un carré commutatif

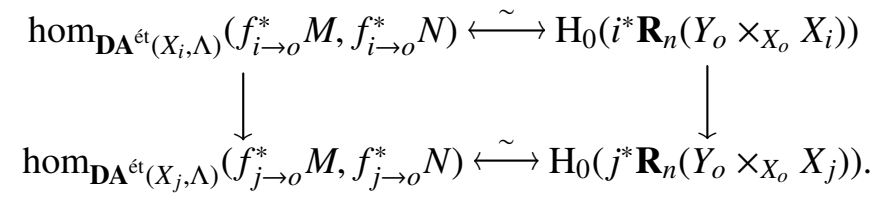

Considérons à présent le $T_{X}$-spectre symétrique $\hat{\mathbf{R}}=\operatorname{colim}_{i \in I} f_{\infty \rightarrow i}^{*}{ }^{*} \mathbf{R}$. On dispose d'un morphisme évident $f_{\infty \rightarrow 0}^{*} \mathbf{N} \longrightarrow \hat{\mathbf{R}}$ qui est une équivalence ( $\mathbb{A}^{1}$, ét)-locale stable. En effet, les morphismes $f_{\infty \rightarrow 0}^{*} \mathbf{N} \longrightarrow f_{\infty \rightarrow i}^{*} i^{*} \mathbf{R}$ sont des cofibrations stablement ( $\mathbb{A}^{1}$, ét)-triviales (étant donné que 
les $f_{\infty \rightarrow i}^{*}$ sont des foncteurs de Quillen à gauche) et le foncteur

$$
\operatorname{colim}_{I}: \operatorname{Spt}_{T}^{\Sigma}(\mathbf{C p l}(\mathbf{P S h}(\operatorname{Sm} /(X, I), \Lambda))) \longrightarrow \operatorname{Spt}_{T}^{\Sigma}(\mathbf{C p l}(\mathbf{P S h}(\operatorname{Sm} / X, \Lambda)))
$$

préserve les équivalences $\left(\mathbb{A}^{1}\right.$, ét)-locales stables (étant donné qu'il est de Quillen à gauche et qu'il préserve les quasi-isomorphismes de complexes de préfaisceaux niveau par niveau).

Nous allons voir maintenant que le $T_{X}$-spectre symétrique $\hat{\mathbf{R}}$ est stablement ( $\mathbb{A}^{1}$, ét)-fibrant. Soient $n \in \mathbb{N}$ et $Y \in \mathrm{Sm} / X$. On peut trouver $i \in I$ et $Y_{i} \in \mathrm{Sm} / X_{i}$ tels que $Y \simeq Y_{i} \times_{X_{i}} X$ (cf. [6, Lem. 1.A.2]). Il est facile de voir que

$$
\hat{\mathbf{R}}_{n}(Y)=\operatorname{colim}_{j \in I / i} j^{*} \mathbf{R}_{n}\left(Y_{j \rightarrow i}\right)
$$

avec $Y_{j \rightarrow i}=Y_{i} \times_{X_{i}} X_{j}$. À l'aide de cette formule et du Lemme 3.21 ci-dessus, on déduit que $\hat{\mathbf{R}}_{n}$ est ét-fibrant pour tout $n$. La formule (26) montre aussi que le morphisme $\hat{\mathbf{R}}_{n}(Y) \longrightarrow \hat{\mathbf{R}}_{n}\left(\mathbb{A}_{Y}^{1}\right)$ est un quasi-isomorphisme, l'analogue de ceci étant vrai pour les complexes $j^{*} \mathbf{R}_{n}$. Autrement dit, $\hat{\mathbf{R}}$ est projectivement $\left(\mathbb{A}^{1}\right.$, ét)-local niveau par niveau. Il reste à voir que c'est un $\Omega$-spectre, i.e., que $\hat{\mathbf{R}}_{n}(Y) \longrightarrow \hat{\mathbf{R}}_{n+1}\left(\mathbb{P}_{Y}^{1}, \infty_{Y}\right)$ est un quasi-isomorphisme. Ceci est encore une conséquence immédiate de la formule (26) et du fait que les $j^{*} \mathbf{R}$ sont des $\Omega$-spectres.

À présent, il est facile de conclure. En effet, par la discussion précédente, on a

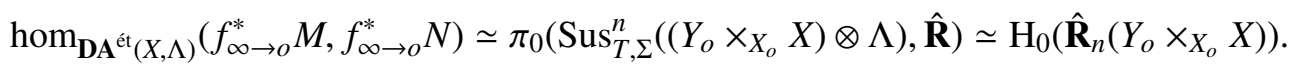

Mais par (26), on a

$$
\mathrm{H}_{0}\left(\hat{\mathbf{R}}_{n}\left(Y_{o} \times_{X_{o}} X\right)\right) \simeq \operatorname{colim}_{i \in I} \mathrm{H}_{0}\left(i^{*} \mathbf{R}_{n}\left(Y_{o} \times_{X_{o}} X_{i}\right)\right) .
$$

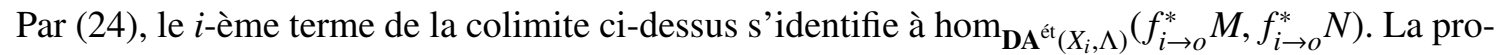
position est démontrée.

Lemme 3.21 - On garde les hypothèses et les notations de la Proposition 3.20 concernant le pro-schéma $\left(X_{i}\right)_{i \in I}$. On note $(\tilde{X}, I)$ le diagramme de schémas donné par $\tilde{X}(i)=X_{i}$. Soit $K$ un complexe de préfaisceaux de $\Lambda$-modules sur $\mathrm{Et} /(\tilde{X}, I)$. Alors, l'homomorphisme évident

$$
\operatorname{colim}_{i \in I} \mathrm{H}_{\dot{e ̂ t}}^{\cdot}\left(X_{i}, i^{*} K\right) \longrightarrow \mathrm{H}_{\text {êt }}^{\cdot}\left(X, \operatorname{colim}_{i \in I} f_{\infty \rightarrow i}^{*} i^{*} K\right) \text {. }
$$

est bijectif. De plus, si les complexes de préfaisceaux $i^{*} K$ sont projectivement ét-fibrants pour tout $i \in I$, il en est de même de $\operatorname{colim}_{i \in I} f_{\infty \rightarrow i}^{*} i^{*} K$.

Demonstration On prouve d'abord que (27) est bijectif. Lorsque $K$ est un préfaisceau (placé en degré zéro), il s'agit de [2, Expo. VII, Th. 5.7]. Une récurrence facile permet de traiter le cas où $K$ est borné. Pour le cas général, on écrit $K=\operatorname{colim}_{n \in \mathbb{N}} K^{(n)}$ avec $K^{(n)}=\tau_{\geq-n}\left(\sigma_{\leq n}(K)\right.$ ), une troncation bête suivie d'une troncation canonique de $K$. Par le Lemme 3.18, les homomorphismes évidents

$$
\begin{gathered}
\operatorname{colim}_{n \in \mathbb{N}} \mathrm{H}_{\text {êt }}^{\cdot}\left(X_{i}, i^{*} K^{(n)}\right) \longrightarrow \mathrm{H}_{\text {ét }}^{*}\left(X_{i}, i^{*} K\right) \quad \text { pour } i \in I \\
\text { et } \quad \operatorname{colim}_{n \in \mathbb{N}} \mathrm{H}_{\text {êt }}^{\cdot}\left(X, \operatorname{colim}_{i \in I} f_{\infty \rightarrow i}^{*} i^{*} K^{(n)}\right) \longrightarrow \mathrm{H}_{\text {ét }}^{\cdot}\left(X, \operatorname{colim}_{i \in I} f_{\infty \rightarrow i}^{*} i^{*} K\right)
\end{gathered}
$$

sont des bijections. Ceci démontre la première assertion de l'énoncé.

La seconde assertion s'en déduit par la méthode standard. Soit $u: \operatorname{colim}_{i \in I} f_{\infty \rightarrow i}^{*} i^{*} K \longrightarrow L$ une équivalence ét-locale avec $L$ un complexe de préfaisceaux ét-fibrant sur $E t / X$. Nous allons montrer que $u$ est un quasi-isomorphisme de complexes de préfaisceaux, ce qui permettra de conclure. Soit $U \in \mathrm{Et} / X$. On peut trouver $i \in I$ et $U_{i} \in \mathrm{Et} / X_{i}$ tels que $U \simeq X \times_{X_{i}} U_{i}$. En utilisant que (27) est bijectif pour le pro-schéma $\left(U_{j \rightarrow i}=X_{j} \times_{X_{i}} U_{i}\right)_{j \rightarrow i \in I / i}$ et la restriction de $K$ à $(\tilde{U}, I / i)$, on obtient que l'homomorphisme

$$
\operatorname{colim}_{j \rightarrow i \in I / i} \mathrm{H}_{\mathrm{et}}^{\cdot}\left(X_{j} \times_{X_{i}} U_{i}, j^{*} K\right) \longrightarrow \mathrm{H}_{\mathrm{et}}^{\cdot}(U, L)
$$

est bijectif. Puisque $j^{*} K$ et $L$ sont supposés ét-fibrants, on déduit que

$$
\operatorname{colim}_{j \rightarrow i \in I / i} K\left(X_{j} \times_{X_{i}} U_{i}\right) \longrightarrow L(U)
$$

est un quasi-isomorphisme. Or, le membre de gauche s'identifie à $\Gamma\left(U, \operatorname{colim}_{j \in I} f_{\infty \rightarrow j}^{*} j^{*} K\right)$. Ceci termine la preuve du lemme. 
L'énoncé ci-dessus se déduit de la Proposition 3.20 de la même manière que [6, Cor. 1.A.3] se déduit de [6, Prop. 1.A.1].

Corollaire 3.22 - Gardons les hypothèses et les notations de la Proposition 3.20. La souscatégorie pleine $\mathbf{D} \mathbf{A}_{\mathrm{ct}}^{\mathrm{et}}(X, \Lambda)$ des objets compacts de $\mathbf{D} \mathbf{A}^{\mathrm{et}}(X, \Lambda)$ est équivalente à la 2-colimite des sous-catégories $\mathbf{D A}_{\mathrm{ct}}^{\mathrm{et}}\left(X_{i}, \Lambda\right)$ des objets compacts de $\mathbf{D} \mathbf{A}^{\mathrm{t}}\left(X_{i}, \Lambda\right)$. L'énoncé analogue vaut aussi pour les catégories effectives $\mathbf{D} \mathbf{A}^{\mathrm{eff}}$ ét $(-, \Lambda)$.

Remarque 3.23 — L'analogue du Corollaire 3.22 pour les catégories $\mathbf{H o}_{\text {ét }}(\mathbf{C p l}(\mathbf{P S h}(\mathrm{Et} /-, \Lambda)))$ est également vrai. On peut l'obtenir à l'aide du Lemme 3.21. On laisse les détails au lecteur.

On termine avec un dernier résultat bien utile en pratique.

Proposition 3.24 - Soient $S$ un schéma et $\Lambda$ un anneau satisfaisant à l'Hypothèse 3.17. Alors, les foncteurs $x^{*}: \mathbf{D A}^{\mathrm{e}}{ }^{\mathrm{t}}(S, \Lambda) \longrightarrow \mathbf{D A}^{\mathrm{e}}{ }^{\mathrm{t}}(x, \Lambda)$, pour $x \in S$, forment une famille conservative.

Demonstration On divise la preuve en trois étapes.

Étape $A$ : Étant donné un point $x \in S$, on note $x^{!}: \mathbf{D A}^{\text {ét }}(S, \Lambda) \longrightarrow \mathbf{D A}^{\text {ét }}(x, \Lambda)$ le foncteur composé $g_{x}^{*} \circ t_{x}^{!}$où $t_{x}: \overline{\{x\}} \hookrightarrow S$ est l'immersion fermée évidente et $g_{x}: x \hookrightarrow \overline{\{x\}}$ l'inclusion du point générique de $\overline{\{x\}}$. On prouve ici que la famille $\left(x^{!}\right)_{x \in S}$ est conservative. Pour cela, on raisonne par induction noethérienne en supposant que le résultat est prouvé pour tout sous-schéma fermé strict de $S$. (Lorsque $S$ est vide, il n'y a rien à prouver!)

Soit $P \in \mathbf{D A}^{\text {ét }}(S, \Lambda)$ tel que $x ! P=0$ pour tout $x \in S$. On cherche à montrer que $P=0$. Puisque DA $^{\text {ét }}(S, \Lambda)$ est compactement engendrée (voir la Proposition 3.19), il suffit de prouver que toute flèche $\alpha: M \longrightarrow P$, partante d'un objet compact $M \in \mathbf{D A}^{\text {ét }}(S, \Lambda)$, est nulle.

Soit $\eta$ un point générique de $S$. Puisque $\eta^{*}(\alpha)=\eta^{!}(\alpha)=0$, la Proposition 3.20 montre qu'il existe un voisinage ouvert $U \subset S$ de $\eta$ tel que $j^{*}(\alpha)=0$ avec $j: U \hookrightarrow S$ l'inclusion évidente. Autrement dit, la composition de $j_{\sharp} j^{*} M \longrightarrow M \longrightarrow P$ est nulle. Il vient que $\alpha$ se factorise par le morphisme d'unité $M \longrightarrow i_{*} i^{*} M$ avec $i: Z \hookrightarrow S$ l'immersion fermée complémentaire à $j$. Il suffit donc de prouver que toute flèche de la forme $\beta: i_{*} i^{*} M \longrightarrow P$ est nulle. Par adjonction, cette flèche correspond à $\beta^{\prime}: i^{*} M \longrightarrow i^{!} P$. Or, pour tout $z \in Z$, on a $z^{!}\left(i^{!} P\right) \simeq(i(z)) ! P=0$. L'hypothèse d'induction entraîne que $i ! P=0$. Ceci prouve le résultat recherché.

Étape $B$ : Pour tout $x \in S$, on note $j_{x}: \operatorname{Spec}\left(\mathcal{O}_{S, s}\right) \longrightarrow S$ le morphisme évident. Considérons le carré cartésien

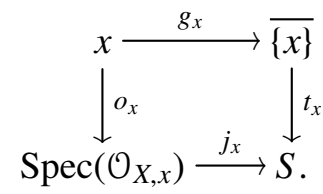

L'isomorphisme de changement de base $j_{x}^{*} t_{x *} \simeq o_{x *} g_{x}^{*}$ (cas facile de la Proposition 3.5) induit une transformation naturelle $E x^{* !}: g_{x}^{*} t_{x}^{!} \longrightarrow o_{x}^{!} j_{x}^{*}$. Il est facile de voir qu'elle est inversible. Pour cela, on peut utiliser la Proposition 3.20 et raisonner comme dans la preuve de [6, Cor. 1.A.4]. Vue l'étape A, on déduit aussitôt que la famille $\left(j_{x}^{*}\right)_{x \in S}$ est conservative.

Étape $C$ : On peut maintenant démontrer que la famille $\left(x^{*}\right)_{x \in S}$ est conservative. D'après l'étape B, on peut supposer que $S$ est local et donc en particulier de dimension de Krull fini.

Dans la suite, on raisonne par récurrence sur la dimension de Krull de $S$. Lorsque cette dimension est nulle, $S$ possède un nombre fini de points qui sont tous ouverts dans $S$. Le résultat est alors clair.

Supposons que $\operatorname{krdim}(S)>1$. Par l'étape B, on peut supposer que $S$ est local de point fermé $s$. Notons $j: U=S-\{s\} \hookrightarrow S$ l'inclusion de l'ouvert complémentaire à $s$. Soit $P \in \mathbf{D A}^{\text {ét }}(S, \Lambda)$ tel que $x^{*} P=0$ pour tout $x \in S$. Alors, $j^{*} P$ est tel que $u^{*}\left(j^{*} P\right)=0$ pour tout $u \in U$. Comme $\operatorname{krdim}(U)<\operatorname{krdim}(X)$, on peut utiliser l'hypothèse de récurrence pour déduire que $j^{*} P=0$. D'autre part, on a par hypothèse $s^{*} P=0$. On conclut que $P=0$ par l'axiome de localité. 


\section{Le théorème de rigidité relatif}

Soit $S$ un schéma de base. Rappelons que Et/S désigne la catégorie des $S$-schémas étales. On munit $\operatorname{Cpl}(\mathbf{P S h}(E t / S, \Lambda))$ de la structure projective ét-locale dont les équivalences faibles sont les morphismes de complexes de préfaisceaux induisant des isomorphismes sur les faisceaux étales associés aux préfaisceaux d'homologie. La catégorie homotopique de cette structure sera notée $\mathbf{D}^{\text {ét }}(S, \Lambda)$. Elle est équivalente à $\mathbf{D}\left(\mathbf{S h}_{e ̂ t}(\mathrm{Et} / S, \Lambda)\right)$, la catégorie dérivée de la catégorie abélienne des faisceaux étales de $\Lambda$-modules sur $S$.

L'inclusion évidente $\iota_{S}: \mathrm{Et} / S \hookrightarrow \mathrm{Sm} / S$ est un morphisme de sites. En effet, la catégorie Et $/ S$ possède les limites finies et le foncteur $\iota_{S}$ y commute (cf. [1, Expo. IV, Prop. 4.9.4]). On en déduit aussitôt que le foncteur

$$
\iota_{S}^{*}: \mathbf{C p l}(\mathbf{P S h}(\mathrm{Et} / S, \Lambda)) \longrightarrow \mathbf{C p l}(\mathbf{P S h}(\operatorname{Sm} / S, \Lambda))
$$

préserve les équivalences ét-locales. Comme il préserve aussi les cofibrations projectives, il est de Quillen à gauche. En composant avec $\operatorname{Sus}_{T, \Sigma}^{0}$, on obtient le foncteur de Quillen à gauche

$$
\hat{\iota}_{S}^{*}=\operatorname{Sus}_{T, \Sigma}^{0} \circ \iota_{S}^{*}: \mathbf{C p l}(\mathbf{P S h}(\mathrm{Et} / S, \Lambda)) \longrightarrow \operatorname{Spt}_{T}^{\Sigma}(\mathbf{C p l}(\mathbf{P S h}(\operatorname{Sm} / S, \Lambda))) .
$$

Son adjoint à droite est $\hat{\iota}_{S *}=\iota_{S *} \circ \mathrm{Ev}_{0}$. On en déduit aussitôt une adjonction

$$
\left(\mathrm{L} \hat{\imath}_{S}^{*}, \mathrm{R} \hat{\iota}_{S *}\right): \mathbf{D}^{\text {ét }}(S, \Lambda) \rightleftarrows \mathbf{D A}^{\text {ét }}(S, \Lambda) \text {. }
$$

Le but de ce paragraphe est d'établir le résultat suivant qu'on peut considérer comme une version relative (stable et sans transferts !) du théorème de rigidité de Suslin-Voevodsky (cf. [31, Th. 7.20 et Th. 9.35]).

Theoreme 4.1 - Soient $S$ un schéma et $\Lambda$ une $\mathbb{Z} / N \mathbb{Z}$-algèbre avec $N \in \mathbb{N}$ un entier inversible dans $\mathcal{O}_{S}$. On suppose qu'en tout point géométrique $\bar{x}$ de $S$ la p-dimension cohomologique ponctuelle de l'hensélisé strict de $S$ en $\bar{x}$ est finie si $p$ est un nombre premier divisant $N$. Alors, le foncteur $\mathrm{L} \hat{\imath}_{S}^{*}: \mathbf{D}^{\mathrm{e} t}(S, \Lambda) \longrightarrow \mathbf{D A}^{\mathrm{e} t}(S, \Lambda)$ est une équivalence de catégories.

L'hypothèse de dimension cohomologique dans l'énoncé ci-dessus est très faible. Elle est vérifiée lorsque $S$ est un corps ou un anneau de Dedekind. Elle est aussi vérifiée si la $p$-dimension cohomologie ponctuelle de $S$ est bornée pour tout diviseur premier de $N$. Dans [3, Expo. X], il est conjecturé qu'elle est vérifiée pour tout schéma excellent et les travaux de Gabber en cohomologie étale [14] permettent maintenant d'établir cette conjecture (cf. [25, Expo. XVIII-A, Th. 1.1 and Cor. 1.2]). Ceci dit, cette hypothèse servira dans la suite à travers le résultat suivant.

Lemme 4.2 - Soient $S$ un schéma et $\Lambda$ un anneau de coefficients. On suppose qu'en tout point géométrique $\bar{x}$ de $S$ les p-dimensions cohomologiques ponctuelles de l'hensélisé strict de $S$ en $\bar{x}$ sont uniformément bornées lorsque p parcourt les nombres premiers non inversibles dans $\Lambda$. Alors, pour tout $S$-schéma de type fini $X$, de morphisme structural $f: X \longrightarrow S$, le foncteur

$$
\mathrm{H}_{0} \circ \mathrm{R} f_{*}: \mathbf{C p l}(\mathbf{P S h}(\mathrm{Et} / X, \Lambda)) \longrightarrow \mathbf{S h}_{\text {ét }}(\mathrm{Et} / S, \Lambda)
$$

commute aux colimites filtrantes.

Demonstration En effet, soit $\left(K_{i}\right)_{i \in I}$ un système inductif filtrant de complexes de préfaisceaux sur $\mathrm{Et} / X$. On note $K=\operatorname{colim}_{i \in I} K_{i}$. On cherche à montrer que le morphisme canonique

$$
\operatorname{colim}_{i \in I} \mathrm{H}_{0}\left(\mathrm{R} f_{*} K_{i}\right) \longrightarrow \mathrm{H}_{0}\left(\mathrm{R} f_{*} K\right)
$$

est un isomorphisme. Par [5, Cor. 4.4.42], on dispose d'une équivalence de Quillen

$$
\text { (aét, oét) : } \mathbf{C p l}(\mathbf{P S h}(\mathrm{Et} / X, \Lambda)) \rightleftarrows \mathbf{C p l}\left(\mathbf{S h}_{\text {ét }}(\mathrm{Et} / X, \Lambda)\right)
$$

lorsqu'on muni la source de sa structure projective (ou injective) ét-locale et le but de sa structure injective, où les cofibrations sont les monomorphismes et les équivalences faibles sont les quasiisomorphismes de complexes de faisceaux étales. On ne restreint pas la généralité en supposant que les $K_{i}$ sont des complexes de faisceaux étales injectivement fibrants. On choisit également un quasi-isomorphisme de complexes de faisceaux étales $K \longrightarrow L$ avec $L$ injectivement fibrant. Il suffit maintenant de montrer que $\operatorname{colim}_{i \in I} f_{*} K_{i} \longrightarrow f_{*} L$ est un quasi-isomorphisme de complexes 
de faisceaux étales. La topologie étale étant quasi-compacte, le foncteur $f_{*}$ commute aux colimites filtrantes. On cherchera donc à montrer que $f_{*} K \longrightarrow f_{*} L$ est un quasi-isomorphisme de complexes de faisceaux étales.

Le résultat recherché se vérifie fibre par fibre dans le topos étale de $S$. Soient donc $\bar{x}$ un point géométrique de $S$ et $T=S_{\bar{x}}^{h s}$ l'hensélisé strict de $S$ en $\bar{x}$. Formons un carré cartésien

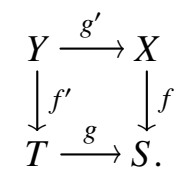

Il suffit alors de prouver que $f_{*}^{\prime} g^{\prime *} K \longrightarrow f_{*}^{\prime} g^{\prime *} L$ est un quasi-isomorphisme de complexes de faisceaux étales. Puisque $g^{\prime *} K \longrightarrow g^{\prime *} L$ est un quasi-isomorphisme, il suffira de voir que $f_{*}^{\prime} g^{\prime *} K \simeq$ $\mathrm{R} f_{*}^{\prime} g^{\prime *} K$ et de même pour $L$. Vu le Lemme 3.18, (a), il est donc suffisant de prouver que si $M$ est un complexe de faisceaux étales injectivement fibrant sur $\mathrm{Et} / X$, alors $g^{\prime *}(M)$ est au moins un complexe de préfaisceaux projectivement ét-fibrant.

Écrivons $M=\operatorname{colim}_{n \in \mathbb{N}} \sigma_{\leq n} M$, avec $\sigma_{\leq n}$ la troncation bête. En appliquant une deuxième fois le Lemme 3.18, (a), on voit qu'il suffit de montrer que $g^{\prime *}\left(\sigma_{\leq n} M\right)$ est projectivement ét-fibrant pour tout $n$. Puisque $M$ est injectivement fibrant, les faisceaux $M_{r}$, pour $r \in \mathbb{Z}$, sont injectifs. En particulier, $\mathrm{H}_{\mathrm{et}}^{s}\left(U, M_{r}\right)=0$ pour tout $U \in \mathrm{Et} / X$ et $s \in \mathbb{N}-\{0\}$. En utilisant [2, Expo. VII, Th. 5.7] (et le fait que $g$ est pro-étale) on déduit aussitôt que les faisceaux $g^{* *} M_{r}$ sont acycliques. En particulier, ils sont projectivement ét-fibrants, vus comme complexes de préfaisceaux sur Et $/ Y$. Une récurrence simple montre alors que les complexes de préfaisceaux $\sigma_{\geq-m} g^{\prime *}\left(\sigma_{\leq n} M\right)$ sont projectivement ét-fibrants pour tout $m \in \mathbb{N}$. En écrivant $g^{\prime *}\left(\sigma_{\leq n} M\right)=\lim _{m \in \mathbb{N}} \sigma_{\geq-m} g^{\prime *}\left(\sigma_{\leq n} M\right)$, il s'ensuit que $g^{\prime *}\left(\sigma_{\leq n} M\right)$ est lui-même projectivement ét-fibrant. Ceci termine la preuve du lemme.

Le point de départ de notre preuve du Théorème 4.1 est un résultat de rigidité à la SuslinVoevodsky obtenu par Röndigs et Østvær [43]. Nous présentons ici une forme remaniée de ce résultat, mieux adaptée à nos besoins. Pour cela, nous devons reprendre quelques arguments de [43]. Soient $k$ un corps algébriquement clos et $C$ une $k$-courbe affine et lisse. Le groupe $\operatorname{Div}(C)$, des diviseurs de $C$, est le $\mathbb{Z}$-module librement engendré par l'ensemble $C(k)$ des points fermés de $C$. Un tel point $c \in C(k)$ induit un morphisme de préfaisceaux $c: \operatorname{Spec}(k) \otimes \Lambda \longrightarrow C \otimes \Lambda$. Par linéarité, on déduit un homomorphisme de groupes abéliens

$$
\operatorname{Div}(C) \longrightarrow \operatorname{hom}_{\mathbf{D A}^{\mathrm{et}}(k, \Lambda)}(\operatorname{Spec}(k) \otimes \Lambda, C \otimes \Lambda) .
$$

(Ci-dessus, on désigne par abus de notation $X \otimes \Lambda$ le motif dans $\mathbf{D A}^{\text {ét }}(k, \Lambda)$ associé à $X \in \mathrm{Sm} / k$, i.e., le $T$-spectre symétrique $\operatorname{Sus}_{T, \Sigma}^{0}(X \otimes \Lambda)$.) Soit $\bar{C}$ une compactification lisse de $C$ (unique à un isomorphisme près). On dispose alors d'une surjection $\operatorname{Div}(C) \longrightarrow \operatorname{Pic}(\bar{C}, Z)$, où $\operatorname{Pic}(\bar{C}, Z)$ est le groupe de Picard relatif de la paire $(\bar{C}, Z)$ avec $Z=\bar{C}-C$ (cf. [31, Def. 7.10]). L'énoncé suivant ne fait aucune hypothèse sur l'anneau $\Lambda$.

Lemme 4.3 - L'homomorphisme (28) se factorise à travers $\operatorname{Pic}(\bar{C}, Z)$.

Demonstration Il s'agit d'une conséquence immédiate de [43, Th. 4.6]. En effet, il existe un foncteur évident $\mathbf{S H}(k) \longrightarrow \mathbf{D A}^{\text {ét }}(k, \Lambda)$ qui envoie $X_{+}$sur $X \otimes \Lambda$ pour tout $X \in \mathrm{Sm} / k$. (C'est le foncteur (164) (avec $\tau=$ ét) composé avec le foncteur de changement de topologie aét : $\mathbf{S H}(S) \longrightarrow \mathbf{S H}^{\mathrm{t}}(S)$.) De plus, on a un triangle commutatif

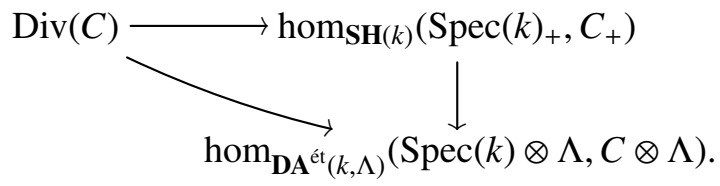

Or, [43, Th. 4.6] affirme que la flèche horizontale se factorise à travers $\operatorname{Pic}(\bar{C}, Z)$.

Corollaire 4.4 - Soient $k$ un corps séparablement clos et $\Lambda$ une $\mathbb{Z} / N \mathbb{Z}$-algèbre avec $N \in \mathbb{N}$ un entier inversible dans $k$. Soient $X$ un $k$-schéma quasi-projectif, lisse et connexe, et $x_{0}, x_{1} \in X(k)$. 
Alors, pour tout objet $M \in \mathbf{D A}^{\mathrm{ét}}(k, \Lambda)$, les deux homomorphismes

$$
x_{0}^{*}, x_{1}^{*}: \operatorname{hom}_{\mathbf{D A}^{\text {et }(k, \Lambda)}}(X \otimes \Lambda, M) \longrightarrow \operatorname{hom}_{\mathbf{D A}^{\text {et }(k, \Lambda)}}(\operatorname{Spec}(k) \otimes \Lambda, M)
$$

sont égaux.

Demonstration Soit $l / k$ une extension finie et donc nécessairement totalement inséparable. On désigne par $e: \operatorname{Spec}(l) \longrightarrow \operatorname{Spec}(k)$ le morphisme associé. D'après le Théorème 3.9 (joint à $[4$, Prop. 2.1.163]), le foncteur $e^{*}: \mathbf{D A}^{\text {ét }}(k, \Lambda) \longrightarrow \mathbf{D A}^{\text {ét }}(l, \Lambda)$ est une équivalence de catégories. On peut donc remplacer $k, X$ et $M$ par $l, X \otimes_{k} l$ et $e^{*} M$. En utilisant la Proposition 3.20, on peut même remplacer $k, X$ et $M$ par $\bar{k}, X \otimes_{k} \bar{k}$ et le «pull-back » de $M$ suivant $\operatorname{Spec}(\bar{k}) \longrightarrow \operatorname{Spec}(k)$, avec $\bar{k}$ une clôture algébrique de $k$. Autrement dit, on ne restreint pas la généralité en supposant que $k$ est algébriquement clos.

Par ailleurs, on peut aussi supposer que $X$ est une courbe affine, lisse et connexe que l'on notera $C$. (En effet, il existe une telle courbe $C \subset X$ qui contient les points $x_{0}$ et $x_{1}$.) Le diviseur $\left[x_{0}\right]-\left[x_{1}\right]$ appartient au noyau de l'homomorphisme $\operatorname{deg}: \operatorname{Pic}(\bar{C}, Z) \longrightarrow \mathbb{Z}$, que l'on note $\operatorname{Pic}^{0}(\bar{C}, Z)$. D'après le Lemme 4.3, il suffit alors de prouver que la classe de $\left[x_{0}\right]-\left[x_{1}\right]$ est nulle dans $\operatorname{Pic}^{0}(\bar{C}, Z) \otimes \mathbb{Z} / N \mathbb{Z}$. Mais ce groupe est lui-même nul puisque $\operatorname{Pic}^{0}(\bar{C}, Z)$ est divisible. En effet, ce dernier s'identifie au groupe des $k$-points d'une variété semi-abélienne, la variété d'Albanese de $C$. Le corollaire est démontré.

Nous arrivons maintenant au théorème de rigidité de Röndigs et Østvær [43, Th. 1.1] adapté au contexte qui nous intéresse.

Proposition 4.5 - Soient $k$ un corps séparablement clos et $\Lambda$ une $\mathbb{Z} / N \mathbb{Z}$-algèbre avec $N \in$ $\mathbb{N}$ un entier inversible dans $k$. Soit $K / k$ une extension avec $K$ séparablement clos et notons $e$ : $\operatorname{Spec}(K) \longrightarrow \operatorname{Spec}(k)$ le morphisme associé. Alors, le foncteur $e^{*}: \mathbf{D A}^{\text {ét }}(k, \Lambda) \longrightarrow \mathbf{D A}^{\text {ét }}(K, \Lambda)$ est pleinement fidèle. ${ }^{(6)}$

Demonstration Soient $M$ et $P$ des objets de $\mathbf{D A}{ }^{\text {ét }}(k, \Lambda)$. Il s'agit de montrer que l'homomorphisme

$$
\operatorname{hom}_{\mathbf{D A}^{\mathrm{et}}(k, \Lambda)}(M, P) \longrightarrow \operatorname{hom}_{\mathbf{D} \mathbf{A}^{\mathrm{et}}(K, \Lambda)}\left(e^{*} M, e^{*} P\right)
$$

est une bijection. D'après la Proposition 3.19, la catégorie $\mathbf{D} A^{\text {ét }}(k, \Lambda)$ est compactement engendrée. Étant donné que $e^{*}$ commute aux sommes infinies, on voit que si l'homomorphisme (29) est inversible pour $M$ compact il l'est aussi pour tout $M$. On peut alors supposer que $M$ est un objet compact et donc fortement dualisable (voir [39] ou encore [6, Lem. 1.3.29]). Puisque $e^{*}$ est un foncteur monoïdal, on peut même supposer que $M=\operatorname{Sus}_{T, \Sigma}^{0}(\operatorname{Spec}(k) \otimes \Lambda)$, l'objet unité de $\mathbf{D A}^{\text {ét }}(k, \Lambda)$, quitte à remplacer $P \operatorname{par} \underline{\operatorname{Hom}}(M, P)$.

Soient $l / k$ une extension finie totalement inséparable de $k$ et $L / K$ l'extension composée de $K$ et $l$. En utilisant le Théorème 3.9 (joint à [4, Prop. 2.1.163]) on voit qu'on peut remplacer $k$ et $K$ par $l$ et $L$. En utilisant la Proposition 3.20, on peut même remplacer $k$ par sa clôture algébrique. En particulier, on ne restreint pas la généralité en supposant que l'extension $K / k$ est séparable, i.e., que $K$ est l'union filtrante de ses sous- $k$-algèbres lisses.

Le reste de la preuve est une application standard du Corollaire 4.4. Rappelons qu'on cherche à montrer que l'homomorphisme

$$
\operatorname{hom}_{\mathbf{D A}^{\text {et }(k, \Lambda)}}(\operatorname{Spec}(k) \otimes \Lambda, P) \longrightarrow \operatorname{hom}_{\mathbf{D A}^{\text {st }}(K, \Lambda)}\left(\operatorname{Spec}(K) \otimes \Lambda, e^{*} P\right)
$$

est une bijection. L'injectivité est facile. Soit $\alpha \in \operatorname{hom}_{\mathbf{D A}^{\text {et }}(k, \Lambda)}(\operatorname{Spec}(k) \otimes \Lambda, P)$ un élément du noyau de (30). D'après la Proposition 3.20, il existe une sous- $k$-algèbre lisse $A \subset K$ telle que l'image de $\alpha$ est dans le noyau de l'homomorphisme

$$
\operatorname{hom}_{\mathbf{D A}^{\text {et }(k, \Lambda)}}(\operatorname{Spec}(k) \otimes \Lambda, P) \longrightarrow \operatorname{hom}_{\mathbf{D A}}{ }^{\text {et }(A, \Lambda)}\left(\operatorname{Spec}(A) \otimes \Lambda, e_{A}^{*} P\right)
$$

6. En fait, d'après le Théorème 4.1, le foncteur $e^{*}$ est même une équivalence de catégories. La situation est différente de celle de [43, Th. 1.1] où le foncteur analogue n'est pas essentiellement surjectif. 
avec $e_{A}: \operatorname{Spec}(A) \longrightarrow \operatorname{Spec}(k)$ le morphisme évident. Or, l'homomorphisme (31) admet des retractions induites par les $k$-points de la $k$-variété lisse $\operatorname{Spec}(A)$. (De tels $k$-points existent puisque $k$ est séparablement clos.) Il en découle que $\alpha=0$.

Pour montrer la surjectivité de (30) on fixe $\beta \in \operatorname{hom}_{\mathbf{D A}^{\mathrm{et}}(K, \Lambda)}\left(\operatorname{Spec}(K) \otimes \Lambda, e^{*} P\right)$. D'après la Proposition 3.20, il existe une sous- $k$-algèbre lisse $B \subset K$ et $\beta_{0} \in \operatorname{hom}_{\mathbf{D A}^{\text {et }}(B, \Lambda)}\left(\operatorname{Spec}(B) \otimes \Lambda, e_{B}^{*} P\right)$ qui est un antécédant de $\beta$ par le morphisme évident. Dans la suite, on note $X=\operatorname{Spec}(B)$ et $f=e_{B}: X \longrightarrow \operatorname{Spec}(k)$ le morphisme évident. On note aussi $f_{K}: X_{K} \longrightarrow \operatorname{Spec}(K)$ le changement de base de $f$ suivant $e$ et $e^{\prime}: X_{K} \longrightarrow X$ la projection évidente. On a $e \circ f_{K}=f \circ e^{\prime}$.

Fixons un $k$-point $x \in X(k)$. Il induit un $K$-point $x_{K} \in X_{K}(K)$. D'autre part, l'inclusion $B \hookrightarrow K$ induit un $K$-point « générique » $\eta \in X_{K}(K)$. D'après le Corollaire 4.4 (et un petit jeu d'adjonction), les deux morphismes

$$
x_{K}^{*}, \eta^{*}: \operatorname{hom}_{\mathbf{D A}^{\text {et }}\left(X_{K}, \Lambda\right)}\left(X_{K} \otimes \Lambda, f_{K}^{*} e^{*} P\right) \longrightarrow \operatorname{hom}_{\mathbf{D A}^{\text {et }}(K, \Lambda)}\left(\operatorname{Spec}(K) \otimes \Lambda, e^{*} P\right)
$$

coïncident. Or, il est clair que $\beta=\eta^{*} e^{\prime *}\left(\beta_{0}\right)$. On en déduit alors que $\beta=x_{K}^{*} e^{\prime *}\left(\beta_{0}\right)=e^{*} x^{*} \beta_{0}$. Autrement dit, $\beta$ est égal à l'image de $x^{*} \beta_{0}$ par l'homomorphisme (30).

La discussion précédente nous permettra d'établir le Théorème 4.1 lorsque $S$ est le spectre d'un corps (vérifiant une condition technique dont on se débarassera plus tard).

Lemme 4.6 - Le Théorème 4.1 est vrai lorsque $S=\operatorname{Spec}(k)$ avec $k$ un corps de p-dimension cohomologique finie pour tout nombre premier p divisant $N$.

Demonstration Pour la preuve nous travaillerons avec les $T$-spectres non symétriques en complexes de préfaisceaux sur $\mathrm{Sm} / k$. Ceci est possible par [5, Th. 4.3.79]. En effet, l'analogue de [5, Lem. 4.5.65] est vrai dans $\mathbf{D A}{ }^{\text {eff, ét }}(k, \Lambda)^{(7)}$ et le foncteur $\underline{\operatorname{Rom}}\left(T_{k},-\right)=\operatorname{Rhom}\left(\left(\mathbb{P}_{k}^{1}, \infty\right),-\right)$ commute aux compositions transfinies (cf. [5, Hyp. 4.3.56]) sous l'hypothèse de finitude cohomologique de l'énoncé.

Nous divisons la preuve en trois étapes. À la fin de la troisième étape, nous obtiendrons que le foncteur $\hat{R}_{k *}$ est une équivalence de catégories ce qui démontre bien le résultat recherché.

Étape A : Soit $\mathbf{E}$ un $T$-spectre (non symétrique) en complexes de préfaisceaux sur $\mathrm{Sm} / k$ et supposons qu'il est projectivement stablement $\left(\mathbb{A}^{1}\right.$, ét)-fibrant. Soit $K / k$ une extension séparable de type fini. Fixons des clôtures séparables $k^{s e p}$ et $K^{s e p}$ de $k$ et $K$, ainsi qu'un morphisme de $k$-extensions $k^{\text {sep }} \hookrightarrow K^{\text {sep }}$. Pour $n \in \mathbb{N}$, considérons le morphisme de complexes

$$
\mathbf{E}_{n}\left(k^{s e p}\right) \longrightarrow \mathbf{E}_{n}\left(K^{s e p}\right) .
$$

(Étant donnés un préfaisceau $F$ sur $\mathrm{Sm} / k$ et une $k$-algèbre $\mathcal{A}$ qui est la réunion filtrante de ses sous- $k$-algèbres lisses, nous notons $F(\mathcal{A})$ la colimite filtrante des $F(\operatorname{Spec}(A))$ où $A$ parcourt les sous-algèbres lisses de $\mathcal{A}$.) Sur le $m$-ième module d'homologie (pour $m \in \mathbb{Z}$ ) le morphisme (32) s'identifie, aux isomorphismes canoniques près, avec l'homomorphisme

$$
\operatorname{hom}_{\mathbf{D A}^{\mathrm{et}}\left(k^{s e p}, \Lambda\right)}\left(\operatorname{Sus}_{T}^{n}\left(\Lambda_{c s t}[m]\right), \mathbf{E}_{\left.\mid k^{s e}\right)}\right) \longrightarrow \operatorname{hom}_{\mathbf{D A}^{\mathrm{et}}\left(K^{s e p}, \Lambda\right)}\left(\operatorname{Sus}_{T}^{n}\left(\Lambda_{c s t}[m]\right), \mathbf{E}_{\mid K^{s e p}}\right) .
$$

(Ci-dessus, $\Lambda_{c s t}$ est le préfaisceau constant de valeur $\Lambda$, et $\mathbf{E}_{\mid k^{s e p}}$ et $\mathbf{E}_{\mid K^{s e p}}$ sont les «pull-back $\gg$ de $\mathbf{E}$ suivant les morphismes associés aux extensions $k^{\text {sep }} / k$ et $K^{s e p} / k$.) Pour obtenir cette identification, nous avons besoin de la Proposition 3.20 (d'où l'utilité de la finitude de certaines $p$-dimensions cohomologiques de $k$ ). Elle permet d'écrire (33) comme une colimite d'homomorphismes qu'on identifie, par adjonction, avec les homomorphismes $\mathrm{H}_{m}\left(\mathbf{E}_{n}(l)\right) \longrightarrow \mathrm{H}_{m}\left(\mathbf{E}_{n}(L)\right)$ où $l / k$ est une extension finie contenue dans $k^{\text {sep }}$ et $L$ est une $l$-algèbre lisse contenue dans $K^{s e p}$. Ceci dit, on peut maintenant appliquer la Proposition 4.5 pour déduire que le morphisme de complexes (32) est un quasi-isomorphisme.

Considérons à présent le morphisme de complexes de préfaisceaux

$$
\iota_{k}^{*} \iota_{k *}\left(\mathbf{E}_{n}\right) \longrightarrow \mathbf{E}_{n} .
$$

7. Notons au passage que la preuve de [5, Lem. 4.5.65] contient une erreur. En effet, le groupe $\mathbb{G} \ell_{S}(3)$ n'agit pas sur $\operatorname{Cof}\left(\mathbb{G m}_{S}^{3} \otimes \mathbb{1} \rightarrow \mathbb{A}_{S}^{3} \otimes \mathbb{1}\right)$. Il agit toutefois sur $\operatorname{Cof}\left(\left(\mathbb{A}_{S}^{3}-0_{S}\right) \otimes \mathbb{1} \rightarrow \mathbb{A}_{S}^{3} \otimes \mathbb{1}\right)$ et ceci est suffisant pour conclure puisque cette cofibre s'identifie d'une manière $\Sigma_{3}$-équivariante à la précédente modulo un shift. 
D'après la discussion précédente, ce morphisme est un quasi-isomorphisme lorsqu'on l'évalue sur la clôture séparable $K^{\text {sep }}$ d'une extension séparable $K / k$. (En effet, on a un isomorphisme canonique $\left(\iota_{k}^{*} \iota_{k *}\left(\mathbf{E}_{n}\right)\right)\left(K^{s e p}\right) \simeq \mathbf{E}_{n}\left(k^{s e p}\right)$.) Par ailleurs, les complexes de préfaisceaux $\mathbf{E}_{n}$ et $\iota_{k}^{*} \iota_{k *}\left(\mathbf{E}_{n}\right)$ sont tous les deux $\mathbb{A}^{1}$-locaux. Pour le premier, c'est clair puisque $\mathbf{E}_{n}$ est supposé ( $\mathbb{A}^{1}$, ét)-fibrant, et pour le second, on utilise le Lemme 4.7 ci-dessous. En considérant le cône du morphisme (34) et en utilisant le Lemme 4.8 ci-dessous on déduit en fin de compte que (34) est une équivalence ét-locale.

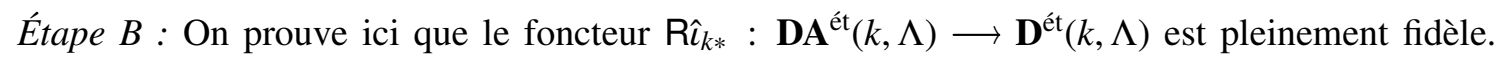
Soient $\mathbf{E}$ et $\mathbf{F}$ des $T$-spectres (non symétriques) en complexes de préfaisceaux sur $\mathrm{Sm} / k$, et supposons qu'ils sont projectivement stablement ( $\mathbb{A}^{1}$, ét)-fibrants. Supposons aussi que $\mathbf{E}$ est projectivement cofibrant niveau par niveau. On cherche à calculer $\operatorname{hom}_{\mathbf{D A}^{\text {et }}(k, \Lambda)}(\mathbf{E}, \mathbf{F})$. Pour $r \in \mathbb{N}$, on note $\mathbf{E}^{(r)}$ le $T$-spectre défini par :

$$
\left(\mathbf{E}^{(r)}\right)_{n}=\left\{\begin{array}{ccc}
\mathbf{E}_{n} & \text { si } & n \leq r \\
T_{k}^{\otimes n-r} \otimes \mathbf{E}_{r} & \text { si } & n \geq r+1 .
\end{array}\right.
$$

(Les morphismes d'assemblage sont ceux que l'on pense.) On a alors des morphismes évidents $\mathbf{E}^{(r)} \longrightarrow \mathbf{E}^{(r+1)}$ et on peut écrire $\mathbf{E}=\operatorname{colim}_{r \in \mathbb{N}} \mathbf{E}^{(r)}$. Or, les $\mathbb{N}$-colimites préservent les équivalences $\left(\mathbb{A}^{1}\right.$, ét)-locales. (Pour démontrer cela, on peut utiliser [5, Lem. 4.2.69] et le fait que les $\mathbb{N}$-colimites préservent les quasi-isomorphismes de complexes de préfaisceaux.) On en déduit que la colimite des $\mathbf{E}^{(r)}$ coïncide avec leur colimite homotopique. Autrement dit, on a un isomorphisme canonique

$$
\mathbf{E} \simeq \operatorname{hocolim}_{r \in \mathbb{N}} \mathbf{E}^{(r)}
$$

dans la catégorie triangulée $\mathbf{D} \mathbf{A}^{\text {ét }}(k, \Lambda)$. Ceci fournit un triangle distingué

$$
\bigoplus_{r \in \mathbb{N}} \mathbf{E}^{(r)} \longrightarrow \bigoplus_{r \in \mathbb{N}} \mathbf{E}^{(r)} \longrightarrow \mathbf{E} \longrightarrow
$$

On en déduit facilement une suite exacte courte (de Milnor)

$$
0 \rightarrow \lim _{r \in \mathbb{N}}{ }^{1} \operatorname{hom}_{\mathbf{D A}^{\text {et }}(k, \Lambda)}\left(\mathbf{E}^{(r)}, \mathbf{F}[-1]\right) \rightarrow \operatorname{hom}_{\mathbf{D A}}{ }^{\text {et }(k, \Lambda)}(\mathbf{E}, \mathbf{F}) \rightarrow \lim _{r \in \mathbb{N}} \operatorname{hom}_{\mathbf{D A}}{ }^{\text {et }(k, \Lambda)}\left(\mathbf{E}^{(r)}, \mathbf{F}\right) \rightarrow 0
$$

Il suffira alors de montrer que les morphismes de transition dans les systèmes projectifs

$$
\left(\operatorname{hom}_{\mathbf{D A}^{\text {et }}(k, \Lambda)}\left(\mathbf{E}^{(r)}, \mathbf{F}\right)\right)_{r \in \mathbb{N}} \quad \text { et } \quad\left(\operatorname{hom}_{\mathbf{D A}^{\text {et }}(k, \Lambda)}\left(\mathbf{E}^{(r)}, \mathbf{F}[-1]\right)\right)_{r \in \mathbb{N}}
$$

sont des isomorphismes. En effet, si c'était le cas, on obtient des isomorphismes canoniques

$$
\operatorname{hom}_{\mathbf{D A}}{ }^{\text {et }(k, \Lambda)}(\mathbf{E}, \mathbf{F}) \simeq \operatorname{hom}_{\mathbf{D A}^{\text {et }}(k, \Lambda)}\left(\mathbf{E}^{(0)}, \mathbf{F}\right) \simeq \operatorname{hom}_{\mathbf{D A}^{\text {eff, ét }(k, \Lambda)}}\left(\mathbf{E}_{0}, \mathbf{F}_{0}\right) \text {. }
$$

Or, d'après l'étape $\mathrm{A}$, le morphisme de counité $\iota_{k}^{*} \iota_{k *} \mathbf{F}_{0} \longrightarrow \mathbf{F}_{0}$ est une équivalence ét-locale. On en déduit un isomorphisme canonique hom $\mathbf{D A}^{\text {eff, et }}\left(\mathbf{E}_{0}, \mathbf{F}_{0}\right) \simeq \operatorname{hom}_{\mathbf{D}^{\mathrm{st}}(k, \Lambda)}\left(\iota_{k *} \mathbf{E}_{0}, \iota_{k *} \mathbf{F}_{0}\right)$. Il en découle que l'homomorphisme

$$
\operatorname{hom}_{\mathbf{D A}^{\text {ét }(k, \Lambda)}}(\mathbf{E}, \mathbf{F}) \longrightarrow \operatorname{hom}_{\mathbf{D}^{\mathrm{et}}(k, \Lambda)}\left(\iota_{k *} \mathbf{E}_{0}, \iota_{k *} \mathbf{F}_{0}\right)
$$

est inversible. Et c'est ce qu'on cherchait à démontrer dans cette étape.

Il nous reste à vérifier que les systèmes projectifs (36) sont constants. Bien entendu, il suffit de considérer le premier système. On dispose d'un morphisme canonique $\operatorname{Sus}_{T}^{r}\left(\mathbf{E}_{r}\right) \longrightarrow \mathbf{E}^{(r)}$ qui est un isomorphisme à partir du $r$-ième niveau. $C^{\prime}$ est donc une équivalence $\left(\mathbb{A}^{1}\right.$, ét)-locale stable d'après [5, Lem. 4.3.59]. Ceci fournit des isomorphismes canoniques

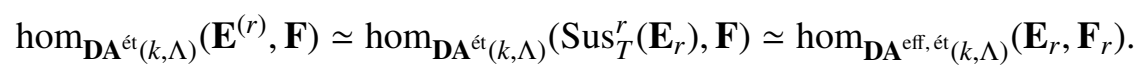

Modulo ces identifications, le $r$-ième morphisme de transition du système projectif (36) est donné par

$$
\operatorname{hom}_{\mathbf{D A}^{\mathrm{eff}, \mathrm{et}}(k, \Lambda)}\left(\mathbf{E}_{r+1}, \mathbf{F}_{r+1}\right) \longrightarrow \operatorname{hom}_{\mathbf{D A}^{\mathrm{eff}, \mathrm{et}}(k, \Lambda)}\left(\underline{\operatorname{Hom}}\left(T_{k}, \mathbf{E}_{r+1}\right), \underline{\operatorname{Hom}}\left(T_{k}, \mathbf{F}_{r+1}\right)\right)
$$

composé avec l'isomorphisme

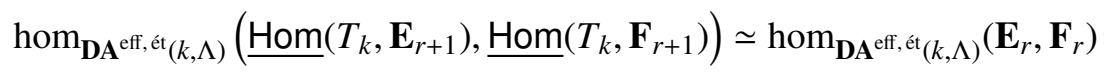


déduit du fait que $\mathbf{E}$ et $\mathbf{F}$ sont des $\Omega$-spectres. Pour terminer, il nous reste donc à voir que (37) est un isomorphisme. Pour cela, on utilise l'étape A pour réécrire ce morphisme de la manière suivante :

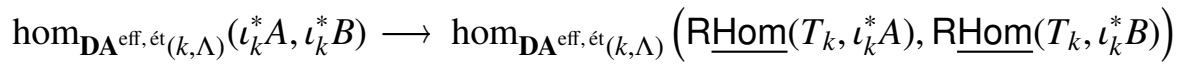

avec $A=\iota_{k *} \mathbf{E}_{r+1}$ et $B=\iota_{k *} \mathbf{F}_{r+1}$. Or, il est immédiat de voir que $\operatorname{RHom}\left(T_{k}, \iota_{k}^{*} A\right) \simeq \iota_{k}^{*}(A(-1))[-2]$ et de même pour $B$. (Ici, $-(-1)$ désigne le twist de Tate usuel pour les faisceaux étales.) Ceci permet de conclure.

Étape $C$ : Pour terminer la preuve du lemme, il nous reste à montrer que $\mathrm{R} \hat{\iota}_{k *}$ est essentiellement surjectif. Pour cela, on fixe un complexe de préfaisceaux $K$ sur Et $/ k$. On ne restreint pas la généralité en supposant que $K$ est projectivement cofibrant. Pour tout $n \in \mathbb{N}$, on choisit une équivalence ét-locale $\iota_{k}^{*}(K(n))[2 n] \longrightarrow \mathbf{L}_{n}$ avec $\mathbf{L}_{n}$ un complexe de préfaisceaux projectivement ét-fibrant sur $\mathrm{Sm} / k$. Alors, d'après le Sous-lemme 4.7, $\mathbf{L}_{n}$ est projectivement ( $\mathbb{A}^{1}$, ét)-fibrant. De plus, il est aussi projectivement cofibrant. Par ailleurs, dans $\mathbf{D A}{ }^{\text {eff,ét }}(k, \Lambda)$, on a un isomorphisme

$$
\iota_{k}^{*}(K(n))[2 n] \simeq \mathrm{R} \underline{\operatorname{Hom}}\left(T_{k}, \iota_{k}^{*}(K(n+1))[2 n+2]\right) .
$$

Il existe alors un quasi-isomorphisme de complexes de préfaisceaux $\mathbf{L}_{n} \longrightarrow \underline{\operatorname{Hom}}\left(T_{k}, \mathbf{L}_{n+1}\right)$ qui réalise cet isomorphisme. Par adjonction, on déduit un morphisme $\gamma_{n}: T_{k} \otimes \mathbf{L}_{n} \longrightarrow \mathbf{L}_{n+1}$. Le Tspectre (non symétrique) $\mathbf{L}=\left(\mathbf{L}_{n}, \gamma_{n}\right)_{n \in \mathbb{N}}$ est projectivement stablement ( $\mathbb{A}^{1}$, ét)-fibrant. De plus, $\mathrm{R} \hat{\iota}_{k *}(\mathbf{L})=\iota_{k *} \mathbf{L}_{0}$ est isomorphe à $K$ dans $\mathbf{D}^{\text {ét }}(k, \Lambda)$ par construction.

Sous-LEMme 4.7 - Soient $S$ un schéma et $\Lambda$ une $\mathbb{Z} / N \mathbb{Z}$-algèbre avec $N \in \mathbb{N}$ un entier inversible dans $\mathcal{O}_{S}$. On suppose qu'en tout point géométrique $\bar{x}$ de $S$ la p-dimension cohomologique ponctuelle du hensélisé strict de $S$ en $\bar{x}$ est finie si p est un nombre premier divisant $N$. Soit $K$ un complexe de préfaisceaux sur $\mathrm{Et} / S$. Alors, $\iota_{S}^{*}(K)$ est un objet $\mathbb{A}^{1}$-local de la catégorie $\mathbf{H o}_{\text {ét }}(\mathbf{C p l}(\mathbf{P S h}(\operatorname{Sm} / S, \Lambda)))$.

Demonstration Il s'agit essentiellement d'une reformulation de l'invariance par homotopie de la cohomologie étale [3, Expo. XV, Cor. 2.2]. En effet, dire que $\iota_{S}^{*}(K)$ est $\mathbb{A}^{1}$-local revient à dire que l'homomorphisme

$$
\mathrm{H}_{\dot{e t}}^{\bullet}\left(X, \iota_{S}^{*}(K)_{\mid \mathrm{Et} / X}\right) \longrightarrow \mathrm{H}_{\dot{\mathrm{et}}}^{\cdot}\left(\mathbb{A}_{X}^{1}, \iota_{S}^{*}(K)_{\mid \mathrm{Et} / \mathbb{A}_{X}^{1}}\right)
$$

est inversible pour tout $X \in \mathrm{Sm} / S$. (On utilise ici implicitement [5, Th. 4.4.60] appliqué au prémorphisme de sites induit par l'inclusion $\mathrm{Et} / X \hookrightarrow \mathrm{Sm} / S$ pour s'assurer que la restriction à $\mathrm{Et} / X$ d'un complexe de préfaisceaux projectivement ét-fibrant sur $\mathrm{Sm} / S$ est encore projectivement étfibrant.) Or, il est clair que $\iota_{S}^{*}(K)_{\mid \mathrm{Et} / \mathbb{A}_{X}^{1}}=p^{*}\left(\iota_{S}^{*}(K)_{\mid \mathrm{Et} / X}\right)$ où $p: \mathbb{A}_{X}^{1} \longrightarrow X$ est la projection évidente et $p^{*}: \mathbf{P S h}(\mathrm{Et} / X, \Lambda) \longrightarrow \mathbf{P S h}\left(\operatorname{Et} / \mathbb{A}_{X}^{1}, \Lambda\right)$ le foncteur image inverse. Avec $L=\iota_{S}(K)_{\mid \mathrm{Et} / X}$, il nous faut donc prouver que l'homomorphisme $\mathrm{H}_{\dot{e t}}^{\cdot}(X, L) \longrightarrow \mathrm{H}_{\text {et }}^{\cdot}\left(\mathbb{A}_{X}^{1}, p^{*}(L)\right)$ est inversible. Il est en fait suffisant de prouver que le morphisme d'unité $L \longrightarrow \mathrm{R} p_{*} p^{*}(L)$ est un isomorphisme dans $D^{\text {ét }}(X, \Lambda)$. En utilisant le Lemme 4.2 et en écrivant $L=\operatorname{colim}_{n \in \mathbb{N}} \sigma_{\leq n}\left(\tau_{\geq-n}(L)\right)$, on se ramène au cas où $L$ est borné. Une récurrence immédiate nous ramène au cas où $L$ est concentré en degré zéro. On peut maintenant appliquer [3, Expo. XV, Cor. 2.2] pour conclure.

Sous-LEMme 4.8 - Soit $k$ un corps et supposons que les p-dimensions cohomologiques de $k$ sont uniformément bornées lorsque p parcourt les nombres premiers non inversibles dans $\Lambda$. Soit $M$ un complexe de préfaisceaux sur $\mathrm{Sm} / k$. Supposons que $M$ est $\mathbb{A}^{1}$-local et que pour toute extension séparable de type fini $K / k, M\left(K^{\text {sep }}\right) \simeq 0$ dans $\mathbf{D}(\Lambda)$. (Bien entendu, $K^{\text {sep }}$ est une clôture séparable de $K$.) Alors, $M \simeq 0$ dans $\mathbf{D A}{ }^{\text {eff, ét }}(k, \Lambda)$.

Demonstration On peut supposer que $M$ est projectivement $\left(\mathbb{A}^{1}\right.$, ét)-fibrant. La finitude des $p$ dimensions cohomologiques assure que $M(K) \simeq \mathrm{R} \Gamma\left(\mathrm{Gal}\left(K^{\text {sep }} / K\right), M\left(K^{\text {sep }}\right)\right)$ ce qui montre que le complexe $M(K)$ est contractile. Dans la suite, nous allons considérer $M$ comme un objet de $\mathbf{D A}^{\mathrm{eff}}(k, \Lambda)$, la catégorie des motifs sans transferts et pour la topologie Nisnevich, et nous montrerons que $M$ est isomorphe à l'objet nul dans cette catégorie. 
Par le Théorème de $\mathbb{A}^{1}$-connexité stable de Morel [34], les foncteurs de troncations de la $t$ structure usuelle sur $\mathbf{D}\left(\mathbf{S h}_{\mathrm{Nis}}(\mathrm{Sm} / k, \Lambda)\right)$ préservent la sous-catégorie $\mathbf{D A}{ }^{\text {eff }}(k, \Lambda)$. Ils induisent donc une $t$-structure sur cette dernière. Elle est appelée la $t$-structure homotopique et son cœur est canoniquement équivalent à la catégorie des faisceaux Nisnevich strictement invariants par homotopie. Il suffit de montrer que les faisceaux Nisnevich $\mathrm{h}_{i}(M)=\mathrm{a}_{\mathrm{Nis}} \mathrm{H}_{i}(-, M)$ sont nuls pour tout $i \in \mathbb{Z}$. D'après ce qui précède, on sait que $\mathrm{h}_{i}(M)(K)=0$ pour toute extension de type finie $K$ de $k$. Or, si $F$ est un faisceau Nisnevich strictement invariant par homotopie, les morphismes de restrictions $F(X) \longrightarrow F(U)$ sont injectifs pour toute immersion ouverte d'image dense $U \hookrightarrow X$. (On se ramène pour cela au cas où $Z=X-U$ est lisse de faisceau normal libre de rang $d \geq 1$. On a alors par pureté une suite exacte

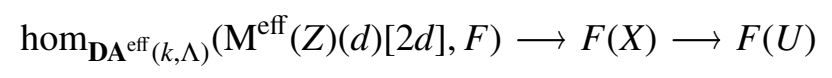

avec $\mathrm{M}^{\text {eff }}(Z)$ le motif effectif de $Z$, i.e., le préfaisceau $Z \otimes \Lambda$ vu comme objet de $\mathbf{D A}{ }^{\text {eff }}(k, \Lambda)$. Le membre de gauche de cette suite exacte est nul. En effet, $\mathrm{M}^{\text {eff }}(Z)(d)[2 d] \simeq\left[Z \times_{k}\left(\mathbb{G m}_{k}, 1\right)^{\wedge d}\right] \otimes \Lambda[d]$ est strictement positif pour la $t$-structure homotopique alors que $F$ est dans le cœur.) Ceci entraîne que les $\mathrm{h}_{i}(M)$ sont nuls. Le sous-lemme est démontré.

Notons le résultat suivant qui nous sera utile plus tard.

Corollaire 4.9 - Soient $k$ un corps et $\Lambda$ une $\mathbb{Z} / N \mathbb{Z}$-algèbre avec $N \in \mathbb{N}$ un entier inversible dans $k$. On suppose que la p-dimension cohomologique de $k$ est finie pour tout nombre premier divisant $N$. Soit $K$ un complexe de préfaisceaux sur $\mathrm{Et} / k$. Alors, pour tout $r \in \mathbb{N}$, il existe un isomorphisme canonique $\iota_{k}^{*}(K(r))[2 r] \simeq \operatorname{REv}_{r}\left(L_{i}^{*}(K)\right)$ dans $\mathbf{D A}^{\text {eff, ét }}(k, \Lambda)$.

Demonstration En effet, $\mathrm{L} \hat{\imath}_{k}^{*}(K)$ est un antécédant (à isomorphisme près) de $K$ par l'équivalence $\mathrm{R} \hat{\imath}_{k_{*} *}$. Un tel antécédant à été construit dans l'étape $\mathrm{C}$ de la preuve du Lemme 4.6. Le résultat recherché est une conséquence immédiate de cette construction.

La preuve du résultat ci-dessous repose sur le Lemme 4.6.

Proposition 4.10 - Soient $S$ un schéma et $\Lambda$ une $\mathbb{Z} / N \mathbb{Z}$-algèbre avec $N \in \mathbb{N}$ un entier inversible dans $\mathcal{O}_{S}$. Il existe un morphisme canonique $\alpha_{S}: T_{S} \longrightarrow \iota_{S}^{*}(\Lambda(1))[2]$ dans $\mathbf{D A}^{\text {eff,ét }}(S, \Lambda)$. Il est donné par la composition de

$$
\left(\mathbb{P}_{S}^{1}, \infty_{S}\right) \otimes \Lambda \simeq\left(\mathbb{A}_{S}^{1}-0_{S}, 1_{S}\right) \otimes \Lambda[1] \longrightarrow \mathcal{O}^{\times} \otimes_{\mathbb{Z}} \Lambda[1] \simeq \iota_{S}^{*}(\Lambda(1))[2],
$$

où la flèche du milieu envoie un $X$-point $f: X \longrightarrow \mathbb{A}_{S}^{1}-0_{S}=S\left[t, t^{-1}\right]$, pour $X \in \mathrm{Sm} / S$, sur la fonction inversible $t \circ f \in \mathcal{O}^{\times}(X)$ et le dernier isomorphisme est déduit de la suite exacte courte de faisceaux étales

$$
0 \longrightarrow \iota_{S}^{*}\left(\mu_{n}\right) \longrightarrow \mathcal{O}^{\times} \stackrel{(-)^{n}}{\longrightarrow} \mathcal{O}^{\times} \longrightarrow 0
$$

De plus, le morphisme

$$
\operatorname{Sus}_{T, \Sigma}^{0}\left(T_{S}\right) \longrightarrow \mathrm{L} \hat{\imath}_{S}^{*}(\Lambda(1))[2],
$$

obtenu en appliquant $\operatorname{LSus}{ }_{T, \Sigma}^{0} \grave{a} \alpha_{S}$, est un isomorphisme dans $\mathbf{D A}^{\mathrm{e} t}(S, \Lambda)$.

Demonstration Seule la dernière assertion nécessite une preuve. Remarquons d'abord que $\alpha_{S}$ est compatible au changement de base, i.e., si $g: T \longrightarrow S$ est un morphisme de schémas, alors $g^{*}\left(\alpha_{S}\right)=\alpha_{T}$. Il suffit donc de prouver la proposition pour $S=\operatorname{Spec}(\mathbb{Z}[1 / N])$. En utilisant la Proposition 3.24, on se ramène au cas où $S=\operatorname{Spec}(k)$, avec $k$ un corps de $p$-dimension cohomologique finie pour tout nombre premier $p$ divisant $N$. (Si $N$ est pair, il faut également utiliser le Lemme 3.4 afin de remplacer $\mathbb{Q}$ par une extension totalement imaginaire.)

Encore une fois, nous pouvons travailler avec les $T$-spectres non symétriques (cf. le début de la preuve du Lemme 4.6) et c'est ce que nous ferons. L'endofoncteur hom $\left(\left(\mathbb{P}_{k}^{1}, \infty\right),-\right)$ de la catégorie $\operatorname{Spt}_{T}(\mathbf{C p l}(\mathbf{P S h}(\operatorname{Sm} / k, \Lambda)))$ est un équivalence de Quillen à droite relativement à la structure $\left(\mathbb{A}^{1}\right.$, ét)-locale stable (cf. [5, Th. 4.3.38]). Il suffit donc de prouver que le morphisme

$$
\operatorname{Rhom}\left(\left(\mathbb{P}_{k}^{1}, \infty\right), \operatorname{Sus}_{T}^{0}\left(T_{k}\right)\right) \longrightarrow \operatorname{Rhom}\left(\left(\mathbb{P}_{k}^{1}, \infty\right), \operatorname{L} \hat{\imath}_{k}^{*}(\Lambda(1))[2]\right)
$$


est un isomorphisme dans $\mathbf{D} \mathbf{A}^{\text {ét }}(k, \Lambda)$. Par ailleurs, le morphisme canonique

$$
\operatorname{Sus}_{T}^{0}\left(\Lambda_{c s t}\right) \longrightarrow \operatorname{Rhom}\left(\left(\mathbb{P}_{k}^{1}, \infty\right), \operatorname{Sus}_{T}^{0}\left(T_{k}\right)\right),
$$

avec $\Lambda_{c s t}=\iota_{k}^{*}(\Lambda)$ le préfaisceau constant de valeur $\Lambda$, est inversible dans $\mathbf{D A}^{\text {ét }}(k, \Lambda)$. Il suffit donc de prouver que le morphisme

$$
\mathrm{L}_{k}^{*}(\Lambda(0)) \simeq \operatorname{Sus}_{T}^{0}\left(\Lambda_{c s t}\right) \longrightarrow \operatorname{Rhom}\left(\left(\mathbb{P}_{k}^{1}, \infty\right), \operatorname{L} \hat{\imath}_{k}^{*}(\Lambda(1))[2]\right),
$$

donné au niveau zéro par le morphisme $\alpha_{k}^{\prime}: \Lambda_{c s t} \longrightarrow \underline{\operatorname{RHom}}\left(T_{k}, \iota_{k}^{*}(\Lambda(1))\right.$ [2]) qui envoie 1 sur $\alpha_{k}$, est inversible dans $\mathbf{D A}^{\text {ét }}(k, \Lambda)$. Or, le calcul de la cohomologie étale de la droite projective montre que le morphisme $\alpha_{k}^{\prime}$ est un isomorphisme dans $\mathbf{D A}{ }^{\text {eff,ét }}(k, \Lambda)$. En utilisant le Corollaire 4.9, on déduit aussitôt que le morphisme (40) devient un isomorphisme lorsqu' on lui applique $\mathrm{REv}_{0}$. Il est donc lui-même un isomorphisme puisque $\mathrm{R} \hat{\iota}_{k *}=\mathrm{R} \iota_{k_{*}} \circ \mathrm{REv_{0 }}$ est une équivalence de catégories par le Lemme 4.6.

Corollaire 4.11 - Avec les notations et sous les hypothèses $d u$ Théorème 4.1, le morphisme d'unité id $\longrightarrow \mathrm{L} \hat{\imath}_{S *} \circ \mathrm{R} \hat{\imath}_{S}^{*}$ est inversible.

Demonstration Comme dans la preuve du Lemme 4.6, nous pouvons travailler avec les $T$-spectres non symétriques. (On utilise le Lemme 4.2 pour s'assurer que $\mathrm{RHom}\left(T_{S},-\right.$ ) commute aux compositions transfinies.) Soit $K$ un complexe de préfaisceaux sur Et $/ S$ que nous supposerons projectivement cofibrant. D'après [5, Th. 4.3.61], il s'agit de montrer que le morphisme évident

$$
\iota_{S}^{*} K \longrightarrow \underset{n \in \mathbb{N}}{\operatorname{hocolim}} \underline{\operatorname{Rom}}\left(T_{S}^{\otimes n}, T_{S}^{\otimes n} \otimes \iota_{S}^{*} K\right)
$$

est inversible dans $\mathbf{D} \mathbf{A}^{\text {eff, ét }}(S, \Lambda)$. Vu le Lemme 4.2, il revient au même de montrer que le morphisme évident

$$
\iota_{S}^{*} K \longrightarrow \underset{n \in \mathbb{N}}{\operatorname{hocolim}} \operatorname{RHom}\left(T_{S}^{\otimes n}, \underset{m \in \mathbb{N}}{\operatorname{hocolim}} \operatorname{R} \underline{\operatorname{Hom}}\left(T_{S}^{\otimes m}, T_{S}^{\otimes m+n} \otimes \iota_{S}^{*} K\right)\right)
$$

est inversible dans $\mathbf{D} \mathbf{A}^{\text {eff, ét }}(S, \Lambda)$. D'après la Proposition 4.10, on dispose d'un morphisme

$$
\alpha_{S}^{n}: T_{S}^{\otimes n} \otimes \iota_{S}^{*} K \longrightarrow \iota_{S}^{*}(\Lambda(n))[2 n] \otimes \iota_{S}^{*}(K) \simeq \iota_{S}^{*}(K(n))[2 n]
$$

qui devient un isomorphisme dans $\mathbf{D A}^{\text {ét }}(S, \Lambda)$ après l'application du foncteur $\operatorname{Sus}_{T}^{0}$. Ceci entraîne, par une deuxième application de [5, Th. 4.3.61], que le morphisme

$$
\underset{m \in \mathbb{N}}{\operatorname{hocolim}} \operatorname{R} \underline{\operatorname{Hom}}\left(T_{S}^{\otimes m}, T_{S}^{\otimes m+n} \otimes \iota_{S}^{*} K\right) \stackrel{\alpha_{S}^{n}}{\longrightarrow} \underset{m \in \mathbb{N}}{\operatorname{hocolim}} \operatorname{RHom}\left(T_{S}^{\otimes m}, T_{S}^{\otimes m} \otimes \iota_{S}^{*}(K(n))[2 n]\right)
$$

est inversible dans $\mathbf{D} \mathbf{A}^{\text {eff, ét }}(S, \Lambda)$. Il suffit donc de prouver que le morphisme

$$
\iota_{S}^{*} K \longrightarrow \underset{n \in \mathbb{N}}{\operatorname{hocolim}} \operatorname{RHom}\left(T_{S}^{\otimes n}, \underset{m \in \mathbb{N}}{\operatorname{hocolim}} \operatorname{RHom}\left(T_{S}^{\otimes m}, T_{S}^{\otimes m} \otimes \iota_{S}^{*}(K(n))[2 n]\right)\right),
$$

obtenu en composant (42) avec le morphisme déduit des $\alpha_{S}^{n}$, est inversible dans $\mathbf{D A}^{\text {eff, ét }}(S, \Lambda)$. En commutant les foncteurs $\mathrm{RHom}\left(T_{S}^{\otimes-},-\right)$ avec les colimites homotopiques de $\mathbb{N}$-suites, ce qui est justifié par le Lemme 4.2, on peut réécrire le morphisme (43) de la manière suivante :

$$
\iota_{S}^{*} K \longrightarrow \underset{m \in \mathbb{N}}{\operatorname{hocolim}} \underline{\operatorname{Rom}}\left(T_{S}^{\otimes m}, \underset{n \in \mathbb{N}}{\operatorname{hocolim}} \underline{\operatorname{Rom}}\left(T_{S}^{\otimes n}, T_{S}^{\otimes m} \otimes \iota_{S}^{*}(K(n))[2 n]\right)\right) .
$$

Pour tout $n \in \mathbb{N}$, on choisit une équivalence ét-locale $\iota_{S}^{*}(K(n))[2 n] \longrightarrow \mathbf{L}_{n}$ avec $\mathbf{L}_{n}$ un complexe de préfaisceaux projectivement ét-fibrant sur $\mathrm{Sm} / S$. Alors, d'après le Sous-lemme $4.7, \mathbf{L}_{n}$ est projectivement $\left(\mathbb{A}^{1}\right.$, ét)-fibrant. Puisque $\iota_{S}^{*}(K(n))[2 n]$ est projectivement cofibrant, on peut aussi supposer que $\mathbf{L}_{n}$ est projectivement cofibrant. Par ailleurs, dans $\mathbf{D A}^{\text {eff, ét }}(S, \Lambda)$, on a un isomorphisme

$$
\iota_{S}^{*}(K(n))[2 n] \simeq \mathrm{R} \underline{\operatorname{Hom}}\left(T_{S}, \iota_{S}^{*}(K(n+1))[2 n+2]\right) .
$$

Il existe alors un quasi-isomorphisme de complexes de préfaisceaux $\mathbf{L}_{n} \longrightarrow \underline{\operatorname{Hom}}\left(T_{S}, \mathbf{L}_{n+1}\right)$ qui réalise cet isomorphisme. Par adjonction, on déduit des morphismes $\gamma_{n}: T_{S} \otimes \mathbf{L}_{n} \longrightarrow \mathbf{L}_{n+1}$ et 
donc un $T$-spectre (non symétrique) $\mathbf{L}=\left(\mathbf{L}_{n}, \gamma_{n}\right)_{n \in \mathbb{N}}$ qui est projectivement stablement ( $\mathbb{A}^{1}$, ét)fibrant. Considérons maintenant le morphisme de $T$-spectres non symétriques

$$
\alpha_{S}^{m}:\left(T_{S}^{\otimes m} \otimes \mathbf{L}_{n}\right)_{n \in \mathbb{N}} \longrightarrow\left(\iota_{S}^{*}(\Lambda(m))[2 m] \otimes \mathbf{L}_{n}\right)_{n \in \mathbb{N}} .
$$

Ce morphisme correspond dans $\mathbf{D A}^{\text {ét }}(S, \Lambda)$ à $\operatorname{Sus}_{T, \Sigma}^{0}\left(\alpha_{S}^{m}\right) \otimes \mathrm{id} \mathbf{L}$. Il y est donc inversible par la Proposition 4.10. Joignant ceci à [5, Th. 4.3.61], on obtient que la colimite homotopique suivant $n \in \mathbb{N}$ des morphismes

$$
\mathrm{R} \underline{\operatorname{Hom}}\left(T_{S}^{\otimes n}, T_{S}^{\otimes m} \otimes \iota_{S}^{*}(K(n)[2 n])\right) \stackrel{\alpha_{S}^{m}}{\longrightarrow} \operatorname{RHom}\left(T_{S}^{\otimes n}, \iota_{S}^{*}(K(m+n))[2(m+n)]\right)
$$

est un isomorphisme dans $\mathbf{D} \mathbf{A}^{\text {eff, ét }}(S, \Lambda)$. En injectant ceci dans (44), on se ramène à montrer que le morphisme

$$
\iota_{S}^{*} K \longrightarrow \underset{m \in \mathbb{N}}{\operatorname{hocolim}} \underline{\operatorname{Rom}}\left(T_{S}^{\otimes m}, \underset{n \in \mathbb{N}}{\operatorname{hocolim}} \operatorname{RHom}\left(T_{S}^{\otimes n}, \iota_{S}^{*}(K(m+n))[2(m+n)]\right)\right)
$$

est inversible dans $\mathbf{D} \mathbf{A}^{\text {eff, ét }}(S, \Lambda)$. Il est équivalent de montrer que

$$
\iota_{S}^{*} K \longrightarrow \underset{n \in \mathbb{N}}{\operatorname{hocolim}} \underline{\operatorname{Hom}}\left(T_{S}^{\otimes n}, \iota_{S}^{*}(K(n))[n]\right)
$$

est inversible dans $\mathbf{D} \mathbf{A}^{\text {eff, ét }}(S, \Lambda)$. Ceci est clair.

On continue avec deux lemmes faciles traitant de la commutation des foncteurs $L \hat{\imath}_{S}^{*}$ avec certaines opérations de Grothendieck.

LeMMe 4.12

(a) Soit $f: Y \longrightarrow X$ un morphisme de schémas. Il existe des isomorphismes canoniques

$$
\mathrm{L} f^{*} \circ \mathrm{L} \hat{\imath}_{X}^{*} \simeq \mathrm{L} \hat{\imath}_{Y}^{*} \circ \mathrm{L} f^{*} \quad \text { et } \quad \mathrm{R} f_{*} \circ \mathrm{R} \hat{\imath}_{Y *} \simeq \mathrm{R} \hat{\imath}_{X *} \circ \mathrm{R} f_{*}
$$

(Ci-dessus, $\mathrm{L} f^{*}$ et $\mathrm{R} f_{*}$ désignent les foncteurs « image inverse » et «image direct » pour les catégories $\mathbf{D}^{\mathrm{e}}(-, \Lambda)$ et $\mathbf{D} \mathbf{A}^{\mathrm{e} t}(-, \Lambda)$.)

(b) Soit $u: U \longrightarrow X$ un morphisme étale. Il existe alors des isomorphismes canoniques

$$
\mathrm{L} u_{\sharp} \circ \mathrm{L} \hat{\imath}_{U}^{*} \simeq \mathrm{L} \hat{\imath}_{X}^{*} \circ \mathrm{L} u_{\sharp} \quad \text { et } \quad \mathrm{L} u^{*} \circ \mathrm{R} \hat{\imath}_{X *} \simeq \mathrm{R} \hat{\imath}_{U *} \circ \mathrm{L} u^{*}
$$

(Ci-dessus, $\mathrm{L} u_{\sharp}$ désigne l'adjoint à gauche du foncteur $\mathrm{L} u^{*}$ pour les catégories $\mathbf{D}^{\text {ét }}(-, \Lambda)$ et DA $^{\text {ét }}(-, \Lambda)$.)

Demonstration Il suffit de construire les isomorphismes (47) et (48) au niveau des catégories de modèles, ce qui est immédiat.

Le résultat suivant est plus intéressant. Il repose d'une manière essentielle sur l'axiome de localité qui est satisfait dans $\mathbf{D} \mathbf{A}^{\text {ét }}(-, \Lambda)$ et $\mathbf{D}^{\text {ét }}(-, \Lambda)$.

Lemme 4.13 - Soient $s: Z \hookrightarrow X$ une immersion fermée. Alors, le morphisme canonique

$$
\mathrm{L} \hat{\imath}_{X}^{*} \circ \mathrm{R} s_{*} \longrightarrow \mathrm{R} s_{*} \circ \mathrm{L} \hat{\imath}_{Z}^{*}
$$

est inversible.

Demonstration Soit $j: U \hookrightarrow X$ l'immersion ouverte complémentaire à $s$. La paire $\left(\mathrm{L} j^{*}, \mathrm{~L} s^{*}\right)$ est conservative sur $\mathbf{D}^{\text {ét }}(X, \Lambda)$. Il suffit donc de prouver que (49) est inversible après application de $L j^{*}$ et $\mathrm{L} s^{*}$. Or, $\mathrm{L} j^{*} \mathrm{~L} \hat{\imath}_{X}^{*} \mathrm{R} s_{*} \simeq \hat{\imath}_{U}^{*} \mathrm{~L} j^{*} \mathrm{R} s_{*} \simeq 0$ et $\mathrm{L} j^{*} \mathrm{R} s_{*} \mathrm{~L} \hat{\imath}_{Z}^{*} \simeq 0$. D'autre part, $\mathrm{L} s^{*} \mathrm{~L} \hat{\imath}_{X}^{*} \mathrm{R} s_{*} \simeq \mathrm{L} \hat{\imath}_{Z}^{*} \mathrm{~L} s^{*} \mathrm{R} s_{*} \simeq$ $\mathrm{L} \hat{\imath}_{Z}^{*}$ et $\mathrm{L} s^{*} \mathrm{R} s_{*} \mathrm{~L} \hat{\imath}_{Z}^{*} \simeq \mathrm{L} \hat{\imath}_{Z}^{*}$. Le lemme est démontré.

On est maintenant en mesure d'achever la preuve du Théorème 4.1 .

Demonstration On suppose d'abord que $S$ est de dimension de Krull finie et de $p$-dimension cohomologique ponctuelle finie pour tout nombre premier $p$ divisant $N$. On montrera que le foncteur $L \hat{\imath}_{S}^{*}$ est une équivalence de catégories par induction noethérienne. Ainsi, on supposera que le résultat correspondant est vrai pour tout sous-schéma fermé strict de $S$. D'après le Corollaire 4.11 , le foncteur $\mathrm{L} \hat{\imath}_{S}^{*}$ est pleinement fidèle. Il suffit donc de voir que $\mathbf{D} \mathbf{A}^{\mathrm{e}}(S, \Lambda)$ est égale à sa plus 
petite sous-catégorie triangulée, notée provisoirement $\mathcal{T}$, stable par sommes infinies et contenant $\mathrm{L} \hat{\imath}_{S}^{*}\left(\mathbf{D}^{\text {ét }}(S, \Lambda)\right)$. Puisque $\mathbf{D} A^{\text {ét }}(S, \Lambda)$ est compactement engendrée (cf. la Proposition 3.19), il suffit de prouver que tout objet compact appartient à $\mathcal{T}$. On fixe donc $M$, un objet compact de $\mathbf{D A}^{\text {ét }}(S, \Lambda)$. Soit $\eta$ un point générique de $S$. D'après le Lemme 4.6, l'objet $\eta^{*} M \in \mathbf{D A}^{\text {ét }}(\kappa(\eta), \Lambda)$ appartient à l'image du foncteur $L \hat{\imath}_{\eta}^{*}$, qui est même une équivalence de catégories. On peut donc trouver un objet compact $K \in \mathbf{D}^{\text {ét }}(\kappa(\eta), \Lambda)$ tel que $\mathrm{L} \hat{\imath}_{\eta}^{*}(K) \simeq \eta^{*} M$. En utilisant la Remarque 3.23, on peut aussi trouver un objet compact $L \in \mathbf{D}^{\text {ét }}(S, \Lambda)$ tel que $\eta^{*}(L) \simeq K$. En utilisant le Lemme 4.12, on obtient en fin de compte un isomorphisme $\eta^{*}\left(L \hat{L}_{S}^{*} L\right) \simeq \eta^{*} M$. Par la Proposition 3.20, il existe alors une immersion ouverte $j: U \hookrightarrow S$ dont l'image contient $\eta$ et un isomorphisme $j^{*}\left(\mathrm{~L} \hat{\imath}_{S}^{*} L\right) \simeq j^{*} M$. On en déduit, à l'aide du Lemme 4.12, un triangle distingué

$$
\mathrm{L} \hat{\imath}_{S}^{*}\left(j_{\sharp} j^{*} L\right) \longrightarrow M \longrightarrow s_{*} s^{*} M \longrightarrow
$$

où $s: Z \hookrightarrow S$ est l'immersion fermée complémentaire à $j$. Or, par l'hypothèse de récurrence appliquée à $Z$, l'objet $s^{*} M$ est dans l'image de $\mathrm{L} \hat{\imath}_{Z}^{*}$, i.e., il existe $E \in \mathbf{D}^{\text {ét }}(Z, \Lambda)$ tel que $s^{*} M \simeq \mathrm{L} \hat{\imath}_{Z}^{*} E$. Du Lemme 4.13, on déduit que $s_{*} s^{*} M \simeq \mathrm{L} \hat{\imath}_{S}^{*}\left(s_{*} E\right)$. Ceci démontre le résultat recherché.

Il reste encore à nous débarasser de l'hypothèse de finitude des $p$-dimensions cohomologiques ponctuelles de $S$ pour ne retenir que la finitude des $p$-dimensions cohomologiques ponctuelles des hensélisés stricts de $S$. Vu le Corollaire 4.11, il s'agit de prouver que le morphisme de counité $\mathrm{L} \hat{\imath}_{S}^{*} \circ \mathrm{R} \hat{\iota}_{S *} \longrightarrow$ id est inversible. Cette question est locale pour la topologie étale sur $S$. (Utiliser pour cela le Lemme 4.12.) On peut donc supposer que $S$ est affine et que $\sqrt{-1} \in \mathcal{O}(S)$ si $N$ est pair. On raisonne ensuite comme au début, i.e., on utilise le Corollaire 4.11 pour se ramener à prouver que $\mathbf{D A}^{\text {ét }}(S, \Lambda)$ est égale à sa plus petite sous-catégorie triangulée stable par sommes infinies et contenant $\mathrm{L} \hat{\imath}_{S}^{*}\left(\mathbf{D}^{\text {ét }}(S, \Lambda)\right)$. D' après la Proposition 3.7, il suffit de prouver que $M=\operatorname{Sus}_{T, \Sigma}^{n}(X \otimes \Lambda)$ est dans $\operatorname{L} \hat{\imath}_{S}^{*}\left(\mathbf{D}^{\text {ét }}(S, \Lambda)\right)$ pour tout $n \in \mathbb{N}$ et $X \in \mathrm{Sm} / S$. On peut trouver un schéma affine $S_{0}$ de type fini sur sur $\mathbb{Z}[1 / N]$, et même sur $\mathbb{Z}[\sqrt{-1}, 1 / N]$ si $N$ est pair, et $X_{0} \in \operatorname{Sm} / S_{0}$ tels que $X \simeq S \times_{S_{0}} X_{0}$ pour un certain morphisme $q: S \longrightarrow S_{0}$. On a alors $M=q^{*} M_{0}$ avec $M_{0}=\operatorname{Sus}_{T, \Sigma}^{n}\left(X_{0} \otimes \Lambda\right)$. Puisque $S_{0}$ est de $p$-dimension cohomologique ponctuelle finie pour tout diviseur premier $p$ de $N$, on sait d'après ce qui précède que $M_{0} \simeq \mathrm{L} \hat{\imath}_{S_{0}}^{*} K_{0}$ pour un certain $K_{0} \in \mathbf{D}^{\text {ét }}\left(S_{0}, \Lambda\right)$. En utilisant le Lemme 4.12, on obtient en fin de compte que $M \simeq \mathrm{L} \hat{\imath}_{S}^{*}\left(q^{*} K_{0}\right)$. Ceci termine la preuve.

Le corollaire suivant est utile.

Corollaire 4.14 - Gardons les notations et les hypothèses du Théorème 4.1. Soit $\mathbf{F}$ un $T$ spectre symétrique en complexes de préfaisceaux de $\Lambda$-modules sur $\mathrm{Sm} / S$. Supposons que $\mathbf{F}$ est projectivement stablement ( $\mathbb{A}^{1}$, ét)-fibrant. Alors, pour tout $n \in \mathbb{N}$, le morphisme canonique $\iota_{S}^{*} \iota_{S *} \mathbf{F}_{n} \longrightarrow \mathbf{F}_{n}$ est une équivalence ét-locale et il existe un isomorphisme canonique $\iota_{S *} \mathbf{F}_{n} \simeq$ $\left(\iota_{S *} \mathbf{F}_{0}\right)(n)[2 n]$ dans $\mathbf{D}^{\mathrm{e} t}(S, \Lambda)$.

Demonstration Montrons d'abord que $\iota_{S}^{*} \iota_{S *} \mathbf{F}_{n} \longrightarrow \mathbf{F}_{n}$ est une équivalence ét-locale. Quitte à remplacer $\mathbf{F}$ par s ${ }_{-}^{n} \mathbf{F}$, on peut supposer que $n=0$. (Bien entendu, $\mathrm{s}_{-}$est le foncteur de décalage; voir [5, Déf. 4.3.13].) Soit $X$ un $S$-schéma lisse et notons $f: X \rightarrow S$ le morphisme structural. Il suffit de montrer que $\left(\iota_{S}^{*} \iota_{S *} \mathbf{F}_{0}\right)_{\mid \mathrm{Et} / X} \longrightarrow\left(\mathbf{F}_{0}\right)_{\mid \mathrm{Et} / X}$ est une équivalence ét-locale de préfaisceaux sur le petit site étale de $X$. Or, $\left(\iota_{S}^{*} \iota_{S *} \mathbf{F}_{0}\right)_{\mid \mathrm{Et} / X}$ s'identifie à $f^{*}\left(\iota_{S *} \mathbf{F}_{0}\right)=f^{*} \hat{\iota}_{S *} \mathbf{F}$ alors que $\left(\mathbf{F}_{0}\right)_{\mid \mathrm{Et} / X}$ s'identifie à $\iota_{X *} f^{*} \mathbf{F}_{0}=\hat{\iota}_{X *} f^{*} \mathbf{F}$. De plus, modulo ces identifications, le morphisme qui nous intéresse coïncide avec le morphisme $f^{*} \mathrm{R} \hat{\iota}_{S} \mathbf{F} \longrightarrow \mathrm{R} \hat{\imath}_{X *} f^{*} \mathbf{F}$ déduit de l'isomorphisme $\mathrm{L} f^{*} \mathrm{~L} \hat{\iota}_{S}^{*} \simeq \mathrm{L} \hat{\imath}_{X}^{*} \mathrm{~L} f^{*}$ du Lemme 4.12. Or, d'après le Théorème 4.1, $\mathrm{L} \hat{\imath}_{S}^{*}$ et $\mathrm{L} \hat{\imath}_{X}^{*}$ sont des équivalences de catégories et $\mathrm{R} \hat{\iota}_{S *}$ et $\mathrm{R} \hat{\iota}_{X *}$ sont leurs quasi-inverses. Le résultat recherché s'ensuit.

Pour démontrer la seconde assertion, on utilise que $\mathrm{s}_{-}^{n} \mathbf{F}$ est isomorphe à $\operatorname{Sus}_{T, \Sigma}^{0}\left(T^{n}\right) \otimes \mathbf{F}$ dans $\mathbf{D A}^{\text {ét }}(S, \Lambda)$. Il s'ensuit que $\iota_{S *} \mathbf{F}_{n}=\mathbf{R} \hat{\imath}_{S *} \mathrm{~s}_{-}^{n} \mathbf{F}$ est isomorphe à $\mathrm{R} \hat{\imath}_{S *}(\mathbf{F}(n)[2 n])$ qui à son tour est isomorphe à $\left(\operatorname{R} \hat{\iota}_{S *} \mathbf{F}\right)(n)[2 n]=\iota_{S *} \mathbf{F}_{0}(n)[2 n]$.

On a la conséquence suivante du Théorème 4.1.

Corollaire 4.15 - On garde les notations et les hypothèses du Théorème 4.1. Alors, le 2foncteur $\mathbf{D}^{\text {ét }}(-, \Lambda): \operatorname{Sch} / S \longrightarrow \mathfrak{I} \Re$, qui envoie un $S$-schéma quasi-projectif $X$ sur $\mathbf{D}^{\mathrm{e} t}(X, \Lambda)$ et 
un morphisme de $S$-schémas quasi-projectifs $f: Y \longrightarrow X$ sur $f^{*}: \mathbf{D}^{\text {ét }}(X, \Lambda) \longrightarrow \mathbf{D}^{\text {ét }}(Y, \Lambda)$, est un 2-foncteur homotopique stable. De plus, les foncteurs $\mathrm{L}_{-}^{*}: \mathbf{D}^{\text {ét }}(-, \Lambda) \longrightarrow \mathbf{D A}^{\text {ét }}(-, \Lambda)$ définissent une équivalence de 2-foncteurs homotopiques stables.

Remarque 4.16 - Le Corollaire 4.15, et plus précisément le fait que $\mathbf{D}^{\text {ét }}(-, \Lambda): \operatorname{Sch} / S \longrightarrow \mathfrak{I} \Re$ est un 2-foncteur homotopique stable, permet de retrouver une bonne partie des résultats classiques en cohomologie étale [3, Expo. XII, XIII, XVI, XVII et XVIII] par une voie partiellement nouvelle. Ainsi, la propriété que le foncteur $f^{*}: \mathbf{D}^{\text {ét }}(X, \Lambda) \longrightarrow \mathbf{D}^{\text {ét }}(Y, \Lambda)$ possède un adjoint à gauche lorsque $f$ est lisse fait partie de la définition d'un 2-foncteur homotopique stable. Il s'agit là d'une propriété profonde qui découle de [3, Expo. XVIII]. De même, le théorème de changement de base par un morphisme lisse est l'une des propriétés de base que doit vérifier un 2-foncteur homotopique stable. Par ailleurs, en appliquant les résultats de [4], on retrouve aussi le théorème de changement de base pour un morphisme projectif ainsi que le formalisme de dualité. De plus, ce n'est pas circulaire ! En effet, dans la preuve du Théorème 4.1 on utilise très peu de résultats de [3], à savoir : l'invariance par homotopie de la cohomologie étale et le calcul de $\mathrm{R} p_{*} p^{*}$ lorsque $p: \mathbb{P}_{X}^{1} \longrightarrow X$ est la projection de la droite projective relative.

\section{La réalisation étale des motifs étales}

Dans cette section, nous utiliserons le Théorème 4.1 pour construire la réalisation étale dont il est question dans cet article. On fixe un schéma de base $S$ et un anneau de coefficients $\Lambda$. On se donne aussi un idéal $J \subset \Lambda$. Vus les conditions d'application du Théorème 4.1, nous sommes amenés à introduire l'hypothèse suivante.

Нyротнѐse 5.1 - L'anneau $\Lambda / J$ est de caractéristique $N>0$ inversible dans $\mathcal{O}_{S}$. En tout point géométrique $\bar{x}$ de $S$ la $p$-dimension cohomologique ponctuelle de l'hensélisé strict de $S$ en $\bar{x}$ est finie si $p$ est un diviseur premier de $N$.

Le foncteur « changement de coefficients $»(-) \otimes_{\Lambda} \Lambda / J: \mathbf{C p l}(\Lambda) \longrightarrow \mathbf{C p l}(\Lambda / J)$ est de Quillen à gauche. Par la Proposition A.2, il induit un foncteur de Quillen à gauche sur les catégories de $T$ spectres symétriques en complexes de préfaisceaux sur $\mathrm{Sm} / S$. En le dérivant à gauche, on obtient un foncteur triangulé, monoïdal symétrique et unitaire

$$
(-) \otimes_{\Lambda} \Lambda / J: \mathbf{D A}^{\mathrm{ét}}(S, \Lambda) \longrightarrow \mathbf{D A}^{\mathrm{e} t}(S, \Lambda / J) .
$$

Definition 5.2 - Soient $S$ un schéma, $\Lambda$ un anneau et $J \subset \Lambda$ un idéal. On suppose que l'Hypothèse 5.1 est satisfaite. La réalisation étale $\Re_{S}^{\text {ét }}: \mathbf{D A}^{\mathrm{e} t}(S, \Lambda) \longrightarrow \mathbf{D}^{\text {ét }}(S, \Lambda / J)$ est la composition de

$$
\mathbf{D A}^{\text {ét }}(S, \Lambda) \stackrel{-\otimes_{\Lambda} \Lambda / J}{\longrightarrow} \mathbf{D A}^{\text {ét }}(S, \Lambda / J) \stackrel{\mathrm{R}_{\hat{\iota}_{*}}}{\sim} \mathbf{D}^{\text {ét }}(S, \Lambda / J) .
$$

C'est un foncteur triangulé, monoïdal symétrique et unitaire.

Nous voudrons aussi définir une réalisation étale à coefficients $J$-adic à la Ekedahl [13]. (Pour une introduction rapide à la catégorie d'Ekedahl, on renvoie le lecteur à [26, App. A.1] duquel nous empruntons quelques notations.) On note $\Lambda / J^{*}$ le diagramme d'anneaux indexé par $\mathbb{N}^{o p}$ :

$$
\left\{\cdots \rightarrow \Lambda / J^{s+1} \longrightarrow \Lambda / J^{s} \longrightarrow \cdots \longrightarrow \Lambda / J \longrightarrow 0\right\}
$$

On pensera à $\Lambda / J^{*}$ comme à un anneau dans le topos des préfaisceaux d'ensembles sur l'ensemble ordonné $(\mathbb{N}, \leq)$. En particulier, on peut parler de modules sur $\Lambda / J^{*}$; ils forment une catégorie abélienne de Grothendieck qu'on notera $\operatorname{Mod}\left(\Lambda / J^{*}\right)$. Concrètement, un $\Lambda / J^{*}$-module est un diagramme de $\Lambda$-modules $M=\left(M_{s}\right)_{s \in \mathbb{N}}$, indexé par $\mathbb{N}^{\text {op }}$ et tel que l'idéal $J^{s}$ est contenu dans l'annulateur de $M_{s}$ pour tout $s \in \mathbb{N}$. En particulier, chaque $M_{s}$ est naturellement un $\Lambda / J^{s}$-module et les morphismes $M_{s+1} \longrightarrow M_{s}$ sont $\Lambda / J^{s+1}$-linéaires.

Pour $s \in \mathbb{N}$, on dispose d'un foncteur évident $s^{*}: \operatorname{Mod}\left(\Lambda / J^{*}\right) \longrightarrow \operatorname{Mod}\left(\Lambda / J^{s}\right)$ qui envoie un $\Lambda / J^{*}$-module $M=\left(M_{s}\right)_{s \in \mathbb{N}}$ sur le $\Lambda / J^{s}$-module $M_{s}$. Ce foncteur possède un adjoint à gauche $s_{\sharp}$ et 
un adjoint à droite $s_{*}$. Ils envoient un $\Lambda / J^{s}$-module $Q$ sur les $\Lambda / J^{*}$-modules

$$
\begin{aligned}
& \left\{\cdots \longrightarrow 0 \longrightarrow \cdots \longrightarrow 0 \longrightarrow \stackrel{s}{Q} \longrightarrow Q / J^{s-1} Q \longrightarrow \cdots \longrightarrow Q / J Q \longrightarrow 0\right\} \\
& \text { et } \quad\{\cdots \stackrel{\text { id }}{\longrightarrow} Q \stackrel{\text { id }}{\longrightarrow} \cdots \stackrel{\text { id }}{\longrightarrow} Q \stackrel{\text { id }}{\longrightarrow} \stackrel{s}{Q} \longrightarrow 0 \longrightarrow \cdots \longrightarrow 0\}
\end{aligned}
$$

respectivement. On dispose par ailleurs d'un foncteur $(-) \otimes_{\Lambda} \Lambda / J^{*}: \operatorname{Mod}(\Lambda) \longrightarrow \operatorname{Mod}\left(\Lambda / J^{*}\right)$ qui à un $\Lambda$-module $M$ associe la suite $\left(M / J^{s} M\right)_{s \in \mathbb{N}}$. Son adjoint à droite est le foncteur $\lim _{s \in \mathbb{N}}$ qui envoie un $\Lambda / J^{*}$-module $\left(M_{s}\right)_{s \in \mathbb{N}}$ sur le $\Lambda$-module $\lim _{s \in \mathbb{N}} M_{s}$.

On note $\mathbf{C p l}\left(\Lambda / J^{*}\right)$ la catégorie des complexes de $\Lambda / J^{*}$-modules. Elle admet une structure de modèles, qualifiée de semi-projective : les équivalences faibles sont les quasi-isomorphismes et les cofibrations sont les morphismes qui induisent des cofibrations projectives dans $\mathbf{C p l}\left(\Lambda / J^{s}\right)$ en appliquant le foncteur $s^{*}$ pour tout $s \in \mathbb{N}$. Un morphisme $M=\left(M_{s}\right)_{s \in \mathbb{N}} \longrightarrow N=\left(N_{s}\right)_{s \in \mathbb{N}}$ dans $\operatorname{Cpl}\left(\Lambda / J^{*}\right)$ est une fibration semi-projective si et seulement si $M_{s} \longrightarrow M_{s-1} \times_{N_{s-1}} N_{s}$ est surjectif pour tout $s \in \mathbb{N}$ (avec la convention que $M_{-1}=N_{-1}=0$ ). Avec la structure semi-projective, le foncteur

$$
s^{*}: \mathbf{C p l}\left(\Lambda / J^{*}\right) \longrightarrow \mathbf{C p l}\left(\Lambda / J^{s}\right)
$$

est de Quillen à gauche et à droite pour tout $s \in \mathbb{N}$. De même, le foncteur

$$
(-) \otimes_{\Lambda} \Lambda / J^{*}: \mathbf{C p l}(\Lambda) \longrightarrow \operatorname{Cpl}\left(\Lambda / J^{*}\right)
$$

est de Quillen à gauche.

On considère maintenant la catégorie $\mathbf{D A}^{\text {ét }}\left(S, \Lambda / J^{*}\right)$. C'est la catégorie notée $\mathbb{S H}_{\mathfrak{M}}^{T}(\mathscr{F}, \mathcal{J})$ dans [5, Déf. 4.5.21] où on prend :

- $\mathfrak{M}=\mathbf{C p l}\left(\Lambda / J^{*}\right)$, la catégorie des complexes de $\Lambda / J^{*}$-modules ;

- J la catégorie à un objet et une flèche, et $\mathscr{F}$ le foncteur qui pointe $S$;

$-T=\left(\mathbb{P}_{S}^{1}, \infty_{S}\right) \otimes\left(\Lambda / J^{*}\right)$;

- et $\tau=$ ét la topologie étale (cf. le début du $§ 4.5$ de [5]).

Par la Proposition A.2, les foncteurs de Quillen (50) et (51) induisent des foncteurs de Quillen sur les catégories des $T$-spectres symétriques en complexes de préfaisceaux sur $\mathrm{Sm} / S$. En les dérivant, on déduit des foncteurs triangulés, monoïdaux symétriques et unitaires

$$
\begin{gathered}
s^{*}: \mathbf{D A}^{\text {ét }}\left(S, \Lambda / J^{*}\right) \longrightarrow \mathbf{D A}^{\text {ét }}\left(S, \Lambda / J^{s}\right) \text { pour } s \in \mathbb{N} \\
\text { et }(-) \otimes_{\Lambda} \Lambda / J^{*}: \mathbf{D A}^{\text {ét }}(S, \Lambda) \longrightarrow \mathbf{D A}^{\text {ét }}\left(S, \Lambda / J^{*}\right) .
\end{gathered}
$$

On a le lemme utile suivant.

LEMME 5.3 - Lorsque s parcourt l'ensemble des entiers naturels, les foncteurs (52) forment une famille conservative.

Demonstration Les foncteurs

$$
s^{*}: \operatorname{Spt}_{T}^{\Sigma}\left(\mathbf{C p l}\left(\mathbf{P S h}\left(\operatorname{Sm} / S, \Lambda / J^{*}\right)\right)\right) \longrightarrow \operatorname{Spt}_{T}^{\Sigma}\left(\mathbf{C p l}\left(\mathbf{P S h}\left(\operatorname{Sm} / S, \Lambda / J^{s}\right)\right)\right)
$$

sont de Quillen à gauche et à droite. De plus, ils préservent les quasi-isomorphismes de complexes de préfaisceaux niveau par niveau. On déduit aussitôt qu'ils préservent les équivalences ( $\mathbb{A}^{1}$, ét)locales stables et les objets stablement $\left(\mathbb{A}^{1}\right.$, ét)-fibrants.

Ceci dit, soit $f$ une flèche de $\mathbf{D A}^{\text {ét }}\left(S, \Lambda / J^{*}\right)$ et supposons que $s^{*}(f)$ est un isomorphisme pour tout $s \in \mathbb{N}$. On cherche à montrer que $f$ est un isomorphisme. On ne restreint pas la généralité en supposant que $f$ est la classe d'un morphisme $f: \mathbf{E} \longrightarrow \mathbf{F}$ entre $T$-spectres symétriques stablement $\left(\mathbb{A}^{1}\right.$, ét)-fibrants. Alors, $s^{*}(f): s^{*}(\mathbf{E}) \longrightarrow s^{*}(\mathbf{F})$ est une équivalence $\left(\mathbb{A}^{1}\right.$, ét)-locale stable entre $T$ spectres symétriques stablement ( $\mathbb{A}^{1}$, ét)-fibrants. C'est donc un quasi-isomorphisme de complexes de préfaisceaux niveau par niveau. Puisque que les foncteurs $s^{*}: \mathbf{D}\left(\Lambda / J^{*}\right) \longrightarrow \mathbf{D}\left(\Lambda / J^{s}\right)$, pour $s \in \mathbb{N}$, forment une famille conservative, on déduit que $f$ est aussi un quasi-isomorphisme de complexes de préfaisceaux niveau par niveau. Ceci termine la preuve du lemme. 
On considère ensuite la catégorie $\mathbf{D}^{\text {ét }}\left(S, \Lambda / J^{*}\right)$. C'est la catégorie homotopique, relativement à la structure ét-locale (cf. [5, Déf. 4.4.33]), de $\operatorname{Cpl}\left(\mathbf{P S h}\left(\mathrm{Et} / S, \Lambda / J^{*}\right)\right)$. Comme avant, on a des foncteurs

$$
s^{*}: \mathbf{D}^{\text {ét }}\left(S, \Lambda / J^{*}\right) \longrightarrow \mathbf{D}^{\text {ét }}\left(S, \Lambda / J^{s}\right)
$$

pour $s \in \mathbb{N}$. L'analogue du Lemme 5.3 est également vrai. Sa preuve est laissée au lecteur.

LEMME 5.4 - Lorsque s parcourt l'ensemble des entiers naturels, les foncteurs (54) forment une famille conservative.

Enfin, on a une adjonction de Quillen

$$
\left(\iota_{S}^{*}, \iota_{S *}\right): \mathbf{C p l}\left(\mathbf{P S h}\left(\mathrm{Et} / S, \Lambda / J^{*}\right)\right) \rightleftarrows \mathbf{C p l}\left(\mathbf{P S h}\left(\operatorname{Sm} / S, \Lambda / J^{*}\right)\right)
$$

et on pose $\hat{\iota}_{S}^{*}=\operatorname{Sus}_{T, \Sigma}^{0} \circ \iota_{S}^{*}$ et $\hat{\iota}_{S *}=\iota_{S *} \circ \operatorname{Ev}_{0}$. En dérivant l'adjonction de Quillen $\left(\hat{\iota}_{S}^{*}, \hat{\iota}_{S *}\right)$, on obtient l'adjonction

$$
\left(\mathrm{L} \hat{\imath}_{S}^{*}, \mathrm{R} \hat{\imath}_{S *}\right): \mathbf{D}^{\mathrm{e} t}\left(S, \Lambda / J^{*}\right) \rightleftarrows \mathbf{D A}^{\mathrm{e} t}\left(S, \Lambda / J^{*}\right)
$$

Theoreme 5.5 - Soient $S$ un schéma, $\Lambda$ un anneau et $J \subset \Lambda$ un idéal. On suppose que l'Hypothèse 5.1 est satisfaite. Alors, $\hat{L}_{S}^{*}: \mathbf{D}^{\mathrm{t} t}\left(S, \Lambda / J^{*}\right) \longrightarrow \mathbf{D A}^{\mathrm{t}}\left(S, \Lambda / J^{*}\right)$ est une équivalence de catégories.

Demonstration Il faut montrer que l'unité et la counité de l'adjonction ( $\left.\mathrm{L} \hat{\imath}_{S}^{*}, \mathrm{R} \hat{\imath}_{S *}\right)$ sont inversibles. D'après les Lemmes 5.3 et 5.4, il suffit de faire cela après applications des foncteurs $s^{*}$, pour $s \in \mathbb{N}$. Or, on dispose de deux carrés commutatifs

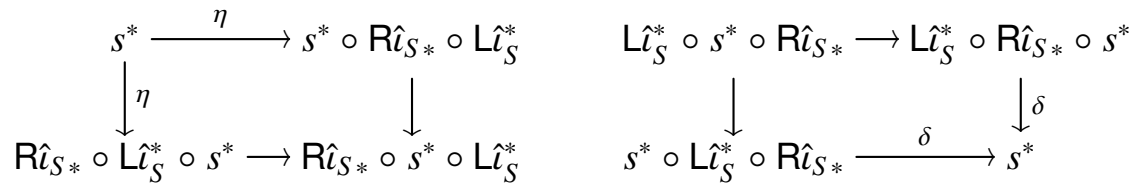

Par le Théorème 4.1, $\eta: s^{*} \longrightarrow \operatorname{R} \hat{\imath}_{S *} \circ \mathrm{L} \hat{\imath}_{S}^{*} \circ s^{*}$ et $\delta: \operatorname{L} \hat{\imath}_{S}^{*} \circ \mathrm{R} \hat{\imath}_{S *} \circ s^{*} \longrightarrow s^{*}$ sont inversibles. Il reste donc à montrer que les transformations naturelles $s^{*} \circ \mathrm{L} \hat{\imath}_{S}^{*} \longrightarrow \mathrm{L} \hat{\imath}_{S}^{*} \circ s^{*}$ et $s^{*} \circ \operatorname{R} \hat{\imath}_{S *} \longrightarrow \operatorname{R} \hat{\imath}_{S *} \circ s^{*}$ sont inversibles. Puisque les foncteurs $s^{*}$ sont de Quillen à gauche et à droite, il suffit de faire cela au niveau des catégories de modèles. Ceci est alors évident.

On arrive enfin à la construction de la réalisation étale $J$-adique.

Definition 5.6 - Soient $S$ un schéma, $\Lambda$ un anneau et $J \subset \Lambda$ un idéal. On suppose que $l$ 'Hypothèse 5.1 est satisfaite. La réalisation étale $\mathfrak{R}_{S}^{\text {ét }}: \mathbf{D A}^{\mathrm{e}}(S, \Lambda) \longrightarrow \mathbf{D}^{\mathrm{ét}}\left(S, \Lambda / J^{*}\right)$, est la composition de

$$
\mathbf{D A}^{\text {ét }}(S, \Lambda) \stackrel{-\otimes_{\Lambda} \Lambda / J^{*}}{\longrightarrow} \mathbf{D A}^{\text {ét }}\left(S, \Lambda / J^{*}\right) \stackrel{\mathrm{R} \hat{\imath}_{S_{*}}}{\sim} \mathbf{D}^{\text {ét }}\left(S, \Lambda / J^{*}\right) .
$$

C'est un foncteur triangulé, monö̈dal symétrique et unitaire.

Le foncteur de réalisation $J$-adic que l'on vient de définir se factorise par une sous-catégorie pleine de $\mathbf{D}^{\text {ét }}\left(S, \Lambda / J^{*}\right)$, la catégorie dérivée des faisceaux $J$-adiques d'Ekedahl qu'on introduit maintenant.

Definition 5.7 - Soient $S$ un schéma, $\Lambda$ un anneau et $J \subset \Lambda$ un idéal. On note $\hat{\mathbf{D}}^{\text {ét }}\left(S, \Lambda_{J}\right) \subset$ $\mathbf{D}^{\mathrm{t}}\left(S, \Lambda / J^{*}\right)$ la sous-catégorie pleine formée des complexes de préfaisceaux de $\Lambda / J^{*}$-modules $K$ tels que pour tout $s \in \mathbb{N}$, le morphisme canonique

$$
(s+1)^{*} K^{\mathrm{L}} \otimes_{\Lambda / J^{s+1}}\left(\Lambda / J^{s}\right) \longrightarrow s^{*} K
$$

est une équivalence ét-locale.

Proposition 5.8 - Soient $S$ un schéma, $\Lambda$ un anneau et $J \subset \Lambda$ un idéal. On suppose que l'Hypothèse 5.1 est satisfaite. Alors, l'image de $\Re_{S}^{\mathrm{ét}}: \mathbf{D A}^{\mathrm{e} t}(S, \Lambda) \longrightarrow \mathbf{D}^{\mathrm{ét}}\left(S, \Lambda / J^{*}\right)$ est contenue dans $\hat{\mathbf{D}}^{\text {ét }}\left(S, \Lambda_{J}\right)$. 
Demonstration On définit une sous-catégorie triangulée $\hat{\mathbf{D A}} \hat{A}^{\text {ét }}\left(S, \Lambda_{J}\right) \subset \mathbf{D A}^{\text {ét }}\left(S, \Lambda / J^{*}\right)$ en retenant les objets $A \in \mathbf{D A}^{\text {ét }}\left(X, \Lambda / J^{*}\right)$ tels que les morphismes

$$
(s+1)^{*} A \otimes_{\Lambda / J^{s+1}}\left(\Lambda / J^{s}\right) \longrightarrow s^{*} A
$$

sont inversibles pour tous les $s \in \mathbb{N}$. L'équivalence $L \iota_{S}^{*}: \mathbf{D}^{\text {ét }}\left(S, \Lambda / J^{*}\right) \simeq \mathbf{D A}^{\text {ét }}\left(S, \Lambda / J^{*}\right)$ induit alors une équivalence $\mathrm{L}_{S}^{*}: \hat{\mathbf{D}}^{\text {et }}\left(S, \Lambda_{J}\right) \simeq \hat{\mathbf{D A}} \hat{\mathrm{e}}^{\text {et }}\left(S, \Lambda_{J}\right)$. Il est donc suffisant de montrer que l'image du foncteur

$$
-\otimes_{\Lambda}\left(\Lambda / J^{*}\right): \mathbf{D A}^{\mathrm{ét}}(S, \Lambda) \longrightarrow \mathbf{D A}^{\mathrm{ét}}\left(S, \Lambda / J^{*}\right)
$$

est contenue dans $\hat{\mathbf{D A}}{ }^{\text {ét }}\left(S, \Lambda_{J}\right)$. Or, pour $E \in \mathbf{D A}^{\text {ét }}(S, \Lambda)$, on a $s^{*}\left(E \otimes_{\Lambda}\left(\Lambda / J^{*}\right)\right) \simeq E \otimes_{\Lambda}\left(\Lambda / J^{s}\right)$. On est donc ramené à montrer que les morphismes

$$
\left(E \otimes_{\Lambda}\left(\Lambda / J^{s+1}\right)\right) \otimes_{\Lambda / J^{s+1}}\left(\Lambda / J^{s}\right) \longrightarrow E \otimes_{\Lambda}\left(\Lambda / J^{s}\right)
$$

sont inversibles. Ceci est clair.

Dans [26], Ivorra construit une réalisation étale sur une catégorie de motifs géométriques à la Voevodsky. Nous allons expliquer le lien entre sa construction et la notre. Ceci ne sera pas utilisé dans la suite de l'article et le lecteur non intéressé peut ignorer le reste de cette section.

Rappelons brièvement la construction d'Ivorra. Soit $S$ un schéma de base. On note $\operatorname{Cor}(S)$ la catégorie ayant pour objets les $S$-schémas lisses et ayant pour morphismes les correspondances finies au-dessus de $S$. C'est une catégorie additive et on peut former la catégorie triangulée $\mathbf{K}^{b}(\mathbf{C o r}(S))$ des complexes bornés et des morphismes de complexes à homotopie près. La réalisation étale d'Ivorra est définie sur $\mathbf{D M}_{\mathrm{gm}}^{\mathrm{eff}}(S)$, l'enveloppe pseudo-abélienne du quotient de Verdier de $\mathbf{K}^{b}(\mathbf{C o r}(S))$ par sa sous-catégorie triangulée engendrée par les complexes de la forme :

- $\left[\mathbb{A}_{X}^{1} \longrightarrow X\right]$ pour $X \in \mathrm{Sm} / S$, et

- $[V \longrightarrow U \oplus Y \longrightarrow X]$ pour un carré Nisnevich de $S$-schémas lisses<smiles>[Al]1[Te][Te][Tl]1</smiles>

Pour la suite, on retient également la variante «sans transferts » de la catégorie $\mathbf{D} \mathbf{M}_{\mathrm{gm}}^{\mathrm{eff}}(S)$ qu'on obtient en remplaçant $\operatorname{Cor}(S) \operatorname{par}(\mathrm{Sm} / S) \otimes \mathbb{Z}$, la catégorie ayant pour objets les $S$-schémas lisses et ayant pour morphismes les combinaisons linéaires de $S$-morphismes. Cette variante « sans transferts » sera notée $\mathbf{D} \mathbf{A}_{\mathrm{gm}}^{\mathrm{eff}}(S)$.

Soit $\ell$ un nombre premier inversible sur $S$. Le point de départ de la construction d'Ivorra consiste à se donner un remplacement projectivement ét-fibrant du préfaisceau constant $\mathbb{Z} / \ell^{*}$ dans la catégorie $\mathbf{C p l}\left(\mathbf{P S T}\left(\mathrm{Sm} / S, \mathbb{Z} / \ell^{*}\right)\right)$ des préfaisceaux avec transferts en $\mathbb{Z} / \ell^{*}$-modules sur $\mathrm{Sm} / S$. En fait, beaucoup d'effort dans [26] est consacré à montrer qu'on peut remplacer $\mathbb{Z} / \ell^{*}$ par sa résolution de Godement; le point difficile étant de construire des transferts sur le complexe de Godement. Mais pour nous, il sera inutile d'avoir un remplacement particulier. Ainsi, on peut invoquer une structure de modèles ét-locale sur $\mathbf{C p l}\left(\mathbf{P S T}\left(\mathrm{Sm} / S, \mathbb{Z} / \ell^{*}\right)\right)$ pour obtenir une équivalence ét-locale de complexes de préfaisceaux avec transferts $\mathbb{Z} / \ell^{*} \mathbb{Z} \longrightarrow \mathcal{G}^{*}$ avec $\mathcal{G}^{*}$ ét-fibrant. En particulier, pour tout $X \in \mathrm{Sm} / S$ et $n \in \mathbb{Z}$, on a un isomorphisme canonique $\mathrm{H}_{\text {et }}^{n}\left(X, \mathbb{Z} / \ell^{*}\right) \simeq \mathrm{H}_{-n}\left(\mathcal{G}^{*}(X)\right)$.

En utilisant $\mathcal{G}^{*}$, on obtient maintenant un foncteur $\operatorname{Cor}(S)^{\mathrm{op}} \longrightarrow \mathbf{C p l}\left(\mathbf{P S h}\left(\mathrm{Et} / S, \mathbb{Z} / \ell^{*}\right)\right)$ qui envoie $X \in \operatorname{Sm} / S$ sur $\left(\pi_{X}\right)_{*}\left(\mathcal{G}_{\mid \mathrm{Et} / X}^{*}\right)$ avec $\pi_{X}: X \longrightarrow S$ le morphisme structural. C'est un foncteur additif. Il se prolonge donc en un foncteur triangulé $\mathbf{K}^{b}(\operatorname{Cor}(S))^{\text {op }} \longrightarrow \mathbf{D}^{\text {ét }}\left(S, \mathbb{Z} / \ell^{*}\right)$. Il est facile de voir que ce foncteur passe au quotient de Verdier et à la pseudo-abélianisation pour fournir le foncteur de réalisation étale d'Ivorra

$$
\mathrm{R}_{S}^{\mathrm{Iv}}: \mathbf{D M}_{\mathrm{gm}}^{\mathrm{eff}}(S)^{\mathrm{op}} \longrightarrow \mathbf{D}^{\mathrm{e} \text { t }}\left(S, \mathbb{Z} / \ell^{*}\right)
$$

Il est clair que le foncteur $\mathrm{R}_{S}^{\mathrm{Iv}}$ est indépendant du choix de $\mathcal{G}^{*}$ à un isomorphisme canonique près. On peut maintenant énoncer la proposition suivante qui décrit le lien avec la réalisation étale de la Définition 5.6. 
Proposition 5.9 - Soient $S$ un schéma de base et $\ell$ un nombre premier inversible sur $S$. On suppose qu'en tout point géométrique $\bar{x}$ de $S$ la $\ell$-dimension cohomologique ponctuelle de l'hensélisé strict de $S$ en $\bar{x}$ est finie. Alors, on a un diagramme commutatif à un isomorphisme canonique près

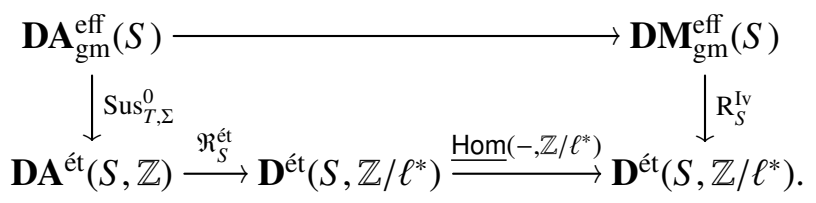

Demonstration Nous donnerons seulement une esquisse de la preuve. Remarquons d'abord que la restriction de $\mathrm{R}_{S}^{\mathrm{Iv}}$ à $\mathbf{D} \mathbf{A}_{\mathrm{gm}}^{\mathrm{eff}}(S)$, que l'on notera encore $\mathrm{R}_{S}^{\mathrm{Iv}}$, admet une description similaire à celle de (55). Plus précisément, on part d'un remplacement ét-fibrant $\mathcal{G}^{*}$ de $\mathbb{Z} / \ell^{*}$ dans la catégorie $\operatorname{Cpl}\left(\mathbf{P S h}\left(\operatorname{Sm} / S, \mathbb{Z} / \ell^{*}\right)\right)$. (Cette fois-ci on ne suppose pas que $\mathcal{G}^{*}$ possède des transferts.) On a alors un foncteur additif $(\mathrm{Sm} / S) \otimes \mathbb{Z} \longrightarrow \mathbf{C p l}\left(\mathbf{P S h}\left(\mathrm{Et} / S, \mathbb{Z} / \ell^{*}\right)\right)$ qui envoie $X \otimes \mathbb{Z} \operatorname{sur}\left(\pi_{X}\right)_{*}\left(\mathcal{G}_{\mid \mathrm{Et} / X}^{*}\right)$. Ce foncteur se prolonge aux complexes bornés, et passe au quotient de Verdier et à la pseudoabélianisation pour fournir le foncteur

$$
\mathrm{R}_{S}^{\mathrm{Iv}}: \mathbf{D A}_{\mathrm{gm}}^{\mathrm{eff}}(S)^{\mathrm{op}} \longrightarrow \mathbf{D}^{\text {ét }}\left(S, \mathbb{Z} / \ell^{*}\right)
$$

On se donne maintenant un remplacement projectivement stablement $\left(\mathbb{A}^{1}\right.$, ét)-fibrant $\mathbf{G}^{*} \mathrm{du}$ $T$-spectre symétrique $\operatorname{Sus}_{T, \Sigma}^{0}\left(\mathbb{Z} / \ell^{*}\right)$ dans $\operatorname{Spt}_{T}^{\Sigma}\left(\mathbf{C} \mathbf{p l}\left(\mathbf{P S h}\left(\operatorname{Sm} / S, \mathbb{Z} / \ell^{*}\right)\right)\right)$. On prendra dans la suite $\mathcal{G}^{*}=\mathbf{G}_{0}^{*}$. (Pour voir que $\mathbb{Z} / \ell^{*} \longrightarrow \mathbf{G}_{0}^{*}$ est une équivalence ét-locale, on utilise la généralisation du Corollaire 4.9 au cas d'un schéma de base $S$ qui découle aussitôt du Théorème 4.1.)

Soit $K$ un complexe borné dans $(\mathrm{Sm} / S) \otimes \mathbb{Z}$ que l'on considère comme un complexe de préfaisceaux sur $\mathrm{Sm} / S$ de la manière évidente. En revenant aux définitions, on voit que

$$
\underline{\operatorname{Hom}}\left(\Re_{S}^{\text {ét }}\left(\operatorname{Sus}_{T, \Sigma}^{0}(K)\right), \mathbb{Z} / \ell^{*}\right) \simeq \underline{\operatorname{Hom}}\left(\operatorname{R}_{S *} \operatorname{Sus}_{T, \Sigma}^{0}\left(K \otimes \mathbb{Z} / \ell^{*}\right), \operatorname{R} \hat{\iota}_{S *} \mathbf{G}^{*}\right) .
$$

Puisque R $\hat{\imath}_{S *}$ est une équivalence de catégories monoïdales fermées, le second membre de l'isomorphisme (57) s'identifie canoniquement à

$$
\mathrm{R} \hat{\iota}_{S} * \underline{\operatorname{Hom}}\left(\operatorname{Sus}_{T, \Sigma}^{0}\left(K \otimes \mathbb{Z} / \ell^{*}\right), \mathbf{G}^{*}\right)=\mathrm{R}_{S *} \underline{\operatorname{Hom}}\left(K \otimes \mathbb{Z} / \ell^{*}, \mathcal{G}^{*}\right)=\mathrm{R}^{\mathrm{Iv}}(K) .
$$

On obtient de cette manière un zigzag fonctoriel (en $K$ ) d'équivalences ét-locales entre les complexes $\underline{\operatorname{Hom}}\left(\Re_{S}^{\text {ét }}\left(\operatorname{Sus}_{T, \Sigma}^{0}(K)\right), \mathbb{Z} / \ell^{*}\right)$ et $\mathrm{R}^{\mathrm{Iv}}(K)$. Ceci termine notre esquisse de preuve.

\section{Compatibilité avec les six opérations}

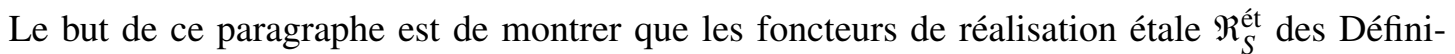
tions 5.2 et 5.6 commutent aux six opérations de Grothendieck. Nous commençons par traiter le problème analogue pour les foncteurs « changement de coefficients ».

On fixe un schéma de base $S$ et un anneau de coefficients $\Lambda$. On se donne aussi un morphisme d'anneaux $a: \Lambda \longrightarrow \Lambda^{\prime}$. On note $a^{*}: \mathbf{D A}^{\text {ét }}(-, \Lambda) \longrightarrow \mathbf{D A}^{\text {ét }}\left(-, \Lambda^{\prime}\right)$ les foncteurs déduits du foncteur de Quillen à gauche $-\otimes_{\Lambda} \Lambda^{\prime}: \mathbf{C p l}(\Lambda) \longrightarrow \mathbf{C p l}\left(\Lambda^{\prime}\right)$. (Voir le début de l'Annexe $\mathrm{A}$ et plus particulièrement (146).) Leurs adjoints à droite seront désignés par $a_{*}$. On a le résultat suivant.

Lemme 6.1 - Les foncteurs $a^{*}: \mathbf{D A}^{\mathrm{e} t}(-, \Lambda) \longrightarrow \mathbf{D A}^{\text {ét }}\left(-, \Lambda^{\prime}\right)$ définissent un morphisme de 2foncteurs homotopiques stables au-dessus de $S$ au sens de [7, Déf. 3.1].

Demonstration Ceci est le contenu du Lemme A.3.

Si $f$ est un morphisme de schémas, on note $\theta_{f}: f^{*} \circ a^{*} \stackrel{\sim}{\longrightarrow} a^{*} \circ f^{*} l^{\prime}$ 'isomorphisme canonique. Proposition 6.2 - Pour tout morphisme de $S$-schémas quasi-projectifs $f: X^{\prime} \longrightarrow X$, il existe des transformations naturelles canoniques

$$
\gamma_{f}: a^{*} \circ f_{*} \longrightarrow f_{*} \circ a^{*}, \quad \rho_{f}: f_{!} \circ a^{*} \longrightarrow a^{*} \circ f_{!} \quad \text { et } \xi_{f}: a^{*} \circ f^{!} \longrightarrow f^{!} \circ a^{*}
$$

compatibles à la composition des $S$-morphismes de schémas, et telles que

(a) $\gamma_{f}$ est inversible pour $f$ projectif; 
(b) $\rho_{f}$ est inversible sans hypothèse sur $f$;

(c) $\xi_{f}$ est inversible pour $f$ lisse.

Demonstration Il s'agit de [7, Th. 3.4] appliqué au morphisme de 2-foncteurs homotopiques stables du Lemme 6.1.

Theoreme 6.3 - On suppose que $S$ et $\Lambda$ satisfont à l'Hypothèse 3.17. Alors, les transformations naturelles $\gamma_{f}$ et $\xi_{f}$ de la Proposition 6.2 sont inversibles.

Demonstration Vue la Proposition 6.2, il suffit de montrer que $\gamma_{j}$ et $\xi_{i}$ sont inversibles lorsque $j$ est une immersion ouverte et $i$ est une immersion fermée. Si $i$ et $j$ sont complémentaires, le 2-triangle distingué de localité [4, Prop. 1.4.9] montre que ces deux propriétés sont équivalentes. (On utilise pour cela que $i_{*}$ est conservatif, que $j^{*}$ est essentiellement surjectif, et que les foncteurs $a^{*}$ commutent aux opérations $i_{*}$ et $j^{*}$.) Il suffit donc de traiter le cas de $\gamma_{j}$.

Dans la suite, on fixe un morphisme quasi-projectif $f$ et on montre que $\gamma_{f}$ est inversible. D'après le Lemme A.6, les foncteurs $a_{*}$ sont conservatifs. Il suffit donc de montrer que $\gamma_{f}$ : $a_{*} a^{*} f_{*} \longrightarrow a_{*} f_{*} a^{*}$ est inversible. Vu l'isomorphisme naturel $a_{*} f_{*} \simeq f_{*} a_{*}$, on est ramené à montrer que la transformation naturelle $a_{*} a^{*} f_{*} \longrightarrow f_{*} a_{*} a^{*}$ est inversible. Or, l'endofoncteur $a_{*} a^{*}$ de $\mathbf{D A}{ }^{\text {ét }}(-, \Lambda)$ est donné par $-\otimes_{\Lambda} \Lambda^{\prime}$ où $\Lambda^{\prime}$ est considéré comme un $\Lambda$-module. Il suffit donc de prouver que $f_{*}(-) \otimes_{\Lambda} M \longrightarrow f_{*}\left(-\otimes_{\Lambda} M\right)$ est inversible pour tout $M \in \mathbf{D}(\Lambda)$. Or, le foncteur $f_{*}$ commute aux sommes infinies d'après [4, Lem. 2.1.159] et la Proposition 3.19. Puisque la catégorie triangulée $\mathbf{D}(\Lambda)$ est compactement engendrée par $\Lambda$, il suffit de traiter le cas $M=\Lambda$. Le résultat recherché est maintenant clair.

Les foncteurs $a^{*}: \mathbf{D A}^{\text {ét }}(-, \Lambda) \longrightarrow \mathbf{D A}^{\text {ét }}\left(-, \Lambda^{\prime}\right)$ sont monoïdaux, symétriques et unitaires. Autrement dit, le morphisme de 2-foncteurs homotopiques stables du Lemme 6.1 est monoïdal symétrique et unitaire au sens de [7, Déf. 3.2].

Theoreme 6.4 - Soit X un $S$-schéma quasi-projectif. Il existe une transformation binaturelle en $A, B \in \mathbf{D A}^{\mathrm{e}} \mathrm{et}(X, \Lambda)$ :

$$
a^{*} \underline{\operatorname{Hom}}(A, B) \longrightarrow \underline{\operatorname{Hom}}\left(a^{*}(A), a^{*}(B)\right) .
$$

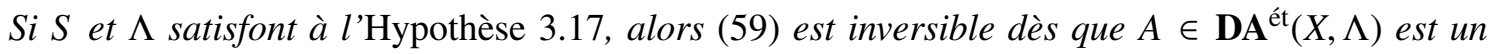
objet compact.

Demonstration La transformation binaturelle (59) est un cas particulier de [7, (3.1)]. On passe à la seconde partie de l'énoncé. D'après la Proposition 3.19 , la catégorie $\mathbf{D A} \mathbf{A}^{\text {ét }}(X, \Lambda)$ est compactement engendrée par les objets de la forme $f_{\sharp} \Lambda(n)$ avec $f: U \longrightarrow X$ un $X$-schéma lisse et $n \in \mathbb{Z}$. On peut donc supposer que $A=f_{\sharp} \Lambda(n)$. Par ailleurs, on dispose d'un isomorphisme binaturel $\underline{\operatorname{Hom}}\left(f_{\sharp}(-),-\right) \simeq f_{*} \operatorname{Hom}\left(-, f^{*}(-)\right)$. Le Théorème 6.3 et [7, Lem. 3.18] entraînent aussitôt qu'il suffit de prouver que $a^{*} \mathrm{Hom}\left(\Lambda(n), f^{*} B\right) \longrightarrow \underline{\operatorname{Hom}}\left(a^{*}(\Lambda(n)), a^{*} f^{*}(B)\right)$ est inversible. Or, la source et le but de cette flèche sont canoniquement isomorphes à $a^{*} f^{*}(B)(-n)$. Ceci termine la preuve du théorème.

Étant donnés un schéma $S$, un anneau $\Lambda$ et un idéal $J \subset \Lambda$, on introduit l'hypothèse suivante qui est équivalente à la conjonction des Hypothèses 3.17 et 5.1.

НуротнЀSE 6.5 — La dimension de Krull de $S$ est finie et les $p$-dimensions cohomologiques ponctuelles de $S$ sont uniformément bornées lorsque $p$ parcourt les nombres premiers non inversibles dans $\Lambda$. L'anneau $\Lambda / J$ est de caractéristique $N>0$ inversible dans $\mathcal{O}_{S}$.

Theoreme 6.6 - Soient $S$ un schéma de base, $\Lambda$ un anneau de coefficients et $J \subset \Lambda$ un idéal. On suppose que l'Hypothèse 6.5 est satisfaite. Alors, les foncteurs de réalisation étale $\Re^{\text {ét }}: \mathbf{D A}^{\mathrm{e} t}(-, \Lambda) \longrightarrow \mathbf{D}^{\text {ét }}(-, \Lambda / J)$ commutent aux six opérations de Grothendieck. Plus précisément, on a les propriétés suivantes.

(A) Pour tout morphisme de $S$-schémas quasi-projectifs $f: Y \longrightarrow X$, on a des isomorphismes naturels canoniques

$$
\theta_{f}: f^{*} \circ \Re_{X}^{\text {ét }} \simeq \Re_{Y}^{\text {ét }} \circ f^{*}, \quad \gamma_{f}: \mathfrak{R}_{X}^{\mathrm{e}} \circ f_{*} \simeq f_{*} \circ \mathfrak{R}_{Y}^{\mathrm{e} t},
$$




$$
\rho_{f}: f_{!} \circ \mathfrak{R}_{Y}^{\text {et }} \simeq \Re_{X}^{\text {ét }} \circ f_{!} \quad \text { et } \quad \xi_{f}: \Re_{Y}^{\text {ét }} \circ f^{!} \simeq f^{!} \circ \mathfrak{R}_{X}^{\text {ét }} .
$$

De plus, ils sont compatibles à la composition des $S$-morphismes de schémas quasi-projectifs.

(B) Pour tout $S$-schéma quasi-projectif $X$, on a un morphisme binaturel en $A, B \in \mathbf{D A}^{\mathrm{e} t}(X, \Lambda)$ :

$$
\Re_{X}^{\mathrm{et}}(\underline{\operatorname{Hom}}(A, B)) \longrightarrow \underline{\operatorname{Hom}}\left(\Re_{X}^{\mathrm{e} t}(A), \Re_{X}^{\mathrm{et}}(B)\right) .
$$

De plus, ce morphisme est inversible si A est un objet compact.

Demonstration Ceci est une conséquence immédiate du Corollaire 4.15, de la Proposition 6.2, et des Théorèmes 6.3 et 6.4 .

Proposition 6.7 - Soient $S$ un schéma de base, $\Lambda$ un anneau de coefficients et $J \subset \Lambda$ un idéal. On suppose que l'Hypothèse 6.5 est satisfaite.

(a) Soit $f: Y \longrightarrow X$ un morphisme de $S$-schémas quasi-projectifs. Alors, les foncteurs $f^{*}, f_{*}, f_{\text {! }}$ et $f^{!}$préservent les sous-catégories $\hat{\mathbf{D}}^{\mathrm{e}}\left(-, \Lambda_{J}\right) \subset \mathbf{D}^{\text {ét }}\left(-, \Lambda / J^{*}\right)$.

(b) Soient $X$ un $S$-schéma quasi-projectif et $A$ un objet de $\mathbf{D}^{\mathrm{t}}\left(X, \Lambda / J^{*}\right)$ qui est l'image d'un objet constructible de $\mathbf{D A}^{\mathrm{e} t}(S, \Lambda)$ par le foncteur de réalisation étale. Alors, l'endofoncteur $\underline{\operatorname{Hom}}(A,-)$ de $\mathbf{D}^{\mathrm{ét}}\left(X, \Lambda / J^{*}\right)$ préserve la sous-catégorie $\hat{\mathbf{D}}^{\mathrm{e}}\left(X, \Lambda_{J}\right)$.

Demonstration En utilisant les équivalences de catégories $\mathbf{D}^{\text {ét }}\left(-, \Lambda / J^{*}\right) \simeq \mathbf{D A}^{\text {ét }}\left(-, \Lambda / J^{*}\right)$, on se ramène à montrer l'énoncé correspondant pour les catégories $\hat{\mathbf{D A}^{\text {et }}}\left(-, \Lambda_{J}\right)$ (cf. la preuve de la Proposition 5.8). Alors, (a) résulte de la commutation des foncteurs $-\otimes_{\Lambda / J^{s+1}}\left(\Lambda / J^{s}\right)$ avec les quatre opérations $f^{*}, f_{*}, f_{!}$et $f^{!}$(cf. le Théorème 6.3). De même, (b) résulte de la commutation des foncteurs $-\otimes_{\Lambda / J^{s+1}}\left(\Lambda / J^{s}\right)$ avec l'opération $\underline{\operatorname{Hom}}(-,-)$ (cf. le Théorème 6.4).

Remarque 6.8 - D'après ce qui précède, les sous-catégories $\hat{\mathbf{D}}^{\text {ét }}\left(-, \Lambda_{J}\right)$ définissent un sous2-foncteur homotopique stable de $\mathbf{D}^{\text {ét }}\left(-, \Lambda / J^{*}\right)$ lorsque certaines conditions techniques sont satisfaites. Le 2-foncteur homotopique stable $\hat{\mathbf{D}}^{\text {ét }}\left(-, \Lambda_{J}\right)$ ainsi obtenu est monoïdal, symétrique et unitaire. Il est aussi fermé. Toutefois, on fera attention que les opérations $\underline{\operatorname{Hom}}(A,-)$ calculées dans $\hat{\mathbf{D}}^{\text {ét }}\left(-, \Lambda_{J}\right)$ peuvent différer de ceux calculées dans $\mathbf{D}^{\text {ét }}\left(-, \Lambda / J^{*}\right)$. Cependant, elles coïncident lorsque $A$ est la réalisation étale d'un objet constructible de $\mathbf{D A}^{\text {ét }}(-, \Lambda)$ par la Proposition 6.7, (b).

Theoreme 6.9 - Soient $S$ un schéma de base, $\Lambda$ un anneau de coefficients et $J \subset \Lambda$ un idéal. On suppose que l'Hypothèse 6.5 est satisfaite. Alors, les foncteurs de réalisation étale $\mathfrak{R}^{\text {ét }}: \mathbf{D A}^{\mathrm{e} \text { et }}(-, \Lambda) \longrightarrow \hat{\mathbf{D}}^{\mathrm{et}}\left(-, \Lambda_{J}\right)($ cf. la Proposition 5.8) commutent aux six opérations de Grothendieck pour les $S$-schémas quasi-projectifs. Plus précisément, les propriétés $(\boldsymbol{A})$ et $(\boldsymbol{B})$ du Théorème 6.6 sont satisfaites pour ces foncteurs.

\section{Pureté absolue}

Dans cette section on montre comment déduire la pureté absolue dans les catégories $\mathbf{D A}{ }^{\text {ét }}(-, \Lambda)$ en combinant la pureté absolue en cohomologie étale, démontrée par Gabber, et la pureté absolue en $K$-théorie algébrique, démontrée par Quillen. Pour cela, il est utile de reformuler la pureté absolue comme une propriété de changement de base dans des carrés transversaux d'immersions fermées (cf. la Définition 7.1 et le Théorème 7.4). Le résultat de Gabber, joint au Théorème 4.1, permettra de traiter le cas des coefficients de torsion (cf. la Proposition 7.8). La méthode de Cisinski-Déglise [11] qui repose sur des travaux de Morel-Voevodsky [37] et d'autres, permet de ramener le cas des coefficients rationnels à la pureté absolue de Quillen en $K$-théorie algébrique.

Definition 7.1 - Soient $S$ un schéma de base et $\mathrm{H}: \mathrm{Sch} / S \longrightarrow \mathfrak{I R}$ un 2-foncteur homotopique stable. On se donne un carré cartésien de $S$-schémas quasi-projectifs

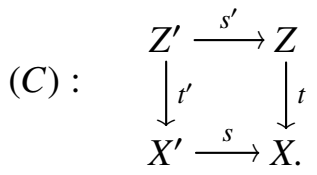


Un objet $A \in \mathrm{H}(X)$ est dit pure relativement au carré $(C)$ si le morphisme d'échange

$$
E x^{! *}: s^{* *} t^{!}(A) \longrightarrow t^{\prime} s^{*}(A)
$$

est un isomorphisme.

Le cas le plus intéressant est celui où $(C)$ est un carré transversal d'immersions fermées. Rappelons de quoi il s'agit.

Definition 7.2 - Un carré cartésien $(C)$ comme dans la Définition 7.1 est appelé un carré transversal d'immersions fermées lorsque les conditions suivantes sont satisfaites :

(a) $X, X^{\prime}, Z$ et $Z^{\prime}$ sont des schémas réguliers;

(b) $s$ et $t$ sont des immersions fermées;

(c) la codimension dans $X^{\prime}$ d'une composante irréductible de $Z^{\prime}$ est égale à la codimension dans $X$ de la composante irréductible de $Z$ qui la contient.

Dans un carré transversal d'immersions fermées $(C)$, on a $\operatorname{tor}_{\mathcal{O}_{X}}^{i}\left(s_{*} \mathcal{O}_{X^{\prime}}, t_{*} \mathcal{O}_{Z}\right)=0$ pour $i \neq 0$. Précisons aussi qu'un tel carré est supposé cartésien et pas seulement cartésien à nil-immersions près. Autrement dit, on veut que $X^{\prime} \times_{X} Z$ soit réduit.

Avant d'énoncer le résultat principal de cette section, nous introduisons les conditions (sur le schéma de base $S$ et l'anneau de coefficients $\Lambda$ ) sous lesquelles le résultat principal de ce paragraphe est valable.

НуротнÈSE 7.3 - Tout nombre premier $p$ est inversible dans $\Lambda$ ou dans $\mathcal{O}_{S}$. La dimension de Krull de $S$ est finie et les $p$-dimensions cohomologiques ponctuelles de $S$ sont uniformément bornées lorsque $p$ parcourt l'ensemble des nombres premiers non inversibles dans $\Lambda$.

D'après la Proposition 3.19, l'Hypothèse 7.3 entraine que le 2-foncteur homotopique stable $\mathbf{D A}^{\text {ét }}(-, \Lambda): \operatorname{Sch} / S \longrightarrow \mathfrak{T} \Re$ est compactement engendré par la base au sens de [4, Déf. 2.1.155].

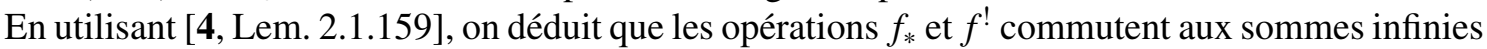
pour $f$ un $S$-morphisme quasi-projectif. Il en est de même de $f^{*}$ et $f_{!}$; pour ces opérations cette commutation est toujours vraie puisque ces dernières admettent des adjoints à droite. On peut maintenant énoncer le résultat principal de cette section.

Theoreme 7.4 - Soient $S$ un schéma régulier et $\Lambda$ un anneau de coefficients. On suppose que l'Hypothèse 7.3 est satisfaite. On se donne un carré transversal d'immersions fermées

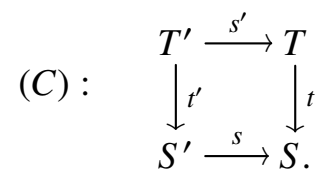

Alors, l'objet unité $\Lambda_{S}(0) \in \mathbf{D A}^{\text {ét }}(S, \Lambda)$ est pur relativement au carré $(C)$. Autrement dit, le morphisme d'échange Ex $x^{! *}: s^{\prime *} t^{!} \Lambda_{S}(0) \longrightarrow t^{\prime} s^{*} \Lambda_{S}(0)$ est inversible.

Notons tout de suite le corollaire suivant qui explique le lien entre le Théorème 7.4 et la notion usuelle de pureté, du moins localement.

Corollaire 7.5 - Soit $i: Z \hookrightarrow S$ une immersion fermée de schémas réguliers. On suppose que $Z$ est partout de codimension d et que son idéal de définition est engendré par d sections globales $g_{1}, \cdots, g_{d} \in \Gamma\left(S, \mathcal{O}_{S}\right)$. On se donne un anneau de coefficients $\Lambda$ et on suppose que l'Hypothèse 7.3 est satisfaite. On a alors un isomorphisme $i^{!} \Lambda_{S}(0) \simeq \Lambda_{Z}(-d)[-2 d]$ dans $\mathbf{D A}^{\mathrm{et}}(Z, \Lambda)$.

Demonstration On applique le Théorème 7.4 au carré transversal d'immersions fermées :

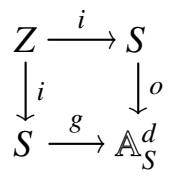

où $o$ est la section nulle et $g$ est le graphe du morphisme $\left(g_{1}, \cdots, g_{d}\right): S \longrightarrow \mathbb{A}^{d}$. 
Remarque 7.6 - Lorsque $\Lambda$ est une $\mathbb{Q}$-algèbre, le Corollaire 7.5 est dû à Cisinski-Déglise [11]. Lorsque $\Lambda=\mathbb{Z} / p$ avec $p$ un nombre premier, le Corollaire 7.5 est dû à Gabber (cf. [25, Expo. XVI]) modulo les équivalences $\mathbf{D}^{\text {ét }}(-, \mathbb{Z} / p) \simeq \mathbf{D A}^{\text {ét }}(S, \mathbb{Z} / p)$. Il est envisageable que ces deux cas particuliers suffisent pour déduire le cas général par dévissage. Cependant, pour réaliser ce dévissage il est nécessaire d'établir une compatibilité entre l'isomorphisme de pureté de Gabber et celui de Cisinski-Déglise. Nous avons choisi de faire autrement en reformulant la pureté absolue comme une propriété de changement de base. Avec cette reformulation, il est plus aisé de se ramener aux cas $\Lambda=\mathbb{Q}$ et $\Lambda=\mathbb{Z} / p$ comme le montre le résultat suivant.

Lemme 7.7 - Le Théorème 7.4 découle de ses deux cas particuliers :

(i) $\Lambda=\mathbb{Z} / p \mathbb{Z}$ avec $p$ un nombre premier inversible sur $S$;

(ii) $\Lambda=\mathbb{Q}$.

Demonstration En effet, vue la suite exacte de $\Lambda$-modules,

$$
0 \longrightarrow \Lambda_{\text {tor }} \longrightarrow \Lambda \longrightarrow \Lambda \otimes \mathbb{Q} \longrightarrow \Lambda \otimes \mathbb{Q} / \mathbb{Z} \longrightarrow 0
$$

il suffit de montrer que l'objet $\operatorname{Sus}_{T, \Sigma}^{0}\left(M_{c s t}\right) \in \mathbf{D A}^{\text {ét }}(S, \Lambda)$ est pur relativement à $(C)$ pour $M$ un $\Lambda$-module vérifiant l'une des conditions suivantes :

(i) $M$ est de torsion (en tant que $\mathbb{Z}$-module).

(ii) $M$ est uniquement divisible, i.e., c'est un module sur $\Lambda \otimes \mathbb{Q}$.

Dans le cas (i), on peut écrire $M$ comme une somme directe $M=\bigoplus_{p} M_{p}$ où $p$ parcourt l'ensemble des nombres premiers non inversibles dans $\Lambda$ et où $M_{p} \subset M$ est le sous-module des éléments annulés par une puissance de $p$. Vu que les foncteurs $s^{*}, s^{\prime *}, t^{!}$et $t^{\prime}$ commutent aux sommes infinies, on peut traiter le cas des chaque $M_{p}$ séparément. Autrement dit, on peut supposer que $M=\bigcup_{n \in \mathbb{N}} \operatorname{ker}\left(p^{n} \cdot \mathrm{id}_{M}\right)$. Pour la même raison évoquée ci-dessus, on peut même supposer que $M$ est annulé par $p^{n}$ pour un certain $n \in \mathbb{N}$. En utilisant la filtration (exhaustive et finie) de $M$ donnée par les noyaux des multiplications par $p^{r}$ (avec $r \in \mathbb{N}$ ), on se ramène en fin de compte à traiter le cas

(i') $M$ est un $\Lambda / p \Lambda$-module.

au lieu du cas (i) ci-dessus.

Notons $\Lambda^{\prime}$ la $\Lambda$-algèbre $\Lambda / p \Lambda$ ou $\Lambda \otimes \mathbb{Q}$ selon qu'on s'intéresse au cas (i') ou (ii). D'après le Corollaire A.5, le foncteur d'oubli $\mathbf{D A}^{\mathrm{e} t}\left(-, \Lambda^{\prime}\right) \longrightarrow \mathbf{D A}^{\text {ét }}(-, \Lambda)$ commute aux opérations $s^{*}$, $s^{\prime *}$. Il commute aussi aux opérations $t^{!}$et $t^{\prime}$. En effet, par adjonction on est ramené à montrer que le foncteur $-\otimes_{\Lambda} \Lambda^{\prime}: \mathbf{D A}^{\text {ét }}(-, \Lambda) \longrightarrow \mathbf{D A}^{\text {ét }}\left(-, \Lambda^{\prime}\right)$ commute aux opérations $t_{*}$ et $t_{*}^{\prime}$. Ceci découle aussitôt de l'axiome de localité et du Lemme A.3. Ainsi, si $A \in \mathbf{D A}^{\text {ét }}\left(S, \Lambda^{\prime}\right)$ est pur relativement à $(C)$, il en est de même de son image dans $\mathbf{D} \mathbf{A}^{\text {ét }}(S, \Lambda)$. Il est donc suffisant de prouver que $\operatorname{Sus}_{T, \Sigma}^{0}\left(M_{c s t}\right) \in \mathbf{D A}^{\mathrm{e}}\left(S, \Lambda^{\prime}\right)$ est pur relativement à $(C)$.

Enfin, notons $\Lambda_{0}$ l' anneau $\mathbb{Z} / p \mathbb{Z}$ ou $\mathbb{Q}$ selon qu'on s'intéresse au cas (i') ou (ii). Comme avant, le foncteur d'oubli $\mathbf{D} A^{\text {ét }}\left(-, \Lambda^{\prime}\right) \longrightarrow \mathbf{D A}^{\text {ét }}\left(-, \Lambda_{0}\right)$ commute aux opérations $s^{*}, s^{\prime *}, t^{!}$et $t^{\prime}$. De plus, d'après le Lemme A.6, ce foncteur est aussi conservatif. Il suffira donc de prouver que l'objet $\operatorname{Sus}_{T, \Sigma}^{0}\left(M_{c s t}\right) \in \mathbf{D A}^{\text {ét }}\left(S, \Lambda_{0}\right)$ est pur relativement à $(C)$. Puisque $\Lambda_{0}$ est corps, $M$ est une somme directe de copies de $\Lambda_{0}$. Étant donné que les foncteurs $s^{*}, s^{\prime *}, t^{!}$et $t^{\prime}$ commutent aux sommes, il suffit de traiter le cas $M=\Lambda_{0}$. Ceci termine la preuve du lemme.

Le cas $\Lambda=\mathbb{Z} / p \mathbb{Z}$ du Théorème 7.4 découle du résultat plus général suivant.

Proposition 7.8 - Soient $S$ un schéma régulier et $\Lambda$ une $\mathbb{Z} / N \mathbb{Z}$-algèbre avec $N \in \mathbb{N}$ un entier inversible dans $\mathcal{O}_{S}$. On suppose qu'en tout point géométrique $\bar{x}$ de $S$ la p-dimension cohomologique ponctuelle de l'hensélisé strict de $S$ en $\bar{x}$ est finie si p est un nombre premier divisant $N$. Soit $(C)$ un carré transversal d'immersions fermées comme dans le Théorème 7.4. Alors, l'objet $\Lambda_{S}(0) \in \mathbf{D A}^{\mathrm{e}}{ }^{\mathrm{t}}(S, \Lambda)$ est pur relativement à $(C)$.

Demonstration D'après le Théorème 4.1 , on a des équivalences $\mathbf{D}^{\text {ét }}(-, \Lambda) \simeq \mathbf{D A}^{\text {ét }}(-, \Lambda)$ qui commutent aux opérations de Grothendieck (cf. le Corollaire 4.15). Il suffit donc de montrer que 
$\Lambda \in \mathbf{D}^{\text {ét }}(S, \Lambda)$ est pur relativement au carré $(C)$. Il s'agit alors d'une conséquence facile du théorème de pureté absolue de Gabber (cf. [25, Expo. XVI, Th. 3.1.1]). Expliquons comment l'obtenir.

D'abord, l'énoncé à prouver est local pour la topologie étale sur $S$. On ne restreint donc pas la généralité en supposant que $S$ est affine et que $\mathcal{O}_{S}$ contient les $N$-ièmes racines de l'unité.

Supposons que le carré $(C)$ est la composition verticale de deux carrés transversaux d'immersions fermées $\left(C_{1}\right)$ et $\left(C_{2}\right)$; cette décomposition étant alors uniquement associée à une factorisation $t=t_{2} \circ t_{1}$ avec $t_{1}$ et $t_{2}$ des immersions fermées de schémas réguliers. La face carrée $E x^{* !}(C)$ s'écrit alors comme la composition horizontale des deux faces carrées $E x^{* !}\left(C_{1}\right)$ et $E x^{* !}\left(C_{2}\right)$. D'après le théorème de pureté absolue de Gabber, $t_{1}^{!} \Lambda$ est canoniquement isomorphe à $\Lambda\left(-c_{1}\right)\left[-2 c_{1}\right]$ où $c_{1}$ est la codimension de l'immersion $t_{1}$. Puisque $\mathcal{O}_{S}$ est supposé contenir les $N$-ièmes racines de l'unité, on voit que $s_{1}^{!} \Lambda$ est non canoniquement isomorphe à un shift du faisceau constant $\Lambda$. Il en découle que si $\Lambda$ est pur relativement aux carrés $\left(C_{1}\right)$ et $\left(C_{2}\right)$, il l'est aussi relativement au carré $(C)$.

La discussion précédente, jointe à l'aspect local du problème, montre qu'il suffit de traiter le cas où l'immersion fermée $t$ est partout de codimension 1 et définie par l'annulation d'une fonction $f \in \Gamma\left(S, \mathcal{O}_{S}\right)$, i.e., $\mathcal{O}_{S} /(f) \simeq t_{*} \mathcal{O}_{T}$. Dans ce cas, on a une description explicite de l'isomorphisme de pureté. C'est la suivante. Notons $u: U \hookrightarrow S$ l'immersion complémentaire à $t$ et $[f]$ la classe de $f$ dans $\mathcal{O}^{\times}(U) / \mathcal{O}^{\times}(S)$. On a alors une suite exacte courte de faisceaux étales sur Et $/ S$ :

$$
0 \longrightarrow \mathbb{G m}_{S} \longrightarrow u_{*} \mathbb{G}_{U} \longrightarrow t_{*} \mathbb{Z}[f] \longrightarrow 0 .
$$

Elle détermine une flèche $[f]: t_{*} \mathbb{Z} \longrightarrow \mathbb{G m}_{S}[1]$ dans $\mathbf{D}^{\text {t́t }}(S, \mathbb{Z})$. L'isomorphisme de pureté $\Lambda \simeq$ $t^{!} \Lambda(1)[2]$ correspond, via l'adjonction $\left(t_{*}, t^{!}\right)$, à la composition de

$$
\left.c_{f}: t_{*} \Lambda \simeq\left(t_{*} \mathbb{Z}\right)^{\mathrm{L}} \otimes \Lambda \stackrel{[f] \otimes \mathrm{id}}{\longrightarrow} G_{\mathrm{m}}[1]\right]^{\mathrm{L}} \otimes \Lambda \simeq \Lambda(1)[2] .
$$

À partir de la construction, il est clair que le diagramme

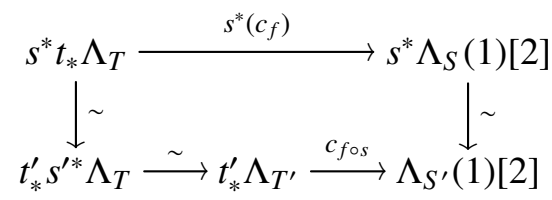

est commutatif. On déduit aussitôt un diagramme commutatif

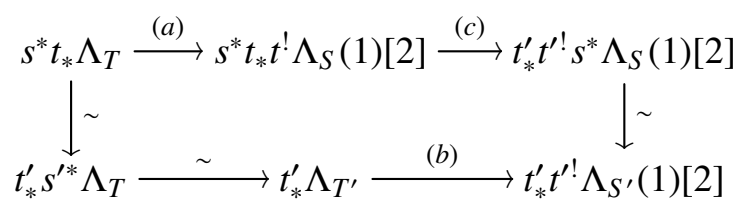

où les flèches $(a)$ et $(b)$ sont déduites de $c_{f}$ et $c_{f \circ s}$ par adjonction. D'après le théorème de pureté absolue de Gabber, ces flèches sont inversibles. (On utilise ici que $f \circ s \in \Gamma\left(S^{\prime}, \mathcal{O}_{S^{\prime}}\right)$ est un générateur de l'idéal de définition de l'immersion $t^{\prime}$.) Il s'ensuit que la flèche $(c)$ est inversible. Cette dernière est la composition du 2-isomorphisme $E x_{*}^{*}(C)$ appliqué à $t^{\prime} \Lambda_{S}$, et du 2-morphisme $E x^{\prime *}(C)$ appliqué à $\Lambda_{S}$ et auquel on applique $t_{*}^{\prime}$. Ceci termine la preuve de la proposition puisque le foncteur $t_{*}^{\prime}$ est pleinement fidèle.

Vus le Lemme 7.7 et la Proposition 7.8, il reste à prouver le Théorème 7.4 lorsque $\Lambda=\mathbb{Q}$. Comme dans [11] nous utiliserons la pureté en $K$-théorie algébrique. Les propriétés fondamentales de la $K$-théorie algébrique dont nous aurons besoins se trouvent déjà dans [38] et [17]. Le lecteur pourra aussi consulter [53, Chap. IV] pour une présentation plus lisible. On commence par quelques préliminaires.

On désigne par $\underline{\operatorname{Coh}}(X)$ une petite catégorie équivalente à la catégorie des $\mathcal{O}_{X}$-modules cohérents sur un schéma noethérien $X$. Les objets de $\underline{\operatorname{Coh}}(X)$ seront appelés des $\mathcal{O}_{X}$-modules cohérents. On supposera que $\underline{\operatorname{Coh}}(X)$ est strictement contravariante en $X$. Autrement dit, si $f: Y \longrightarrow X$ est un morphisme de schémas, on dispose d'un foncteur «image inverse » $f^{*}: \underline{\operatorname{Coh}}(X) \longrightarrow \underline{\operatorname{Coh}}(Y)$ et 
si $g: Z \longrightarrow Y$ est un autre morphisme de schémas, on a $(f \circ g)^{*}=g^{*} \circ f^{*}{ }^{\left({ }^{(8)}\right.}$ Étant donnée une immersion fermée $t: Z \hookrightarrow X$, on note $\underline{\operatorname{Coh}^{t}}(X)$ ou $\underline{\operatorname{Coh}^{Z}}(X)$ la sous-catégorie pleine de $\underline{\operatorname{Coh}}(X)$ formée des $\mathcal{O}_{X}$-modules $\mathcal{M}$ tels que tor $\overline{\mathcal{O}_{X}}\left(\mathcal{M}, t_{*} \mathcal{O}_{Z}\right)=0$ pour $i \neq 0$. C'est une sous-catégorie exacte de $\underline{\operatorname{Coh}}(X)$ stable par extensions. On note aussi $\underline{\operatorname{LL}}(X) \subset \underline{\operatorname{Coh}}(X)$ la sous-catégorie pleine des $\mathcal{O}_{X}$-modules localement libres. On a clairement $\underline{\operatorname{LL}(X)} \subset \underline{\mathrm{Coh}^{\bar{Z}}(X)}$.

Soient $S$ un schéma de base et $Z \subset S$ un sous-schéma fermé. On définit un préfaisceau de catégories exactes $\underline{\mathrm{Coh}} / S$ (resp. $\underline{\mathrm{Coh}}_{/ S}^{Z}$ ) sur $\mathrm{Sm} / S$ en associant à $X \in \mathrm{Sm} / S$ la plus petite souscatégorie exacte $\underline{\mathrm{Coh}}_{S}(X) \subset \underline{\operatorname{Coh}}(X)\left(\operatorname{resp} . \underline{\mathrm{Coh}}_{/ S}^{Z}(X) \subset \underline{\mathrm{Coh}}^{X \times_{S} Z}(X)\right)$ stable par extensions et contenant les $\mathcal{O}_{X}$-modules cohérents qui sont localement pour le topologie de Zariski isomorphes à des images inverses d'objets de $\underline{\operatorname{Coh}}(S)\left(\operatorname{resp} \cdot \underline{\operatorname{Coh}^{Z}}(S)\right)$ suivant le morphisme structural $X \longrightarrow S$. Si $f: Y \longrightarrow X$ est un morphisme de $S$-schémas lisses, le foncteur $f^{*}: \underline{\operatorname{Coh}}_{/ S}(X) \longrightarrow \underline{\operatorname{Coh}}_{/ S}(Y)$ est exact. On note aussi $\underline{\mathrm{LL}}_{/ S}(-) \subset \underline{\operatorname{Coh}}_{/ S}^{Z}(-)$ le sous-préfaisceau donné en $X \in \mathrm{Sm} / S$ par $\underline{\mathrm{LL}}(X)$.

Étant donnée une petite catégorie exacte $\mathcal{E}$, on note $\mathrm{Q}(\mathcal{E})$ la catégorie ayant les mêmes objets que $\mathcal{E}$ et où les flèches de $A \in \mathcal{E}$ vers $A^{\prime} \in \mathcal{E}$ sont données par des classes d'isomorphismes de triplets $\left(B, s: B \rightarrow A, i: B \hookrightarrow A^{\prime}\right)$ avec $B \in \mathcal{E}, s$ un epimorphisme admissible et $i$ un monomorphisme admissible. Deux tels triplets $\left(B, B \rightarrow A, B \hookrightarrow A^{\prime}\right)$ et $\left(C, C \rightarrow A, C \hookrightarrow A^{\prime}\right)$ sont isomorphes s'il existe un isomorphisme $B \simeq C$ faisant commuter le diagramme qu'on pense. Pour plus de détails, le lecteur est renvoyé à [38, §2, p. 100] (voir aussi [53, Chap. IV, §6]). L'espace de $K$-théorie de Quillen associé à $\mathcal{E}$ est l'ensemble simplicial $\mathrm{R} \Omega_{s}(\mathrm{~N}(\mathrm{Q} \mathcal{E}))$ qu'on notera $\mathrm{K}(\mathcal{E})$. Ici, $\mathrm{N}(-)$ est le nerf d'une catégorie et $\mathrm{R} \Omega_{s}=\Omega_{s} \circ(-)_{f i b}$ avec $\Omega_{s}=\underline{\operatorname{hom}}\left(\Delta^{1} / \partial \Delta^{1},-\right)$ et $(-)_{f i b}$ un foncteur de remplacement fibrant dans la catégorie des ensembles simpliciaux pointés $\Delta^{\text {op }} \mathcal{E} n s_{\bullet}$ munie de sa structure de modèles usuelle. (On peut prendre pour $(-)_{f i b}$ le composé du foncteur « réalisation topologique » avec le foncteur «ensemble simplicial singulier associé ».) La $K$-théorie de Quillen $\mathrm{K}(-)$, ainsi définie, est covariante par rapport aux foncteurs exacts. On en déduit ainsi des préfaisceaux d'ensembles simpliciaux $\mathrm{K}\left(\underline{\mathrm{LL}}_{/ S}(-)\right), \mathrm{K}\left(\underline{\mathrm{Coh}}_{/ S}^{Z}(-)\right)$ et $\mathrm{K}\left(\underline{\mathrm{Coh}}_{/ S}(-)\right)$ sur $\mathrm{Sm} / S$.

Lemme 7.9 - Supposons que S est régulier et de dimension de Krull finie. Alors, les inclusions $\underline{\mathrm{LL}}_{/ S}(-) \hookrightarrow \underline{\mathrm{Coh}}_{/ S}^{Z}(-) \hookrightarrow \underline{\mathrm{Coh}}_{/ S}(-)$ induisent des équivalences d'homotopie simpliciales de préfaisceaux

$$
\mathrm{K}\left(\underline{\mathrm{LL}}_{/ S}(-)\right) \longrightarrow \mathrm{K}\left(\underline{\mathrm{Coh}}_{/ S}^{Z}(-)\right) \longrightarrow \mathrm{K}\left(\underline{\mathrm{Coh}}_{/ S}(-)\right) .
$$

Demonstration Ceci est une conséquence immédiate de [38, §4, Cor. 1] et du fait que tout $\mathcal{O}_{X^{-}}$ module cohérent sur $X \in \mathrm{Sm} / S$ admet une résolution de longueur finie par des $\mathcal{O}_{X}$-modules localement libres.

Propositron 7.10 - Supposons que S est régulier et de dimension de Krull finie. Les préfaisceaux d'ensembles simpliciaux $\mathrm{K}\left(\underline{\mathrm{LL}}_{/ S}(-)\right), \mathrm{K}\left(\underline{\mathrm{Coh}}_{/ S}^{Z}(-)\right)$ et $\mathrm{K}\left(\underline{\mathrm{Coh}}_{/ S}(-)\right)$ sont $\left(\mathbb{A}^{1}\right.$, Nis)-fibrants.

Demonstration Ceci est essentiellement bien connu. D'après le Lemme 7.9, il suffit de considérer le cas de $\mathrm{K}\left(\underline{\mathrm{LL}}_{/ S}(-)\right)$. Vu [38, 6 , Cor. of Th. 8], il suffit de montrer que $\mathrm{K}\left(\underline{\mathrm{LL}}_{/ S}(-)\right)$ possède la propriété de Brown-Gersten dans les carrés distingués Nisnevich. Cette propriété se vérifie sur le petit site étale $\mathrm{Et} / X$ de chaque $X \in \mathrm{Sm} / S$. Or, d'après [38, $\S 4$, Cor. 1], on dispose d'une équivalence d'homotopie simpliciale de préfaisceaux $\mathrm{K}\left(\underline{\mathrm{LL}}_{/ S}(-)\right)_{\mid \mathrm{Et} / X} \longrightarrow \mathrm{K}(\underline{\mathrm{Coh}}(-))_{\mid \mathrm{Et} / X}$. On utilise alors [38, §7, Prop. 3.2] pour conclure.

Notons $\mathbb{G} r_{S}(d \mid r)$ la grassmannienne relative sur $S$ qui classifie les quotients localement libres de rang $r$ du module libre de rang $d+r$. On dispose d'une suite exacte universelle de modules

8. On peut prendre pour $\underline{\operatorname{Coh}}(X)$ la catégorie suivante. Un objet est un uplet $\left(\left(U_{i}\right)_{1 \leq i \leq n},\left(d_{i}\right)_{1 \leq i \leq n},\left(\mathcal{K}_{i}\right)_{1 \leq i \leq n},\left(u_{i j}\right)_{1 \leq i, j \leq n}\right)$ tel que $n \in \mathbb{N},\left(U_{i}\right)_{1 \leq i \leq n}$ est un recouvrement ouvert de $X,\left(d_{i}\right)_{1 \leq i \leq n} \in \mathbb{N}^{n}, \mathcal{K}_{i} \subset \mathcal{O}_{U_{i}}^{d_{i}}$ un sous- $\mathcal{O}_{U_{i}}$-module cohérent pour tout $1 \leq i \leq n$ et $u_{i j}:\left(\mathcal{O}_{U_{i}}^{d_{i}} / \mathcal{K}_{i}\right)_{\mid U_{i} \cap U_{j}} \simeq\left(\mathcal{O}_{U_{j}}^{d_{j}} / \mathcal{K}_{j}\right)_{\mid U_{i} \cap U_{j}}$ un isomorphisme de $\mathcal{O}_{U_{i} \cap U_{j}}$-modules pour tout $1 \leq i, j \leq n$. Bien entendu, les $u_{i j}$ sont supposés satisfaire à la relation de cocycle habituelle. À un tel uplet, on peut associer par recollement un $\mathcal{O}_{X}$-module cohérent. Les morphismes dans $\underline{\operatorname{Coh}}(X)$ sont alors les morphismes entres les $\mathcal{O}_{X}$-modules cohérents associés à ces uplets. 
localement libres sur $\mathbb{G} r_{S}(d \mid r)$ :

$$
0 \longrightarrow \mathcal{U}_{d, r} \longrightarrow \mathcal{O}^{d+r} \longrightarrow \mathcal{V}_{d, r} \longrightarrow 0
$$

On a des inclusions évidentes $\mathbb{G} r_{S}(d \mid r) \hookrightarrow \mathbb{G} r_{S}(1+d \mid r)$ et $\mathbb{G} r_{S}(d \mid r) \hookrightarrow \mathbb{G} r_{S}(d \mid r+1)$ qui correspondent respectivement à $\mathcal{V}_{d, r}$ comme un quotient de $\mathcal{O}^{1+d+r}$ et à $\mathcal{V}_{d, r} \oplus \mathcal{O}$ comme un quotient de $\mathcal{O}^{d+r+1}$. Ces inclusions déterminent un système inductif filtrant paramétré par $(d, r) \in \mathbb{N} \times \mathbb{N}$. La colimite de ce système, prise dans la catégorie des préfaisceaux d'ensembles sur $\mathrm{Sm} / S$, sera notée $\mathbb{G} r_{S}(\infty \mid \infty)$. L'énoncé suivant est essentiellement dû à Morel et Voevodsky (cf. [37, Th. 3.13]). Il apporte toutefois une précision supplémentaire qui sera utile dans la suite. Cette précision figure également dans [40, Prop. 3.1.5].

Theoreme 7.11 - Soit $S$ un schéma régulier de dimension de Krull finie. Il existe un isomorphisme canonique

$$
\mathbb{Z} \times \mathbb{G} r_{S}(\infty \mid \infty) \stackrel{\sim}{\longrightarrow} \mathrm{K}\left(\underline{\mathrm{LL}}_{/ S}(-)\right)
$$

dans $\mathbf{H}_{\bullet}(S)=\mathbf{H o}_{\mathbb{A}^{1}-\mathrm{Nis}}\left(\mathbf{P S h}\left(\operatorname{Sm} / S, \Delta^{\mathrm{op}} \mathcal{E} n s_{\bullet}\right)\right)$. Pour $n \in \mathbb{Z}$ et $(d, r) \in \mathbb{N} \times \mathbb{N}$, la composition de

$$
\mathbb{G} r_{S}(d \mid r) \simeq\{n\} \times \mathbb{G} r_{S}(d \mid r) \hookrightarrow \mathbb{Z} \times \mathbb{G} r_{S}(\infty \mid \infty) \stackrel{\sim}{\longrightarrow} \mathrm{K}\left(\underline{\mathrm{LL}}_{/ S}(-)\right)
$$

correspond à $n-d+\left[\mathcal{U}_{d, r}\right]$ dans $\mathrm{K}_{0}\left(\mathbb{G} r_{S}(d \mid r)\right)$.

Demonstration Soit $X$ un $S$-schéma lisse. Notons $\underline{L_{L}}(X)$ la catégorie exacte ayant $\underline{\operatorname{LL}}(X)$ comme catégorie sous-jacente mais où les suites exactes sont données par les suites exactes courtes scindées. On obtient ainsi un préfaisceau de catégories exactes $\underline{\mathrm{LL}}_{/ S}^{\prime}(-)$ sur $\mathrm{Sm} / S$. Le morphisme identité $\underline{\mathrm{LL}}_{/ S}^{\prime}(-) \longrightarrow \underline{\mathrm{LL}}_{/ S}(-)$ fournit un morphisme de préfaisceaux en ensembles simpliciaux pointés :

$$
\mathrm{K}\left(\underline{\mathrm{LL}}_{/ S}^{\prime}(-)\right) \longrightarrow \mathrm{K}\left(\underline{\mathrm{LL}}_{/ S}(-)\right) .
$$

Lorsqu'on évalue (61) en un $S$-schéma affine et lisse, on obtient un isomorphisme. (En effet, sur un schéma affine toute suite exacte de modules localement libres est scindée.) Il s'ensuit que (61) est une équivalence Nis-locale.

Par ailleurs, la $K$-théorie d'une catégorie additive admet une autre description fournie par la construction $\mathrm{S}^{-1} \mathrm{~S}$ de Quillen. Expliquons de quoi il s'agit. Soit $\mathcal{C}$ une petite catégorie additive. On note $\mathrm{S}^{-1} \mathrm{~S}(\mathcal{C})$ la catégorie ayant pour objets des couples $(A, B)$ d'objets de $\mathcal{C}$ et pour morphismes entre $(A, B)$ et $(C, D)$ les classes d'isomorphismes de triplets $(U, a: A \oplus U \simeq C, b: B \oplus U \simeq D)$. Ce triplet est isomorphe à $\left(U^{\prime}, a^{\prime}: A \oplus U^{\prime} \simeq C, b^{\prime}: B \oplus U^{\prime} \simeq D\right)$ s'il existe un isomorphisme $e: U \simeq U^{\prime}$ tel que $a=a^{\prime} \circ(\mathrm{id} \oplus e)$ et $b=b^{\prime} \circ(\mathrm{id} \oplus e)$. (Pour plus de détails, le lecteur est renvoyé à [17] ou à [53, Chap. IV, §4].) On pose alors $\mathrm{K}^{\oplus}(\mathcal{C})=\mathrm{N}\left(\mathrm{S}^{-1} \mathrm{~S}(\mathcal{C})\right.$ ). D'après [17] (voir aussi [53, Chap. IV, Th. 7.1]), il existe un isomorphisme $\mathrm{K}^{\oplus}(\mathcal{C}) \simeq \mathrm{K}(\mathcal{C})$ dans $\mathbf{H o}\left(\Delta^{\mathrm{op}}\left(\mathcal{E} n s_{\bullet}\right)\right)$. La construction de cet isomorphisme est suffisamment fonctorielle pour fournir un isomorphisme

$$
\left.\mathrm{K}^{\oplus} \underline{\mathrm{LL}}_{/ S}(X)\right) \simeq \mathrm{K}\left(\underline{\mathrm{LL}}_{/ S}^{\prime}(X)\right)
$$

dans $\mathbf{H o}_{s}\left(\mathbf{P S h}\left(\operatorname{Sm} / S, \Delta^{\mathrm{op}} \mathcal{E} n s_{\bullet}\right)\right)$.

On considère à présent les sous-catégories additives pleines $\underline{\mathrm{L}}(X) \subset \underline{\mathrm{LL}}(X)$ ayant pour objets les modules libres standards $\mathcal{O}_{X}^{n}$ pour $n \in \mathbb{N}$. On obtient ainsi un sous-préfaisceau de catégories additives $\underline{\mathrm{L}}_{/ S}(-) \subset \underline{\mathrm{LL}}_{/ S}(-)$. D'où un morphisme de préfaisceaux d'ensembles simpliciaux :

$$
\mathrm{K}^{\oplus}\left(\underline{\mathrm{L}}_{/ S}(X)\right) \longrightarrow \mathrm{K}^{\oplus}\left(\underline{\mathrm{LL}}_{/ S}(X)\right) .
$$

Étant donné que tout module localement libre sur un schéma local est libre, on déduit aussitôt que (63) est une équivalence Nis-locale.

On note $\mathbb{G} \ell_{n, S}$ le groupe linéaire relatif sur $S$. On dispose d'unclusions $\mathbb{G} \ell_{n, S} \hookrightarrow \mathbb{G} \ell_{n+1, S}$ données par $-\oplus$ id. On note $\mathbb{G} \ell_{\infty, S}$ la colimite suivant $n \in \mathbb{N}$ des $\mathbb{G} \ell_{n, S}$ prise dans la catégorie des préfaisceaux de groupes sur $\mathrm{Sm} / S$. On dispose d'un morphisme évident

$$
\bigsqcup_{n \in \mathbb{N}} \mathrm{N}\left(\mathbb{G} \ell_{n, S}\right) \longrightarrow \mathrm{K}^{\oplus}\left(\underline{\mathrm{L}}_{/ S}(X)\right) \text {. }
$$


Sur N(G $\left.\ell_{n, S}\right)$, il est induit par l'identification de $\mathbb{G} \ell_{n, S}(X) \operatorname{avec} \operatorname{end}_{\mathrm{S}^{-1} \mathrm{~S}(\mathrm{~L}(X))}\left(\mathcal{O}_{X}^{n}, 0\right)$. D'après [17] (voir aussi [53, Chap. IV, Th. 4.9]) le morphisme (64) est une groupe-complétion. On déduit alors un isomorphisme

$$
\mathrm{R} \Omega_{s} \mathrm{~N}\left(\coprod_{n \in \mathbb{N}} \mathrm{N}\left(G \ell_{n, S}\right)\right) \stackrel{\sim}{\longrightarrow} \mathrm{K}^{\oplus}\left(\underline{\mathrm{L}}_{/ S}(X)\right)
$$

dans H.(S). Or, d'après [37, Prop. 3.7 et Prop. 3.10], on a des isomorphismes canoniques

$$
\mathbb{Z} \times \mathbb{G} r_{S}(\infty \mid \infty) \stackrel{\sim}{\longrightarrow} \mathbb{Z} \times \mathrm{N} G \ell_{\infty, S} \stackrel{\sim}{\longrightarrow} \mathrm{R} \Omega_{s}^{1} \mathrm{~N}\left(\coprod_{n \in \mathbb{N}} \mathrm{N}\left(\mathbb{G} \ell_{n, S}\right)\right)
$$

dans H.(S). En composant les isomorphismes (61), (62), (63), (65) et (66) dans H.( $(S)$, on obtient l'isomorphisme (60) de l'énoncé. La seconde assertion découle des constructions. Sa vérification est laissée au lecteur.

Soit $s: S^{\prime} \hookrightarrow S$ une immersion fermée. On dispose d'un morphisme de préfaisceaux de catégories exactes :

$$
\underline{\mathrm{Coh}}_{/ S}^{S^{\prime}}(-) \longrightarrow S_{*} \underline{\mathrm{Coh}}_{/ S^{\prime}}(-)
$$

En $X \in \mathrm{Sm} / S$, il est induit par le foncteur exact

$$
-\otimes_{\mathcal{O}_{S}} s_{*} \mathcal{O}_{S^{\prime}}: \underline{\mathrm{Coh}}_{S}^{S^{\prime}}(X) \longrightarrow \underline{\mathrm{Coh}}_{/ S^{\prime}}\left(X \times_{S} S^{\prime}\right)
$$

En passant à la $K$-théorie de Quillen, on obtient un morphisme de préfaisceaux d'ensembles simpliciaux sur $\mathrm{Sm} / S$ :

$$
\mathrm{K}\left(\underline{\mathrm{Coh}}_{/ S}^{S^{\prime}}(-)\right) \longrightarrow s_{*} \mathrm{~K}\left(\underline{\mathrm{Coh}}_{/ S^{\prime}}(-)\right)
$$

Le résultat ci-dessous est une conséquence facile (et bien connue) du théorème de Morel et Voevodsky [37, Th. 3.13] comme énoncé dans le Théorème 7.11.

Corollaire 7.12 - Supposons que $S$ et $S^{\prime}$ sont des schémas réguliers et que la dimension de Krull de $S$ est finie. Alors, le morphisme canonique $\mathrm{L} s^{*} \mathrm{~K}\left(\underline{\mathrm{Coh}}_{/ S}^{S^{\prime}}(-)\right) \longrightarrow \mathrm{K}\left(\underline{\mathrm{Coh}}_{/ S}(-)\right)$, déduit de (68) via l'adjonction $\left(\mathrm{L} s^{*}, s_{*}\right)$, est inversible dans $\mathbf{H} .\left(S^{\prime}\right)$.

Demonstration D'après le Lemme 7.9 et le Théorème 7.11, on a un carré dans $\mathbf{H} .(S)$

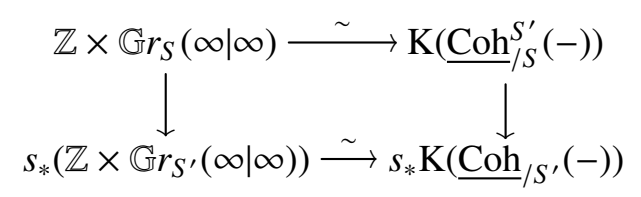

où les flèches horizontales sont inversibles. Étant donné que le préfaisceau $\mathbb{Z} \times \mathbb{G} r_{S}(\infty \mid \infty)$ est une colimite filtrante de préfaisceaux représentables, on a des isomorphismes

$$
\operatorname{Ls} s^{*}\left(\mathbb{Z} \times \mathbb{G} r_{S}(\infty \mid \infty)\right) \simeq s^{*}\left(\mathbb{Z} \times \mathbb{G} r_{S}(\infty \mid \infty)\right) \simeq \mathbb{Z} \times \mathbb{G} r_{S^{\prime}}(\infty \mid \infty) .
$$

Autrement dit, le morphisme canonique $L s^{*}\left(\mathbb{Z} \times \mathbb{G} r_{S}(\infty \mid \infty)\right) \longrightarrow \mathbb{Z} \times \mathbb{G} r_{S^{\prime}}(\infty \mid \infty)$ est inversible. Pour conclure, il suffit donc de savoir que la carré ci-dessus est commutatif. Or,

$$
\operatorname{hom}_{\mathbf{H} .(S)}\left(\mathbb{Z} \times \mathbb{G} r_{S}(\infty \mid \infty), s_{*} \mathrm{~K}\left(\underline{\mathrm{Coh}}_{/ S^{\prime}}(-)\right)\right)=\lim _{d, r \in \mathbb{N}} \operatorname{hom}_{\mathbf{H} \text {. }(S)}\left(\mathbb{Z} \times \mathbb{G} r_{S}(d \mid r), s_{*} \mathrm{~K}\left(\underline{\mathrm{Coh}}_{/ S^{\prime}}(-)\right)\right) \text {. }
$$

Ceci découle de [40, Lem. 1.2.1 et Lem. 1.2.10] en utilisant l'adjonction $\left(L s^{*}, s_{*}\right)$, l'isomorphisme (69) et le Théorème 7.11. On est ainsi ramené à montrer que les carrés

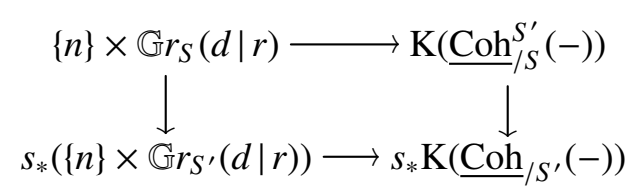

commutent pour tous les triplets $(n, d, r) \in \mathbb{Z} \times \mathbb{N} \times \mathbb{N}$. Ceci découle trivialement de la seconde partie du Théorème 7.11. 
Dans la suite, on prendra pour $\mathrm{R} \Omega_{s}^{1}(X)$ l'ensemble simplicial singulier associé à l'espace des lacets dans la réalisation topologique de $X \in \Delta^{\mathrm{op}}\left(\mathcal{E} n s_{\bullet}\right)$. On dispose alors d'une loi de composition interne

$$
+: \mathrm{R} \Omega_{s}^{1}(X) \times \mathrm{R} \Omega_{s}^{1}(X) \longrightarrow \mathrm{R} \Omega_{s}^{1}(X),
$$

fonctorielle en $X$, induite par la concaténation des lacets. Le lacet constant induit un morphisme d'ensemble simpliciaux $0: \Delta^{0} \longrightarrow \Omega_{s}^{1}(X)$ qui est une unité à homotopie près pour la loi (70). Autrement dit, on a des homotopies simpliciales $0+\mathrm{id}_{\mathrm{R} \Omega_{s}^{1}(X)} \sim \mathrm{id}_{\mathrm{R}_{s}^{1}(X)}$ et $\mathrm{id}_{\mathrm{R}_{s}^{1}(X)}+0 \sim \mathrm{id}_{\mathrm{R} \Omega_{s}^{1}(X)}$ qui sont d'ailleurs fonctorielles en $X$. La loi (70) admet aussi des inverses canoniques à homotopie près. Ainsi on a un morphisme $\operatorname{inv}_{X}: \mathrm{R} \Omega_{s}^{1}(X) \longrightarrow \Omega_{s}^{1}(X)$ et des homotopies $\operatorname{id}_{\mathrm{R} \Omega_{s}^{1}(X)}+\operatorname{inv}_{X} \sim 0$ et $\operatorname{inv}_{X}+\operatorname{id}_{\mathrm{R}_{s}^{1}(X)} \sim 0$; le tout est fonctoriel en $X$. Le morphisme composé $+\circ\left(\operatorname{id}_{\mathrm{R}_{s}^{1}(X)} \times \operatorname{inv}_{X}\right)$ sera simplement noté

$$
-: \mathrm{R} \Omega_{s}^{1}(X) \times \mathrm{R} \Omega_{s}^{1}(X) \longrightarrow \mathrm{R} \Omega_{s}^{1}(X) .
$$

Lui aussi, il est fonctoriel en $X$.

Étant donnée une catégorie exacte $\mathcal{E}$, l'espace de $K$-théorie de Quillen $\mathrm{K}(\mathcal{E})=\mathrm{R} \Omega_{s}^{1}(\mathrm{~N}(\mathrm{Q}(\mathcal{E}))$ ) acquiert les structures décrites ci-dessus. De plus, elles sont fonctorielles en les foncteurs exacts. Nous utiliserons ceci pour construire des $T$-spectres de $K$-théorie $\mathbf{K}\left(\underline{\operatorname{LL}}_{/ S}(-)\right), \mathbf{K}\left(\underline{\operatorname{Coh}}_{/ S}^{Z}(-)\right)$ et $\mathbf{K}\left(\underline{\mathrm{Coh}}_{S}(-)\right)$. La construction étant la même dans les trois cas, nous nous concentrerons sur celui du préfaisceau $\underline{\mathrm{Coh}}_{/ S}^{Z}(-)$. Nous prendrons pour $T_{S}$ le remplacement projectivement cofibrant du préfaisceau pointé $\left(\mathbb{P}_{S}^{1}, \infty_{S}\right)$ donné par $\Delta^{1} \amalg_{\Delta^{0}} \mathbb{P}_{S}^{1}$ pointé par $0 \in \Delta^{1}$; la somme amalgamée est prise suivant $1: \Delta^{0} \rightarrow \Delta^{1}$ et $\infty: \Delta^{0} \rightarrow \mathbb{P}_{S}^{1}$. Pour $X \in \mathrm{Sm} / S$, on dispose de deux foncteurs exacts $\mathrm{f}_{X}^{0}, \mathrm{f}_{X}^{1}: \underline{\operatorname{Coh}}_{/ S}^{Z}(X) \longrightarrow \underline{\operatorname{Coh}}_{/ S}^{Z}\left(\mathbb{P}_{X}^{1}\right)$. Si $\pi: \mathbb{P}_{X}^{1} \longrightarrow X$ est la projection évidente et $\mathcal{O}_{\mathbb{P}_{X}^{1}}(1)$ est le $\mathcal{O}_{\mathbb{P}_{X}^{1}}$-module tautologique sur $\mathbb{P}_{X}^{1}$, alors $f_{X}^{0}=\pi^{*}(-)$ et $\mathrm{f}_{X}^{1}=\mathcal{O}_{\mathbb{P}_{X}^{1}}(1) \otimes_{\mathcal{O}_{\mathbb{P}_{X}^{1}}} \pi^{*}(-)$. On en déduit deux morphismes de préfaisceaux d'ensembles simpilciaux

$$
\mathrm{f}^{0}, \mathrm{f}^{1}: \mathrm{K}\left(\underline{\operatorname{Coh}}_{/ S}^{Z}(-)\right) \longrightarrow \underline{\operatorname{hom}}\left(\mathbb{P}_{S}^{1}, \mathrm{~K}\left(\underline{\operatorname{Coh}}_{/ S}^{Z}(-)\right)\right) \text {. }
$$

En utilisant (71), on peut alors former le morphisme de préfaisceaux d'ensembles simpliciaux

$$
\mathrm{f}^{0}-\mathrm{f}^{1}: \mathrm{K}\left(\underline{\operatorname{Coh}}_{/ S}^{Z}(-)\right) \longrightarrow \underline{\operatorname{hom}}\left(\mathbb{P}_{S}^{1}, \mathrm{~K}\left(\underline{\operatorname{Coh}}_{/ S}^{Z}(-)\right)\right) .
$$

Le choix d'une identification $\infty^{*}\left(\mathcal{O}_{\mathbb{P}^{1}}\right)=\infty^{*}\left(\mathcal{O}_{\mathbb{P}^{1}}(1)\right)$ fournit une homotopie simpliciale entre l'application nulle et la composition de

$$
\mathrm{K}\left(\underline{\operatorname{Coh}}_{/ S}^{Z}(-)\right) \stackrel{\mathrm{f}^{0}-\mathrm{f}^{1}}{\longrightarrow} \underline{\operatorname{hom}}\left(\mathbb{P}_{S}^{1}, \mathrm{~K}\left(\underline{\operatorname{Coh}}_{/ S}^{Z}(-)\right)\right) \stackrel{\infty^{*}}{\longrightarrow} \mathrm{K}\left(\underline{\operatorname{Coh}}_{/ S}^{Z}(-)\right) .
$$

Il s'ensuit un morphisme canonique

$$
\gamma^{\prime}: \mathrm{K}\left(\underline{\operatorname{Coh}}_{/ S}^{Z}(-)\right) \longrightarrow \underline{\operatorname{hom}}\left(T_{S}, \mathrm{~K}\left(\underline{\operatorname{Coh}}_{/ S}^{Z}(-)\right)\right) .
$$

Le $T$-spectre $\mathbf{K}\left(\underline{\operatorname{Coh}}_{/ S}^{Z}(-)\right)$ est donné en tout niveau par $\mathrm{K}\left(\underline{\operatorname{Coh}}_{/ S}^{Z}(-)\right)$ et admet le morphisme $\gamma$ : $T_{S} \wedge \mathrm{K}\left(\underline{\operatorname{Coh}}_{/ S}^{Z}(-)\right) \longrightarrow \mathrm{K}\left(\underline{\operatorname{Coh}}_{/ S}^{Z}(-)\right)$, déduit par adjonction de (72), pour morphisme d'assemblage.

Propositron 7.13 - Soit $S$ un schéma régulier de dimension de Krull finie. Le T-spectre $\mathbf{K}\left(\underline{\mathrm{Coh}}_{/ S}^{Z}(-)\right)$ est projectivement stablement $\left(\mathbb{A}^{1}, \mathrm{Nis}\right)$-fibrant. Il en est de même de $\mathbf{K}\left(\underline{\mathrm{LL}}_{/ S}(-)\right)$ et $\mathbf{K}\left(\underline{\mathrm{Coh}}_{/ S}(-)\right)$.

Demonstration Vue la Proposition 7.10, il reste à montrer que (72) est une équivalence d'homotopie de préfaisceaux simpliciaux. Ceci est une conséquence immédiate du théorème du fibré projectif (cf. [38, §8, Th. 2.1]).

Soit $t: T \hookrightarrow S$ une immersion fermée. Pour tout $X \in \mathrm{Sm} / S$, le foncteur « image directe » $\left(\operatorname{id}_{X} \times_{S} t\right)_{*}: \underline{\mathrm{Coh}}_{/ T}\left(X \times_{S} T\right) \longrightarrow \underline{\mathrm{Coh}}_{/ S}(X)$ est exact. On a donc un morphisme de préfaisceaux de catégories exactes $t_{*}: \underline{\mathrm{Coh}}_{/ T}(-) \longrightarrow \underline{\mathrm{Coh}}_{/ S}(-)$ qui induit un morphisme de $T$-spectres

$$
t_{*} \mathbf{K}\left(\underline{\mathrm{Coh}}_{/ T}(-)\right) \longrightarrow \mathbf{K}\left(\underline{\mathrm{Coh}}_{/ S}(-)\right) \text {. }
$$


On a le résultat suivant qui n'est rien de plus qu'une reformulation de la pureté de Quillen en $K$-théorie algébrique des schémas.

LEMme 7.14 - Soit $t: T \hookrightarrow S$ une immersion fermée entre schémas réguliers de dimension de Krull finie. Alors, le morphisme $\mathbf{K}\left(\underline{\mathrm{Coh}}_{/ T}(-)\right) \longrightarrow t^{\prime} \mathbf{K}\left(\underline{\mathrm{Coh}}_{/ S}(-)\right)$, déduit par l'adjonction $\left(t_{*}, t^{\prime}\right)$ du morphisme (73), est un isomorphisme dans $\mathbf{S H}(T)$.

Demonstration Soit $j: U \hookrightarrow S$ l'immersion ouverte complémentaire à $t$. Vu que $\mathbf{K}\left(\underline{\mathrm{Coh}}_{/ U}(-)\right)$ est projectivement stablement ( $\mathbb{A}^{1}$, Nis)-fibrant (cf. la Proposition 7.13), on a un isomorphisme $j_{*} \mathbf{K}\left(\underline{\mathrm{Coh}}_{J}(-)\right) \simeq \mathrm{R} j_{*} \mathbf{K}\left(\underline{\mathrm{Coh}}_{U}(-)\right)=\mathrm{R} j_{*} j^{*} \mathbf{K}\left(\underline{\mathrm{Coh}}_{/ S}(-)\right)$. D'après [4, Prop. 1.4.9], il suffit donc de montrer que le morphisme canonique

$$
t_{*} \mathbf{K}\left(\underline{\mathrm{Coh}}_{/ T}(-)\right) \longrightarrow \operatorname{HoFib}\left\{\mathbf{K}\left(\underline{\mathrm{Coh}}_{/ S}(-)\right) \rightarrow j_{*} \mathbf{K}\left(\underline{\mathrm{Coh}}_{/ U}(-)\right)\right\}
$$

est inversible dans $\mathbf{S H}(S)$. Il suffit de faire cela niveau par niveau et au-dessus de $X \in \mathrm{Sm} / S$. Si $Y=X \times_{S} T$ et $V=X \times_{S} U$, il suffit alors de montrer que

$$
\mathrm{K}(\underline{\mathrm{Coh}}(Y)) \longrightarrow \operatorname{HoFib}\{\mathrm{K}(\underline{\operatorname{Coh}}(X)) \rightarrow \mathrm{K}(\underline{\operatorname{Coh}}(V))\}
$$

est une équivalence d'homotopie. Ceci découle aussitôt de [38, §5, Th. 4 et Th. 5].

Remarque 7.15 - Strictement parlant, le $T$-spectre $\mathbf{K}\left(\underline{\mathrm{Coh}}_{S}(-)\right)$ n'est pas un objet de la catégorie $\mathbf{S H}_{\mathfrak{M}}(S)$ (pour $\mathfrak{M}=\mathbf{S p t}_{\mathrm{S}^{1}}^{\Sigma}\left(\Delta^{\mathrm{op}} \mathcal{E} n_{\bullet}\right)$ ) construite et étudiée dans [5, Chap. 4] (cf. l'Annexe A). C'est plutôt un objet de la catégorie $\mathbf{S H}(S)$ construite par Jardine dans [28]. Toutefois, en pratique, il n'est pas dangereux de confondre ces deux catégories. En effet, le foncteur

$$
\operatorname{Spt}_{T}\left(\mathbf{P S h}\left(\operatorname{Sm} / S, \Delta^{\mathrm{op}} \mathcal{E} n s_{\bullet}\right)\right) \longrightarrow \operatorname{Spt}_{T}\left(\mathbf{P S h}\left(\operatorname{Sm} / S, \operatorname{Spt}_{\mathrm{S}^{1}}^{\Sigma}\left(\Delta^{\mathrm{op}} \mathcal{E} n s_{\bullet}\right)\right)\right),
$$

induit par le foncteur de suspension infinie au niveau des ensembles simpliciaux, est une équivalence de Quillen à gauche. Il induit alors une équivalence entre la catégorie $\mathbf{S H}(S)$ de $[\mathbf{2 8}]$ et celle de [5]. Dans la suite, nous passerons sous silence cette distinction.

Theoreme 7.16 - Soit $S$ un schéma régulier. Alors, $\mathbf{K}\left(\underline{\mathrm{Coh}}_{/ S}(-)\right) \in \mathbf{S H}(S)$ est pur relativement à tout carré transversal d'immersions fermées $(C)$ comme dans le Théorème 7.4.

Demonstration On a un carré commutatif de $T$-spectres :

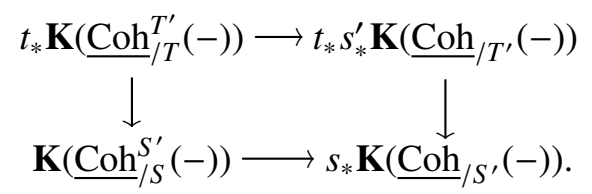

Les flèches verticales sont induites par les foncteurs images directes

$$
\left(\operatorname{id}_{X} \times t\right)_{*}: \underline{\operatorname{Coh}}_{/ T}^{T^{\prime}}\left(X \times_{S} T\right) \hookrightarrow \underline{\operatorname{Coh}}_{/ S}^{S^{\prime}}(X) \quad \text { et } \quad\left(\operatorname{id}_{X^{\prime}} \times t^{\prime}\right)_{*}: \underline{\mathrm{Coh}}_{/ T^{\prime}}\left(X^{\prime} \times_{S^{\prime}} T^{\prime}\right) \hookrightarrow \underline{\mathrm{Coh}}_{/ S^{\prime}}\left(X^{\prime}\right)
$$

avec $X \in \mathrm{Sm} / S$ et $X^{\prime} \in \mathrm{Sm} / S^{\prime}$. Les flèches horizontales sont induites par les foncteurs images inverses

$$
\left(\operatorname{id}_{Y} \times s^{\prime}\right)^{*}: \underline{\operatorname{Coh}}_{/ T}^{T^{\prime}}(Y) \longrightarrow \underline{\operatorname{Coh}}_{/ T^{\prime}}\left(Y \times_{T} T^{\prime}\right) \text { et }\left(\operatorname{id}_{X} \times s\right)^{*}: \underline{\operatorname{Coh}}_{/ S}^{S^{\prime}}(X) \longrightarrow \underline{\operatorname{Coh}}_{/ S^{\prime}}\left(X \times_{S} S^{\prime}\right)
$$

avec $Y \in \mathrm{Sm} / T$ et $X \in \mathrm{Sm} / S$.

En utilisant les adjonctions $\left(t_{*}, t^{!}\right)$et $\left(t_{*}^{\prime}, t^{\prime}\right)$, on obtient un diagramme commutatif

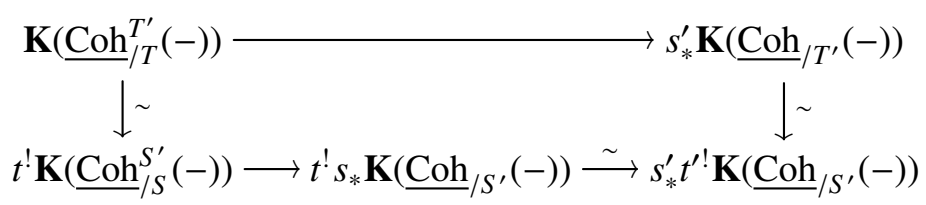


où les flèches verticales sont inversibles d'après le Lemme 7.14. En utilisant les adjonctions $\left(s^{*}, s_{*}\right)$ et $\left(s^{\prime *}, s_{*}^{\prime}\right)$, on obtient le diagramme commutatif

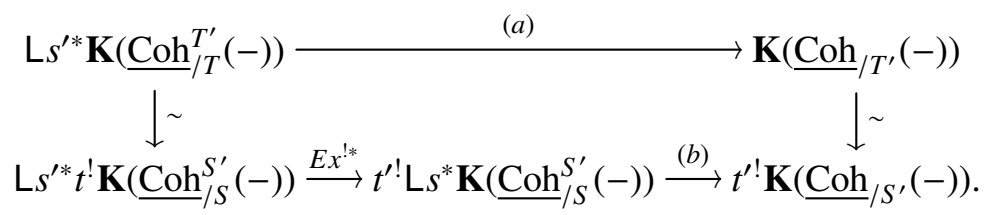

Le Corollaire 7.12 et la construction des foncteurs « image inverse » montrent que les morphismes $(a)$ et $(b)$ ci-dessus sont inversibles. Il vient que le morphisme $E x^{! *}$ ci-dessus est inversible. Ceci termine la preuve du théorème.

Dans la suite, nous notons $\mathbf{B G L}_{S}$ un objet dans $\mathbf{S H}(S)$ qui représente la $K$-théorie algébrique des schémas lisses au-dessus d'une base régulière $S$, i.e., qui est isomorphe à $\mathbf{K}\left(\underline{\mathrm{Coh}}_{S}(-)\right)$. On pourra prendre par exemple le $T$-spectre introduit par Voevodsky (cf. [50, §6.2]).

D'après l'Annexe $\mathrm{A}$, on dispose d'un foncteur de localisation $\mathbf{S H}(S) \longrightarrow \mathbf{S H}_{\mathbb{Q}}(-)$ et d'une équivalence de catégories $\mathrm{T} \circ \mathrm{N}(-\otimes \mathbb{Q}): \mathbf{S H}_{\mathbb{Q}}(S) \simeq \mathbf{D A}(S, \mathbb{Q})($ cf. $(165))$.

Corollaire 7.17 - Soit $S$ un schéma régulier. Alors $\mathrm{T} \circ \mathrm{N}\left(\mathbf{B G L} \mathbf{L}_{S} \otimes \mathbb{Q}\right) \in \mathbf{D A}(S, \mathbb{Q})$ est pur relativement à tout carré transversal d'immersions fermées $(C)$ comme dans le Théorème 7.4.

Demonstration Ceci découle immédiatement du Théorème 7.16 et du Corollaire A.14.

Le résultat qui suit est une reformulation d'un fait bien connu, à savoir que la $K$-théorie étale et la $K$-théorie algébrique coïncident à torsion près.

Lemme 7.18 - Soit $S$ un schéma régulier. L'objet $\mathrm{T} \circ \mathrm{N}\left(\mathbf{B G L}_{S} \otimes \mathbb{Q}\right) \in \mathbf{D A}(S, \mathbb{Q})$ est ét-local.

Demonstration Il s'agit de montrer que le complexe de préfaisceaux de $\mathbb{Q}$-espaces vectoriels $K=\operatorname{REv}_{n}\left(\mathrm{~T} \circ \mathrm{N}\left(\mathbf{B G L}_{S} \otimes \mathbb{Q}\right)\right)$ est ét-local pour tout $n \in \mathbb{N}$. Autrement dit, il faut montrer que $\mathrm{H}^{i}(K(X)) \simeq \mathrm{H}_{\mathrm{et}}^{i}(X, K)$ pour tout $X \in \mathrm{Sm} / S$. Puisque $K$ est Nis-local, il suffira de montrer que $\mathrm{H}_{\mathrm{Nis}}^{i}(X, K) \simeq \mathrm{H}_{\text {ét }}^{i}(X, K)$. Le foncteur d'oubli oét $: \mathbf{S h}_{\text {ét }}(\mathrm{Et} / X, \mathbb{Q}) \longrightarrow \mathbf{S h}_{\mathrm{Nis}}(\mathrm{Et} / X, \mathbb{Q})$ est exact. Il suffit alors de montrer que le morphisme canonique $K \longrightarrow$ oétát $(K)$ est un quasi-isomorphisme. Ceci se vérifie sur les schémas henséliens. On est alors ramené à montrer la propriété suivante. $\mathrm{Si}$ $e: X^{\prime} \longrightarrow X$ est un revêtement étale galoisien (entre $S$-schémas lisses et connexes) de groupe de Galois $G$, alors $K(X) \longrightarrow K\left(X^{\prime}\right)^{G}$ est un quasi-isomorphisme. Ceci est équivalent à montrer que pour tout $i \in \mathbb{N}, \mathrm{K}_{i}(X) \otimes \mathbb{Q} \longrightarrow\left(\mathrm{K}_{i}\left(X^{\prime}\right) \otimes \mathbb{Q}\right)^{G}$ est inversible. (On utilise ici la Proposition A.13 et le fait que $\mathrm{T} \circ \mathrm{N}: \mathbf{S H}_{\mathbb{Q}}(S) \longrightarrow \mathbf{D A}(S, \mathbb{Q})$ est un équivalence de catégories.) Cette propriété découle aussitôt de l'existence et des propriétés des transferts en $K$-théorie algébrique (cf. [38, §7]).

Corollaire 7.19 - Soit $S$ un schéma régulier. Alors $\mathrm{T} \circ \mathrm{N}\left(\mathbf{B G L}_{S} \otimes \mathbb{Q}\right) \in \mathbf{D A}^{\mathrm{e} t}(S, \mathbb{Q})$ est pur relativement à tout carré transversal d'immersions fermées $(C)$ comme dans le Théorème 7.4.

Demonstration Notons aét : DA $(S, \mathbb{Q}) \longrightarrow \mathbf{D A}^{\text {ét }}(S, \mathbb{Q})$ le foncteur de localisation, donné par l'identité sur les objets, et Roét son adjoint à droite. Posons $\mathbf{E}_{S}=\mathrm{T} \circ \mathrm{N}\left(\mathbf{B G L} \mathbf{L}_{S} \otimes \mathbb{Q}\right) \in \mathbf{D A}(S, \mathbb{Q})$. Vus le Corollaire 7.17 et le fait que aét commute aux foncteurs " image inverse », il suffit de montrer que les morphismes canoniques

$$
\mathrm{a}_{\text {êt } t} t^{\prime} \mathbf{E}_{S} \longrightarrow t^{\prime} \mathrm{a} e ́ t^{\mathbf{E}_{S}} \quad \text { et } \quad \mathrm{a}_{\text {ét }} t^{\prime} \mathbf{E}_{S^{\prime}} \longrightarrow t^{\prime} \mathrm{a}_{\mathrm{ét}} \mathbf{E}_{S^{\prime}}
$$

sont inversibles. (Utiliser l'isomorphisme $s^{*} \mathbf{E}_{S} \simeq \mathbf{E}_{S^{\prime}}$ déduit du Corollaire 7.12.) Il suffit bien entendu de se concentrer sur le premier morphisme. D'après le Lemme 7.18, $\mathbf{E}_{S}$ est ét-local. Il s'ensuit que $\mathbf{E}_{S} \longrightarrow$ Roétaét $^{\mathbf{E}_{S}}$ est un isomorphisme. En appliquant $t^{!}$et en utilisant la commutation $t^{!} \circ$ Roét $\simeq$ Roét $\circ t^{\prime}$, on déduit un isomorphisme canonique $t^{!} \mathbf{E}_{S} \simeq$ Roét $t^{\prime} \mathrm{a}_{\text {ét }} \mathbf{E}_{S}$. En appliquant aét et en utilisant que le morphisme de counité aét Roét $\longrightarrow$ id est inversible, on déduit un isomorphisme canonique aét $t \mathbf{E}_{S} \simeq t^{!} \mathrm{a} e ́ t \mathbf{E}_{S}$. On laissera au lecteur le soin de vérifier que cet isomorphisme coïncide avec le morphisme qui nous intéresse.

Le Théorème 7.4 découle maintenant du fait bien connu suivant. 
Proposition 7.20 - Soit $S$ un schéma régulier de dimension de Krull finie. Dans $\mathbf{D A}{ }^{\mathrm{e}}(S, \mathbb{Q})$, l'objet unité $\mathbb{Q}_{S}(0)$ est un facteur direct de $\mathrm{T} \circ \mathrm{N}\left(\mathbf{B G} \mathbf{L}_{S} \otimes \mathbb{Q}\right)$.

Demonstration Il y a plusieurs façons de procéder. Nous prendrons une voie rapide et facile qui repose sur le modèle de BGL fourni par [15] et [45]. Rappelons de quoi il s'agit. Pour $n \in \mathbb{N}$, on a une inclusion canonique $\mathbb{P}_{S}^{n} \hookrightarrow \mathbb{P}_{S}^{n+1}$ donnée par $\left[x_{0}: \cdots: x_{n}\right] \rightsquigarrow\left[x_{0}: \cdots: x_{n}: 0\right]$. On note $\mathbb{P}_{S}^{\infty}$ la colimite des $\mathbb{P}_{S}^{n}$ dans la catégorie des préfaisceaux sur $\mathrm{Sm} / S$. Les plongements de Segre $\mathbb{P}_{S}^{m} \times_{S} \mathbb{P}_{S}^{n} \longrightarrow \mathbb{P}_{S}^{m n+m+n}$ fournissent un morphisme $m: \mathbb{P}_{S}^{\infty} \times_{S} \mathbb{P}_{S}^{\infty} \longrightarrow \mathbb{P}_{S}^{\infty}$. Considérons le $T$-spectre symétrique $\mathbf{X}=\operatorname{Sus}_{T, \Sigma}^{0}\left(\mathbb{P}_{S}^{\infty}\right)$. Le morphisme $m$ induit un morphisme $m: \mathbf{X} \otimes \mathbf{X} \longrightarrow \mathbf{X}$ dans $\mathbf{S H}(S)$. Par ailleurs, on dispose d'un morphisme $\beta: \mathbb{1}(1)[2] \longrightarrow \mathbf{X}$, appelé le morphisme de Bott. Il est donné par la composition de

$$
\mathbb{1}(1)[2] \longrightarrow \operatorname{Sus}_{T, \Sigma}^{0}\left(\mathbb{P}_{S}^{1}\right) \longrightarrow \operatorname{Sus}_{T, \Sigma}^{0}\left(\mathbb{P}_{S}^{\infty}\right) .
$$

La première fléche est induite par la décomposition $\operatorname{Sus}_{T, \Sigma}^{0}\left(\mathbb{P}_{S}^{1}\right) \simeq \mathbb{1} \bigoplus \mathbb{1}(1)[2]$ du motif de $\mathbb{P}_{S}^{1}$ et la seconde flèche est induite par l'inclusion évidente $\mathbb{P}_{S}^{1} \hookrightarrow \mathbb{P}_{S}^{\infty}$. On peut alors former la $\mathbb{N}$-suite

$$
\{\mathbf{X} \longrightarrow \mathbf{X}(-1)[-2] \longrightarrow \cdots \longrightarrow \mathbf{X}(-n)[-2 n] \longrightarrow \mathbf{X}(-n-1)[-2 n-2] \longrightarrow \cdots\}
$$

où la $n$-ième flèche est donnée par la composition de

$$
\mathbf{X}(-n+1)[-2 n+2] \simeq(\mathbf{X} \otimes \mathbb{1}(1)[2])(-n)[-2 n] \stackrel{\beta}{\longrightarrow}(\mathbf{X} \otimes \mathbf{X})(-n)[-2 n] \stackrel{m}{\longrightarrow} \mathbf{X}(-n)[-2 n] .
$$

La colimite homotopique (dans $\mathbf{S H}(S)$ ) de (75) sera notée $\mathbf{X}\left[\beta^{-1}\right]$. Avec ces notations, un résultat principal de [15] et [45] affirme qu'on a un isomorphisme $\mathbf{X}\left[\beta^{-1}\right] \simeq \mathbf{B G L}_{S}$ dans $\mathbf{S H}(S)$.

Expliquons comment conclure. Il faut prouver que $\mathbb{Q}_{S}(0)$ est un facteur direct de $\mathrm{T} \circ \mathrm{N}\left(\mathbf{X}\left[\beta^{-1}\right] \otimes\right.$ $\mathbb{Q})$ dans $\mathbf{D A}{ }^{\mathrm{e}}(S, \mathbb{Q})$. Or, on a un isomorphisme canonique

$$
\mathrm{T} \circ \mathrm{N}(\mathbf{X} \otimes \mathbb{Q}) \simeq \operatorname{colim}_{n \in \mathbb{N}} \operatorname{Sus}_{T, \Sigma}^{0}\left(\mathbb{P}_{S}^{n} \otimes \mathbb{Q}\right) .
$$

Le Lemme 7.21 ci-dessous, fournit un isomorphisme canonique $\mathrm{T} \circ \mathrm{N}(\mathbf{X} \otimes \mathbb{Q}) \simeq \bigoplus_{n \in \mathbb{N}} \mathbb{Q}_{S}(n)[2 n]$ dans $\mathbf{D A}^{\mathrm{e}}{ }^{\mathrm{e}}(S, \mathbb{Q})$. La partie (b) du même lemme montre que la multiplication sur $\mathrm{T} \circ \mathrm{N}(\mathbf{X} \otimes \mathbb{Q})$ correspond à la structure d'algèbre naturelle sur $\bigoplus_{n \in \mathbb{N}} \mathbb{Q}_{S}(n)[2 n]$. Enfin, il est clair que le morphisme de Bott identifie $\mathbb{Q}_{S}(1)[2]$ avec le second facteur de $\bigoplus_{n \in \mathbb{N}} \mathbb{Q}_{S}(n)[2 n]$. Il s'ensuit immédiatement que $\mathrm{T} \circ \mathrm{N}\left(\mathbf{X}\left[\beta^{-1}\right] \otimes \mathbb{Q}\right)$ est isomorphe à $\bigoplus_{n \in \mathbb{Z}} \mathbb{Q}_{S}(n)[2 n]$ dans $\mathbf{D A}^{\text {ét }}(S, \mathbb{Q})$. Ceci termine la preuve de la proposition.

Lemme 7.21 - Soient $S$ un schéma de base et $m, n \in \mathbb{N}$. On a une décomposition en somme directe dans $\mathbf{D} \mathbf{A}^{\mathrm{t}}(S, \mathbb{Q})$ :

$$
\operatorname{Sus}_{T, \Sigma}^{0}\left(\mathbb{P}_{S}^{m} \otimes \mathbb{Q}\right) \simeq \bigoplus_{0 \leq i \leq m} \mathbb{Q}_{S}(i)[2 i] .
$$

De plus, modulo cet isomorphisme on a :

(a) Le morphisme $\bigoplus_{0 \leq i \leq m} \mathbb{Q}_{S}(i)[2 i] \longrightarrow \bigoplus_{0 \leq i \leq m+1} \mathbb{Q}_{S}(i)[2 i]$, induit par l'immersion évidente $\mathbb{P}_{S}^{m} \hookrightarrow \mathbb{P}_{S}^{m+1}$, envoie le $i$-ième facteur identiquement sur le i-ième facteur pour tout $0 \leq i \leq m$.

(b) Le morphisme $\bigoplus_{0 \leq i \leq m, 0 \leq j \leq n} \mathbb{Q}_{S}(m+n)[2 m+2 n] \longrightarrow \bigoplus_{0 \leq k \leq m n+m+n} \mathbb{Q}_{S}(k)[2 k]$, induit par le plongement de Segre $\mathbb{P}_{S}^{m} \times \mathbb{P}_{S}^{n} \longrightarrow \mathbb{P}_{S}^{m n+m+n}$, envoie le $(i, j)$-ième facteur identiquement sur le $(i+j)$-ième facteur pour tout $0 \leq i \leq m$ et $0 \leq j \leq n$.

Demonstration Si $\pi_{\mathbb{P}_{S}^{m}}: \mathbb{P}_{S}^{m} \longrightarrow S$ est la projection structurale, on a un couple de foncteurs adjoints $\left(\left(\pi_{\mathbb{P}_{S}^{m}}\right)_{\sharp}\left(\pi_{\mathbb{P}_{S}^{m}}\right)^{*},\left(\pi_{\mathbb{P}_{S}^{m}}\right)_{*}\left(\pi_{\mathbb{P}_{S}^{m}}\right)^{*}\right)$. En notant que $\underline{\mathbf{M}}_{c o h}\left(\mathbb{P}_{S}^{m}\right)=\left(\pi_{\mathbb{P}_{S}^{m}}\right)_{*}\left(\pi_{\mathbb{P}_{S}^{m}}\right)^{*}$, le Corollaire 2.12 fournit alors un 2-isomorphisme

$$
\left(\pi_{\mathbb{P}_{S}^{m}}\right)_{\sharp}\left(\pi_{\mathbb{P}_{S}^{m}}\right)^{*} \simeq \bigoplus_{0 \leq i \leq m} \operatorname{id}_{\mathbf{D A}^{\mathrm{et}}(S, \mathbb{Q})}(i)[2 i] .
$$

Il suffit alors de l'appliquer à $\mathbb{Q}_{S}(0)$ pour obtenir l'isomorphisme (76). 
Pour la suite, nous reprenons le morphisme $f_{m}:\left(\mathbb{P}_{S}^{1}\right)^{m} \longrightarrow \mathbb{P}_{S}^{m}$ (cf. (21)). Par construction, le morphisme

$$
\bigoplus_{I \subset \llbracket 1, m \rrbracket} \mathbb{Q}_{S}(\operatorname{card}(I))[2 \operatorname{card}(I)]=\left(\mathbb{Q}_{S}(0) \oplus \mathbb{Q}_{S}(1)[2]\right)^{\otimes m} \longrightarrow \bigoplus_{0 \leq i \leq m} \mathbb{Q}_{S}(i)[2 i],
$$

induit par $f_{m}$, envoie identiquement le $I$-ième facteur sur le card(I)-ième facteur. L'assertion (a) découle aussitôt. Passons à l'assertion (b). On utilise pour cela le diagramme commutatif

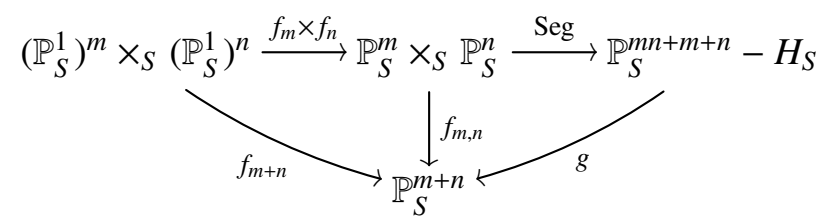

où les morphismes $f_{m, n}$ et $g$ sont donnés sur les coordonnés homogènes par

$$
\begin{gathered}
f_{m, n}\left(\left[x_{0}: \cdots: x_{m}\right],\left[y_{0}: \cdots: y_{n}\right]\right)=\left[\left(\sum_{0 \leq i \leq m, 0 \leq j \leq n, i+j=l} x_{i} y_{j}\right)_{0 \leq l \leq m+n}\right] \\
\text { et } \quad g\left(\left[\left(z_{i j}\right)_{0 \leq i \leq m, 0 \leq j \leq n}\right]\right)=\left[\left(\sum_{0 \leq i \leq m, 0 \leq j \leq n, i+j=l} z_{i j}\right)_{0 \leq l \leq m+n}\right] .
\end{gathered}
$$

Bien entendu $H_{S}$ est le sous-espace linéaire défini par les équations $\sum_{i+j=l} z_{i j}=0$. Il est de codimension $m+n+1$ et n'intersecte pas l'image du plongement de Segre. Le morphisme $g$ est la projection d'un fibré vectoriel. L'invariance par homotopie entraîne alors que l'image du morphisme $\bigoplus_{0 \leq i \leq m, 0 \leq j \leq n} \mathbb{Q}_{S}(m+n)[2 m+2 n] \longrightarrow \bigoplus_{0 \leq k \leq m n+m+n} \mathbb{Q}_{S}(k)[2 k]$, induit par le plongement de Segre, est contenue dans le facteur direct $\bigoplus_{0 \leq l \leq m+n} \mathbb{Q}_{S}(l)[2 l]$. De plus, le morphisme $\bigoplus_{0 \leq i \leq m, 0 \leq j \leq n} \mathbb{Q}_{S}(m+n)[2 m+2 n] \longrightarrow \bigoplus_{0 \leq l \leq m+n} \mathbb{Q}_{S}(l)[2 l]$ ainsi obtenu coïncide avec le morphisme induit par $f_{m, n}$. Le résultat recherché est maintenant clair.

\section{Constructibilité et dualité}

Commençons par rappeler la notion de constructibilité pour les motifs étales.

Definition 8.1 - Soient $S$ un schéma et $\Lambda$ un anneau de coefficients. On note $\mathbf{D} \mathbf{A}_{\mathrm{ct}}^{\mathrm{et}}(S, \Lambda)$ la plus petite sous-catégorie triangulée de $\mathbf{D A}^{\mathrm{e} t}(S, \Lambda)$ stable par facteurs directs et contenant les objets de la forme $\operatorname{Sus}_{T, \Sigma}^{n}(U \otimes \Lambda)$ avec $U \in \mathrm{Sm} / S$ et $n \in \mathbb{N}$. Un $S$-motif étale est dit constructible lorsqu'il appartient à $\mathbf{D A} \mathrm{ct}_{\mathrm{ct}}^{\mathrm{et}}(S, \Lambda)$.

Remarque 8.2 - Si $g: U \longrightarrow S$ est un $S$-morphisme lisse et $n \in \mathbb{N}$, on a un isomorphisme $g_{\sharp}\left(\Lambda_{U}(-n)[-2 n]\right) \simeq \operatorname{Sus}_{T, \Sigma}^{n}(U \otimes \Lambda)$ dans $\mathbf{D A}^{\text {ét }}(S, \Lambda)$. Ainsi, la notion de constructibilité introduite dans la Définition 8.1 est un cas particulier de celle de $\lambda$-constructibilité de [4, Déf. 2.2.3]; on la retrouve en prenant pour $\lambda$ le sous-ensemble $\left\{\Lambda_{S}(-n) ; n \in \mathbb{N}\right\} \subset \mathbf{D A}^{\text {ét }}(S, \Lambda)$.

Proposition 8.3 - Soient $S$ un schéma et $\Lambda$ un anneau de coefficients. On suppose que l'Hypothèse 3.17 est satisfaite. Alors, un objet de $\mathbf{D A}^{\mathrm{e}}{ }^{\mathrm{t}}(S, \Lambda)$ est constructible si et seulement si il est compact.

Demonstration Ceci découle de la Proposition 3.19 jointe à [4, Prop. 2.1.24].

On note le corollaire suivant qui nous sera utile plus tard.

CorollaIRe 8.4 - Soient $S$ un schéma et $\Lambda$ un anneau de coefficients qui satisfont à l'Hypothèse 3.17. Soit $M \in \mathbf{D A}^{\mathrm{e}}(S, \Lambda)$ et supposons que pour tout nombre premier $\ell$ inversible sur $S$ il existe un entier e premier à $\ell$ tel que $M \otimes_{\Lambda} \Lambda\left[e^{-1}\right] \in \mathbf{D A}^{\mathrm{e}^{\mathrm{t}}}\left(S, \Lambda\left[e^{-1}\right]\right)$ est constructible. Alors $M$ est constructible.

Demonstration On peut reformuler la condition de l'énoncé est disant qu'il existe une famille finie d'entiers naturels $\left(e_{i}\right)_{1 \leq i \leq n}$ qui engendre $\Lambda$ comme idéal et telle que $M\left[e_{i}^{-1}\right]=M \otimes_{\Lambda} \Lambda\left[e_{i}^{-1}\right] \in$ 
$\mathbf{D A}^{\text {ét }}\left(S, \Lambda\left[e_{i}^{-1}\right]\right)$ est constructible pour tout $1 \leq i \leq n$. Vue la Proposition 8.3, il s'agit de montrer que $M$ est compact en sachant que les $M\left[e_{i}^{-1}\right]$ sont compacts.

Soit $\left(A_{\alpha}\right)_{\alpha \in I}$ une famille d'objets de $\mathbf{D} \mathbf{A}^{\text {ét }}(S, \Lambda)$. Il faut montrer que l'homomorphisme évident

$$
\bigoplus_{\alpha \in I} \operatorname{hom}_{\mathbf{D A}^{\mathrm{et}}(S, \Lambda)}\left(M, A_{\alpha}[r]\right) \longrightarrow \operatorname{hom}_{\mathbf{D A}^{\mathrm{et}}(S, \Lambda)}\left(M, \bigoplus_{\alpha \in I} A_{\alpha}[r]\right)
$$

est bijectif pour tout $r \in \mathbb{Z}$. Vus les triangles distingués

$$
A_{\alpha} \longrightarrow A_{\alpha} \otimes_{\mathbb{Z}} \mathbb{Z}\left[e_{i}^{-1}\right] \longrightarrow A_{\alpha} \otimes_{\mathbb{Z}}\left(\mathbb{Z}\left[e_{i}^{-1}\right] / \mathbb{Z}\right) \longrightarrow
$$

il suffit de prouver que les homomorphismes (77) sont bijectifs pour les familles $\left(A_{\alpha} \otimes_{\mathbb{Z}} \mathbb{Z}\left[e_{i}^{-1}\right]\right)_{\alpha \in I}$ et $\left(A_{\alpha} \otimes_{\mathbb{Z}}\left(\mathbb{Z}\left[e_{i}^{-1}\right] / \mathbb{Z}\right)\right)_{\alpha \in I}$ (lorsque $\left.1 \leq i \leq n\right)$. Or, $\mathbb{Z}\left[e_{i}^{-1}\right] / \mathbb{Z}$ est la somme directe des $\mathbb{Q} / \mathbb{Z}(p)$, où $p$ parcourt l'ensemble (fini) des diviseurs premiers de $e_{i}$, et chaque $\Lambda \otimes_{\mathbb{Z}} \mathbb{Q} / \mathbb{Z}_{(p)}$ est un $\Lambda\left[e_{j}^{-1}\right]$ module pour au moins un $1 \leq j \leq n$. On voit alors qu'il suffit de prouver que l'homomorphisme (77) est une bijection pour les familles $\left(B_{\beta}\right)_{\beta \in J}$ d'objets de $\mathbf{D A}^{\text {ét }}(S, \Lambda)$ provenant d'une famille $\left(B_{\beta}^{\prime}\right)_{\beta \in J}$ d'objets de $\mathbf{D} A^{\text {ét }}\left(S, \Lambda\left[e_{i}^{-1}\right]\right)$ par le foncteur évident $\mathbf{D} A^{\text {ét }}\left(S, \Lambda\left[e_{i}^{-1}\right]\right) \longrightarrow \mathbf{D A}^{\text {ét }}(S, \Lambda)$. Par adjonction, l'homomorphisme (77) s'écrit dans ce cas :

$$
\bigoplus_{\beta \in J} \operatorname{hom}_{\mathbf{D A}^{\epsilon \mathrm{t}}\left(S, \Lambda\left[e_{i}^{-1}\right]\right)}\left(M\left[e_{i}^{-1}\right], B_{\beta}^{\prime}[r]\right) \longrightarrow \operatorname{hom}_{\mathbf{D A}^{\epsilon \mathrm{t}}\left(S, \Lambda\left[e_{i}^{-1}\right]\right)}\left(M\left[e_{i}^{-1}\right], \bigoplus_{\beta \in J} B_{\beta}^{\prime}[r]\right) .
$$

Ce dernier est une bijection puisque $M\left[e_{i}^{-1}\right] \in \mathbf{D A}^{\mathrm{et}}\left(S, \Lambda\left[e_{i}^{-1}\right]\right)$ est supposé compact.

Proposition 8.5 - Soient $S$ un schéma de base et $\Lambda$ un anneau de coefficients. Soit $f: Y \longrightarrow X$ un morphisme de $S$-schémas quasi-projectifs. Alors, les objets constructibles dans $\mathbf{D} \mathbf{A}^{\mathrm{et}}(-, \Lambda)$ sont préservés par:

(a) l'opération $f^{*}$ sans hypothèse supplémentaire sur $f$;

(b) l'opération $f_{*}$ lorsque $f$ est projectif;

(c) l'opération $f_{\text {! }}$ sans hypothèse supplémentaire sur $f$;

(d) l'opération $f^{!}$lorsque $f$ est lisse.

Demonstration Il s'agit d'un cas particulier de [4, Scholie 2.2.34, A et B].

Notre tâche principale dans ce paragraphe est d'expliquer comment le théorème d'uniformisation locale de Gabber [14] (voir aussi [25, Expo. IX, Th. 1.1]) permet de se débarasser des hypothèses « $f$ projectif » et « $f$ lisse » dans la Proposition 8.5, (b) et (d). Rappelons d'abord l'énoncé du théorème d'uniformisation locale de Gabber.

Theoreme 8.6 - (Gabber) Soient $X$ un schéma excellent et $Z \subset X$ une partie fermée partout de codimension non nulle. On fixe un nombre premier $\ell$ inversible sur $X$. Il existe alors une famille finie de diagrammes commutatifs, indexée par $i \in I$,

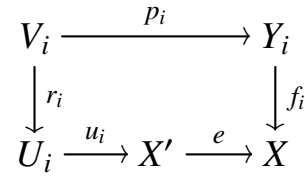

(le morphisme e $: X^{\prime} \longrightarrow X$ étant le même pour tous les diagrammes de la famille) telle que les propriétés suivantes sont satisfaites.

(a) Les schémas $Y_{i}$ sont réguliers et connexes; chaque $Y_{i}$ domine une composante irréductible de $X$.

(b) Les morphismes $f_{i}$ sont génériquement finis et $f_{i}^{-1}(Z)$ est le support d'un diviseur strictement à croisements normaux.

(c) Le morphisme e est une modification, i.e., il est projectif et induit un isomorphisme d'un ouvert dense de $X^{\prime}$ sur un ouvert dense de $X$. 
(d) Les schémas $U_{i}$ sont irréductibles, les morphismes $u_{i}$ sont étales et la famille $\left(U_{i}\right)_{i \in I}$ est un recouvrement Nisnevich de $X^{\prime}$.

(e) Les schémas $V_{i}$ sont irréductibles, et les morphismes $r_{i}$ sont finis, plats et de degré générique premier à $\ell$.

Proposition 8.7 - On suppose que le schéma de base $S$ est excellent et que l'Hypothèse 7.3 est satisfaite. Soient $X$ un $S$-schéma quasi-projectif et $j: X-Z \hookrightarrow X$ l'inclusion de l'ouvert complémentaire d'un fermé $Z \subset X$. Alors, l'opération $j_{*}: \mathbf{D A}^{\mathrm{e} t}(X-Z, \Lambda) \longrightarrow \mathbf{D A}^{\mathrm{e}}(X, \Lambda)$ préserve les objets constructibles.

À quelques détails techniques près, la preuve de cette proposition suit fidèlement la méthode très astucieuse mise au point par Gabber dans sa démonstration du résultat analogue en cohomologie étale (cf. [14, second proof of Th. 0.1], [24, Th. 1] et [25, Expo. XIII, Th. 1.1.1]). Les ingrédients essentiels sont le théorème de pureté absolue (cf. le Théorème 7.4) et le théorème d'uniformisation locale de Gabber (cf. le Théorème 8.6). Par soucis d'exposition, nous reprenons les détails. On commence par une réduction standard.

Lemme 8.8 - Pour démontrer la Proposition 8.7, il suffit de vérifier que $j_{*} \Lambda(0) \in \mathbf{D A}^{\mathrm{e} t}(X, \Lambda)$ est constructible pour tout $S$-schéma quasi-projectif $X$ et tout fermé $Z \subset X$.

Demonstration La Proposition 3.19 et [4, Lem. 2.2.23] entraînent que $\mathbf{D A}^{\text {ét }}(X-Z, \Lambda)$ est compactement engendrée par les motifs de la forme $\pi_{*}^{\prime} \Lambda(n)$ avec $\pi^{\prime}: Y^{\prime} \longrightarrow X-Z$ un morphisme projectif et $n \in \mathbb{Z}$. (Remarquons que ces objets sont constructibles d'après la Proposition 8.5, (b).) Il suffit donc de prouver que $j_{*} \pi_{*}^{\prime} \Lambda(0)$ est constructible.

On fixe une compactification $j^{\prime}: Y^{\prime} \hookrightarrow Y$ au-dessus de $X$ avec $\pi: Y \longrightarrow X$ un $X$-schéma projectif et $Y^{\prime}$ dense dans $Y$. (Puisque $\pi^{\prime}$ est projectif, on a $Y^{\prime}=Y-\pi^{-1}(Z)$ mais ceci est inutile pour la suite.) Vu que $j \circ \pi^{\prime}=\pi \circ j^{\prime}$, il suffit de montrer que $\pi_{*} j_{*}^{\prime} \Lambda(0)$ est constructible. Or, d'après la Proposition 8.5, (b), le foncteur $\pi_{*}$ préserve les objets constructibles. Il est donc suffisant de montrer que $j_{*}^{\prime} \Lambda(0)$ est constructible. Le lemme est démontré.

Le Théorème 7.4 sera utilisé à travers le résultat suivant.

Lemme 8.9 - On suppose que l'Hypothèse 7.3 est satisfaite. Soient X un $S$-schéma quasiprojectif et $j: X-Z \hookrightarrow X$ l'inclusion de l'ouvert complémentaire d'un fermé $Z \subset X$. On suppose que $X$ est régulier et que $Z$ est le support d'un diviseur strictement à croisements normaux. Alors, $j_{*} \Lambda(0) \in \mathbf{D A}^{\mathrm{e} t}(X, \Lambda)$ est constructible.

Demonstration Notons $i: Z \hookrightarrow X$ l'immersion complémentaire à $j$. Vu le 2-triangle distingué de localité (cf. [4, Prop. 1.4.9]), il suffit de montrer que $i ! \Lambda(0)$ est constructible. Notons $Z_{1}, \cdots, Z_{n}$ les composantes irréductibles de $Z$ et posons $Z_{I}=\cap_{i \in I} Z_{i}$ pour $\emptyset \neq I \subset \llbracket 1, n \rrbracket$. Si $u_{I}: Z_{I} \hookrightarrow Z$ est l'inclusion évidente, [4, Lem. 2.2.31] entraîne que $i^{!} \Lambda(0)$ est dans la sous-catégorie triangulée de $\mathbf{D A}^{\text {ét }}(Z, \Lambda)$ engendrée par les $u_{I *}\left(i \circ u_{I}\right)^{!} \Lambda(0)$ pour $\emptyset \neq I \subset \llbracket 1, n \rrbracket$. On est donc ramené à montrer que $s^{!} \Lambda(0)$ est constructible pour l'inclusion $s: Y \hookrightarrow X$ d'un sous-schéma régulier. D'après [4, Cor. 2.2.6], le problème est local. On peut donc supposer que l'idéal de définition de $s$ est globalement engendré par $d$ sections avec $d$ la codimension de $Y$ dans $X$ (supposée constante le long de $Y$ ). On applique enfin le Corollaire 7.5 pour conclure.

On donne maintenant la preuve, suivant la méthode de Gabber, de la Proposition 8.7.

Demonstration On raisonne par induction $\operatorname{sur} \operatorname{krdim}(X)$, la dimension de Krull de $X$. Lorsque cette dimension est nulle, il n'y a rien à prouver. On fixe un entier $d \geq 1$, et on suppose que la Proposition 8.7 est prouvée pour $X \operatorname{lorsque} \operatorname{krdim}(X) \leq d-1$.

Pour $X$ un $S$-schéma quasi-projectif, $Z \subset X$ une partie femée et $n \in \mathbb{N}$, on introduit la propriété $\mathrm{P}(X, Z, n)$ suivante.

$\mathrm{P}(X, Z, n):$ Il existe une partie fermée $T \subset Z$ de codimension plus grande ou égale à $n$ telle que $\left(j_{*} \Lambda(0)\right)_{\mid X-T}$ est constructible.

(Bien entendu, $j$ est l'inclusion de $X-Z$ dans $X$ et $(-)_{\mid X-T}: \mathbf{D A}^{\text {ét }}(X, \Lambda) \longrightarrow \mathbf{D A}^{\text {ét }}(X-T, \Lambda)$ est le foncteur « image inverse » suivant l'inclusion $X-T \hookrightarrow X$.) 
Par la récurrence sur la dimension de Krull de $X$, la propriété $\mathrm{P}(X, Z, n)$ est vraie pour tout $n \in \mathbb{N}$ lorsque $\operatorname{krdim}(X) \leq d-1$. Par ailleurs, la propriété $\mathrm{P}(X, Z, 0)$ est toujours vraie; il suffit de prendre $T=Z$. Pour $n>\operatorname{krdim}(Z)$, la propriété $\mathrm{P}(X, Z, n)$ est équivalente à la constructibilité de $j_{*} \Lambda(0)$. Ainsi, pour démontrer la Proposition 8.7 il suffit de montrer que la proporiété $\mathrm{P}(X, Z, n-1)$ pour tout $S$-schéma quasi-projectif $X$ de dimension de Krull $d$ entraîne la propriété $\mathrm{P}(X, Z, n)$ pour tout $S$-schéma quasi-projectif $X$ de dimension de Krull $d$. On divise la preuve en plusieurs étapes.

Étape $A$ : Fixons un $S$-schéma quasi-projectif $X$ de dimension de Krull $d$ et une partie fermée $Z \subset X$. On ne restreint pas la généralité en supposant que $Z$ est de codimension non nulle. (En effet, on peut remplacer $X$ par l'adhérence de $X-Z$.) Nous cherchons un fermé $T$ de codimension plus grande ou égale à $n$ tel que $\left(j_{*} \Lambda(0)\right)_{\mid X-T}$ soit constructible. Il suffit pour cela de trouver, pour tout nombre premier $\ell$ inversible sur $S$, un entier naturel $e_{\ell} \in \mathbb{N}-\{0\}$ premier à $\ell$ et un fermé $T_{\ell} \subset Z$ de codimension plus grande ou égale à $n$ tels que $\left(j_{*} \Lambda\left[e_{\ell}^{-1}\right](0)\right)_{\mid X-T_{\ell}}$ est constructible dans $\mathbf{D} A^{\text {ét }}\left(X-T_{\ell}, \Lambda\left[e_{\ell}^{-1}\right]\right)$. En effet, si cela était possible, nous obtiendrons alors une famille finie d'entiers naturels $\left(e_{i}\right)_{1 \leq i \leq m}$ qui engendre $\Lambda$ comme idéal et une famille finie de fermés $\left(T_{i}\right)_{1 \leq i \leq m}$ de codimension plus grande ou égale à $n$ dans $Z$ telles que $\left(j_{*} \Lambda\left[e_{i}^{-1}\right](0)\right)_{\mid X-T_{i}}$ est constructible dans $\mathbf{D A}^{\text {ét }}\left(X, \Lambda\left[e_{i}^{-1}\right]\right)$ pour tout $1 \leq i \leq m$. D'après le Corollaire $8.4,\left(j_{*} \Lambda(0)\right)_{X-T}$ est alors constructible dans $\mathbf{D} A^{\text {ét }}(X, \Lambda)$ si nous prenions $T=\cup_{1 \leq i \leq m} T_{i}$.

À partir de maintenant, on fixe un nombre premier $\ell$ inversible sur $S$. On applique le théorème d'uniformisation locale de Gabber à $X$ et $Z$ pour obtenir un diagramme commutatif (79) vérifiant les propriétés (a) à (e) du Théorème 8.6. Appelons $e_{\ell}$ le plus petit multiple commun des degrés génériques des morphismes finis $r_{i}$. C'est un entier premier à $\ell$ et nous allons construire $T \subset Z$ de codimension plus grande ou égale à $n$ tel que $\left(j_{*} \Lambda\left[e_{\ell}^{-1}\right](0)\right)_{\mid X-T}$ est constructible dans $\mathbf{D A}^{\text {ét }}\left(S, \Lambda\left[e_{\ell}^{-1}\right]\right)$. À ce stade, on peut remplacer $\Lambda$ par $\Lambda\left[e_{\ell}^{-1}\right]$ et supposer que les degrés génériques des morphismes finis $r_{i}$ sont inversibles dans $\Lambda$. C'est ce que nous ferions dans la suite.

Étape $B$ : On fixe une fois pour toute un ouvert dense $X^{0} \subset X-Z$. On note $X^{\prime 0}, U_{i}^{0}, V_{i}^{0}$ et $Y_{i}^{0}$ les images inverses de $X^{0}$ dans $X^{\prime}, U_{i}, V_{i}$ et $Y_{i}$ (avec $i \in I$ ). Les inclusions $X^{0} \hookrightarrow X, X^{\prime 0} \hookrightarrow X^{\prime}$, etc, seront désigées par $\iota$. On supposera que la restriction de $e$ à $X^{\prime 0}$ induit un isomorphisme $e^{0}: X^{\prime 0} \simeq X^{0}$. On suppose aussi que pour tout $i \in I$, le morphisme $r_{i}^{0}: V_{i}^{0} \longrightarrow U_{i}^{0}$ est le composé d'un revêtement étale suivi d'une morphisme totalement inséparable.

Notons $D=X-X^{0}$ et $D^{\prime}=X^{\prime}-X^{\prime 0}$. Par l'hypothèse de récurrence appliquée aux couples $(D, Z)$ et $\left(D^{\prime}, Z^{\prime}\right)$, on voit que les cônes des morphismes

$$
j_{*} \iota ! \Lambda(0) \longrightarrow j_{*} \Lambda(0) \quad \text { et } \quad j_{*}^{\prime} \iota ! \Lambda(0) \longrightarrow j_{*}^{\prime} \Lambda(0)
$$

sont constructibles. (On utilise ici le 2-triangle de localité [4, Lem. 1.4.6].) En particulier, la propriété $\mathrm{P}(X, Z, n)$ équivaut à l'existence d'un fermé $T \subset Z$ de codimension plus grande ou égale à $n$ tel que $\left(j_{*} \iota_{!} \Lambda(0)\right)_{\mid X-T}$ soit constructible.

Remarquons aussi que le morphisme canonique

$$
j_{*} \iota ! \Lambda(0) \longrightarrow e_{*} j_{*}^{\prime} \iota ! \Lambda(0)
$$

est un isomorphisme. En effet, $e \circ j^{\prime}=j \circ e^{\prime}$ avec $e^{\prime}: X^{\prime}-Z^{\prime} \longrightarrow X-Z$ le morphisme déduit de $e$. En particulier, $e_{*} j_{*}^{\prime} \simeq j_{*} e_{*}^{\prime}$ et il est suffisant de montrer que le morphisme canonique $\iota ! \Lambda(0) \longrightarrow e_{*}^{\prime} \iota ! \Lambda(0)$ est inversible. Puisque $e^{\prime}$ est projectif, on a un 2 -isomorphisme canonique $e_{!}^{\prime} \simeq e_{*}^{\prime}$ (cf. [4, Th. 1.7.17]). Il en découle une chaîne d'isomorphismes canoniques

$$
e_{*}^{\prime} \iota ! \Lambda(0) \simeq e_{!}^{\prime} \iota ! \Lambda(0) \simeq \iota ! e_{!}^{0} \Lambda(0) \simeq \iota ! \Lambda(0) .
$$

(Rappelons que $e^{0}$ est un isomorphisme entre $X^{\prime 0}$ et $X^{0}$.) Étant donné qu'un endomorphisme de $\iota ! \Lambda(0)$ est inversible si et seulement si sa restriction à $X^{0}$ est inversible, on est ramené à montrer que le morphisme canonique $\iota^{*} \iota ! \Lambda(0) \longrightarrow \iota^{*} e_{*}^{\prime} \iota ! \Lambda(0)$ est inversible. Ceci est clair puisque les deux membres sont canoniquement isomorphes à $\Lambda(0)$.

Étape $C$ : Le but de cette étape est d'introduire quelques notations. La propriété $\mathrm{P}\left(X^{\prime}, Z^{\prime}, n-1\right)$ fournit un fermé $F^{\prime} \subset Z^{\prime}$ de codimension plus grande ou égale à $n-1$ tel que $\left(j_{*}^{\prime} \Lambda(0)\right)_{\mid X^{\prime}-F^{\prime}}$ est 
constructible. Vu que le cône du morphisme de droite dans (80) est constructible, on déduit que $\left(j_{*}^{\prime} \iota ! \Lambda(0)\right)_{\mid X^{\prime}-F^{\prime}}$ est constructible. On pose $F=e\left(F^{\prime}\right)$. La Proposition 8.5 , (b) entraîne aussitôt que $\left(e_{*} j_{*}^{\prime} \iota ! \Lambda(0)\right)_{\mid X-F}$ est constructible. Vu que (81) est inversible, il en est de même de $\left(j_{*} \iota ! \Lambda(0)\right)_{\mid X-F}$.

En utilisant le 2-triangle distingué de localité de [4, Lem. 1.4.6], on voit que pour établir $\mathrm{P}(X, Z, n)$, il faut et il suffit de trouver un ouvert $F^{0} \subset F$ dont le complémentaire est de codimension plus grande ou égale à $n$ dans $Z$ et tel que $\left(j_{*} \iota ! \Lambda(0)\right)_{\mid F^{0}}$ est constructible. (Ici encore, nous avons noté $(-)_{\mid F^{0}}$ le foncteur « image inverse » suivant l'inclusion $F^{0} \hookrightarrow X$.) En effet, le fermé $T=F-F^{0}$ conviendra alors. Ceci montre qu'on peut remplacer $X$ par un voisinage ouvert d'un point générique d'une composante irréductible de $F$ de codimension $n$ dans $Z$. (Bien entendu, tous les autres $X$-schémas en jeu seront remplacés par l'image inverse de ce voisinage.)

En choisissant ce voisinage suffisamment petit, on peut supposer que $F$ est irréductible, que $F^{\prime}$ est l'union disjointe de ses composantes irréductibles et que chacune de ces composantes irréductibles est finie et surjective sur $F$. On peut aussi supposer que pour chaque $i \in I$ il existe un fermé $H_{i} \subset U_{i}$ tel que les $u_{i}$ induisent un isomorphisme $\tilde{u}: \coprod_{i \in I} H_{i} \stackrel{\sim}{\longrightarrow} F^{\prime}$. Pour $i \in I$, on pose alors $G_{i}=r_{i}^{-1}\left(H_{i}\right)$.

Étape $D$ : Notons $b^{\prime}: F^{\prime} \hookrightarrow X^{\prime}$ l'inclusion évidente. D'après les choix de l'étape $\mathrm{C}$, le cône du morphisme $j_{*}^{\prime} \iota ! \Lambda(0) \longrightarrow b_{*}^{\prime} b^{\prime *} j_{*}^{\prime} \iota ! \Lambda(0)$ est constructible. D'après la Proposition 8.5 , (b), il en est du même du cône du morphisme

$$
e_{*} j_{*}^{\prime} \iota_{!} \Lambda(0) \longrightarrow e_{*} b_{*}^{\prime} b^{\prime *} j_{*}^{\prime} \iota_{!} \Lambda(0)
$$

Notons $U=\bigsqcup_{i \in I} U_{i}, H=\bigsqcup_{i \in I} H_{i}, u: U \longrightarrow X^{\prime}$ le morphisme déduit des $u_{i}$ et $c: H \hookrightarrow$ $U$ l'inclusion évidente. Notons aussi $j^{\prime \prime}$ le changement de base de l'inclusion $j: X-Z \hookrightarrow X$ suivant le morphisme $U \longrightarrow X$. Puisque $\tilde{u}$ est un isomorphisme et que $u$ est étale, on a une chaîne d'isomorphismes canoniques

$$
b^{\prime *} j_{*}^{\prime}\left\lfloor! \Lambda(0) \simeq \tilde{u}_{*} \tilde{u}^{*} b^{\prime *} j_{*}^{\prime} \iota ! \Lambda(0) \simeq \tilde{u}_{*} c^{*} u^{*} j_{*}^{\prime} \iota ! \Lambda(0) \simeq \tilde{u}_{*} c^{*} j_{*}^{\prime \prime} \iota ! \Lambda(0) .\right.
$$

Il s'ensuit un isomorphisme canonique

$$
e_{*} b_{*}^{\prime} b^{* *} j_{*}^{\prime} \iota ! \Lambda(0) \simeq e_{*} u_{*} c_{*} c^{*} j_{*}^{\prime \prime} \iota ! \Lambda(0) .
$$

Par ailleurs, si $i \in I$, le morphisme canonique $\iota ! \Lambda(0) \longrightarrow\left(r_{i}\right)_{*} \iota ! \Lambda(0)$ admet une rétraction. En effet, il s'identifie avec le morphisme $\Lambda(0) \longrightarrow\left(r_{i}^{0}\right)_{*} \Lambda(0)$ auquel on applique $\iota$ ! Or, le morphisme $r_{i}^{0}$ est le composé d'un revêtement étale de degré inversible dans $\Lambda$ et d'un morphisme totalement inséparable. Le résultat recherché découle maintenant de [4, Lem. 2.1.165]. Posons $V=\bigsqcup_{i \in I} V_{i}$, $G=\bigsqcup_{i \in I} G_{i}, r: V \longrightarrow U$ et $\tilde{r}: G \longrightarrow H$ les morphismes déduits des $r_{i}, d: G \hookrightarrow V$ l'inclusion évidente et $j^{\prime \prime \prime}$ le changement de base de $j: X-Z \hookrightarrow X$ suivant le morphisme $V \longrightarrow X$. D'après ce que l'on vient de dire, le morphisme $j_{*}^{\prime \prime} \iota ! \Lambda(0) \longrightarrow r_{*} j_{*}^{\prime \prime \prime} \iota ! \Lambda(0)$ admet une rétraction. $\mathrm{Vu}$ l'isomorphisme de changement de base $c^{*} r_{*} \simeq \tilde{r}_{*} d^{*}$, on déduit aussitôt que le morphisme $c_{*} c^{*} j_{*}^{\prime \prime} \iota ! \Lambda(0) \longrightarrow r_{*} d_{*} d^{*} j_{*}^{\prime \prime \prime} \iota ! \Lambda(0)$ admet une rétraction. Au final, on voit que le morphisme canonique

$$
e_{*} u_{*} c_{*} c^{*} j_{*}^{\prime \prime} \iota ! \Lambda(0) \longrightarrow e_{*} u_{*} r_{*} d_{*} d^{*} j_{*}^{\prime \prime \prime} \iota ! \Lambda(0)
$$

admet une rétraction.

Étape $E$ : Finissons la démontration. Nous allons montrer que $j_{*} \iota ! \Lambda(0) \in \mathbf{D A}^{\text {ét }}(X, \Lambda)$ est constructible. À l'instar de [24], nous nous placerons dans le quotient de $\operatorname{Verdier} \mathcal{V}$ de $\mathbf{D A}{ }^{\text {ét }}(X, \Lambda)$ par sa sous-catégorie triangulée $\mathbf{D A}_{\mathrm{ct}}^{\text {et }}(X, \Lambda)$ formée des objets constructibles. Dans $\mathcal{V}$, on a les isomorphismes (81), (82) et (83) et un monomorphisme scindé (84). En les composant, on voit que le morphisme canonique

$$
j_{*} \iota ! \Lambda \longrightarrow e_{*} u_{*} r_{*} d_{*} d^{*} j_{*}^{\prime \prime \prime} \iota ! \Lambda(0)
$$

admet une rétraction dans $\mathcal{V}$. Il suffit donc de prouver que la flèche (85) est nulle dans $\mathcal{V}$.

Or, en utilisant le diagramme commutatif (79), on voit que la flèche (85) se factorise à travers $f_{*} p_{*} d_{*} d^{*} p^{*}\left(j_{Y}\right)_{*} ! \Lambda(0)$. (Ici, on a noté $Y=\bigsqcup_{i \in I} Y_{i}, f: Y \longrightarrow X$ le morphisme déduit des $f_{i}, p: V \longrightarrow Y$ le morphisme déduit des $p_{i}$ et $j_{Y}$ le changement de base de $j: X-Z \hookrightarrow X$ 
JOSEPH AYOUB

suivant le morphisme $Y \rightarrow X$.) Il suffit donc de montrer que cet objet est nul dans $\mathcal{V}$, i.e., qu'il est constructible. Comme dans l'étape $B$, l'hypothèse de récurrence sur la dimension de Krull entraîne que le cône du morphisme $\left(j_{Y}\right)_{*} \iota_{!} \Lambda(0) \longrightarrow\left(j_{Y}\right)_{*} \Lambda(0)$ est constructible. D'autre part, d'après le Lemme $8.9, d^{*} p^{*}\left(j_{Y}\right)_{*} \Lambda(0)$ est constructible. Il en est donc du même de $d^{*} p^{*}\left(j_{Y}\right)_{*} \iota_{!} \Lambda(0)$. Enfin, remarquons que $f \circ p \circ d: H \longrightarrow X$ est un morphisme fini. Il vient que $f_{*} p_{*} d_{*} d^{*} p^{*}\left(j_{Y}\right)_{*} \iota ! \Lambda(0)$ est constructible $d$ 'après la Proposition 8.5 , (b). Ceci termine la preuve.

On obtient maintenant facilement le résultat suivant.

Theoreme 8.10 - On suppose que le schéma de base $S$ est excellent et que l'Hypothèse 7.3 est satisfaite. Soit $f: Y \longrightarrow X$ un morphisme de $S$-schémas quasi-projectifs. Alors, les objets constructibles dans $\mathbf{D A}{ }^{\mathrm{e} t}(-, \Lambda)$ sont préservés par les opérations $f^{*}, f_{*}, f_{!}$et $f^{!}$.

Demonstration Vue la Proposition 8.5, il reste à montrer que

$-j_{*}$ préserve les objets constructibles lorsque $j$ est une immersion ouverte;

- $i^{!}$préserve les objets constructibles lorsque $i$ une immersion fermée.

Lorsque $i$ et $j$ sont complémentaires, le 2-triangle distingué de localité [4, Prop. 1.4.9] montre que les deux propriétés ci-dessus sont équivalentes. Par ailleurs, le cas d'une immersion ouverte est assuré par la Proposition 8.7.

Remarque 8.11 — Lorsque $S$ est un schéma excellent de caractéristiques résiduelles nulles, on dispose de la résolution des singularités par éclatements d'après Hironaka [19]. Dans ce cas, le Théorème 8.10 est une conséquence directe de [4, Scholie 2.2.34] et du Théorème 7.4 qu'on peut alors prouver sans recours à la $K$-théorie algébrique.

On a aussi le résultat suivant.

Theoreme 8.12 - On suppose que le schéma de base $S$ est excellent et que l'Hypothèse 7.3 est satisfaite. Soient $X$ un $S$-schéma quasi-projectif, et A et $B$ deux objets constructibles dans $\mathbf{D A}^{\mathrm{ét}}(X, \Lambda)$. Alors, les objets $A \otimes B$ et $\underline{\operatorname{Hom}}(A, B)$ sont aussi constructibles.

Demonstration Le cas du produit tensoriel est immédiat (cf. [4, Prop. 2.3.60]). On se concentre sur le bifoncteur $\underline{\operatorname{Hom}}(-,-)$. Il suffit de traiter le cas où $A=f_{\sharp} \Lambda(n)$ avec $n \in \mathbb{Z}$ et $f: U \longrightarrow X$ un $S$-morphisme lisse. Or, d'après la formule de projection [4, Prop. 2.3.52] on a des isomorphismes canoniques $\underline{\operatorname{Hom}}\left(f_{\sharp} \Lambda(n), B\right) \simeq f_{*} \underline{\operatorname{Hom}}\left(\Lambda(n), f^{*} B\right) \simeq f_{*} f^{*} B(-n)$. Le Théorème 8.10 permet maintenant de conclure.

La définition suivante est un cas particulier de [4, Def. 2.3.66].

Definition 8.13 - Soit $S$ un schéma de base. Un objet $R \in \mathbf{D A}^{\text {ét }}(S, \Lambda)$ est dit dualisant $s$ 'il est constructible et si le morphisme canonique $M \longrightarrow \underline{\operatorname{Hom}}(\operatorname{Hom}(M, R), R)$ est inversible pour tout objet constructible $M \in \mathbf{D A}^{\mathrm{e}}(S, \Lambda)$.

Le résultat suivant est un cas particulier de [4, Th. 2.3.73]; nous l'incluons pour mémoire. Notons aussi que les méthodes introduites par Gabber dans ses travaux récents sur la cohomologie étale (voir notamment [25, Expo. XVII]) devraient nous permettre de se débarasser de certaines hypothèses encombrantes. Ainsi, le résultat ci-dessous devrait étre vrai pour un schéma de base excellent $S$ muni d'une fonction de dimension (au sens de [25, Expo. XVII]) et un anneau de coefficients $\Lambda$ tels que l'Hypothèse 7.3 est satisfaite.

Theoreme 8.14 - Soient $S$ un schéma de base et $\Lambda$ un anneau de coefficients. On suppose que l'une des deux alternatives ci-dessous est satisfaite.

- S est régulier, excellent et de caractéristiques résiduelles nulles. Sa dimension de Krull est finie et les p-dimensions cohomologiques ponctuelles de $S$ sont uniformément bornées lorsque $p$ parcourt l'ensemble des nombres premiers non inversibles dans $\Lambda$.

- S est un trait excellent et $\Lambda$ est une $\mathbb{Q}$-algèbre.

Alors, on a les propriétés suivantes.

(a) Soit $X$ un $S$-schéma quasi-projectif avec $X$ régulier. Alors, l'objet $\Lambda_{X}(0) \in \mathbf{D A}^{\mathrm{e} t}(X, \Lambda)$ est dualisant.

(b) Pour tout $S$-schéma quasi-projectif $a: X \longrightarrow S$, l'objet $a ! \Lambda_{S}(0) \in \mathbf{D A}^{\mathrm{e} t}(X, \Lambda)$ est dualisant. 
(c) Soient $f: Y \longrightarrow X$ un morphisme de $S$-schémas quasi-projectifs et $R \in \mathbf{D A}^{\mathrm{e}} \mathrm{e}(X, \Lambda)$ un objet dualisant. Alors, l'objet $f^{!} R \in \mathbf{D A}^{\text {ét }}(Y, \Lambda)$ est dualisant.

\section{La réalisation étale des motifs étales (suite)}

Dans cette section, on étend la réalisation étale construite dans la Section 5 aux motifs constructibles à coefficients rationnels. On commence par des préliminaires catégoriques.

Étant donnée une catégorie additive $\mathcal{C}$, on note $\mathcal{C} \otimes \mathbb{Q}$ la catégorie ayant pour objets ceux de $\mathcal{C}$ et telle que $\operatorname{hom}_{\mathcal{C} \otimes \mathbb{Q}}(A, B)=\operatorname{hom}_{\mathcal{C}}(A, B) \otimes_{\mathbb{Z}} \mathbb{Q}$ pour tout $A, B \in \mathcal{C}$. La composition dans $\mathcal{C} \otimes \mathbb{Q}$ est déduite de celle de $\mathcal{C}$ par linéarité. La catégorie $\mathcal{Q} \otimes \mathbb{Q}$ est additive et on a un foncteur additif évident $\mathcal{C} \longrightarrow \mathcal{C} \otimes \mathbb{Q}$ qui est un isomorphisme de catégories si $\mathcal{C}$ est $\mathbb{Q}$-linéaire. Le résultat suivant est certainement bien connu.

Proposition 9.1 - Soit $\mathcal{T}$ une catégorie triangulée. On note $\mathcal{T}_{\text {tor }}$ la sous-catégorie pleine de $\mathcal{T}$ formée des objets $A \in \mathcal{T}$ tels que $n \cdot \mathrm{id}_{A}=0$ pour un certain $n \in \mathbb{N}-\{0\}$. Alors, $\mathcal{T}_{\text {tor }}$ est une sous-catégorie triangulée de $\mathcal{T}$ et le foncteur évident $\mathcal{T} \longrightarrow \mathcal{T} \otimes \mathbb{Q}$ identifie $\mathcal{T} \otimes \mathbb{Q}$ avec le quotient de Verdier $\mathcal{T} / \mathcal{T}_{\text {tor. }}$ En particulier, $\mathcal{T} \otimes \mathbb{Q}$ est naturellement une catégorie triangulée.

Demonstration La catégorie $\mathcal{T}_{\text {tor }}$ est clairement stable par les foncteurs de suspension et de désuspension. Supposons donné un triangle distingué dans $\mathcal{T}$ :

$$
A \longrightarrow B \longrightarrow C \longrightarrow A[1] \text {. }
$$

Si $m \cdot \operatorname{id}_{A}=0$ et $n \cdot \operatorname{id}_{B}=0$, la multiplication par $m n$ dans le triangle distingué ci-dessus est nulle sur les deux premiers sommets. Il vient que $(m n) \cdot \mathrm{id}_{C}$ se factorise par $C \longrightarrow A[1]$ et $B \longrightarrow C$. Il en découle aussitôt que son carré est nul. Autrement dit, on a $(m n)^{2} \cdot \operatorname{id}_{C}=0$ et $C \in \mathcal{T}_{\text {tor }}$. Ceci montre que $\mathcal{T}_{\text {tor }}$ est une sous-catégorie triangulée de $\mathcal{T}$.

Par ailleurs, pour tout $M \in \mathcal{T}$ et $n \in \mathbb{N}-\{0\}$, on peut former un triangle distingué

$$
M \stackrel{n \cdot \mathrm{id}}{\longrightarrow} M \longrightarrow Q_{n}(M) \longrightarrow M[1] .
$$

Par construction, le morphisme $n \cdot \operatorname{id}_{Q_{n}(M)}$ composé avec $Q_{n}(M) \longrightarrow M[1]$ est nul. Il se factorise donc par $M \longrightarrow Q_{n}(M)$. Or, le $n$-ième multiple de cette flèche est nulle. On en déduit que $n^{2}$. $\operatorname{id}_{Q_{n}(M)}=0$ et donc que $Q_{n}(M)$ est dans $\mathcal{T}_{\text {tor }}$. Il s'ensuit aussitôt que $\mathcal{V}=\mathcal{T} / \mathcal{T}_{\text {tor }}$ est $\mathbb{Q}$-linéaire. Le foncteur évident $\mathcal{T} \longrightarrow \mathcal{V}$ induit alors un foncteur $\mathcal{T} \otimes \mathbb{Q} \longrightarrow \mathcal{V}$ qui est l'identité sur les objets. Il reste à montrer que ce foncteur est pleinement fidèle.

D'après la construction du quotient de Verdier, une flèche $\alpha: A \longrightarrow B$ dans $\mathcal{V}$ est une classe d'équivalence de diagrammes de la forme

$$
A \stackrel{u}{\longleftarrow} A^{\prime} \stackrel{a}{\longrightarrow} B
$$

avec Cône $(u) \in \mathcal{T}_{\text {tor }}$. Si $n \cdot \operatorname{id}_{\operatorname{Cone}(u)}=0$, le morphisme $n \cdot \operatorname{id}_{A}$ composé avec $A \longrightarrow$ Cône $(u)$ est nul. Il se factorise donc par $u$. Autrement dit, il existe une flèche $v: A \longrightarrow A^{\prime}$ telle que $u \circ v=n \cdot \operatorname{id}_{A}$. On peut donc supposer que $A^{\prime}=A$ et $u=n \cdot \mathrm{id}_{A}$. Dans ce cas, $n \cdot \alpha=a$. Ceci montre que le foncteur $\mathcal{T} \otimes \mathbb{Q} \longrightarrow \mathcal{V}$ est plein.

D'autre part, pour montrer que $\operatorname{hom}_{\mathcal{T}}(A, B) \otimes_{\mathbb{Z}} \mathbb{Q} \longrightarrow \operatorname{hom}_{\mathcal{V}}(A, B)$ est injectif il suffit de voir que le noyau de $\operatorname{hom}_{\mathcal{T}}(A, B) \longrightarrow \operatorname{hom}_{\mathcal{V}}(A, B)$ est un sous-groupe de torsion. Soit $b \in \operatorname{hom}_{\mathcal{T}}(A, B)$ une flèche et supposons qu'elle devient nulle dans $\mathcal{V}$. D'après la construction du quotient de Verdier, il existe une flèche $u: A^{\prime} \longrightarrow A$ telle que Cône $(u) \in \mathcal{T}_{\text {tor }}$ et $b \circ u=0$. Comme ci-dessus, on peut trouver une flèche $v: A \longrightarrow A^{\prime}$ telle que $u \circ v=n \cdot \operatorname{id}_{A}$ pour un certain $n \in \mathbb{N}-\{0\}$. Il en découle alors que $n \cdot b=0$. La proposition est démontrée.

Par ailleurs, on a le fait suivant.

Proposition 9.2 - Soient $S$ un schéma et $\Lambda$ un anneau de coefficients. Le foncteur $-\otimes \mathbb{Q}$ : $\mathbf{D A}_{\mathrm{ct}}^{\mathrm{et}}(S, \Lambda) \longrightarrow \mathbf{D A}_{\mathrm{ct}}^{\mathrm{et}}(S, \Lambda \otimes \mathbb{Q})$ induit un foncteur triangulé

$$
\mathbf{D A}_{\mathrm{ct}}^{\mathrm{et}}(S, \Lambda) \otimes \mathbb{Q} \longrightarrow \mathbf{D} \mathbf{A}_{\mathrm{ct}}^{\mathrm{et}}(S, \Lambda \otimes \mathbb{Q})
$$


Si de plus l'Hypothèse 3.17 et satisfaite, le foncteur (86) induit une équivalence de catégories entre $\mathbf{D} \mathbf{A}_{\mathrm{ct}}^{\mathrm{et}}(S, \Lambda \otimes \mathbb{Q})$ et l'enveloppe pseudo-abélienne de $\mathbf{D A}_{\mathrm{ct}}^{\mathrm{et}}(S, \Lambda) \otimes \mathbb{Q}$.

Demonstration Remarquons que $-\otimes \mathbb{Q}$ préserve les objets constructibles. La première partie de l'énoncé découle formellement du fait que $\mathbf{D A}_{\mathrm{ct}}^{\text {ét }}(S, \Lambda \otimes \mathbb{Q})$ est $\mathbb{Q}$-linéaire. On passe à la seconde partie. Notons $a^{*}: \mathbf{D A}^{\text {ét }}(S, \Lambda) \longrightarrow \mathbf{D A}^{\text {ét }}(S, \Lambda \otimes \mathbb{Q})$ le foncteur $-\otimes \mathbb{Q}$ et $a_{*}$ son adjoint à droite. Pour $A, B \in \mathbf{D A}^{\text {ét }}(S, \Lambda)$, on a

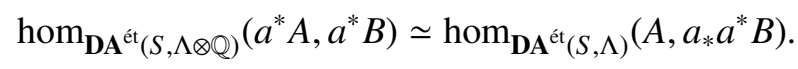

D'après le Lemme A.4, on voit que $a_{*} a^{*} B=B \otimes \mathbb{Q}$ vu comme un objet de $\mathbf{D} A^{\text {ét }}(S, \Lambda)$. C'est donc la colimite filtrante du système $(B)_{n \in \mathbb{N}^{\times}}$où les morphismes de transition associés à $m$ divisant $n$ sont donnés par $\frac{n}{m} \cdot \operatorname{id}_{B}$. Or, si $A$ est constructible il est compact par la Proposition 8.3 et on obtient des identifications canoniques

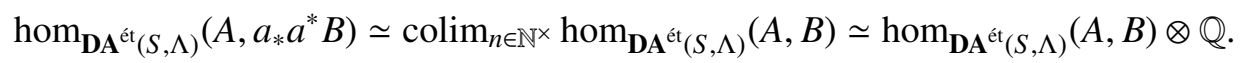

Ceci montre que le foncteur $\mathbf{D} A_{\mathrm{ct}}^{\mathrm{et}}(S, \Lambda) \otimes \mathbb{Q} \longrightarrow \mathbf{D A}_{\mathrm{ct}}^{\text {et }}(S, \Lambda \otimes \mathbb{Q})$ est pleinement fidèle. De plus, c'est un foncteur triangulé par la Proposition 9.1. Par ailleurs, l'image de ce foncteur contient des générateurs de $\mathbf{D} \mathbf{A}_{\mathrm{ct}}^{\mathrm{et}}(S, \Lambda \otimes \mathbb{Q}$ ) comme catégorie triangulée stable par facteurs directs (cf. la Définition 8.1). Il en découle que tout objet de $\mathbf{D} A_{\mathrm{ct}}^{\text {ét }}(S, \Lambda \otimes \mathbb{Q})$ est un facteur direct d'un objet de l'image de $\mathbf{D} A_{\mathrm{ct}}^{\text {ét }}(S, \Lambda) \otimes \mathbb{Q}$. Ceci termine la preuve de la proposition puisque $\mathbf{D} \mathbf{A}_{\mathrm{ct}}^{\mathrm{et}}(S, \Lambda \otimes \mathbb{Q})$ est pseudo-abélienne.

Definition 9.3 - Soient $S$ un schéma, $\Lambda$ un anneau et $J \subset \Lambda$ un idéal. On note $\hat{\mathbf{D}}_{\mathrm{ct}}^{\text {ét }}\left(S, \Lambda_{J}\right)$ la sous-catégorie pleine de $\hat{\mathbf{D}}^{\mathrm{e} t}\left(S, \Lambda_{J}\right)$ dont les objets sont les complexes de préfaisceaux de $\Lambda / J^{*}$ modules $K$ tels que pour tout $s \in \mathbb{N}$, le complexe $s^{*} K$ est constructible au sens suivant. Les fais-

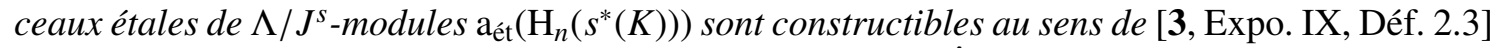
et ils sont nuls pour $|n|$ suffisamment grand. On définit alors $\hat{\mathbf{D}}_{\mathrm{ct}}^{\mathrm{et}}\left(S, \Lambda_{J} \otimes \mathbb{Q}\right)$ comme étant l'enveloppe pseudo-abélienne de $\hat{\mathbf{D}}_{\mathrm{ct}}^{\mathrm{et}}\left(S, \Lambda_{J}\right) \otimes \mathbb{Q}$. Lorsque $\Lambda \subset \mathbb{Z}_{(\ell)}$ et $J=(\ell)$, pour un nombre premier $\ell$, on note simplement $\hat{\mathbf{D}}_{\mathrm{ct}}^{\mathrm{et}}\left(S, \mathbb{Q}_{\ell}\right)$ la catégorie ainsi définie.

Remarque 9.4 - Remarquons que $\hat{\mathbf{D}}_{\mathrm{ct}}^{\text {ét }}\left(S, \Lambda_{J} \otimes \mathbb{Q}\right)$ est naturellement une catégorie triangulée. En effet $\hat{\mathbf{D}}_{\mathrm{ct}}^{\text {et }}\left(S, \Lambda_{J}\right) \otimes \mathbb{Q}$, est une catégorie triangulée d'après la Proposition 9.1. De plus, l'enveloppe pseudo-abélienne d'une catégorie triangulée est encore une catégorie triangulée d'après [9].

Proposition 9.5 - Soient $S$ un schéma, $\Lambda$ un anneau et $J \subset \Lambda$ un idéal. On suppose que l'Hypothèse 5.1 est satisfaite. Alors, la réalisation étale $\Re_{S}^{\mathrm{e} t}: \mathbf{D A}^{\mathrm{ét}}(S, \Lambda) \longrightarrow \hat{\mathbf{D}}^{\mathrm{ét}}\left(S, \Lambda_{J}\right)$ envoie la sous-catégorie $\mathbf{D A}_{\mathrm{ct}}^{\mathrm{et}}(S, \Lambda)$ dans $\hat{\mathbf{D}}_{\mathrm{ct}}^{\text {et }}\left(S, \Lambda_{J}\right)$. Elle induit donc un foncteur

$$
\Re_{S}^{\text {ét }}: \mathbf{D} A_{\mathrm{ct}}^{\text {ét }}(S, \Lambda) \longrightarrow \hat{\mathbf{D}}_{\mathrm{ct}}^{\text {ét }}\left(S, \Lambda_{J}\right) .
$$

Demonstration D'après [4, Lem. 2.2.23], $\mathbf{D A}_{\mathrm{ct}}^{\text {ét }}(S, \Lambda)$ est la plus petite sous-catégorie triangulée de $\mathbf{D A}{ }^{\text {ét }}(S, \Lambda)$ stable par facteurs directs et contenant les motifs de la forme $f_{*} \Lambda_{X}(n)$ avec $n \in$ $\mathbb{Z}$ et $f: X \longrightarrow S$ un morphisme projectif. Or, il est immédiat que $\mathfrak{R}_{X}^{\text {ét }}\left(\Lambda_{X}(n)\right) \simeq\left(\Lambda / J^{*}\right)(n)$ est dans $\hat{\mathbf{D}}_{\mathrm{ct}}^{\text {ét }}\left(X, \Lambda_{J}\right)$. D'autre part, l'opération $f_{*}$ commute à la réalisation étale d'après le Théorème 6.9. Il est donc suffisant de montrer que l'opération $f_{*}$ préserve les sous-catégories $\hat{\mathbf{D}}_{\mathrm{ct}}^{\text {et }}\left(-, \Lambda_{J}\right)$. Ceci revient à montrer que $f_{*}$ préserve les complexes constructibles de faisceaux étales de $\Lambda / J^{s}$ modules. Puisque $f$ est projectif, ceci découle facilement de [3, Expo. XIV, Th. 1.1].

Definition 9.6 - Soient $S$ un schéma, $\Lambda$ un anneau et $J \subset \Lambda$ un idéal. On suppose que $l$ 'Hypothèse 6.5 est satisfaite. Le foncteur (87) induit un foncteur triangulé entre $\mathbf{D} \mathbf{A}_{\mathrm{ct}}^{\mathrm{et}}(S, \Lambda) \otimes \mathbb{Q}$ et $\hat{\mathbf{D}}_{\mathrm{ct}}^{\mathrm{et}}\left(S, \Lambda_{J}\right) \otimes \mathbb{Q}$. En passant aux enveloppes pseudo-abéliennes et en utilisant la Proposition 9.2, on obtient un foncteur triangulé, monoïdal symétrique et unitaire

$$
\Re_{S}^{\text {ét }}: \mathbf{D A}_{\mathrm{ct}}^{\text {ét }}(S, \Lambda \otimes \mathbb{Q}) \longrightarrow \hat{\mathbf{D}}_{\mathrm{ct}}^{\text {et }}\left(S, \Lambda_{J} \otimes \mathbb{Q}\right) \text {. }
$$

Ce foncteur est la réalisation étale à coefficients uniquement divisibles. 
Theoreme 9.7 - Soient $S$ un schéma de base excellent, $\Lambda$ un anneau de coefficients et $J \subset \Lambda$ un idéal tel que $\Lambda / J$ est de torsion (en tant que $\mathbb{Z}$-module). On suppose que l'Hypothèse 7.3 est satisfaite. Alors, les foncteurs de réalisations étales $\Re^{\mathrm{ét}}: \mathbf{D A}_{\mathrm{ct}}^{\mathrm{et}}(-, \Lambda \otimes \mathbb{Q}) \longrightarrow \hat{\mathbf{D}}_{\mathrm{ct}}^{\mathrm{et}}\left(-, \Lambda_{J} \otimes \mathbb{Q}\right)$ commutent aux six opérations de Grothendieck pour les $S$-schémas quasi-projectifs. Plus précisément, les propriétés $(\boldsymbol{A})$ et $(\boldsymbol{B}) d u$ Théorème 6.6 sont satisfaites pour ces foncteurs.

Demonstration On a des foncteurs triangulés $\Re^{\text {ét }} \otimes \mathbb{Q}: \mathbf{D A}^{\text {ét }}(-, \Lambda) \otimes \mathbb{Q} \longrightarrow \hat{\mathbf{D}}^{\text {ét }}\left(-, \Lambda_{J}\right) \otimes \mathbb{Q}$ qui commutent aux six opérations de Grothendieck d'après le Théorème 6.9. Or, les six opérations de Grothendieck préservent les sous-catégories des objets constructibles d'après les Théorèmes 8.10 et 8.12. Il suffit alors de passer aux enveloppes pseudo-abéliennes et d'utiliser la Proposition 9.2 pour conclure.

\section{Motifs proches et cycles évanescents}

Dans ce paragraphe nous nous proposons d'étudier le lien entre le formalisme des motifs proches de [5, Chap. 3], et le formalisme des cycles évanescents de [18, Expo. I] et [12, Expo. XIII] à l'aide de la réalisation étale construite dans les Sections 5 et 9 . Nous commençons d'abord par des rappels et des compléments sur le formalisme des motifs proches.

Soit $S$ un schéma de base. (Plus tard, $S$ sera le spectre d'un anneau de valuation discrète.) On note $i: S \hookrightarrow \mathbb{A}_{S}^{1}$ la section nulle et $j: \mathbb{G m}_{S} \hookrightarrow \mathbb{A}_{S}^{1}$ l'immersion ouverte complémentaire.

Rappellons d'abord la construction du foncteur «partie unipotente du motif proche ». Elle repose sur un $S$-schéma cosimplicial $\mathscr{A}_{S}$. Il est donné par $\mathscr{A}_{S}(\underline{\mathbf{n}})=(\mathbb{G} \mathrm{m})_{S}^{n+1}$ pour $\underline{\mathbf{n}} \in \Delta$, les faces sont induites par le morphisme diagonal $\Delta: \mathbb{G}_{S} \longrightarrow(\mathbb{G})_{S}^{2}$ et la section unité $1: S \hookrightarrow \mathbb{G}_{S}$, et des dégénérescences sont les projections partielles. Pour la description exacte du diagramme de $S$-schémas $\left(\mathscr{A}_{S}, \Delta\right)$, nous renvoyons le lecteur à [5, Déf. 3.4.4]. Nous disposons d'un morphisme canonique de $\Delta$-diagrammes de schémas

$$
\theta^{\mathscr{A}}:\left(\mathscr{A}_{S}, \Delta\right) \longrightarrow\left(\mathbb{G}_{S}, \Delta\right) \text {. }
$$

Il est donné en $\underline{\mathbf{n}} \in \Delta$ par la projection sur le premier facteur de $(\mathbb{G m})_{S}^{n+1}$. Étant donné un morphisme de $S$-schémas quasi-projectifs $f: X \longrightarrow \mathbb{A}_{S}^{1}$, on forme le diagramme commutatif à carrés cartésiens :

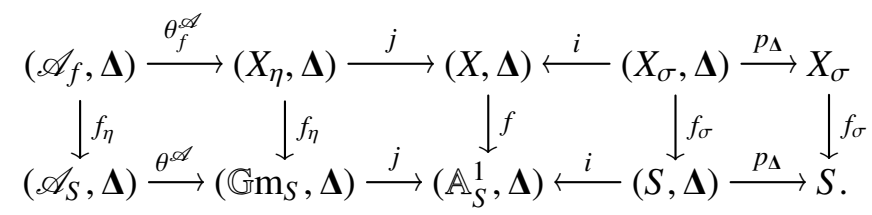

On définit alors le foncteur $\Upsilon_{f}: \mathbf{D} A^{\text {ét }}\left(X_{\eta}, \Lambda\right) \longrightarrow \mathbf{D A}^{\text {ét }}\left(X_{\sigma}, \Lambda\right)$ par

$$
\Upsilon_{f}=\left(p_{\Delta}\right)_{\sharp} i^{*} j_{*}\left(\theta_{f}^{\mathscr{A}}\right)_{*}\left(\theta_{f}^{\mathscr{A}}\right)^{*} p_{\Delta}^{*} .
$$

On obtient ainsi un système de spécialisation pseudo-monoïdal $\Upsilon$ au-dessus de $\left(\mathbb{A}_{S}^{1}, j, i\right)$ au sens de [5, Déf. 3.1.1 et Déf. 3.1.12]. Les foncteurs $\Upsilon_{f}$ sont appelés les foncteurs « partie unipotente du motif proche ». Par construction, on a un morphisme de systèmes de spécialisation pseudomonoïdaux $\chi \longrightarrow \Upsilon$ où $\chi$ est le système de spécialisation canonique donné par $\chi_{f}=i^{*} j_{*}$. Le $S$-schéma cosimplicial $\mathscr{A}_{S}$ est spécialement désigné pour que le résultat suivant soit vrai.

Proposition 10.1 - Appelons $p: \mathbb{A}_{S}^{1} \longrightarrow S$ et $q=p \circ j: \mathbb{G}_{S} \longrightarrow S$ les projections canoniques. Alors, la composition de

$$
\mathrm{id} \simeq i^{*} p^{*} \stackrel{\eta}{\longrightarrow} i^{*} j_{*} j^{*} p^{*} \simeq \chi_{\mathrm{id}} \circ q^{*} \longrightarrow \Upsilon_{\mathrm{id}} \circ q^{*}
$$

est un 2-isomorphisme.

Demonstration Il s'agit d'un cas particulier de [5, Prop. 3.4.9].

Le résultat suivant est désormais possible grâce à la pureté absolue pour les motifs étales. 
Theoreme 10.2 - Soient $S$ un schéma de base et $\Lambda$ un anneau de coefficients. On suppose que l'Hypothèse 7.3 est satisfaite. On se donne un morphisme de $S$-schémas quasi-projectifs $f$ : $X \longrightarrow \mathbb{A}_{S}^{1}$ avec $X$ régulier. On suppose que $\left(X_{\sigma}\right)_{\text {red }}$ est régulier et que les longueurs des anneaux locaux de $X_{\sigma}$ en ses points génériques sont inversibles dans $\Lambda$. Alors, la composition de

$$
\Lambda_{X_{\sigma}} \longrightarrow \chi_{f} \Lambda_{X_{\eta}} \longrightarrow \Upsilon_{f} \Lambda_{X_{\eta}}
$$

est un isomorphisme dans $\mathbf{D} \mathbf{A}^{\mathrm{ét}}\left(X_{\sigma}, \Lambda\right)$.

Demonstration Quitte à remplacer $S$ par $X$, on peut supposer que le schéma de base $S$ est régulier. Soit $t: X \longrightarrow W$ une immersion fermée dans un $\mathbb{A}_{S}^{1}$-schéma lisse $g: W \longrightarrow \mathbb{A}_{S}^{1}$. Le morphisme de changement de base $g_{\sigma}^{*} \Upsilon_{\text {id }} \longrightarrow \Upsilon_{g} g_{\eta}^{*}$ est inversible puisque $g$ est lisse et que $\Upsilon$ est un système de spécialisation (cf. [5, Déf. 3.1.1]). D'après la Proposition 10.1, le théorème est donc vrai pour $W$. Autrement dit, la composition de

$$
\Lambda_{W_{\sigma}} \longrightarrow \chi_{g} \Lambda_{W_{\eta}} \longrightarrow \Upsilon_{g} \Lambda_{W_{\eta}}
$$

est un isomorphisme. Pour conclure, il suffira alors de montrer que le morphisme naturel

$$
t_{\sigma}^{*} \Upsilon_{g} \Lambda_{W_{\eta}} \longrightarrow \Upsilon_{f} t_{\eta}^{*} \Lambda_{W_{\eta}}
$$

est inversible. En revenant à la définition du morphisme de changement de base $t_{\sigma}^{*} \Upsilon_{g} \longrightarrow \Upsilon_{f} t_{\eta}^{*}$, on voit qu'il suffit de montrer que le morphisme naturel

$$
t^{*} j_{*}\left(\theta_{g}^{\mathscr{A}}\right)_{*} \Lambda \longrightarrow j_{*}\left(\theta_{f}^{\mathscr{A}}\right)_{*} t_{\eta}^{*} \Lambda
$$

est inversible. Il s'agit là d'un morphisme de $\mathbf{D} \mathbf{A}^{\text {ét }}((X, \boldsymbol{\Delta}), \Lambda)$ et pour montrer qu'il est inversible il suffit de le faire après application des foncteurs $\underline{\mathbf{n}}^{*}: \mathbf{D A}^{\text {ét }}((X, \Delta), \Lambda) \longrightarrow \mathbf{D A}^{\text {ét }}(X, \Lambda)$ pour $n \in \mathbb{N}$. Vu que $\theta_{g}^{\mathscr{A}}(\underline{\mathbf{n}}): W_{\eta} \times_{S}(\mathbb{G m})_{S}^{n} \longrightarrow W_{\eta}$ est la projection sur le premier facteur, on a une identification canonique

$$
\left(\theta_{g}^{\mathscr{A}}(\underline{\mathbf{n}})\right)_{*} \Lambda \simeq(\Lambda \oplus \Lambda(-1)[-1])^{\otimes n}
$$

et le morphisme naturel $t_{\eta}^{*}\left(\theta_{g}^{\mathscr{A}}\right)_{*} \Lambda \longrightarrow\left(\theta_{f}^{\mathscr{A}}\right)_{*} t_{\eta}^{*} \Lambda$ est inversible. Les propriétés basiques des dérivateurs algébriques et notamment l'axiome DerAlg 3d de [4, page 441], nous ramène alors à vérifier que le morphisme $t^{*} j_{*} \Lambda(r) \longrightarrow j_{*} t_{\eta}^{*} \Lambda(r)$ est inversible pour tout $r \in \mathbb{Z}$. Ceci découle de la Proposition 10.3 ci-dessous et du 2-triangle distingué de localité de [4, Prop. 1.4.9].

Proposition 10.3 - Soient $S$ un schéma de base et $\Lambda$ un anneau de coefficients satisfaisant à l'Hypothèse 7.3. Supposons donné un carré cartésien de S-schémas quasi-projectifs

$$
\begin{array}{ccc}
Z^{\prime} \stackrel{g^{\prime}}{\longrightarrow} & Z \\
\downarrow_{t^{\prime}} & & \rfloor t \\
X^{\prime} & g & \stackrel{X}{\longrightarrow}
\end{array}
$$

tel que $X, X^{\prime}, Z$ et $\left(Z^{\prime}\right)_{\text {red }}$ sont réguliers, $t$ et $t^{\prime}$ sont des immersions fermées partout de codimension 1 , et les longueurs des anneaux locaux aux points génériques de $Z^{\prime}$ sont égales à $r \in \mathbb{N}-\{0\}$. Supposons aussi que les idéaux des immersions fermées $t: Z \hookrightarrow X$ et $t_{\text {red }}^{\prime}:\left(Z^{\prime}\right)_{\text {red }} \longrightarrow X^{\prime}$ sont libres engendrés respectivement par $f \in \Gamma(X, \mathcal{O})$ et $f^{\prime} \in \Gamma\left(X^{\prime}, \mathcal{O}\right)$. Alors, la composition de

$$
\Lambda_{Z^{\prime}}(-1)[-2] \simeq g^{\prime *} t^{!} \Lambda_{X} \longrightarrow t^{\prime} g^{*} \Lambda_{X} \simeq \Lambda_{Z^{\prime}}(-1)[-2]
$$

est la multiplication par $r$ dans $\mathbf{D A}^{\mathrm{et}}\left(Z^{\prime}, \Lambda\right)$. (Ci-dessus, les isomorphismes $t^{!} \Lambda_{X} \simeq \Lambda_{Z}(-1)[-2]$ et $t^{\prime} \Lambda_{X^{\prime}} \simeq \Lambda_{Z^{\prime}}(-1)[-2]$ sont ceux $d u$ Corollaire 7.5.)

Demonstration Considérons le morphisme $\alpha: t_{*} \Lambda_{Z} \longrightarrow \Lambda_{X}(1)$ [2] donné par la composition de

$$
t_{*} \Lambda_{Z} \simeq t_{*} t^{\prime} \Lambda_{X}(1)[2] \longrightarrow \Lambda_{X}(1)[2]
$$

(l'isomorphisme $\Lambda_{Z} \simeq t^{!} \Lambda_{X}(1)$ [2] étant celui du Corollaire 7.5) ainsi que le morphisme analogue $\alpha^{\prime}: t_{*}^{\prime} \Lambda_{Z^{\prime}} \longrightarrow \Lambda_{X^{\prime}}(1)[2]$. Il suffit de prouver que la composition de

$$
t_{*}^{\prime} \Lambda_{Z^{\prime}} \simeq t_{*}^{\prime} g^{\prime *} \Lambda_{Z} \simeq g^{*} t_{*} \Lambda_{Z} \stackrel{\alpha}{\longrightarrow} g^{*} \Lambda_{X}(1)[2] \simeq \Lambda_{X^{\prime}}(1)[2]
$$


est égale à $r \cdot \alpha^{\prime}$.

Le Lemme 10.4 ci-dessous fournit une description explicite du morphisme $\alpha$. On en déduit aussitôt que la composition de (92) s'identifie canoniquement à la $T$-suspension infinie du morphisme de préfaisceaux

$$
\frac{X^{\prime} \otimes \Lambda}{\left(X^{\prime}-Z^{\prime}\right) \otimes \Lambda} \stackrel{f \circ g}{\longrightarrow} \frac{\mathbb{A}_{X^{\prime}}^{1} \otimes \Lambda}{\left(\mathbb{A}_{X^{\prime}}^{1}-0_{X^{\prime}}\right) \otimes \Lambda} .
$$

Par ailleurs, on a une relation $f \circ g=u \cdot f^{\prime r}$ avec $u \in \Gamma\left(X^{\prime}, \mathcal{O}^{\times}\right)$. En utilisant la Proposition 2.6, en fait sa version homologique, on obtient que la $T$-suspension infinie de (93) est égale dans $\mathbf{D A}^{\text {ét }}\left(X^{\prime}, \Lambda\right)$ à la $T$-suspension infinie de la somme (cf. la Remarque 10.5 ci-dessous)

$$
r \cdot\left[\frac{X^{\prime} \otimes \Lambda}{\left(X^{\prime}-Z^{\prime}\right) \otimes \Lambda} \stackrel{f^{\prime}}{\longrightarrow} \frac{\mathbb{A}_{X^{\prime}}^{1} \otimes \Lambda}{\left(\mathbb{A}_{X^{\prime}}^{1}-0_{X^{\prime}}\right) \otimes \Lambda}\right]+\left[\frac{X^{\prime} \otimes \Lambda}{\left(X^{\prime}-Z^{\prime}\right) \otimes \Lambda} \stackrel{u}{\longrightarrow} \frac{\mathbb{A}_{X^{\prime}}^{1} \otimes \Lambda}{\left(\mathbb{A}_{X^{\prime}}^{1}-0_{X^{\prime}}\right) \otimes \Lambda}\right] .
$$

Or, le morphisme $u: X^{\prime} \longrightarrow \mathbb{A}_{X^{\prime}}^{1}$ se factorise par $\mathbb{A}_{X^{\prime}}^{1}-0_{X^{\prime}}$. Il en découle que le second membre de (94) est la flèche nulle. Une seconde application du Lemme 10.4 ci-dessous permet alors de conclure.

Lemme 10.4 - Soient $S$ un schéma régulier et $\Lambda$ un anneau de coefficients satisfaisant à $l$ 'Hypothèse 7.3. Soit $s: T \hookrightarrow S$ une immersion fermée partout de codimension 1 avec $T$ un schéma régulier. On suppose que l'idéal de l'immersion s est libre engendré par $f \in \Gamma(S, \mathcal{O})$. Alors, la composition de

$$
s_{*} \Lambda_{T}(0) \simeq s_{*} s^{!} \Lambda(1)[2] \longrightarrow \Lambda_{S}(1)[2],
$$

avec $\Lambda_{T} \simeq s^{!} \Lambda(1)$ [2] l'isomorphisme de pureté du Corollaire 7.5, s'identifie canoniquement dans $\mathbf{D A}^{\mathrm{e}}{ }^{\mathrm{t}}(S, \Lambda)$ à la $T$-suspension infinie du morphisme

$$
\frac{S \otimes \Lambda}{(S-T) \otimes \Lambda} \stackrel{f}{\longrightarrow} \frac{\mathbb{A}_{S}^{1} \otimes \Lambda}{\left(\mathbb{A}_{S}^{1}-0_{S}\right) \otimes \Lambda}
$$

induit par la section $f: S \longrightarrow \mathbb{A}_{S}^{1}$.

Demonstration D'après le Théorème 7.4 et la construction de l'isomorphisme de pureté du Corollaire 7.5, il suffit de traiter la cas où $S=\mathbb{A}_{T}^{1}$ et $s: T \hookrightarrow \mathbb{A}_{T}^{1}$ la section nulle. (Bien entendu, on prendra pour $f$ l'indéterminée $\pi$ telle que $\mathbb{A}_{T}^{1}=\operatorname{Spec}\left(\mathcal{O}_{T}[\pi]\right)$.)

Notons $p: \mathbb{A}_{T}^{1} \longrightarrow T$ la projection canonique. Alors $\Lambda(1)[2]=p_{\sharp} S_{*} \Lambda(0)$ est canoniquement isomorphe à la $T$-suspension infinie de $\mathbb{A}_{T}^{1} \otimes \Lambda /\left(\mathbb{A}_{T}^{1}-0_{T}\right) \otimes \Lambda$. (Ceci résulte du triangle de localité de $\left[4\right.$, Lem. 1.4.6].) On cherche à déterminer le morphisme de counité $s_{*} \Lambda_{T} \longrightarrow p^{*} p_{\sharp} S_{*} \Lambda_{T}$ modulo cette identification. En retournant à la construction des opérations $p_{\sharp}$ et $p^{*}$, on voit qu'il s'agit de la $T$-suspension infinie du morphisme de préfaisceaux

$$
\frac{\mathbb{A}_{T}^{1} \otimes \Lambda}{\left(\mathbb{A}_{T}^{1}-0_{T}\right) \otimes \Lambda} \stackrel{\Delta}{\longrightarrow} \frac{\left(\mathbb{A}_{T}^{1} \times_{T} \mathbb{A}_{T}^{1}\right) \otimes \Lambda}{\left(\left(\mathbb{A}_{T}^{1}-0_{T}\right) \times_{T} \mathbb{A}_{T}^{1}\right) \otimes \Lambda}
$$

induit par le le morphisme diagonal $\Delta: \mathbb{A}_{T}^{1} \longrightarrow \mathbb{A}_{T}^{1} \times_{T} \mathbb{A}_{T}^{1}$. Le lemme est démontré.

Remarque 10.5 - Le lecteur attentif a peut-être remarqué un usage abusif de la Proposition 2.6 dans la preuve de la Proposition 10.3 ci-dessus. En effet, le cas où 2 n'est pas inversible dans $\Lambda$ n'est pas couvert par la Proposition 2.6 (à moins que $S$ ne soit un $\mathbb{F}_{2}$-schéma). Heureusement, pour les catégories $\mathbf{D} \mathbf{A}^{\text {ét }}(-, \Lambda)$ on peut s'en sortir grâce au Théorème B.1. En effet, on a besoin de savoir que la $T$-suspension infinie du morphisme

$$
\frac{\left(\mathbb{A}_{S}^{1} \times \mathbb{A}_{S}^{1}\right) \otimes \Lambda}{\left(\mathbb{G m}_{S} \times S \mathrm{Gm}_{S}\right) \otimes \Lambda} \stackrel{m-p_{1}-p_{2}}{\longrightarrow} \frac{\mathbb{A}_{S}^{1} \otimes \Lambda}{\mathbb{G m}_{S} \otimes \Lambda}
$$

est nulle dans $\mathbf{D A}{ }^{\text {ét }}(S, \Lambda)$. (Bien entendu, $m$ est la multiplication et $p_{i}$ est la projection sur le $i$-ième facteur.) Il suffit de faire cela pour $S$ le spectre d'un sous-anneau de $\mathbb{Q}$ et on peut donc supposer 
que $S$ est régulier. Grâce au Théorème B.1, il est suffisant de montrer que

$$
\frac{\mathbb{Z}_{t r}\left(\mathbb{A}_{S}^{1} \times_{S} \mathbb{A}_{S}^{1}\right)}{\mathbb{Z}_{t r}\left(\mathbb{G m}_{S} \times_{S} \mathbb{G}_{S}\right)} \stackrel{m-p_{1}-p_{2}}{\longrightarrow} \frac{\mathbb{Z}_{t r}\left(\mathbb{A}_{S}^{1}\right)}{\mathbb{Z}_{t r}\left(\mathbb{G m}_{S}\right)}
$$

est nul dans $\mathbf{D M}^{\text {eff }}(S, \mathbb{Z})$. Il revient au même de montrer que le morphisme

$$
m-p_{1}-p_{2}: \mathbb{Z}_{t r}\left(\mathbb{G m}_{S}^{2}, 1 \times 1\right) \longrightarrow \mathbb{Z}_{t r}\left(\mathbb{G} \mathrm{m}_{S}, 1\right)
$$

est nul. Cette propriété découle aussitôt du fait que le morphisme $\mathbb{Z}_{t r}\left(G^{\mathrm{G}} \mathrm{m}_{S}, 1\right) \longrightarrow \mathcal{O}^{\times}$est inversible dans $\mathbf{D M}^{\text {eff }}(S, \mathbb{Z})$. Une preuve de cela s'obtient en calquant la preuve de [31, Th. 4.1] (ce qui ne présente pas de difficulté en supposant $S$ régulier).

Passons maintenant à la construction des foncteurs «partie modérée du motif proche ». Nous disposons d'un diagramme de $S$-schémas $\left(\mathscr{E}_{S}, \mathbb{N}^{\times}\right)$défini de la manière suivante. La catégorie $\mathbb{N}^{\times}$est l'ensemble $\mathbb{N}-\{0\}$ ordonné par l'opposé de la relation de divisibilité. Pour $n \in \mathbb{N}^{\times}$on a $\mathscr{E}_{S}(n)=\mathbb{G}_{S}$ et si $m \mid n$, le morphisme $\mathscr{E}_{S}(n) \longrightarrow \mathscr{E}_{S}(m)$ est l'élévation à la puissance $n m^{-1}$ sur $\mathbb{G}_{S}$. Dans $[\mathbf{5}, \S 3.5 .1]$ nous avions introduit un diagramme de schémas $\left(\mathscr{R}_{S}, \Delta \times \mathbb{N}^{\times}\right)$. Il est construit à partir de $\left(\mathscr{E}_{S}, \mathbb{N}^{\times}\right)$de la même manière que $\left(\mathscr{A}_{S}, \Delta\right)$ est construit à partir de $\mathbb{G}_{S}$. En particulier, on a un morphisme de diagrammes de $S$-schémas $\left(\mathscr{R}_{S}, \Delta \times \mathbb{N}^{\times}\right) \longrightarrow\left(\mathscr{E}_{S}, \mathbb{N}^{\times}\right)$. En composant avec le morphisme $\left(\mathscr{E}_{S}, \mathbb{N}^{\times}\right) \longrightarrow \mathbb{G}_{S}$, donné en $n \in \mathbb{N}^{\times}$par l'élévation à la puissance $n$ sur $\mathbb{G m}_{S}$, on obtient un morphisme de $\left(\Delta \times \mathbb{N}^{\times}\right)$-diagrammes de $S$-schémas

$$
\theta^{\mathscr{R}}:\left(\mathscr{R}_{S}, \Delta \times \mathbb{N}^{\times}\right) \longrightarrow\left(\mathbb{G m}_{S}, \Delta \times \mathbb{N}^{\times}\right) .
$$

Notons $\mathbb{N}^{\prime \times}$ le sous-ensemble ordonné de $\mathbb{N}^{\times}$formé des entiers inversibles sur $S$. Soient $\mathscr{E}_{S}^{\prime}$ et $\mathscr{R}_{S}^{\prime}$ les restrictions de $\mathscr{E}_{S}$ et $\mathscr{R}_{S}$ à $\mathbb{N}^{\prime \times}$ et $\Delta \times \mathbb{N}^{\prime \times}$ respectivement. Nous déduisons de (95) un morphisme de $\left(\Delta \times \mathbb{N}^{\prime \times}\right)$-schémas

$$
\theta^{\mathscr{R}^{\prime}}:\left(\mathscr{R}_{S}^{\prime}, \Delta \times \mathbb{N}^{\prime \times}\right) \longrightarrow\left(\mathbb{G m}_{S}, \Delta \times \mathbb{N}^{\prime \times}\right) .
$$

Étant donné un morphisme de $S$-schémas quasi-projectifs $f: X \longrightarrow \mathbb{A}_{S}^{1}$, on forme le diagramme commutatif à carrés cartésiens

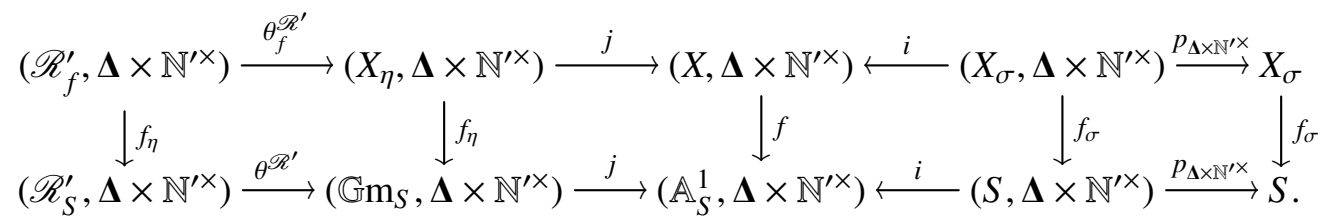

On définit alors le foncteur $\Psi_{f}^{\text {mod }}: \mathbf{D} A^{\text {ét }}\left(X_{\eta}, \Lambda\right) \longrightarrow \mathbf{D A}^{\text {ét }}\left(X_{\sigma}, \Lambda\right)$ par

$$
\Psi_{f}^{\bmod }=\left(p_{\Delta \times \mathbb{N}^{\prime \times}}\right)_{\sharp} i^{*} j_{*}\left(\theta_{f}^{\mathscr{R}^{\prime}}\right)_{*}\left(\theta_{f}^{\mathscr{R}^{\prime}}\right)^{*}\left(p_{\Delta \times \mathbb{N}^{\prime \times}}\right)^{*} .
$$

On obtient ainsi le système de spécialisation pseudo-monoïdal $\Psi^{\bmod }$ au-dessus de $\left(\mathbb{A}_{S}^{1}, j, i\right)$. Les foncteurs $\Psi_{f}^{\text {mod }}$ sont appelés les foncteurs « partie modéré du motif proche ». Par construction, on a une chaîne de morphismes évidents $\chi \longrightarrow \Upsilon \longrightarrow \Psi^{\text {mod }}$. On a le résultat suivant.

Theoreme 10.6 - Soient $S$ un schéma de base et $\Lambda$ un anneau de coefficients. On suppose que l'Hypothèse 7.3 est satisfaite. On se donne un morphisme de $S$-schémas quasi-projectifs $f$ : $X \longrightarrow \mathbb{A}_{S}^{1}$ tel que $X$ et $\left(X_{\sigma}\right)_{\text {red }}$ sont réguliers. On suppose que $f=u \cdot g^{e}$ avec $u \in \Gamma\left(X, \mathcal{O}^{\times}\right)$, $g \in \Gamma(X, \mathcal{O})$ un générateur de l'idéal de définition du sous-schéma fermé $\left(X_{\sigma}\right)_{\text {red }} \subset X$ et e $\in \mathbb{N}-\{0\}$. Soit d le plus grand diviseur de e qui soit dans $\mathbb{N}^{\prime \times}$ (i.e., qui soit inversible sur $S$ ). Il existe alors un isomorphisme canonique dans $\mathbf{D} \mathbf{A}^{\mathrm{e}}\left(X_{\sigma}, \Lambda\right)$ :

$$
\Psi_{f}^{\text {mod }} \Lambda_{X_{\eta}} \simeq\left\{X_{\sigma}[v] /\left(v^{d}-u\right) \rightarrow X_{\sigma}\right\}_{*} \Lambda .
$$

Demonstration On commence d'abord par des considérations générales. Pour $n \in \mathbb{N}^{\prime \times}$, on note $\kappa_{n}: \mathbb{N}^{\prime \times} \longrightarrow \mathbb{N}^{\prime \times}$ le foncteur qui envoie $m \in \mathbb{N}^{\prime \times}$ sur $m \cdot n$. On note aussi $\kappa_{n}$ l'endofoncteur id $\mathrm{i}_{\Delta} \times \kappa_{n}$ 
de $\Delta \times \mathbb{N}^{\prime \times}$. D'après [5, Lem. 3.5.5], le diagramme de $S$-schémas $\mathscr{R}_{S}^{\prime} \circ \kappa_{n}$ s'identifie canoniquement à $\mathscr{R}_{S}^{\prime}$. De plus, on a un carré commutatif

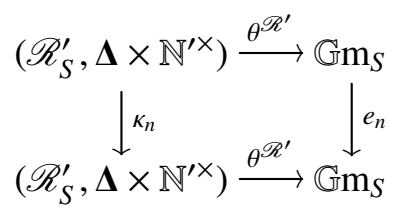

avec $e_{n}$ l'élévation à la puissance $n$ sur $\mathbb{G m}_{S}$. On note aussi $e_{n}$ l'élévation à la puissance $n$ sur $\mathbb{A}_{S}^{1}$. On pose alors $X_{n}=X \times_{\mathbb{A}_{S}^{1}, e_{n}} \mathbb{A}_{S}^{1}$ le changement de base du $\mathbb{A}_{S}^{1}$-schéma $X$ suivant $e_{n}$. La projection sur le second facteur fournit le morphisme $f_{n}: X_{n} \longrightarrow \mathbb{A}_{S}^{1}$. Comme dans [5, Prop. 3.5.9], le carré commutatif (98) induit un isomorphisme canonique $\Psi_{f_{n}}^{\text {mod }}\left(\operatorname{id}_{X} \times e_{n}\right)_{\eta}^{*} \simeq \Psi_{f}^{\text {mod }}$. Il suffit donc de construire un isomorphisme canonique

$$
\Psi_{f_{d}}^{\text {mod }} \Lambda \simeq\left\{X_{\sigma}[v] /\left(v^{d}-u\right) \rightarrow X_{\sigma}\right\}_{*} \Lambda .
$$

Notons $X_{d}^{\prime}$ la normalisation de $X_{d}, r: X_{d}^{\prime} \longrightarrow X_{d}$ le morphisme canonique et $f_{d}^{\prime}=f_{d} \circ r$. Puisque $\left(X_{d}\right)_{\eta}$ est étale au-dessus de $X_{\eta}$, il est normal. Il s'ensuit que $r_{\eta}$ est un isomorphisme. On a alors une chaîne d'isomorphismes canoniques

$$
\Psi_{f_{d}}^{m o d} \Lambda \simeq \Psi_{f_{d}}^{m o d} r_{\eta *} \Lambda \simeq r_{\sigma *} \Psi_{f_{d}^{\prime}}^{m o d} \Lambda .
$$

Par ailleurs, on a $X_{d} \simeq X[t] /\left(t^{d}-u \cdot g^{e}\right)$. Puisque $d$ divise $e$, un calcul immédiat montre que $X_{d}^{\prime} \simeq X[v] /\left(v^{d}-u\right)$. Le morphisme $X_{\sigma}[v] /\left(v^{d}-u\right) \rightarrow X_{\sigma}$ s'identifie ainsi à $r_{\sigma}$. Il est donc suffisant de construire un isomorphisme $\Psi_{f_{d}^{\prime}}^{\text {mod }} \Lambda \simeq \Lambda$. Pour cela, remarquons que $X[v] /\left(v^{d}-u\right)$ est étale sur $X$. Il s'ensuit que $X_{d}^{\prime}$ et $\left(\left(X_{d}^{\prime}\right)_{\sigma}\right)_{\text {red }}$ sont des schémas réguliers, et que le sous-schéma fermé $\left(\left(X_{d}^{\prime}\right)_{\sigma}\right)_{\text {red }} \subset X_{d}^{\prime}$ est défini par l'annulation de la fonction $g$ vue comme élément de $\Gamma\left(X_{d}^{\prime}, \mathcal{O}\right)$. De plus, nous avons la relation $f_{d}^{\prime}=t=v \cdot g^{e / d}$ dans $\Gamma\left(X_{d}^{\prime}, \mathcal{O}\right)$. Nous sommes donc ramenés au cas $d=1$ du théorème. Dans ce cas, on a un résultat plus précis décrit dans le Théorème 10.7 ci-dessous.

Theoreme 10.7 - Soient $S$ un schéma de base et $\Lambda$ un anneau de coefficients. On suppose que l'Hypothèse 7.3 est satisfaite. On se donne un morphisme de $S$-schémas quasi-projectifs $f$ : $X \longrightarrow \mathbb{A}_{S}^{1}$ tel que $X$ et $\left(X_{\sigma}\right)_{\text {red }}$ sont réguliers. On suppose que $f=u \cdot g^{e}$ avec $u \in \Gamma\left(X, O^{\times}\right)$, $g \in \Gamma(X, \mathcal{O})$ un générateur de l'idéal de définition du sous-schéma fermé $\left(X_{\sigma}\right)_{\text {red }} \subset X$ et e $\in \mathbb{N}-\{0\}$. Si e est premier à tout élément de $\mathbb{N}^{\prime \times}$, la composition de

$$
\Lambda_{X_{\sigma}} \rightarrow \chi_{f} \Lambda_{X_{\eta}} \rightarrow \Psi_{f}^{\text {mod }} \Lambda_{X_{\eta}}
$$

est un isomorphisme dans $\mathbf{D} \mathbf{A}^{\text {ét }}\left(X_{\sigma}, \Lambda\right)$.

Demonstration Remarquons d'abord que $e$ est inversible dans $\Lambda$ puisque aucun de ses diviseurs premiers n'est inversible sur $S$. On a un diagramme commutatif de diagrammes de $S$-schémas (cf. [5, Lem. 3.5.4])

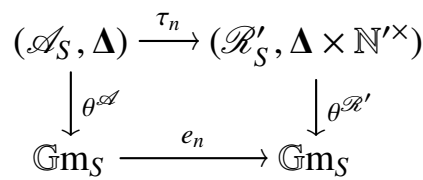

avec $\tau_{n}$ le morphisme déduit de l'inclusion $\Delta \simeq \Delta \times\{n\} \hookrightarrow \Delta \times \mathbb{N}^{\prime \times}$ et $e_{n}$ l'élévation à la puissance $n$ sur $\mathbb{G m}_{S}$. Comme dans la preuve ci-dessus, on pose $X_{n}=X \times_{\mathbb{A}_{S}^{1}, e_{n}} \mathbb{A}_{S}^{1}$ et on note $f_{n}: X_{n} \longrightarrow \mathbb{A}_{S}^{1}$ la projection sur le second facteur. Le carré commutatif (99) induit un morphisme $\Upsilon_{f_{n}}\left(\mathrm{id}_{X} \times e_{n}\right)_{\eta}^{*} \longrightarrow \Psi_{f}^{\text {mod }}$. De plus, $\Psi_{f}^{\text {mod }} \Lambda$ s'identifie à la colimite homotopique suivant $n \in \mathbb{N}^{\prime \times}$ des $\Upsilon_{f_{n}}\left(\mathrm{id}_{X} \times e_{n}\right)_{\eta}^{*} \Lambda$. Ainsi, il suffira de montrer que la composition de

$$
\Lambda_{\left(X_{n}\right)_{\sigma}} \longrightarrow \chi_{f_{n}} \Lambda_{\left(X_{n}\right)_{\eta}} \longrightarrow \Upsilon_{f_{n}} \Lambda_{\left(X_{n}\right)_{\eta}}
$$


est inversible.

Notons $X_{n}^{\prime}$ le normalisé de $X_{n}$ et $r: X_{n}^{\prime} \longrightarrow X$ le morphisme évident. On a $X_{n} \simeq X[t] /\left(t^{n}-u \cdot g^{e}\right)$. Il s'ensuit que $\left(X_{n}\right)_{\eta}$ est normal et que $r_{\eta}$ est inversible. Choisissons une relation de Bézout $a \cdot n+b \cdot e=$ 1 avec $(a, b) \in \mathbb{Z}^{2}$. Un calcul facile montre que $X_{n}^{\prime}=X[s] /\left(s^{n}-u^{b} \cdot g\right)$. Le morphisme $r$ envoie $t$ sur $u^{a} \cdot s^{e}$. En fait, le schéma $X[s] /\left(s^{n}-u^{b} \cdot g\right)$ est régulier puisque l'ouvert $\left(X_{n}\right)_{\eta} \simeq X\left[s, s^{-1}\right] /\left(s^{n}-u^{b} \cdot g\right)$ est régulier et son complémentaire est un diviseur de Cartier régulier. Ce diviseur de Cartier est défini par l'annulation de $s$ et il est canoniquement isomorphe à $\left(X_{\sigma}\right)_{\text {red }}$.

Il est à présent facile de conclure. En effet, on vient de voir que $r_{\eta}$ est un isomorphisme et que $r_{\sigma}$ est inversible à nil-immersions près. Il est donc suffisant de montrer que la composition de

$$
\Lambda_{\left(X_{n}^{\prime}\right)_{\sigma}} \longrightarrow \chi_{f_{n}^{\prime}} \Lambda_{\left(X_{n}^{\prime}\right)_{\eta}} \longrightarrow \Upsilon_{f_{n}^{\prime}} \Lambda_{\left(X_{n}^{\prime}\right)_{\eta}}
$$

est inversible avec $f_{n}^{\prime}=f_{n} \circ r$. Or, le morphisme $f_{n}^{\prime}: X_{n}^{\prime} \longrightarrow \mathbb{A}_{S}^{1}$ satisfait aux conditions d'application du Théorème 10.2.

À partir de maintenant, $S=\operatorname{Spec}(R)$ sera le spectre d'un anneau de valuation discrète excellent et hensélien $R$ d'idéal maximal $\mathfrak{m}$. On fixe une uniformisante $\pi \in \mathfrak{m}$ qui définit un morphisme $\pi: S \longrightarrow \mathbb{A}_{S}^{1}$. On note $\eta$ et $\sigma$ au lieu de $S_{\eta}$ et $S_{\sigma}$ de sorte que $\eta$ est le point ouvert de $S$ et $\sigma$ est son point fermé.

Definition 10.8 - Soit $f: X \longrightarrow S$ un $S$-schéma quasi-projectif. Lorsque cela n'entraîne pas confusion, on notera $\Upsilon_{f}$ et $\Psi_{f}^{\text {mod }}: \mathbf{D A}^{\mathrm{e}}\left(X_{\eta}, \Lambda\right) \longrightarrow \mathbf{D A}^{\text {et }}\left(X_{\sigma}, \Lambda\right)$ au lieu de $\Upsilon_{\pi \circ f}$ et $\Psi_{\pi \circ f}^{\text {mod }}$ les foncteurs «motif proche partiel » définis ci-dessus. Il nous arrivera aussi de noter $\Upsilon_{X}$ et $\Psi_{X}^{\text {mod }}$ ces foncteurs.

Le résultat suivant montre que nos foncteurs $\Upsilon_{f}$ et $\Psi_{f}^{\text {mod }}$ sont raisonnables lorsque les bonnes conditions sont assurées.

Theoreme 10.9 - Supposons donné un $S$-schéma quasi-projectif $f: X \longrightarrow S$.

(i) Le foncteur $\Psi_{f}^{\bmod }: \mathbf{D A}^{\mathrm{e} t}\left(X_{\eta}, \Lambda\right) \longrightarrow \mathbf{D A}^{\mathrm{e}}\left(X_{\sigma}, \Lambda\right)$ préserve les objets constructibles lorsque l'Hypothèse 7.3 est satisfaite.

(ii) Le foncteur $\Upsilon_{f}: \mathbf{D A}^{\mathrm{e}}{ }^{\mathrm{t}}\left(X_{\eta}, \Lambda\right) \longrightarrow \mathbf{D A}^{\mathrm{e} t}\left(X_{\sigma}, \Lambda\right)$ préserve les objets constructibles lorsque $\Lambda$ est une $\mathbb{Q}$-algèbre.

Demonstration On démontre uniquement la propriété (i). La preuve de (ii) est la même en plus simple. En effet, pour (i) nous aurons besoin d'utiliser la version $\ell$-primaire due à Gabber de la résolution des singularités par altérations de de Jong [29] alors que pour (ii) la version originale de de Jong est suffisante.

Il suffit de montrer que $\Psi_{f}^{\text {mod }} \Lambda$ est constructible pour tous les $S$-schémas quasi-projectifs $X$. En effet, pour $X$ fixé, la Proposition 3.19 et [4, Lem. 2.2.23] entraînent que la catégorie $\mathbf{D} \mathbf{A}^{\text {ét }}\left(X_{\eta}, \Lambda\right)$ est compactement engendrée par les motifs de la forme $\left(g_{\eta}\right)_{*} \Lambda(n)$ avec $g: Y \longrightarrow X$ un $S$-morphisme projectif et $n \in \mathbb{Z}$. (Ces objets sont constructibles d'après la Proposition 8.5, (b).) Il suffit donc de prouver que $\Psi_{f}^{\bmod }\left(g_{\eta}\right)_{*} \Lambda$ est constructible. Or, le morphisme $\Psi_{f}^{\bmod }\left(g_{\eta}\right)_{*} \longrightarrow\left(g_{\sigma}\right)_{*} \Psi_{f \circ g}^{\bmod }$ est inversible et $\left(g_{\sigma}\right)_{*}$ préserve les objets constructibles. Il suffit donc de prouver que $\Psi_{f \circ g}^{\bmod } \Lambda$ est constructible, ce qui établit la réduction souhaitée.

Pour montrer que $\Psi_{f}^{\text {mod }} \Lambda$ est constructible nous allons raisonner par induction sur la dimension de Krull de la fibre générique $X_{\eta}$. Supposons d'abord que cette dimension est nulle. On ne restreint pas la généralité en remplaçant $X$ par l'adhérence de $X_{\eta}$ et ensuite par son normalisé. On peut donc supposer que $X$ est le spectre d'un anneau de valuation discrète hensélien fini sur $S$. On peut alors utiliser le Théorème 10.6 pour conclure.

Supposons maintenant que $\operatorname{krdim}\left(X_{\eta}\right) \geq 1$. Fixons un nombre premier $\ell$ inversible sur $S$. D'après le Corollaire 8.4, il suffit de montrer qu'il existe un entier $e \in \mathbb{N}$ premier à $\ell$ tel que $\Psi_{f}^{\bmod }\left(\Lambda\left[e^{-1}\right]\right)$ est constructible dans $\mathbf{D A} \mathbf{A}^{\mathrm{et}}\left(X_{\sigma}, \Lambda\left[e^{-1}\right]\right)$. Un dévissage standard (utilisant l'hypothèse d'induction) nous ramène au cas où $X$ est intègre et plat sur $S$. D'après [25, Expo. X, Th. 2.4], 
il existe un carré commutatif

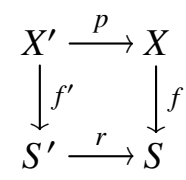

avec $S^{\prime}$ le spectre d'un anneau de valuation discrète hensélien, $X^{\prime}$ un schéma intègre qui est à réduction semi-stable au-dessus de $S^{\prime}$ (au sens affaibli de [5, Déf. 3.3.33]), $r$ un morphisme fini et $p$ une altération de degré générique premier à $\ell$. La condition de semi-stabilité sur $X^{\prime}$ est la suivante. Localement pour la topologie étale, $X^{\prime}$ est isomorphe à $S^{\prime}\left[t_{0}, \cdots, t_{n}\right] /\left(t_{0}^{a_{0}} \cdots t_{r}^{a_{r}}-\pi^{\prime}\right)$ avec $\pi^{\prime}$ une uniformisante de $S^{\prime}$ (cf. [5, Lem. 3.3.37 et 3.3.38]). Dans la suite, on prendra pour $e$ le degré générique de $p$. Quitte à remplacer $\Lambda$ par $\Lambda\left[e^{-1}\right]$, on peut supposer que $e$ est inversible dans $\Lambda$. C'est ce qu'on fera dans la suite.

Pour démontrer que $\Psi_{f}^{\text {mod }} \Lambda$ est constructible, il suffit par l'hypothèse d'induction de montrer que $\Psi_{f}^{\text {mod }} u ! \Lambda$ est inversible avec $u: U \hookrightarrow X_{\eta}$ l'inclusion d'un ouvert dense au-dessus duquel $p$ est la composition d'un revêtement étale suivi d'un morphisme fini totalement inséparable. Notons $U^{\prime}=p^{-1}(U), u^{\prime}: U^{\prime} \hookrightarrow X_{\eta}^{\prime}$ l'inclusion évidente et $q: U^{\prime} \longrightarrow U$ le morphisme déduit de $p$. D'après [4, Lem. 2.1.165] et puisque $e$ est supposé inversible dans $\Lambda$, le morphisme $\Lambda_{U} \longrightarrow q_{*} \Lambda_{U^{\prime}}$ admet une rétraction. Vue la chaîne d'isomorphismes

$$
\left(p_{\sigma}\right)_{*} \Psi_{f \circ p}^{\text {mod }} u_{!}^{\prime} \Lambda_{U^{\prime}} \simeq \Psi_{f}^{\text {mod }}\left(p_{\eta}\right)_{*} u_{!}^{\prime} \Lambda_{U^{\prime}} \simeq \Psi_{f}^{\text {mod }}\left(p_{\eta}\right) ! u_{!}^{\prime} \Lambda_{U^{\prime}} \simeq \Psi_{f}^{\text {mod }} u ! q ! \Lambda_{U^{\prime}} \simeq \Psi_{f}^{\text {mod }} u ! q_{*} \Lambda_{U^{\prime}}
$$

on déduit que $\Psi_{f}^{m o d} u_{!} \Lambda$ est isomorphe à un facteur direct de $\left(p_{\sigma}\right)_{*} \Psi_{f \circ p}^{m o d} u_{!}^{\prime} \Lambda$. Il est donc suffisant de montrer que $\Psi_{f \circ p}^{m o d} u_{!}^{\prime} \Lambda$ est constructible. En utilisant une deuxième fois l'hypothèse de récurrence, on se ramène à prouver que $\Psi_{r \circ f^{\prime}}^{\text {mod }} \Lambda$ est constructible. Cette propriété est locale pour la topologie étale sur $X_{\sigma}^{\prime}$. Le résultat recherché est maintenant une conséquence de [5, Th. 3.3.48] et du cas d'un $S$-schéma fini, i.e., celui où la dimension de Krull de la fibre générique est nulle. (Pour se débarasser dans [5, Th. 3.3.48] du cas des $b_{n}^{m}$, on utilise que, modulo un revêtement pseudogaloisien de degré inversible dans $\Lambda$ et localement pour la topologie étale, le morphisme $b_{n}^{m}$ : $S^{\prime}\left[U, U^{-1}\right][T] /\left(T^{n}-U^{m} \cdot \pi^{\prime}\right) \longrightarrow S^{\prime}$ est isomorphe à $b_{n}: S^{\prime}[T] /\left(T^{n}-\pi^{\prime}\right) \longrightarrow S^{\prime}$.)

Pour faire le lien avec les constructions correspondantes en cohomologie étale, nous avons besoin d'introduire un autre système de spécialisation $\widehat{\chi}$. Il est défini à partir du diagramme de $S$-schémas $\left(\mathscr{E}_{S}^{\prime \prime}, \mathbb{N}^{\prime \times}\right)$. (Rappelons que ce dernier s'identifie à la restriction de $\mathscr{R}_{S}^{\prime}$ à $\underline{\mathbf{0}} \times \mathbb{N}^{\prime \times}$.) Étant donné un morphisme de $S$-schémas quasi-projectifs $f: X \longrightarrow \mathbb{A}_{S}^{1}$, on forme le diagramme commutatif à carrés cartésiens

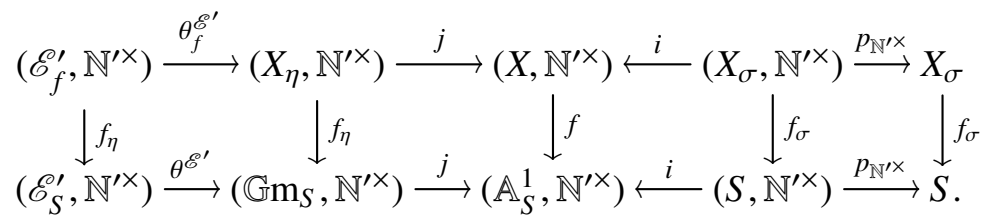

On définit alors le foncteur $\widehat{\chi}_{f}: \mathbf{D A}^{\text {ét }}\left(X_{\eta}, \Lambda\right) \longrightarrow \mathbf{D A}^{\text {ét }}\left(X_{\sigma}, \Lambda\right)$ par

$$
\widehat{\chi}_{f}=\left(p_{\mathbb{N}^{\prime x}}\right)_{\sharp} i^{*} j_{*}\left(\theta_{f}^{\mathscr{E}^{\prime \prime}}\right)_{*}\left(\theta_{f}^{\mathscr{E}^{\prime}}\right)^{*} p_{\mathbb{N}^{\prime} x}^{*} .
$$

Par construction, on a une suite de morphismes de systèmes de spécialisation pseudo-monoïdaux $\chi \longrightarrow \widehat{\chi} \longrightarrow \Psi^{\text {mod }}$. Comme avant, le choix de l'uniformisante $\pi \in \mathrm{m}$ permet de restreindre le système de spécialisation pseudo-monoïdal $\widehat{\chi}$ au-dessus de la base $(S, j: \eta \hookrightarrow S, i: \sigma \hookrightarrow S)$. Ainsi, si $f: X \longrightarrow S$ est un $S$-schéma quasi-projectif, nous écrirons $\widehat{\chi}_{f}$ au lieu de $\widehat{\chi}_{\pi \circ f}$.

Theoreme 10.10 - Supposons que l'anneau de coefficients $\Lambda$ est de torsion et qu'il satisfait à l'Hypothèse 7.3. Soit $f: X \longrightarrow S$ un $S$-schéma quasi-projectif. Alors, le morphisme $\widehat{\chi}_{f} \longrightarrow \Psi_{f}^{\text {mod }}$ est un isomorphisme. 
Demonstration La preuve que nous présenterons dépend, elle aussi, de la version $\ell$-primaire due à Gabber de la résolution des singularités par altérations de de Jong [29].

Il suffit de montrer que $\widehat{\chi}_{f} \Lambda \longrightarrow \Psi_{f}^{\text {mod }} \Lambda$ est inversible pour tous les $S$-schémas quasi-projectifs $X$. En effet, pour $X$ fixé, la Proposition 3.19 et [4, Lem. 2.2.23] entraînent que $\mathbf{D A}^{\text {ét }}\left(X_{\eta}, \Lambda\right)$ est compactement engendrée par les motifs de la forme $\left(g_{\eta}\right)_{*} \Lambda(n)$ avec $g: Y \longrightarrow X$ un $S$-morphisme projectif et $n \in \mathbb{Z}$. Puisque les foncteurs $\widehat{\chi}_{f}$ et $\Psi_{f}^{\text {mod }}$ commutent aux sommes infinies, il est donc suffisant de montrer que $\widehat{\chi}_{f}\left(g_{\eta}\right)_{*} \Lambda \longrightarrow \Psi_{f}^{\bmod }\left(g_{\eta}\right)_{*} \Lambda$ est inversible. Puisque $g$ est projectif, les morphismes $\widehat{\chi}_{f}\left(g_{\eta}\right)_{*} \longrightarrow\left(g_{\sigma}\right)_{*} \widehat{\chi}_{f \circ g}$ et $\Psi_{f}^{\bmod }\left(g_{\eta}\right)_{*} \longrightarrow\left(g_{\sigma}\right)_{*} \Psi_{f \circ g}^{\text {mod }}$ sont inversibles. Ceci nous ramène à prouver que $\widehat{\chi}_{f \circ g} \Lambda \longrightarrow \Psi_{f \circ g}^{\text {mod }} \Lambda$ est inversible, ce qui établit la réduction souhaitée.

On ne restreint pas la généralité en supposant que $\Lambda$ est de caractéristique une puissance d'un nombre premier $\ell$ inversible sur $S$. On montrera que $\widehat{\chi}_{f} \Lambda \longrightarrow \Psi_{f}^{\text {mod }} \Lambda$ est un isomorphisme par induction sur la dimension de Krull de $X_{\eta}$. On divise la preuve en deux parties. Dans la première, nous établirons le cas où $\operatorname{krdim}\left(X_{\eta}\right)=0$.

Partie A : On suppose ici que $X_{\eta}$ est de dimension nulle. On ne restreint pas la généralité en remplaçant $X$ par l'adhérence de $X_{\eta}$ et puis par son normalisé. Autrement dit, on peut supposer que $X=T$ est le spectre d'un anneau de valuation discrète $Q$ fini sur $R$. Soit $\varpi$ une uniformisante de $Q$ et supposons que $\pi=u \cdot \varpi^{e}$ avec $u$ inversible dans $Q$.

Soit $d$ le plus grand diviseur de $e$ qui est dans $\mathbb{N}^{\prime \times}$. D'après le Théorème 10.6, on a un isomorphisme canonique :

$$
\Psi_{T}^{\bmod }(\Lambda) \simeq\left\{T_{\sigma}[v] /\left(v^{d}-u\right) \rightarrow T_{\sigma}\right\}_{*} \Lambda .
$$

Nous prouverons une formule similaire pour $\widehat{\chi}_{S}, \Lambda$ et nous laisserons au lecteur le soin de vérifier que modulo ces identifications, le morphisme qui nous intéresse est l'identité.

En remplaçant « $\mathscr{R}^{\prime}$ » et « $\Psi$ mod $»$ par « $\mathscr{E}^{\prime}$ » et « $\widehat{\chi}$ » dans la preuve du Théorème 10.6 on obtient une réduction au cas $d=1$. Pour traiter ce cas, nous montrerons que la composition de

$$
\Lambda_{T_{\sigma}} \longrightarrow \chi_{T} \Lambda_{T_{\eta}} \longrightarrow \widehat{\chi}_{T} \Lambda_{T_{\eta}}
$$

est un isomorphisme.

Pour $n \in \mathbb{N}^{\prime \times}$, on pose $T_{n}^{\prime}=T\left[\varpi^{1 / n}\right]$. Vu que $n$ et $e$ sont supposés premiers entre-eux, $T_{n}^{\prime}$ est le normalisé de $T \times_{S} S\left[\pi^{1 / n}\right]$. De plus, on a une identification canonique $\left(T_{n}^{\prime}\right)_{\sigma} \simeq T_{\sigma}$. Modulo cette identification, la composition de (101) est donnée par :

$$
\Lambda \longrightarrow \text { hocolim }_{n \in \mathbb{N}^{\prime \prime}} \chi_{T_{n}^{\prime}} \Lambda \text {. }
$$

Or, puisque $T_{n}^{\prime}$ est le spectre d'un anneau de valuation discrète, on a $\chi_{T_{n}^{\prime}} \Lambda_{\left(T_{n}^{\prime}\right)_{\eta}}=i^{*} j_{*} \Lambda_{\left(T_{n}^{\prime}\right)_{\eta}} \simeq$ $\Lambda_{T_{\sigma}} \oplus \Lambda_{T_{\sigma}}(-1)[-1]$. D'après la Proposition 10.3, le morphisme $\chi_{T_{m}^{\prime}} \Lambda \longrightarrow \chi_{T_{n}^{\prime}} \Lambda$, pour $m$ divisant $n$, est donné par la matrice

$$
\left(\begin{array}{cc}
1 & 0 \\
0 & n m^{-1}
\end{array}\right)
$$

Puisque $\ell \in \mathbb{N}^{\prime \times}$ et qu'une puissance de $\ell$ est nulle dans $\Lambda$, on voit aussitôt que (102) est inversible. Partie $B$ : On suppose ici que $\operatorname{krdim}\left(X_{\eta}\right) \geq 1$. Par un dévissage standard, on peut supposer que $X$ est intègre et plat sur $S$. D'après [25, Expo. X, Th. 2.4], il existe un carré commutatif

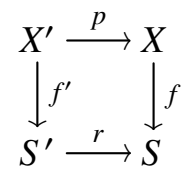

avec $S^{\prime}$ le spectre d'un anneau de valuation discrète hensélien, $X^{\prime}$ un schéma intègre qui est à réduction semi-stable au-dessus de $S^{\prime}$ (au sens affaibli de [5, Déf. 3.3.33]), $r$ un morphisme fini et $p$ une altération de degré générique premier à $\ell$. La condition de semi-stabilité sur $X^{\prime}$ est la suivante. Localement pour la topologie étale, $X^{\prime}$ est isomorphe à $S^{\prime}\left[t_{0}, \cdots, t_{n}\right] /\left(t_{0}^{a_{0}} \cdots t_{r}^{a_{r}}-\pi^{\prime}\right)$ avec $\pi^{\prime}$ une uniformisante de $S^{\prime}$ (cf. [5, Lem. 3.3.37 et 3.3.38]). 
Pour démontrer que $\widehat{\chi}_{f} \Lambda \longrightarrow \Psi_{f}^{\text {mod }} \Lambda$ est inversible, il suffit par l'hypothèse d'induction de montrer que $\widehat{\chi}_{f} u_{!} \Lambda \longrightarrow \Psi_{f}^{\text {mod }} u_{!} \Lambda$ est inversible avec $u: U \hookrightarrow X_{\eta}$ l'inclusion d'un ouvert dense audessus duquel $p$ est la composition d'un revêtement étale suivi d'un morphisme fini totalement inséparable. Notons $U^{\prime}=p^{-1}(U), u^{\prime}: U^{\prime} \hookrightarrow X_{\eta}^{\prime}$ l'inclusion évidente et $q: U^{\prime} \longrightarrow U$ le morphisme déduit de $p$. D'après [4, Lem. 2.1.165] et puisque le degré de $q$ est inversible dans $\Lambda$, le morphisme $\Lambda_{U} \longrightarrow q_{*} \Lambda_{U^{\prime}}$ admet une rétraction. On a un carré commutatif

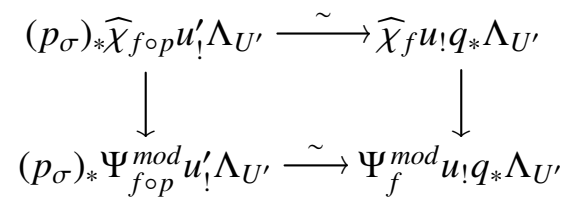

où les flèches horizontales sont inversibles. Il est donc suffisant de montrer que le morphisme $\widehat{\chi}_{f \circ p} u_{!}^{\prime} \Lambda \longrightarrow \Psi_{f \circ p}^{\text {mod }} u_{!}^{\prime} \Lambda$ est inversible. En utilisant une deuxième fois l'hypothèse de récurrence, on se ramène à prouver que $\widehat{\chi}_{f \circ p} \Lambda \longrightarrow \Psi_{f \circ p}^{\text {mod }} \Lambda$ est inversible. Or, cette propriété est locale pour la topologie étale sur $X_{\sigma}^{\prime}$. Le résultat recherché est alors une conséquence de [5, Th. 3.3.45] et du cas d'un $S$-schéma fini, i.e., celui où la dimension de Krull de la fibre générique est nulle. (Pour se débarasser dans [5, Th. 3.3.45] du cas des $b_{n}^{m}$, on utilise que, modulo un revêtement pseudo-galoisien de degré inversible dans $\Lambda$ et localement pour la topologie étale, le morphisme $b_{n}^{m}: S^{\prime}\left[U, U^{-1}\right][T] /\left(T^{n}-U^{m} \cdot \pi^{\prime}\right) \longrightarrow S^{\prime}$ est isomorphe à $\left.b_{n}: S^{\prime}[T] /\left(T^{n}-\pi^{\prime}\right) \longrightarrow S^{\prime}.\right)$

Rappelons que $S=\operatorname{Spec}(R)$ avec $R$ un anneau local hensélien de corps de fraction $K$. On note $\widetilde{R}$ la normalisation de $R$ dans l'extension $\widetilde{K}=K\left[\pi^{1 / n} \mid n \in \mathbb{N}^{\prime \times}\right]$ de $K$. On pose alors $\widetilde{\eta}=\operatorname{Spec}(\widetilde{K})$ et $\widetilde{S}=\operatorname{Spec}(\widetilde{R})$. Étant donné un $S$-schéma $f: X \longrightarrow S$, on forme le diagramme de schémas à carrés cartésiens

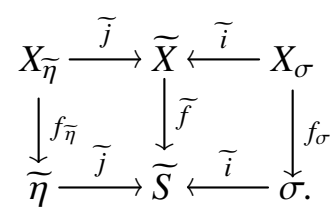

Soit $J \subset \Lambda$ un idéal tel que $\Lambda / J$ est de caractéristique non nulle et inversible sur $S$. On définit alors des foncteurs

$$
\Psi_{f}^{\text {mod }}: \mathbf{D}^{\text {ét }}\left(X_{\eta}, \Lambda / J\right) \longrightarrow \mathbf{D}^{\text {ét }}\left(X_{\sigma}, \Lambda / J\right) \quad \text { et } \quad \Psi_{f}^{\text {mod }}: \mathbf{D}^{\text {ét }}\left(X_{\eta}, \Lambda / J^{*}\right) \longrightarrow \mathbf{D}^{\text {ét }}\left(X_{\sigma}, \Lambda / J^{*}\right)
$$

par la formule $\Psi_{f}^{\text {mod }}(A)=\widetilde{i}^{*} \widetilde{j}_{*} A_{\widetilde{\eta}}$. C'est les foncteurs « cycles proches modérés » en cohomologie étale. Il est facile de voir que le second foncteur $\Psi_{f}^{\bmod }$ envoie la sous-catégorie $\hat{\mathbf{D}}^{\text {ét }}\left(X_{\eta}, \Lambda_{J}\right)$ dans $\hat{\mathbf{D}}^{\text {ét }}\left(X_{\sigma}, \Lambda_{J}\right)$ induisant ainsi un foncteur « cycles proches modérés »

$$
\Psi_{f}^{\bmod }: \hat{\mathbf{D}}^{\mathrm{ét}}\left(X_{\eta}, \Lambda_{J}\right) \longrightarrow \hat{\mathbf{D}}^{\mathrm{ét}}\left(X_{\sigma}, \Lambda_{J}\right) .
$$

Le résultat suivant est immédiat.

Theoreme 10.11 - On suppose que l'Hypothèse 6.5 est satisfaite. Soit $f: X \longrightarrow S$ un $S$-schéma quasi-projectif. Il existe alors des faces carrées inversibles (de foncteurs pseudo-monö̈daux)

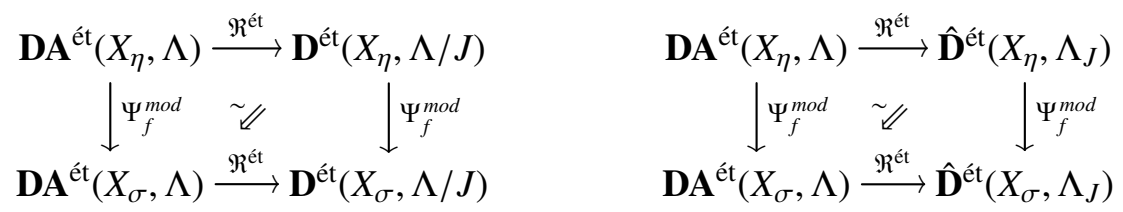

qui sont compatibles aux morphismes de changement de base associés aux $S$-morphismes. 
Demonstration On traite uniquement le cas des coefficients dans $\Lambda / J$. Vus le Théorème 10.10 et la construction de la réalisation étale, on est ramené à construire des faces carrées inversibles

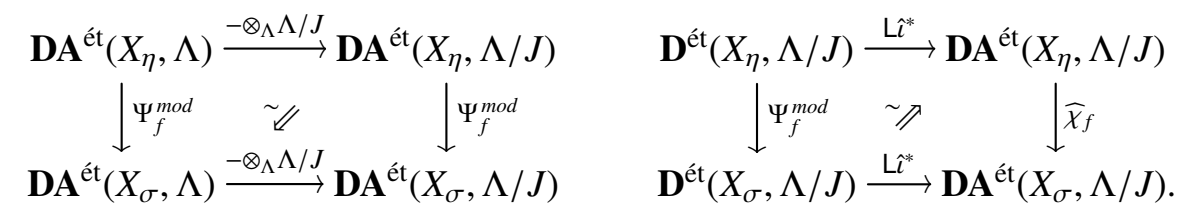

La première face ci-dessus est la composition de 2-morphismes de type $\gamma$ et $\xi$, comme dans la Proposition 6.2, associés à des morphismes de diagrammes (au lieu de morphismes de schémas), et d'un isomorphisme de commutation de $-\otimes_{\Lambda} \Lambda / J$ avec la colimite homotopique suivant $\Delta \times \mathbb{N}^{\prime \times}$. Il découle aussitôt du Théorème 6.3 que cette face carrée est inversible.

La deuxième face ci-dessus est obtenue directement à partir des constructions. En effet, remarquons que $\Psi_{f}^{\text {mod }}(A)=\widetilde{i^{*}} \widetilde{j}_{*} A_{\tilde{\eta}}$ est canoniquement isomorphe à $i^{*} j_{*} \widetilde{\theta}_{f *} \widetilde{\theta}_{f}^{*} A$ avec $\widetilde{\theta}: \widetilde{\eta} \longrightarrow \eta$ le morphisme canonique et $\widetilde{\theta}_{f}: X_{\widetilde{\eta}} \longrightarrow X_{\eta}$ son changement de base suivant $f_{\eta}$. Par ailleurs, $\widetilde{\theta}_{f *} \widetilde{\theta}_{f}^{*} A$ est canoniquement isomorphe à $\left(p_{\mathbb{N}^{\prime}}\right)_{\sharp}\left(\theta_{f}^{\mathscr{E}^{\prime}}\right)_{*}\left(\theta_{f}^{\mathscr{E}^{\prime}}\right)^{*}\left(p_{\mathbb{N}^{\prime} \times}\right)^{*} A$. Tout ceci est valable aussi bien dans les catégories $\mathbf{D} A^{\text {ét }}(-, \Lambda / J)$ que dans les catégories $\mathbf{D}^{\text {ét }}(-, \Lambda / J)$ et c'est compatible aux équivalences $\mathrm{L} \hat{\imath}^{*}$. Pour conclure on utilise encore que le foncteur $j_{*}$ commute aux sommes directes infinies et donc aussi aux colimites homotopiques suivant $\mathbb{N}^{\prime x}$.

En tensorisant par $\mathbb{Q}$ et en passant aux enveloppes pseudo-abléliennes, on obtient également le résultat suivant.

Theoreme 10.12 - On suppose que l'Hypothèse 7.3 est satisfaite. Soit $f: X \longrightarrow S$ un $S$-schéma quasi-projectif. Il existe alors une face carrée inversible (de foncteurs pseudo-monoïdaux)

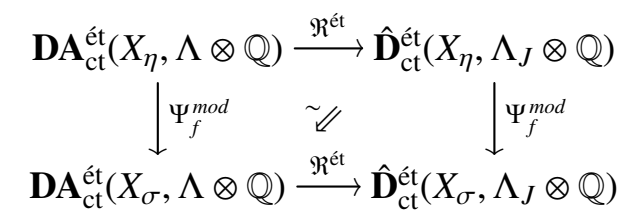

qui est compatible aux morphismes de changement de base associés aux $S$-morphismes.

Demonstration On utilise que les foncteurs $\Psi_{f}^{\bmod }$ préservent les objets constructibles.

On arrive maintenant à la construction des foncteurs «motif proche total » dans le contexte motivique et la comparaison avec leurs analogues en cohomologie étale. Lorsque $S$ est d'égale caractéristique nulle, les foncteurs $\Psi_{f}^{\text {mod }}$ définis ci-dessus sont les foncteurs « motif proche total ». On les notera simplement $\Psi_{f}$.

Dans la suite, on supposera que la caractéristique résiduelle de $S$ est non nulle et on la notera $p$. Fixons une extension séparablement close $K^{s e p} / K$ et notons $K^{n r}$ l'extension maximale non ramifiée de $K$ contenue dans $K^{\text {sep }}$. Le groupe de Galois de $K^{s e p} / K^{n r}$ s'insère dans une suite exacte de groupes pro-finis

$$
1 \longrightarrow \operatorname{Gal}_{p}\left(K^{\text {sep }} / K^{n r}\right) \longrightarrow \operatorname{Gal}\left(K^{\text {sep }} / K^{n r}\right) \stackrel{\phi}{\longrightarrow} \widehat{\mathbb{Z}}^{\prime}(1) \longrightarrow 1
$$

où $\widehat{\mathbb{Z}}^{\prime}(1)=\lim _{n \in \mathbb{N}^{\prime x}} \mu_{n}\left(K^{\text {sep }}\right)$, qu'on peut décomposer en $\prod_{\ell \neq p} \mathbb{Z}_{\ell}(1)=\prod_{\ell \neq p} \lim _{v \in \mathbb{N}} \mu_{\ell^{v}}\left(K^{\text {sep }}\right)$, et $\phi$ l'homomorphisme qui envoie $\sigma \in \operatorname{Gal}\left(K^{s e p} / K^{n r}\right)$ sur $\left(\sigma\left(\pi^{1 / r}\right) / \pi^{1 / r}\right)_{r \in \mathbb{N}^{\prime x}}$. Le noyau de $\phi$, noté $\operatorname{Gal}_{p}\left(K^{\text {sep }} / K^{n r}\right)$, est un pro- $p$-groupe. C'est l'unique $p$-sous-groupe de Sylow de $\operatorname{Gal}\left(K^{\text {sep }} / K^{n r}\right)$. D'après le théorème de Schur-Zassenhaus (cf. [47, Th. 8.10]), le morphisme $\phi$ est scindé. On fixe une section $\delta: \widehat{\mathbb{Z}}^{\prime}(1) \longrightarrow \operatorname{Gal}\left(K^{\text {sep }} / K^{n r}\right)$ et on note $M_{\delta} \subset K^{\text {sep }}$ le sous-corps des invariants par $\delta\left(\widehat{\mathbb{Z}}^{\prime}(1)\right)$. Le corps $M_{\delta}$ est une union filtrante d'extensions finies de $K^{n r}$ de degré une puissance de $p$.

Theoreme 10.13 - On suppose que l'Hypothèse 7.3 est satisfaite. Soient $f: X \longrightarrow S$ un $S$ schéma quasi-projectif et $A \in \mathbf{D A}^{\mathrm{e}}\left(X_{\eta}, \Lambda\right)$ un motif constructible. Il existe alors une extension finie 
$L / K$ contenue dans $M_{\delta}$ telle que pour toute extension finie $L^{\prime} / K$, contenue dans $M_{\delta}$ et contenant $L$, la suite de l'énoncé est vérifiée. Notons $T$ et $T^{\prime}$ les spectres des normalisés de $R$ dans $L$ et $L^{\prime}$, et $t: T \longrightarrow S$ et $u: T^{\prime} \longrightarrow T$ les morphismes évidents. Notons aussi $t: X \times_{S} T \longrightarrow X$ et $u: X \times_{S} T^{\prime} \longrightarrow X \times_{S} T$ les morphismes déduis de tet u par changement de base suivant $f$. Alors, le morphisme canonique

$$
u_{\sigma}^{*} \Psi_{X \times_{S} T}^{\bmod T} t_{\eta}^{*} A \longrightarrow \Psi_{X \times_{S} T^{\prime}}^{\bmod } u_{\eta}^{*} t_{\eta}^{*} A
$$

est un isomorphisme dans $\mathbf{D A}^{\text {ét }}\left(\left(X \times_{S} T^{\prime}\right)_{\sigma}, \Lambda\right)$.

Demonstration Il suffit de montrer le théorème pour $A=\Lambda_{X_{\eta}}$ et pour tous les $S$-schémas quasiprojectifs $X$. En effet, pour $X$ fixé, la Proposition 3.19 et [4, Lem. 2.2.23] entraînent que la catégorie $\mathbf{D A}{ }^{\text {ét }}\left(X_{\eta}, \Lambda\right)$ est compactement engendrée par les motifs de la forme $\left(g_{\eta}\right)_{*} \Lambda(n)$ avec $g: Y \longrightarrow X$ un $S$-morphisme projectif et $n \in \mathbb{Z}$. (Ces objets sont constructibles d'après la Proposition 8.5 , (b).) Il suffit donc de prouver le théorème pour $A=\left(g_{\eta}\right)_{*} \Lambda$. Or, les morphismes $\Psi_{X \times_{S} T}^{\bmod }\left(g_{\eta}\right)_{*} \longrightarrow\left(g_{\sigma}\right)_{*} \Psi_{Y \times_{S} T}^{\bmod }$ et $\Psi_{X \times_{S} T^{\prime}}^{\bmod }\left(g_{\eta}\right)_{*} \longrightarrow\left(g_{\sigma}\right)_{*} \Psi_{Y \times_{S} T^{\prime}}^{\bmod }$ sont inversibles pour $T$ et $T^{\prime}$ comme dans l'énoncé. (Ici, on a encore noté $g$ les changements de base de $g$ par $t$ et $t \circ u$.) Il suffit donc de prouver le théorème pour $\Lambda_{Y_{\eta}}$ et $f \circ g: Y \longrightarrow S$, ce qui établit la réduction souhaitée.

Pour montrer le théorème dans le cas $A=\Lambda_{X_{\eta}}$, on raisonne par récurrence sur la dimension de Krull de $X_{\eta}$. On suppose d'abord que cette dimension est nulle. On ne restreint pas la généralité en remplaçant $X$ par l'adhérence de $X_{\eta}$ et ensuite par son normalisé. On peut donc supposer que $X=S^{\prime}$ est le spectre d'un anneau de valuation discrète hensélien $R^{\prime}$ fini sur $R$. Notons $K^{\prime}$ le corps des fractions de $R^{\prime}$. On peut trouver une sous-extension finie $L / K$ de $M_{\delta}$ telle que les algèbres $K^{\prime} \otimes_{K} L$ et $K^{\prime} \otimes_{K} M_{\delta}$ ont les mêmes nombres d'idéaux premiers. Notons $E$ et $E^{\prime}$ les normalisés des schémas $T \times_{S} S^{\prime}$ et $T^{\prime} \times_{S} S^{\prime}$ respectivement, et $v: E^{\prime} \longrightarrow E$ le morphisme induit. Les corps résiduels des composantes connexes de $E$ (resp. $E^{\prime}$ ) sont tous isomorphes au corps résiduel de $T$ (resp. $T^{\prime}$ ). Il suffit alors de montrer que $v_{\sigma}^{*} \Psi_{E}^{\text {mod }} \Lambda \longrightarrow \Psi_{E^{\prime}}^{\bmod } \Lambda$ est inversible. Par construction, $E=\bigsqcup_{i \in I} E_{i}$ et $E^{\prime}=\bigsqcup_{i \in I} E_{i}^{\prime}$ où $I$ est un ensemble fini, $E_{i}$ et $E_{i}^{\prime}$ sont les spectres d'anneaux de valuations discrètes finis sur $S$, et $v$ est le coproduit de morphismes $v_{i}: E_{i}^{\prime} \longrightarrow E_{i}$ qui sont une composition d'un morphisme non ramifié suivi d'un morphisme totalement ramifié de degré générique une puissance de $p$. Le résultat recherché découle maintenant du Théorème 10.7.

Supposons maintenant que $\operatorname{krdim}\left(X_{\eta}\right) \geq 1$. Fixons un nombre premier $\ell$ inversible sur $S$. Nous montrerons qu'il existe un entier naturel $e$ premier à $\ell$ tel que le théorème est vrai pour $\Lambda\left[e^{-1}\right] \epsilon$ $\mathbf{D A}^{\text {ét }}\left(X_{\eta}, \Lambda\left[e^{-1}\right]\right)$. Ceci est clairement suffisant.

Un dévissage standard (utilisant l'hypothèse d'induction) nous ramène au cas où $X$ est intègre et plat sur $S$. D'après [25, Expo. X, Th. 2.4], il existe un carré commutatif

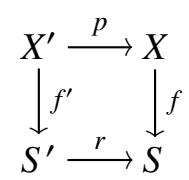

avec $S^{\prime}$ le spectre d'un anneau de valuation discrète hensélien, $X^{\prime}$ un schéma intègre qui est à réduction semi-stable au-dessus de $S^{\prime}$ (au sens affaibli de [5, Déf. 3.3.33]), $r$ un morphisme fini et $p$ une altération de degré générique premier à $\ell$. La condition de semi-stabilité sur $X^{\prime}$ est la suivante. Localement pour la topologie étale, $X^{\prime}$ est isomorphe à $S^{\prime}\left[t_{0}, \cdots, t_{n}\right] /\left(t_{0}^{a_{0}} \cdots t_{r}^{a_{r}}-\pi^{\prime}\right)$ avec $\pi^{\prime}$ une uniformisante de $S^{\prime}$ (cf. [5, Lem. 3.3.37 et 3.3.38]). Dans la suite, on prendra pour $e$ le degré générique de $p$. Quitte à remplacer $\Lambda$ par $\Lambda\left[e^{-1}\right]$, on peut supposer que $e$ est inversible dans $\Lambda$.

Pour démontrer le théorème pour $\Lambda_{X_{\eta}}$, il suffit par l'hypothèse de récurrence de le démontrer pour $u ! \Lambda$ avec $u: U \hookrightarrow X_{\eta}$ l'inclusion d'un ouvert dense au-dessus duquel $p$ est la composition d'un revêtement étale suivi d'un morphisme fini totalement inséparable. Notons $U^{\prime}=p^{-1}(U)$, $u^{\prime}: U^{\prime} \hookrightarrow X_{\eta}^{\prime}$ l'inclusion évidente et $q: U^{\prime} \longrightarrow U$ le morphisme déduit de $p$. D'après [4, Lem. 2.1.165] et puisque $e$ est supposé inversible dans $\Lambda$, le morphisme $\Lambda_{U} \longrightarrow q_{*} \Lambda_{U^{\prime}}$ admet une 
rétraction. Il est alors facile de voir qu'il suffit de montrer le théorème pour $u_{!}^{\prime} \Lambda \in \mathbf{D A}^{\text {ét }}\left(X_{\eta}^{\prime}, \Lambda\right)$. L'hypothèse de récurrence nous ramène à prouver le résultat pour $\Lambda_{X_{\eta}^{\prime}}$.

Le problème étant local pour la topologie étale, il suffit donc de prouver le théorème pour $\Lambda_{X_{\eta}^{\prime}}$ avec $X^{\prime}$ le $S^{\prime}$-schéma donné par $S^{\prime}\left[t_{0}, \cdots, t_{d}\right] /\left(t_{0}^{a_{0}} \cdots t_{d}^{a_{d}}-\pi^{\prime}\right)$. Comme pour le cas d'un $S$ schéma fini, on prendra $L / K$ une sous-extension de $M_{\delta}$ telle que $K^{\prime} \otimes_{K} L$ et $K^{\prime} \otimes_{K} M_{\delta}$ ont les mêmes nombres d'idéaux premiers. (Ici $K^{\prime}$ est le corps des fonctions rationnelles de $S^{\prime}$.) Notons $E$ et $E^{\prime}$ les normalisés des schémas $T \times_{S} S^{\prime}$ et $T^{\prime} \times{ }_{S} S^{\prime}$ respectivement, et $v: E^{\prime} \longrightarrow E$ et $r$ : $E \longrightarrow S^{\prime}$ les morphismes induits. Le résultat découle alors aussitôt de [5, Th. 3.3.4] appliqué aux transformations naturelles $v_{\sigma}^{*} \Psi_{-x_{S^{\prime}} E} r_{\eta}^{*} \longrightarrow \Psi_{-\times_{S^{\prime}} E^{\prime}} v_{\eta}^{*} r_{\eta}^{*}$ vues comme un morphisme de structures de spécialisation au-dessus de $S^{\prime}$. (Pour se débarasser dans [5, Th. 3.3.48] du cas des $b_{n}^{m}$, on utilise que, modulo un revêtement pseudo-galoisien de degré inversible dans $\Lambda$ et localement pour la topologie étale, le morphisme $b_{n}^{m}: S^{\prime}\left[U, U^{-1}\right][T] /\left(T^{n}-U^{m} \cdot \pi^{\prime}\right) \longrightarrow S^{\prime}$ est isomorphe à $b_{n}$ : $\left.S^{\prime}[T] /\left(T^{n}-\pi^{\prime}\right) \longrightarrow S^{\prime}.\right)$

On note $\bar{\sigma}$ le spectre du corps résiduel de $K^{\text {sep }}$ et on pose $X_{\bar{\sigma}}=X \times_{S} \bar{\sigma}$ pour tout $S$-schéma $X$.

Definition 10.14 - On suppose que l'Hypothèse 7.3 est satisfaite. Soient $f: X \longrightarrow S$ un $S$ schéma quasi-projectif. Le foncteur «motif proche total » $\Psi_{f}: \mathbf{D A}^{\mathrm{e} t}\left(X_{\eta}, \Lambda\right) \longrightarrow \mathbf{D A}^{\mathrm{et}}\left(X_{\bar{\sigma}}, \Lambda\right)$ est défini par

$$
\Psi_{f}(A)=\operatorname{hocolim}_{L \subset M_{\delta}}\left(\Psi_{X \times_{S} T}^{\text {mod }}\left(A_{\mid X_{\eta} \otimes_{K} L}\right)\right)_{\mid X_{\bar{\sigma}}}
$$

où $L / K$ parcourt l'ensemble des sous-extensions finies de $M_{\delta} / K$ et $T$ est le spectre de la normalisation de $R$ dans $L$. On obtient ainsi un système de spécialisation pseudo-monö̈dal au-dessus de $(S, \eta, \bar{\sigma})$.

Remarque 10.15 - On laissera au lecteur le soin de donner une formulation précise de la colimite homotopique dans (103) à l'aide du formalisme des dérivateurs algébriques. Dans la suite, nous serons surtout intéressés par le cas où $A$ est constructible. Le Théorème 10.13 affirme alors que le système inductif en question est essentiellement constant; il se calcule donc à l'aide d'une extension finie $L \subset M_{\delta}$ suffisamment grande.

On note $\bar{R}$ la normalisation de $R$ dans $K^{\text {sep }}$. On pose $\bar{\eta}=\operatorname{Spec}\left(K^{\text {sep }}\right), \bar{S}=\operatorname{Spec}(\bar{R})$ et $\bar{\sigma}$ le spectre du corps résiduel de $K^{s e p}$. Étant donné un $S$-schéma $f: X \longrightarrow S$, on forme le diagramme de schémas à carrés cartésiens

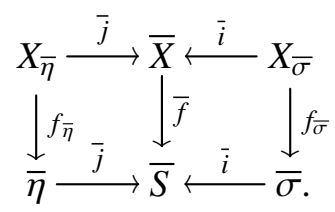

Soit $J \subset \Lambda$ un idéal tel que $\Lambda / J$ est de caractéristique non nulle et inversible sur $S$. On définit alors des foncteurs

$$
\Psi_{f}: \mathbf{D}^{\text {ét }}\left(X_{\eta}, \Lambda / J\right) \longrightarrow \mathbf{D}^{\text {ét }}\left(X_{\bar{\sigma}}, \Lambda / J\right) \quad \text { et } \quad \Psi_{f}: \mathbf{D}^{\text {ét }}\left(X_{\eta}, \Lambda / J^{*}\right) \longrightarrow \mathbf{D}^{\text {ét }}\left(X_{\bar{\sigma}}, \Lambda / J^{*}\right)
$$

par la formule $\Psi_{f}(A)=\bar{i}^{*} \bar{j}_{*} A_{\mid X_{\bar{\eta}}}$. C'est les foncteurs «cycles proches » en cohomologie étale. Il est facile de voir que le second foncteur $\Psi_{f}$ envoie la sous-catégorie $\hat{\mathbf{D}}^{\text {ét }}\left(X_{\eta}, \Lambda_{J}\right)$ dans $\hat{\mathbf{D}}^{\text {ét }}\left(X_{\sigma}, \Lambda_{J}\right)$ induisant ainsi un foncteur « cycles proches »

$$
\Psi_{f}: \hat{\mathbf{D}}^{\text {ét }}\left(X_{\eta}, \Lambda_{J}\right) \longrightarrow \hat{\mathbf{D}}^{\text {ét }}\left(X_{\bar{\sigma}}, \Lambda_{J}\right)
$$

Le résultat suivant est immédiat.

Theoreme 10.16 - On suppose que l'Hypothèse 6.5 est satisfaite. Soit $f: X \longrightarrow S$ un S-schéma quasi-projectif. Il existe alors des faces carrées inversibles (de foncteurs pseudo-monö̈daux)

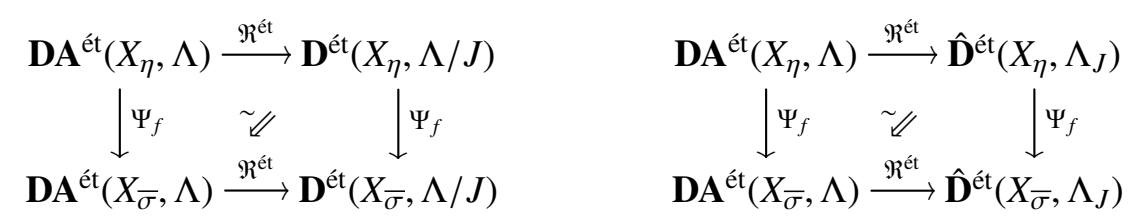


qui sont compatibles aux morphismes de changement de base associés aux $S$-morphismes.

Demonstration Le résultat découle du Théorème 10.11 et de l'isomorphisme évident $\Psi_{f}(A)=$ $\operatorname{colim}_{L \subset M_{\delta}}\left(\Psi_{X \times_{S} T}^{\text {mod }}\left(A_{\mid X_{\eta} \otimes_{K} L}\right)\right)_{\mid X_{\bar{\sigma}}}$ pour $A \in \mathbf{D}^{\text {ét }}\left(X_{\eta}, \Lambda / J\right)$.

En tensorisant par $\mathbb{Q}$ et en passant aux enveloppes pseudo-abléliennes, on obtient également le résultat suivant.

Theoreme 10.17 - On suppose que l'Hypothèse 7.3 est satisfaite. Soit $f: X \longrightarrow S$ un $S$-schéma quasi-projectif. Il existe alors une face carrée inversible (de foncteurs pseudo-monö̈daux)

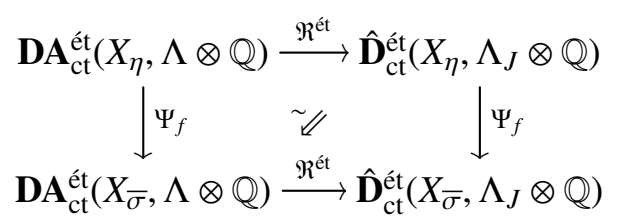

qui est compatible aux morphismes de changement de base associés aux $S$-morphismes.

Une des propriétés bien connues des foncteurs « cycles proches » en cohomologie étale est leur commutation avec le produit tensoriel extérieur. En égale caractéristique nulle, l'analogue motivique de cette propriété est également vrai (cf. [5, Cor. 3.5.18]). On se propose dans le reste de cette section de généraliser cette propriété pour les foncteurs « motif proche total » lorsque la caractéristique résiduelle de $S$ est positive. On commence par le résultat suivant.

Proposition 10.18 - On suppose que l'Hypothèse 7.3 est satisfaite. Soit $F$ un $S$-schéma fini et plat. On note $\bar{F}^{\text {nor }}$ le normalisé de $\bar{F}=F \times_{S} \bar{S}$. Alors, il existe un isomorphisme canonique dans $\mathbf{D A}^{\mathrm{e}}{ }^{\mathrm{t}}\left(F_{\bar{\sigma}}, \Lambda\right)$

$$
\Psi_{F} \Lambda \simeq\left\{\bar{F}^{\text {nor }} \times_{\bar{S}} \bar{\sigma} \rightarrow F_{\bar{\sigma}}\right\}_{*} \Lambda .
$$

De plus, cet isomorphisme est compatible de la manière évidente aux morphismes de $S$-schémas finis et plats.

Demonstration Un dévissage facile nous ramène au cas où $F$ est normal et connexe. Autrement dit, on peut supposer que $F$ est le spectre d'un anneau de valuation discrète fini sur $R$. Soit $L \subset M_{\delta}$ une extension finie de $K$ suffisamment grande de sorte que $\Psi_{F} \Lambda \simeq\left(\Psi_{F \times_{S} T}^{\bmod } \Lambda\right)_{\mid F_{\bar{\sigma}}}$ avec $T$ le spectre du normalisé de $R$ dans $L$. (Une telle extension existe d'après le Théorème 10.13.) Notons $E$ le normalisé de $F \times_{S} T$. Alors, $E=\bigsqcup_{i \in I} E_{i}$ avec $I$ un ensemble fini, $E_{i}=\operatorname{Spec}\left(Q_{i}\right)$ et $Q_{i}$ des anneaux de valuation discrète finis sur $R$. On se donne des uniformisantes $\pi_{i} \in Q_{i}$. On a des identités $\pi=u_{i} \cdot \pi_{i}^{e_{i}}$ avec $u_{i}$ un élément inversible dans $Q_{i}$ et $e_{i} \in \mathbb{N}-\{0\}$. On note $d_{i}$ le plus grand diviseur de $e_{i}$ premier à $p$. Alors, le Théorème 10.6 fournit un isomorphisme

$$
\Psi_{E_{i}}^{\text {mod }} \Lambda \simeq\left\{\left(E_{i}\right)_{\sigma}\left[v_{i}\right] /\left(v_{i}^{d_{i}}-u_{i}\right) \rightarrow\left(E_{i}\right)_{\sigma}\right\}_{*} \Lambda .
$$

On en déduit en fin de compte un isomorphisme canonique

$$
\Psi_{F} \Lambda \simeq\left(\left\{\bigsqcup_{i \in I}\left(E_{i}\right)_{\sigma}\left[v_{i}\right] /\left(v_{i}^{d_{i}}-u_{i}\right) \rightarrow\left(F \times_{S} T\right)_{\sigma}\right\}_{*} \Lambda\right)_{\mid F_{\bar{\sigma}}} .
$$

Pour conclure, il suffit de remarquer que le morphisme $\bar{F}^{\text {nor }} \times_{\bar{S}} \bar{\sigma} \longrightarrow F_{\bar{\sigma}}$ s'identifie au changement de base du morphisme $\bigsqcup_{i \in I}\left(E_{i}\right)_{\sigma}\left[v_{i}\right] /\left(v_{i}^{d_{i}}-u_{i}\right) \longrightarrow\left(F \times \times_{S} T\right)_{\sigma}$ suivant $F_{\bar{\sigma}} \longrightarrow\left(F \times_{S} T\right)_{\sigma}$ lorsque $L \subset M_{\delta}$ est suffisamment grand. La compatibilité avec les morphismes de $S$-schémas finis et plats est laissée au lecteur.

Étant donnés deux $S$-schémas quasi-projectifs $f: X \longrightarrow S$ et $g: Y \longrightarrow S$, on dispose d'un morphisme

$$
\Psi_{f}(A) \otimes \Psi_{g}(B) \longrightarrow \Psi_{f \times_{s} g}(A \otimes B)
$$

binaturel en $A \in \mathbf{D A}^{\text {ét }}\left(X_{\eta}, \Lambda\right)$ et $B \in \mathbf{D A}^{\text {ét }}\left(Y_{\eta}, \Lambda\right)$. Il est donné par la composition de

$$
p r_{1}^{*} \Psi_{f}(A) \otimes p r_{2}^{*} \Psi_{g}(B) \longrightarrow \Psi_{f \times_{S} g}\left(p r_{1}^{*}(A)\right) \otimes \Psi_{f \times_{S} g}\left(p r_{2}^{*}(B)\right) \longrightarrow \Psi_{f \times_{S} g}\left(p r_{1}^{*}(A) \otimes p r_{2}^{*}(B)\right)
$$

où $p r_{1}$ et $p r_{2}$ désigent la projection sur le premier et le second facteur d'un porduit fibré. On a le résultat suivant. 
Theoreme 10.19 - On suppose que l'Hypothèse 7.3 est satisfaite. Alors le morphisme (104) est un isomorphisme. (En particulier, $\Psi_{S}: \mathbf{D A}^{\mathrm{e} t}(\eta, \Lambda) \longrightarrow \mathbf{D A}^{\mathrm{e}}(\bar{\sigma}, \Lambda)$ est monö̈dal, symétrique et unitaire.)

Demonstration On suit la preuve de [5, Th. 3.5.17]. On fixe d'abord le $S$-schéma $X$ et l'objet $A$. On obtient alors un morphisme de systèmes de spécialisation

$$
\Psi_{f}(A) \otimes \Psi_{-}(-) \longrightarrow \Psi_{f \times_{S}-}(A \otimes-) .
$$

On cherche à montrer que ce morphisme de systèmes de spécialisation est inversible. Un dévissage similaire à celui utilisé au début de la preuve du Théorème 10.13 nous ramène au cas où $B=\Lambda$. (On utilise ici que les foncteurs $\Psi(-)$ et $-\otimes-$ commutent aux sommes infinies.)

Ensuite, on affirme qu'il suffit de traiter le cas où $g$ est fini et plat. (On suppose toujours que $B=\Lambda$.) En effet, supposons que (104) est inversible lorsque $g$ est fini et plat (et $B=\Lambda$ ). On raisonne alors par récurrence sur la dimension de $Y_{\eta}$ comme dans la preuve du Théorème 10.13. Les grandes lignes du raisonnement sont les suivantes. Un dévissage standard permet de supposer que $Y$ est intègre et plat sur $S$. On applique ensuite [25, Expo. X, Th. 2.4] et un dévissage pour se ramener au cas où $Y$ est à réduction semi-stable (au sens affaibli de [5, Déf. 3.3.33]) au-dessus de $S^{\prime}=\operatorname{Spec}\left(R^{\prime}\right)$ avec $R^{\prime}$ un anneau de valuation discrète fini sur $R$. Le résultat recherché découle alors de [5, Th. 3.3.4] appliqué au morphisme de systèmes de spécialisation (105) restreint à $S^{\prime}$.

Par symétrie, on peut aussi supposer que $f$ est fini et plat, et que $A=\Lambda$. Le résultat recherché découle alors de la Proposition 10.18. En effet, si $F$ et $G$ sont deux $S$-schémas finis et plats, et $H=F \times_{S} G$, on a un morphisme canonique $\bar{H}^{\text {nor }} \longrightarrow \bar{F}^{\text {nor }} \times{ }_{\bar{S}} \bar{G}^{\text {nor }}$ qui est un homéomorphisme universel. En particulier, $\bar{H}^{n o r} \times \bar{S}_{\bar{S}} \longrightarrow\left(\bar{F}^{n o r} \times \bar{S}^{n o r}\right) \times \bar{S}_{\bar{\sigma}}$ induit un isomorphisme sur les schémas réduits associés et le morphisme

$$
\left(\left\{\bar{F}^{\text {nor }} \times_{\bar{S}} \bar{\sigma} \rightarrow F_{\bar{\sigma}}\right\}_{*} \Lambda\right) \otimes\left(\left\{\bar{G}^{\text {nor }} \times_{\bar{S}} \bar{\sigma} \rightarrow G_{\bar{\sigma}}\right\}_{*} \Lambda\right) \longrightarrow\left\{\bar{H}^{\text {nor }} \times_{\bar{S}} \bar{\sigma} \rightarrow H_{\bar{\sigma}}\right\}_{*} \Lambda
$$

est alors un isomorphisme. La compatibilité de l'isomorphisme de la Proposition 10.18 avec les morphismes de $S$-schémas finis et plats permet maintenant de conclure.

Theoreme 10.20 - On suppose que l'Hypothèse 7.3 est satisfaite. Soit $f: X \longrightarrow S$ un $S$-schéma quasi-projectif. On définit des opérateurs de dualité sur $\mathbf{D A}^{\mathrm{e}}\left(X_{\eta}, \Lambda\right), \mathbf{D A}^{\text {ét }}\left(X_{\sigma}, \Lambda\right)$ et $\mathbf{D} \mathbf{A}^{\text {ét }}\left(X_{\bar{\sigma}}, \Lambda\right)$ $\operatorname{par} \mathrm{D}_{\eta}(-)=\underline{\operatorname{Hom}}\left(-, f_{\eta}^{!} \Lambda\right), \mathrm{D}_{\sigma}(-)=\underline{\operatorname{Hom}}\left(-, f_{\bar{\sigma}}^{!} \Lambda\right)$ et $\mathrm{D}_{\bar{\sigma}}(-)=\underline{\operatorname{Hom}}\left(-, f_{\bar{\sigma}}^{!} \Lambda\right)$. On a alors des morphismes canoniques

$$
\Psi_{f}^{\text {mod }} \mathrm{D}_{\eta}(A) \longrightarrow \mathrm{D}_{\sigma} \Psi_{f}^{\text {mod }}(A) \quad \text { et } \quad \Psi_{f} \mathrm{D}_{\eta}(A) \longrightarrow \mathrm{D}_{\bar{\sigma}} \Psi_{f}(A)
$$

qui sont inversibles lorsque $A \in \mathbf{D A}^{\text {ét }}\left(X_{\eta}, \Lambda\right)$ est constructible.

Demonstration Pour les foncteurs «motif proche total », le morphisme en question est obtenu par adjonction à partir de la composition de

$$
\Psi_{f} \underline{\operatorname{Hom}}\left(A, f_{\eta}^{!} \Lambda\right) \otimes \Psi_{f}(A) \longrightarrow \Psi_{f}\left(\underline{\operatorname{Hom}}\left(A, f_{\eta}^{!} \Lambda\right) \otimes A\right) \longrightarrow \Psi_{f} f_{\eta}^{!} \Lambda \longrightarrow f_{\bar{\sigma}}^{!} \Psi_{S} \Lambda \simeq f_{\bar{\sigma}}^{!} \Lambda .
$$

Pour les foncteurs «motif proche modéré », c'est donné par adjonction à partir d'une composition similaire où $\Psi_{f}$ est remplacé par $\Psi_{f}^{\text {mod }}$ et $f_{\bar{\sigma}}$ est remplacé par $f_{\sigma}$. Dans le suite, on suppose que $A$ est constructible et on cherche à montrer que les morphismes (106) sont inversibles.

Soit $L \subset M_{\delta}$ une extension finie de $K$ suffisamment grande. On note $T$ le spectre du normalisé de $R$ dans $L$ et $t: T \longrightarrow S$ le morphisme évident ainsi que son changement de base $t: X \times_{S} T \longrightarrow X$. D'après le Théorème 10.13, on peut supposer que

$$
\Psi_{f} A \simeq\left(\Psi_{X \times_{S} T}^{\bmod } t_{\eta}^{*} A\right)_{\mid X_{\bar{\sigma}}} \quad \text { et } \quad \Psi_{f} \mathrm{D}_{\eta}(A) \simeq\left(\Psi_{X \times_{S} T}^{\bmod } t_{\eta}^{*} \mathrm{D}_{\eta}(A)\right)_{\mid X_{\bar{\sigma}}} \simeq\left(\Psi_{X \times_{S} T}^{\bmod } \mathrm{D}_{\eta}\left(t_{\eta}^{*} A\right)\right)_{\mid X_{\bar{\sigma}}} .
$$

(L'isomorphisme de commutation $t_{\eta}^{*} \mathrm{D}_{\eta} \simeq \mathrm{D}_{\eta} t_{\eta}^{*}$ est justifié par le fait que $t_{\eta}$ est un morphisme étale.) L'accouplement composé (107) s'identifie alors à l'image inverse suivant le morphisme $X_{\bar{\sigma}} \longrightarrow\left(X \times_{S} T\right)_{\sigma}$ de l'accouplement composé

$$
\Psi_{X \times_{S} T}^{\bmod } \mathrm{D}_{\eta}\left(t_{\eta}^{*} A\right) \otimes \Psi_{X \times_{S} T}^{\bmod }\left(t_{\eta}^{*} A\right) \longrightarrow\left(f \times_{S} T\right)_{\sigma}^{!} t_{\sigma}^{!} \Lambda \simeq\left(f \times_{S} T\right)_{\sigma}^{!} t_{\sigma}^{*} \Lambda .
$$


(On utilise ici que $\left(\left(f \times_{S} T\right)_{\sigma}^{!} t_{\sigma}^{*} \Lambda\right)_{\mid X_{\bar{\sigma}}}$ s'identifie canoniquement à $f_{\bar{\sigma}}^{!} \Lambda$ étant donné que le morphisme $T_{\bar{\sigma}} \longrightarrow\left(X \times_{S} T\right)_{\sigma}$ est le composé d'un morphisme pro-étale suivie d'un morphisme entier, surjectif et totalement inséparable.) On en déduit aussitôt que le morphisme $\Psi_{f} \mathrm{D}_{\eta}(A) \longrightarrow \mathrm{D}_{\bar{\sigma}} \Psi_{f}(A)$ s'identifie à un changement de base d'un morphisme du type $\Psi_{f}^{\bmod } \mathrm{D}_{\eta}(A) \longrightarrow \mathrm{D}_{\sigma} \Psi_{f}^{\bmod }(A)$. Ainsi, il est suffisant de traiter le cas des foncteurs « motif proche modéré ».

On suit la preuve de [5, Th. 3.5.20]. Ainsi, on considère le morphisme de systèmes de spécialisation

$$
\Psi^{\text {mod }} \circ \mathrm{D}_{\eta} \longrightarrow \mathrm{D}_{\sigma} \circ \Psi^{\bmod }
$$

et on cherche à montrer que c'est un isomorphisme après restriction à $\mathbf{D} A_{\mathrm{ct}}^{\text {et }}(-, \Lambda)$. Un dévissage similaire à celui utilisé au début de la preuve du Théorème 10.13 nous ramène au cas où $A=\Lambda$. Il s'agit alors de montrer que l'accouplement composé

$$
\left(\Psi_{f}^{\text {mod }} f_{\eta}^{!} \Lambda\right) \otimes\left(\Psi_{f}^{\text {mod }} \Lambda\right) \longrightarrow \Psi_{f}^{\text {mod }} f_{\eta}^{!} \Lambda \longrightarrow f_{\sigma}^{!} \Psi_{S}^{\text {mod }} \Lambda \simeq f_{\sigma}^{!} \Lambda
$$

est un accouplement parfait, i.e., induit un isomorphisme $\Psi_{f}^{\bmod } f_{\eta}^{!} \Lambda \simeq \underline{\operatorname{Hom}}\left(\Psi_{f}^{\bmod } \Lambda, f_{\sigma}^{!} \Lambda\right)$.

On raisonne par récurrence sur la dimension de Krull de $X_{\eta}$. Lorsque cette dimension est nulle, on peut supposer que $X=\operatorname{Spec}(Q)$ avec $Q$ un anneau de valuation discrète fini sur $S$. Dans ce cas, $f_{\eta}^{!} \Lambda=\Lambda$ et $f_{\bar{\sigma}}^{!} \Lambda=\Lambda$. Soit $\varpi \in Q$ une uniformisante et supposons que $\pi=u \cdot \varpi^{e}$ avec $u \in Q$ inversible et $e \in \mathbb{N}-\{0\}$. Comme dans la preuve du Théorème 10.6 et celle de [5, Th. 3.5.20], on peut remplacer $X$ par le normalisé de $X \times_{S} S\left[\pi^{1 / n}\right]$ pour tout $n \in \mathbb{N}^{\prime \times}$. Ceci nous ramène au cas où $e$ est une puissance de $p$. Dans ce cas, le Théorème 10.7 fournit des isomorphismes canoniques $\Psi_{f}^{\text {mod }} \Lambda \simeq \Lambda$ et l'accouplement (109) s'identifie à l'isomorphisme canonique $\Lambda \otimes \Lambda \simeq \Lambda$. Ceci permet de conclure.

On passe maintenant au cas $\operatorname{krdim}\left(X_{\eta}\right) \geq 1$. On raisonne comme dans la preuve du Théorème 10.13. Les grandes lignes du raisonnement sont les suivantes. Un dévissage standard permet de supposer que $X$ est intègre et plat sur $S$. On applique ensuite [25, Expo. X, Th. 2.4] et un dévissage pour se ramener au cas où $X$ est à réduction semi-stable (au sens affaibli de [5, Déf. 3.3.33]) audessus de $S^{\prime}=\operatorname{Spec}\left(R^{\prime}\right)$ avec $R^{\prime}$ un anneau de valuation discrète fini sur $R$. Le résultat recherché découle alors de [5, Th. 3.3.4] appliqué au morphisme de systèmes de spécialisation (108) restreint à $S^{\prime}$. Les détails sont laissés au lecteur.

Remarque 10.21 — L'analogue du Théorème 10.20 est également vrai pour le système de spécialisation $\Upsilon$. Le lecteur vérifiera facilement que la preuve de [5, Th. 3.4.20] s'adapte au cas où la caractéristique résiduelle de $S$ est positive.

\section{Comparaison de monodromie}

Dans cette section on étend l'opérateur de monodromie pour les foncteurs «partie unipotente du motif proche » construits dans $[\mathbf{5}, \S 3.6]$ du cas où $S$ est d'égale caractéristique nulle au cas où la caractéristique résiduelle de $S$ est quelconque. Ensuite, on explique le lien entre cet opérateur de monodromie et son analogue en cohomologie étale. Au passage, nous corrigeons une erreur dans [5, Th. 3.6.10] liée à la construction de l'opérateur de monodromie motivique.

Comme dans [5, §3.6], l'opérateur de monodromie sera d'abord construit sur le système de spécialisation logarithmique. Ce système a été construit dans $[\mathbf{5}, \S 3.6 .3]$ pour $S$ de caractéristiques résiduelles nulles. Notre première tâche sera donc de s'assurer que cette construction s'étend sans problème au-dessus d'une base plus générale. Nous aurons d'abord besoin de calculer certains groupes de cohomologie motivique. Nous donnons un énoncé plus précis que nécessaire.

Proposition 11.1 - Soient $S$ un schéma, $\Lambda$ un anneau de coefficients et $M$ un $\Lambda$-module. On pose $M_{S}=\operatorname{Sus}_{T}^{0}\left(M_{c s t}\right) \in \mathbf{D A}^{\mathrm{e}}(S, \Lambda)$ avec $M_{c s t}$ le préfaisceau constant de valeur $M$ sur $\mathrm{Sm} / S$.

(a) On suppose que l'Hypothèse 7.3 est satisfaite. Alors, on a :

$$
\operatorname{hom}_{\mathbf{D A}^{\mathrm{et}}(S, \Lambda)}\left(\Lambda_{S}, M_{S}(m)[n]\right)=\left\{\begin{array}{clc}
M^{\pi_{0}(S)} & \text { si } \quad m=n=0 \\
0 & \text { si } & m \leq 0 \text { et } n<0 .
\end{array}\right.
$$


(Ci-dessus, $\pi_{0}(S)$ est l'ensemble des composantes connexes de $S$.)

(b) On suppose que $\Lambda$ est une $\mathbb{Q}$-algèbre. Alors, on a:

$$
\operatorname{hom}_{\mathbf{D A}^{\mathrm{et}}(S, \Lambda)}\left(\Lambda_{S}, M_{S}(m)[n]\right)=0 \text { si } m<0 .
$$

Demonstration On divise la preuve en plusieurs étapes.

Étape $A$ : Le but de cette étape est de se ramener au cas $\Lambda=\mathbb{Q}$ et $M=\mathbb{Q}$. On se concentre sur la partie (a) de l'énoncé ; pour la partie (b) ceci est plus facile.

Supposons d'abord que $M$ est de torsion en tant que groupe abélien. Alors, $M$ est une colimite filtrante, suivant $N \in \mathbb{N}^{\times}$, de ses sous-modules formés des éléments annulés par $N$. Étant donné que $\Lambda_{S}(0) \in \mathbf{D A}^{\text {ét }}(S, \Lambda)$ est compact, on se ramène aussitôt à traiter le cas où $M$ est lui-même annulé par un certain $N \in \mathbb{N}-\{0\}$. Dans ce cas, $M$ est un $\Lambda / N \Lambda$-module et on a un isomorphisme d'adjonction

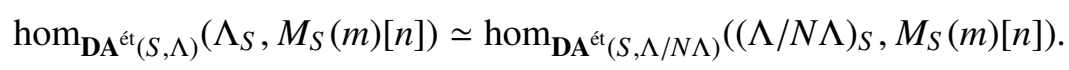

Par ailleurs, le Théorème 4.1 fournit une équivalence $\mathbf{D}^{\text {ét }}(S, \Lambda / N \Lambda) \simeq \mathbf{D A}^{\text {ét }}(S, \Lambda / N \Lambda)$. On est donc ramené à prouver le résultat analogue en cohomologie étale, ce qui est une trivialité.

Reprenons le cas général. On a une suite exacte courte de $\Lambda$-modules

$$
0 \longrightarrow M_{\text {tor }} \longrightarrow M \longrightarrow M^{\prime} \longrightarrow 0
$$

avec $M_{\text {tor }} \subset M$ le plus grand sous-module de $M$ qui soit de torsion en tant que groupe abélien. Cette suite exacte induit une suite exacte longue après application de $\operatorname{hom}_{\mathbf{D A}^{\mathrm{tt}}(S, \Lambda)}\left(\Lambda_{S},(-)_{S}(m)\right)$. Vu que le cas de $M_{t o r}$ est connu, on se ramène à traiter le cas de $M^{\prime}$. Autrement dit, on peut supposer que $M$ est sans torsion (en tant que groupe abélien). Ceci permet de considérer une autre suite exacte courte de $\Lambda$-modules

$$
0 \longrightarrow M \longrightarrow M \otimes \mathbb{Q} \longrightarrow M \otimes(\mathbb{Q} / \mathbb{Z}) \longrightarrow 0
$$

Comme avant, le cas de $M \otimes(\mathbb{Q} / \mathbb{Z})$ est connu. La suite exacte longue obtenue en appliquant $\operatorname{hom}_{\mathbf{D A}^{\text {et }}(S, \Lambda)}\left(\Lambda_{S},(-)_{S}(m)\right)$ nous ramène à traiter le cas de $M \otimes \mathbb{Q}$. Autrement dit, on peut supposer que $M$ est uniquement divisible (en tant que groupe abélien). Dans ce cas, on a des isomorphismes d'adjonction

$$
\begin{aligned}
\operatorname{hom}_{\mathbf{D A}^{\mathrm{et}}(S, \Lambda)}\left(\Lambda_{S}, M_{S}(m)[n]\right) & \simeq \operatorname{hom}_{\mathbf{D A}^{\mathrm{et}}(S, \Lambda \otimes \mathbb{Q})}\left((\Lambda \otimes \mathbb{Q})_{S}, M_{S}(m)[n]\right) \\
& \simeq \operatorname{hom}_{\mathbf{D A}^{\mathrm{et}}(S, \mathbb{Q})}\left(\mathbb{Q}_{S}, M_{S}(m)[n]\right) .
\end{aligned}
$$

Ainsi, on peut supposer que $\Lambda=\mathbb{Q}$. Mais alors, $M$ est une somme directe de copies de $\mathbb{Q}$ et on peut aussi supposer que $M=\mathbb{Q}$.

Étape B : Dans la suite, on pose $\mathrm{H}_{\mathcal{M}}^{n, m}(S, \mathbb{Q})=\operatorname{hom}_{\mathbf{D A}^{\mathrm{et}}(S, \mathbb{Q})}\left(\mathbb{Q}_{S}, \mathbb{Q}_{S}(m)[n]\right)$. Les propriétés à démontrer sont locales pour la toplogie de Nisnevich. En effet, étant donné un carré Nisnevich

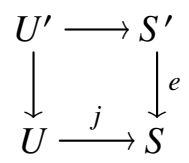

(i.e., un carré cartésien avec $j$ une immersion ouverte et $e$ un morphisme étale induisant un isomorphisme $S^{\prime}-U^{\prime} \simeq S-U$ ), on a une suite exacte longue de Mayer-Vietoris

$$
\cdots \longrightarrow \mathrm{H}_{\mathcal{M}}^{n, m}(S, \mathbb{Q}) \longrightarrow \mathrm{H}_{\mathcal{M}}^{n, m}\left(S^{\prime}, \mathbb{Q}\right) \bigoplus \mathrm{H}_{\mathcal{M}}^{n, m}(U, \mathbb{Q}) \longrightarrow \mathrm{H}_{\mathcal{M}}^{n, m}\left(U^{\prime}, \mathbb{Q}\right) \longrightarrow \cdots
$$

Il en découle aussitôt que si l'énoncé est vrai pour $S^{\prime}, U$ et $U^{\prime}$, il l'est aussi pour $S$. En particulier, on peut supposer que $S=\operatorname{Spec}(\mathcal{A})$ est affine. Or, d'après la Proposition 3.20, on a un isomorphisme

$$
\mathrm{H}_{\mathcal{M}}^{n, m}(\operatorname{Spec}(\mathcal{A}), \mathbb{Q}) \simeq \operatorname{colim}_{A \subset \mathcal{A}} \mathrm{H}_{\mathcal{M}}^{n, m}(\operatorname{Spec}(A), \mathbb{Q})
$$

où $A$ parcourt l'ensemble filtrant des sous-algèbres de $\mathcal{A}$ de type fini sur $\mathbb{Z}\left[1 / p, p \in \mathcal{P}_{\mathcal{A}}\right]$ avec $\mathcal{P}_{\mathcal{A}}$ l'ensemble des nombres premiers inversibles dans $\mathcal{A}$. Il est donc suffisant de considérer le cas où $S$ est un $D$-schéma de type fini avec $D$ le spectre d'un anneau de Dedekind contenu dans $\mathbb{Q}$. 
On raisonne maintenant par induction sur la dimension de $\operatorname{Krull} \operatorname{de} S$. Lorsque $\operatorname{krdim}(S)=0$, on peut supposer que $S=\operatorname{Spec}(k)$ avec $k$ un corps parfait (en fait, une extension finie de $\mathbb{Q}$ ou un corps fini). Or, d'après le Théorème B.1, on a une équivalence de catégories $\mathbf{D A}^{\text {ét }}(k, \mathbb{Q}) \simeq \mathbf{D M}^{\text {ét }}(k, \mathbb{Q})$. Vu le « cancellation theorem » de Voevodsky [51], il suffit donc de montrer que

$$
\operatorname{hom}_{\mathbf{D M}} \mathbf{e f f}_{(k, \mathbb{Q})}\left(\mathbb{Q}_{t r}\left(\left(\mathbb{G m}_{k}, 1\right)^{\wedge r}\right), \mathbb{Q}_{t r}(\operatorname{Spec}(k))[n]\right)=\left\{\begin{array}{ccc}
\mathbb{Q} & \text { si } & r=n=0, \\
0 & \text { si } & r=0 \text { et } n \neq 0, \\
0 & \text { si } & r>0 .
\end{array}\right.
$$

Or, le faisceau avec transferts constant $\mathbb{Q}=\mathbb{Q}_{t r}(\operatorname{Spec}(k))$ est invariant par homotopie. Il est donc $\left(\mathbb{A}^{1}\right.$, ét)-local. Les groupes de morphismes en question se calculent donc dans la catégorie dérivée des faisceaux étales. Le résultat recherché s'ensuit.

On suppose à présent que $\operatorname{krdim}(S) \geq 1$. Appelons $S^{\prime}$ le normalisé de $S$, et fixons un fermé $Z \subset S$ partout de codimension non nulle et tel que $(S-Z)_{\text {red }}$ est normal. On pose $Z^{\prime}=S^{\prime} \times{ }_{S} Z$. Le morphisme $\left(S^{\prime}-Z^{\prime}\right) \longrightarrow(S-Z)_{\text {red }}$ est alors un isomorphisme. L'axiome de localité et le théorème de changement de base pour un morphisme fini fournissent un triangle distingué

$$
\mathbb{Q}_{S} \longrightarrow\left\{S^{\prime} \rightarrow S\right\}_{*} \mathbb{Q}_{S^{\prime}} \bigoplus\{Z \longrightarrow S\}_{*} \mathbb{Q}_{Z} \longrightarrow\left\{Z^{\prime} \rightarrow S\right\}_{*} \mathbb{Q}_{Z^{\prime}} \longrightarrow
$$

qui induit une suite exacte longue

$$
\cdots \longrightarrow \mathrm{H}_{\mathcal{M}}^{n, m}(S, \mathbb{Q}) \longrightarrow \mathrm{H}_{\mathcal{M}}^{n, m}\left(S^{\prime}, \mathbb{Q}\right) \bigoplus \mathrm{H}_{\mathcal{M}}^{n, m}(Z, \mathbb{Q}) \longrightarrow \mathrm{H}_{\mathcal{M}}^{n, m}\left(Z^{\prime}, \mathbb{Q}\right) \longrightarrow \cdots
$$

D'après l'hypothèse de récurrence sur la dimension de Krull, il suffit de traiter le cas de $S^{\prime}$. Autrement dit, on peut supposer que $S$ est normal. C'est ce qu'on fera dans la suite.

Étape $C$ : Dans cette étape, on traite le cas où $S$ est de caractéristique positive. On ne restreint pas la généralité en supposant que $S$ est connexe. Puisque $S$ est normal, c'est donc un schéma de type fini sur $\mathbb{F}_{p}$ pour un certain nombre premier $p$ non inversible sur $D$.

D'après de Jong [29], il existe un morphisme propre et surjectif $f: T \longrightarrow S$ tel que $T$ est intègre et régulier, et $e$ est génériquement un revêtement pseudo-galoisien de groupe de Galois $G$. On peut donc trouver $Z \subset S$ un fermé de codimension non nulle tel que $S-Z$ est normal et $f: T-f^{-1}(Z) \longrightarrow S-Z$ est la composition d'un revêtement étale galoisien et d'un morphisme fini, surjectif, totalement inséparable. Notons $S^{\prime}$ le normalisé de $S$ dans $T-f^{-1}(Z)$. C'est un $S$-schéma fini muni d'une action de $G$ et $S^{\prime} / G \longrightarrow S$ est totalement inséparable puisque $S$ est normal. D'après [4, Lem. 2.1.165], on a un isomorphisme canonique $\Lambda_{S} \longrightarrow\left(\left\{S^{\prime} \rightarrow S\right\}_{*} \Lambda_{S^{\prime}}\right)^{G}$. Il s'ensuit que $\mathrm{H}_{\mathcal{M}}^{n, m}(S, \mathbb{Q}) \longrightarrow \mathrm{H}_{\mathcal{M}}^{n, m}\left(S^{\prime}, \mathbb{Q}\right)^{G}$. Il est donc suffisant de considérer le cas de $S^{\prime}$.

Quitte à remplacer $S$ par $S^{\prime}$, on peut donc supposer que $f: T \longrightarrow S$ est un isomorphisme audessus de $S-Z$. L'axiome de localité et le théorème de changement de base pour un morphisme projectif fournissent un triangle distingué

$$
\mathbb{Q}_{S} \longrightarrow\{T \rightarrow S\}_{*} \mathbb{Q}_{T} \bigoplus\{Z \longrightarrow S\}_{*} \mathbb{Q}_{Z} \longrightarrow\left\{f^{-1}(Z) \rightarrow S\right\}_{*} \mathbb{Q}_{f^{-1}(Z)} \longrightarrow
$$

qui induit une suite exacte longue

$$
\cdots \longrightarrow \mathrm{H}_{\mathcal{M}}^{n, m}(S, \mathbb{Q}) \longrightarrow \mathrm{H}_{\mathcal{M}}^{n, m}(T, \mathbb{Q}) \bigoplus \mathrm{H}_{\mathcal{M}}^{n, m}(Z, \mathbb{Q}) \longrightarrow \mathrm{H}_{\mathcal{M}}^{n, m}\left(f^{-1}(Z), \mathbb{Q}\right) \longrightarrow \cdots
$$

D'après l'hypothèse de récurrence, il est suffisant de traiter le cas de $T$. Autrement dit, on peut supposer que $S$ est lisse sur un corps parfait $k$.

En utilisant le Théorème B.1 et le « cancellation theorem » de Voevodsky [51], on se ramène à montrer que

$$
\operatorname{hom}_{\mathbf{D M}^{\mathrm{eff}}(k, \mathbb{Q})}\left(\mathbb{Q}_{t r}\left(S \times_{k}\left(\mathbb{G}_{k}, 1\right)^{\wedge r}\right), \mathbb{Q}_{t r}(0)[n]\right)=\left\{\begin{array}{ccc}
\mathbb{Q}^{\pi_{0}(S)} & \text { si } & r=n=0, \\
0 & \text { si } & r=0 \text { et } n \neq 0, \\
0 & \text { si } & r>0 .
\end{array}\right.
$$

Or, le faisceau avec transferts constant $\mathbb{Q}=\mathbb{Q}_{t r}(\operatorname{Spec}(k))$ est invariant par homotopie. Il est donc $\left(\mathbb{A}^{1}\right.$, ét)-local. Les groupes de morphismes en question se calculent donc dans la catégorie dérivée des faisceaux étales. Le résultat recherché s'ensuit. 
JOSEPH AYOUB

Étape $D$ : On suppose ici que $S \longrightarrow D$ est dominant avec $S$ connexe et normal. La résolution des singularités de Hironaka [19] appliquée à $S \otimes \mathbb{Q}=S \times{ }_{D} \operatorname{Spec}(\mathbb{Q})$ fournit un morphisme propre et surjectif $f: T \longrightarrow S$ tel que $T \otimes \mathbb{Q}$ est lisse et $f$ est un isomorphisme au-dessus d'un ouvert dense de $S$. On peut aussi supposer que $T$ est normal et connexe. En reprenant l'argument de l'Étape C, et notamment l'analogue de la suite exacte longue (111), on voit qu'il suffit de traiter le cas de $T$. Autrement dit, on peut supposer que $S \otimes \mathbb{Q}$ est lisse. Il existe alors un ouvert dense $D_{0} \subset D$ tel que $S_{0}=S \times_{D} D_{0}$ est lisse sur $D_{0}$. Pour $x \in D-D_{0}$, notons $D_{x}^{h}$ l'hensélisé de $D$ en $x$ et $S_{x}^{h}=S \times_{D} D_{x}^{h}$. En passant à la colimite dans (110) suivant les voisinages étales de $D-D_{0}$ dans $D$, on obtient une suite exacte longue de Mayer-Vietoris

$$
\longrightarrow \mathrm{H}_{\mathcal{M}}^{n, m}(S, \mathbb{Q}) \longrightarrow\left(\bigoplus_{x \in D-D_{0}} \mathrm{H}_{\mathcal{M}}^{n, m}\left(S_{x}^{h}, \mathbb{Q}\right)\right) \bigoplus \mathrm{H}_{\mathcal{M}}^{n, m}\left(S_{0}, \mathbb{Q}\right) \longrightarrow \bigoplus_{x \in D-D_{0}} \mathrm{H}_{\mathcal{M}}^{n, m}\left(S_{x}^{h} \otimes \mathbb{Q}, \mathbb{Q}\right) \longrightarrow
$$

Il est donc suffisant de traiter le deux cas suivants :

$-S$ est lisse sur $D$,

- $D$ est le spectre d'un corps de caractéristique nulle ou d'un anneau de valuation discrète hensélien.

Le second cas est traité en suivant la méthode de l'Étape C qui est basée sur la technique de désingularisation de de Jong [29]. On laisse les détails aux lecteurs.

Dans le reste de la preuve, on explique comment traiter le cas où $S$ est lisse sur $D$. L'ingrédient clef est la pureté absolue (cf. le Corollaire 7.5). Soit $p$ un nombre premier non inversible sur $D$ et notons $i_{p}: \operatorname{Spec}\left(\mathbb{F}_{p}\right) \hookrightarrow D$ l'inclusion canonique. Notons aussi $j_{\mathbb{Q}}: \operatorname{Spec}(\mathbb{Q}) \hookrightarrow S$ l'inclusion du point générique. On a alors un triangle distingué dans $\mathbf{D A}^{\text {ét }}(S, \Lambda)$ :

$$
\bigoplus_{p} i_{p}^{!} \Lambda_{S} \longrightarrow \Lambda_{S} \longrightarrow\left(j_{\mathbb{Q}}\right)_{*} \Lambda_{S \otimes \mathbb{Q}} \longrightarrow
$$

où $p$ parcourt l'ensemble des nombres premiers non inversibles sur $D$. Ce triangle est obtenu en prenant une colimite, suivant les ouverts non vides de $D$, de triangles distingués de localité (cf. [4, Prop. 1.4.9]). Or, d'après le Corollaire 7.5, on a un isomorphisme $i_{p}^{!} \Lambda_{S} \simeq \Lambda_{S_{p}}(-1)[-2]$ avec $S_{p}=S \times_{D} \operatorname{Spec}\left(\mathbb{F}_{p}\right)$. Il s'ensuit une suite exacte longue

$$
\cdots \rightarrow \bigoplus_{p} \mathrm{H}_{\mathcal{M}}^{m-1, n-2}\left(S_{p}, \mathbb{Q}\right) \longrightarrow \mathrm{H}_{\mathcal{M}}^{m, n}(S, \mathbb{Q}) \longrightarrow \mathrm{H}_{\mathcal{M}}^{m, n}(S \otimes \mathbb{Q}, \mathbb{Q}) \longrightarrow \cdots .
$$

D'après l'hypothèse de récurrence sur la dimension de Krull appliquée aux $S_{p}$, il suffit de traiter le cas du schéma $S \otimes \mathbb{Q}$. Autrement dit, on peut supposer que $D=\operatorname{Spec}(\mathbb{Q})$. Ce cas est traité comme dans l'Étape C. Ceci termine la preuve de la proposition.

Remarque 11.2 - On peut utiliser la Proposition 11.1 pour démontrer le fait suivant. Soient $S$ un schéma intègre et $L \in \mathbf{D A}^{\text {ét }}(S, \Lambda)$. On suppose que localement pour la topologie de Zariski, $L$ est isomorphe à $\Lambda_{S}(n)$ avec $n \in \mathbb{Z}$ fixé. Alors, il existe un isomorphisme global $L \simeq \Lambda_{S}(n)$ dans $\mathbf{D A}^{\text {ét }}(S, \Lambda)$. En effet, soit $\left(U_{i}\right)_{1 \leq i \leq r}$ un recouvrement Zariski et supposons donnés des isomorphismes $a_{i}: L_{\mid U_{i}} \simeq \Lambda_{U_{i}}(n)$. Nous allons construire un isomorphisme $L \simeq \Lambda_{S}(n)$ en raisonnant pas récurrence sur $r$. Lorsque $r=1$, il n'y a rien à faire. On suppose donc que $r \geq 2$ et on pose $V=\bigcup_{i=1}^{r-1} U_{i}$. L'hypothèse de récurrence fournit un isomorphisme $b: L_{\mid V} \simeq \Lambda_{V}(n)$. La composition de

$$
\Lambda_{V \cap U_{r}}(n) \stackrel{b^{-1}}{\sim} L_{\mid V \cap U_{r}} \stackrel{a_{r}}{\sim} \Lambda_{V \cap U_{r}}(n)
$$

définit un élément inversible de $\Lambda^{\pi_{0}\left(V \cap U_{r}\right)} \simeq \operatorname{hom}_{\mathbf{D A}^{\text {ét }}\left(V \cap U_{r}\right)}(\Lambda(n), \Lambda(n))$. (On utilise ici la Proposition 11.1.) Puisque $S$ est intègre, $\pi_{0}\left(V \cap U_{r}\right)$ est un singleton. Ainsi, quitte à multiplier l'isomorphisme $a_{r}$ par un scalaire inversible, on peut supposer que $b$ et $a_{r}$ coïncident sur $V \cap U_{r}$. Le carré 
solide dans le diagramme

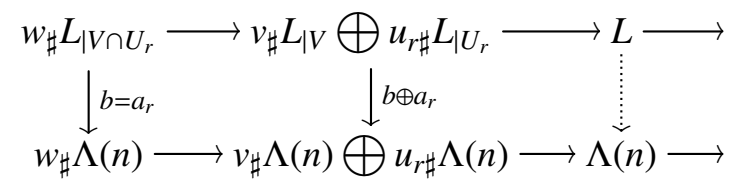

est donc commutatif. (Ci-dessus, $w, v$ et $u_{r}$ désignent les inclusions de $V \cap U_{r}, V$ et $U_{r}$ dans $S$.) On peut donc compléter le diagramme ci-dessus en un morphisme de triangles distingués. Ceci fournit l'isomorphisme recherché $L \simeq \Lambda(n)$.

Comme application de la discussion précédente, on obtient une globalisation de l'isomorphisme de pureté du Corollaire 7.5. Ainsi, si $i: Z \hookrightarrow S$ est une immersion fermée de schémas réguliers avec $Z$ partout de codimension $d$, on a un isomorphisme $i^{!} \Lambda_{S} \simeq \Lambda_{Z}(-d)[-2 d]$.

Remarque 11.3 - On peut aussi utiliser la Proposition 11.1 pour montrer que $\mathbf{D A}{ }^{\text {ét }}(S, \Lambda)$ est

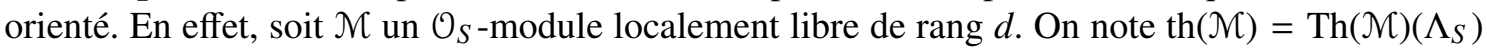
de sorte que $\operatorname{Th}(\mathcal{M}) \simeq \operatorname{th}(\mathcal{M}) \otimes-$. Nous allons construire un isomorphisme canonique

$$
\operatorname{hom}_{\mathbf{D A}^{\mathrm{et}}(S, \Lambda)}(\operatorname{th}(\mathcal{M}), \Lambda(d)[2 d]) \simeq \Lambda^{\pi_{0}(S)} .
$$

Lorsque $\mathcal{M}$ est libre, un isomorphisme (112) est fourni par la Proposition 11.1. Cet isomorphisme est indépendant de la trivialisation $\mathcal{M} \simeq \mathcal{O}_{S}^{\oplus d}$ choisie. (Pour démontrer cela, on remarque que les différentes trivialisations fournissent un morphisme de faisceaux de groupes $\mathbb{G L}_{S}(d) \longrightarrow \Lambda_{S}^{\times}$ du groupe linéaire vers le groupe des éléments inversibles de $\Lambda$ vu comme un schéma en groupe constant sur $S$. Or, un tel morphisme est nécessairement trivial puisque $\mathbb{G}_{L}(d)$ est connexe alors que $\Lambda_{S}^{\times}$est discret.)

Le cas général s'obtient par induction sur le nombre d'ouverts dans un recouvrement trivialisant de $\mathcal{M}$. Cette induction est possible par ce qui suit. Supposons que $S=U \cup V$ est un recouvrement ouvert tel que (112) est construit pour $U, V$ et $U \cap V$. La Proposition 11.1 entraîne facilement

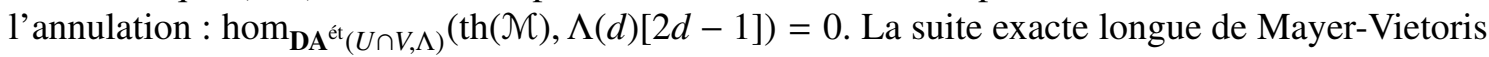
fournit alors une suite exacte

$$
0 \longrightarrow \operatorname{hom}_{\mathbf{D A}^{\text {st }}(S, \Lambda)}(\operatorname{th}(\mathcal{M}), \Lambda(d)[2 d]) \longrightarrow \Lambda^{\pi_{0}(U)} \bigoplus \Lambda^{\pi_{0}(V)} \longrightarrow \Lambda^{\pi_{0}(U \cap V)} .
$$

Ceci permet de déduire (112) pour $S$.

L'image de $1 \in \Lambda^{\pi_{0}(S)}$ par l'identification (112) fournit un isomorphisme canonique th( $(\mathcal{M}) \simeq$ $\Lambda(d)[2 d]$ dans $\mathbf{D} \mathbf{A}^{\text {ét }}(S, \Lambda)$. Le lecteur se convaincra facilement que cet isomorphisme est compatible aux changements de base.

Corollaire 11.4 - Soient $S$ un schéma régulier et $\Lambda$ un anneau de coefficients. On suppose que l'Hypothèse 7.3 est satisfaite et que $\Lambda$ est plat sur $\mathbb{Z}$. Alors, on a

$$
\operatorname{hom}_{\mathbf{D A}^{\text {et }}(S, \Lambda)}\left(\Lambda_{S}, \Lambda_{S}(1)[n]\right)=\left\{\begin{array}{ccc}
\mathcal{O}^{\times}(S) \otimes_{\mathbb{Z}} \Lambda & \text { si } & n=1, \\
0 & \text { si } & n \leq 0 .
\end{array}\right.
$$

Demonstration On ne restreint pas la généralité en supposant que $S$ est connexe. Soient $j: U \hookrightarrow$ $S$ l'inclusion d'un ouvert non vide. Montrons que le morphisme

$$
\operatorname{hom}_{\mathbf{D A}}{ }^{\text {et }(S, \Lambda)}\left(\Lambda_{S}, \Lambda_{S}(1)[n]\right) \longrightarrow \operatorname{hom}_{\mathbf{D A}^{\text {ét }}(U, \Lambda)}\left(\Lambda_{U}, \Lambda_{U}(1)[n]\right)
$$

est injectif si $n \leq 1$. Pour cela, on considère l'immersion fermée $i: Z \hookrightarrow U$ avec $Z=S-U$. On peut filtrer $Z$ par des sous-schémas fermés $\emptyset=Z_{0} \subset Z_{1} \subset \cdots \subset Z_{n}=Z$ de sorte que $Z_{i}-Z_{i-1}$ est régulier pour tout $1 \leq i \leq n$. Or, il est suffisant de montrer que le morphisme (113) est injectif pour les immersions ouvertes $S-Z_{i} \hookrightarrow S-Z_{i-1}$. Ceci nous ramène au cas où $Z$ est régulier de codimension $c \geq 1$. D'après le Corollaire 7.5 (et la Remarque 11.2), on a un isomorphisme de pureté $i^{!} \Lambda_{S} \simeq \Lambda_{Z}(-c)[-2 c]$. À l'aide du triangle distingué de localité (cf. [4, Prop. 1.4.9]), on déduit une suite exacte

$$
\operatorname{hom}\left(\Lambda_{Z}, \Lambda_{Z}(1-c)[n-2 c]\right) \longrightarrow \operatorname{hom}\left(\Lambda_{S}, \Lambda_{S}(1)[n]\right) \longrightarrow \operatorname{hom}\left(\Lambda_{S}, \Lambda_{S}(1)[n]\right)
$$


La Proposition 11.1 permet de conclure.

En passant à la limite suivant les ouverts non-vides $U \subset S$, on déduit de (113) un morphisme injectif

$$
\operatorname{hom}_{\mathbf{D A}^{\text {et }}(S, \Lambda)}\left(\Lambda_{S}, \Lambda_{S}(1)[n]\right) \longrightarrow \operatorname{hom}_{\mathbf{D A}^{\text {et }}(K, \Lambda)}\left(\Lambda_{K}, \Lambda_{K}(1)[n]\right)
$$

avec $K$ le corps des fonctions rationnelles sur $S$. Par ailleurs, si $K^{\text {parf }}$ désigne la clôture parfaite de $K$, on a des équivalences de catégories (cf. les Théorèmes 3.9 et B.1)

$$
\mathbf{D A}^{\text {ét }}(K, \Lambda) \simeq \mathbf{D A}^{\text {ét }}\left(K^{\text {parf }}, \Lambda\right) \simeq \mathbf{D M}^{\text {ét }}\left(K^{\text {parf }}, \Lambda\right) \text {. }
$$

En utilisant le « cancellation theorem » de Voevodsky [51] et le calcul de la cohomologie motivique en poids un [31, Th. 4.1 et Cor. 4.2] on déduit que

$$
\operatorname{hom}_{\mathbf{D M}^{\text {st }}\left(K^{\text {parf }, \Lambda)}\right.}(\Lambda, \Lambda(1)[n])=\left\{\begin{array}{ccc}
K^{\times} \otimes_{\mathbb{Z}} \Lambda & \text { si } & n=1, \\
0 & \text { si } & n \leq 0 .
\end{array}\right.
$$

(Ci-dessus, nous avons utiliser que l'exposant caractéristique de $K$ est inversible dans $\Lambda$ pour déduire que $K^{\times} \otimes_{\mathbb{Z}} \Lambda \simeq\left(K^{\text {parf }}\right)^{\times} \otimes_{\mathbb{Z}} \Lambda$.) À ce stade, le corollaire est démontré lorsque $n \leq 0$.

Dans la suite, on supposera que $n=1$. L'argument précédent montre que (113) est un isomorphisme lorsque $Z \subset S$ est partout de codimension plus grande ou égale à 2. (En effet, la Proposition 11.1 entraîne que hom $\mathbf{D A}^{\text {st }(Z, \Lambda)}\left(\Lambda_{Z}, \Lambda_{Z}(1-c)[n-2 c+1]\right)=0$ pour $c \geq 2$ et $n \leq 1$.)

Soit $x \in S$ un point de codimension 1 et notons $Z=\overline{\{x\}}$. Quitte à remplacer $S$ par le complémentaire d'un fermé de codimension plus grande ou égale à 2, on peut supposer que $Z$ est lisse. Par pureté (cf. le Corollaire 7.5) et le triangle de localité (cf. [4, Prop. 1.4.9]), on a une suite exacte

$$
\operatorname{hom}_{\mathbf{D A}^{\mathrm{et}}(S, \Lambda)}\left(\Lambda_{S}, \Lambda_{S}(1)[1]\right) \longrightarrow \operatorname{hom}_{\mathbf{D A}^{\mathrm{et}}(U, \Lambda)}\left(\Lambda_{U}, \Lambda_{U}(1)[1]\right) \stackrel{\partial_{Z}}{\longrightarrow} \operatorname{hom}_{\mathbf{D A}^{\mathrm{et}}(Z, \Lambda)}\left(\Lambda_{Z}, \Lambda_{Z}\right)
$$

Ceci montre que hom $\mathbf{D A}^{\text {et }(S, \Lambda)}\left(\Lambda_{S}, \Lambda_{S}(1)[1]\right)$ est contenu dans le noyau du morphisme

$$
\partial_{x}: \operatorname{hom}_{\mathbf{D A}^{e t}(K, \Lambda)}\left(\Lambda_{K}, \Lambda_{K}(1)[1]\right) \longrightarrow \operatorname{hom}_{\mathbf{D A}^{e t}(K(x), \Lambda)}(\Lambda, \Lambda) \simeq \Lambda .
$$

Modulo l'identification hom ${ }_{\mathbf{D A}^{\text {st }}(K, \Lambda)}\left(\Lambda_{K}, \Lambda_{K}(1)[1]\right) \simeq K^{\times} \otimes_{\mathbb{Z}} \Lambda$, ce morphisme est donnée par la valuation normalisée $v_{x}$ associée à l'anneau de valuation $\mathcal{O}_{S, x} \subset K$. Mais alors, on a une suite exacte bien connue

$$
0 \longrightarrow \mathcal{O}^{\times}(S) \otimes \Lambda \longrightarrow K \otimes \Lambda \stackrel{\sum_{x} v_{x}}{\longrightarrow} \bigoplus_{x \in S^{(1)}} \Lambda
$$

où $S^{(1)}$ désigne l'ensemble des points de codimension 1 dans $S$. On en déduit une inclusion

$$
\operatorname{hom}_{\mathbf{D A}^{\text {et }(S, \Lambda)}}\left(\Lambda_{S}, \Lambda_{S}(1)[1]\right) \hookrightarrow \mathcal{O}^{\times}(S) \otimes_{\mathbb{Z}} \Lambda .
$$

Par ailleurs, il existe un homomorphisme naturel $\mathcal{O}^{\times}(S) \otimes_{\mathbb{Z}} \Lambda \longrightarrow \operatorname{hom}_{\mathbf{D A}^{\text {et }}(S, \Lambda)}\left(\Lambda_{S}, \Lambda_{S}(1)[1]\right)$. Il provient du fait que dans $\mathbf{D M}^{\mathrm{eff}}(S, \Lambda)$ on a un isomorphisme $\Lambda_{t r}\left(\mathbb{G m}_{S}, 1\right) \simeq \mathcal{O}^{\times} \otimes_{\mathbb{Z}} \Lambda$. Ceci permet de conclure.

On commence maintenant la construction du système de spécialisation logarithmique. Soit $S$ un schéma de base régulier. (Plus tard, $S$ sera le spectre d'un anneau de valuation discrète, hensélien et excellent.) On dispose d'un morphisme dans $\mathbf{D A}{ }^{\text {ét }}\left(\mathbb{G}_{S}, \Lambda\right)$

$$
e_{\mathrm{K}}: \Lambda_{\mathbb{G} \mathrm{m}_{S}}(-1)[-1] \longrightarrow \Lambda_{\mathbb{G} \mathrm{m}_{S}}
$$

définie comme dans [5, Déf. 3.6.22]. Lorsque $\Lambda$ est plat sur $\mathbb{Z}$, ce morphisme correspond à l'indeterminée $\pi \in \mathcal{O}^{\times}\left(\mathbb{G m}_{S}\right) \simeq \operatorname{hom}_{\mathbf{D A}^{\mathrm{et}}\left(\mathbb{G m}_{S}, \Lambda\right)}(\Lambda(-1)[-1], \Lambda)$ telle que $\mathbb{A}_{S}^{1}=S[\pi]$. On forme un triangle distingué

$$
\Lambda_{\mathbb{G} \mathrm{m}_{S}} \longrightarrow \mathscr{K}_{S} \longrightarrow \Lambda_{\mathbb{G} \mathrm{m}_{S}}(-1) \stackrel{-e_{\mathrm{K}}[1]}{\longrightarrow} \Lambda_{\mathbb{G} \mathrm{m}_{S}}[1] .
$$

(À une rotation près, c'est le triangle distingué de [5, Lem. 3.6.28].) Le motif $\mathscr{K}_{S}$ est appelé l'extension de Kummer. Lorsqu'il n'y a pas de confusion possible, on le notera $\mathscr{K}$ au lieu de $\mathscr{K}_{S}$.

Lemme 11.5 - On suppose que $\Lambda$ est une $\mathbb{Q}$-algèbre. Le triangle distingué (115) satisfait aux conditions de [5, Hyp. 3.6.1]. 
Demonstration Les objets $\Lambda_{\mathbb{G}_{S}}$ et $\Lambda_{\mathbb{G} \mathrm{m}_{S}}(-1)$ sont clairement inversibles. Aussi, la permutation des facteurs agit par l'identité sur $\Lambda_{\mathbb{G m}_{S}} \otimes \Lambda_{\mathbb{G}_{S}}$ (c'est clair!) et $\Lambda_{\mathbb{G m}_{S}}(-1) \otimes \Lambda_{\mathbb{G} \mathrm{m}_{S}}(-1)$ (d'après la Proposition 2.10). Il vient $\operatorname{Alt}^{2}\left(\Lambda_{\mathbb{G} \mathrm{m}_{S}}\right)$ et $\operatorname{Alt}^{2}\left(\Lambda_{\mathbb{G} \mathrm{m}_{S}}(-1)\right)$ sont nuls.

Les groupes hom ${ }_{\mathbf{D A}^{\text {st }}\left(\mathrm{Gm}_{s}, \Lambda\right)}(\Lambda, \Lambda(-n)[k])$ sont nuls pour $n \geq 1$ et $k \in\{-1,0\}$ comme le montre la Proposition 11.1, (b). De même, le groupe $\operatorname{hom}_{\mathbf{D A}^{\text {et }}\left(\mathbb{G}_{S}, \Lambda\right)}(\Lambda(-1), \Lambda)$ est nul d'après le Corollaire

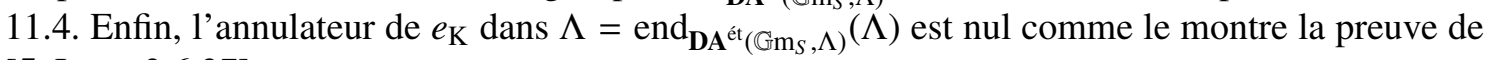
[5, Lem. 3.6.27].

Lorsque $\Lambda$ est une $\mathbb{Q}$-algèbre, nous disposons donc des résultats et constructions de $[\mathbf{5}, \S 3.6 .1]$ pour le triangle distingué (115). En particulier, ce triangle est unique à un unique isomorphisme près d'après [5, Lem. 3.6.3].

Definition 11.6 - On suppose que $\Lambda$ est une $\mathbb{Q}$-algèbre. Le logarithme $\mathscr{L} o g^{\vee}=\operatorname{Sym}^{\infty}(\mathscr{K})$ est la colimite homotopique de la $\mathbb{N}$-suite $\left(\mathscr{L} \circ g_{n}^{\vee}\right)_{n \in \mathbb{N}}$ dans $\mathbf{D A}^{\mathrm{e} t}\left(\mathbb{G}_{S}, \Lambda\right)$ donnée par $\mathscr{L} \operatorname{og}_{n}^{\vee}=$ $\operatorname{Sym}^{n}(\mathscr{K})$ (cf. [5, Déf. 3.6.29]). D'après [5, Cor. 3.6.19], cet objet est bien défini à un unique isomorphisme près. De plus, il est naturellement muni d'une structure d'algèbre associative, commutative et unitaire.

D’une importance capitale pour la suite est le triangle distingué (cf. [5, Cor. 3.6.21]) :

$$
\Lambda(0) \longrightarrow \mathscr{L}_{o g}{ }^{\vee} \stackrel{\mathrm{N}}{\longrightarrow} \mathscr{L}_{\operatorname{og}^{\vee}}(-1) \longrightarrow \Lambda(0)[1] .
$$

Malheureusement, une erreur de «calcul » s'est incrustée lors de sa construction dans [5, §3.6]. Nous devons la corriger pour le bon fonctionnement de la preuve du théorème principal de cette section (à savoir le Théorème 11.17). L'origine de cette erreur se trouve tout au début de l'Étape 4 de la preuve de [5, Th. 3.6.10]. Nous y affirmons à tort que le carré évident

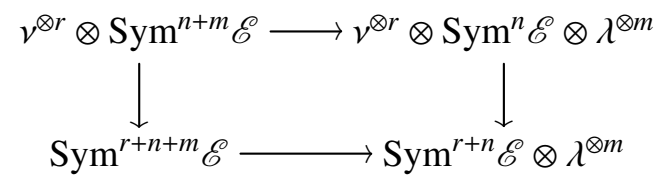

est commutatif. En fait, on a plutôt la propriété suivante.

Lemme 11.7 - On reprend les hypothèses et les notations de [5, §3.6.1], et notamment celles de [5, Th. 3.6.10]. Alors, le carré (117) commute au facteur $C_{n+m}^{n} / C_{r+n+m}^{r+n}$ près. Plus précisément, si on multiplie dans (117) la flèche horizontale supérieure par $C_{n+m}^{n}$ et la flèche horizontale inférieure par $C_{r+n+m}^{r+n}$, on obtient un carré commutatif.

Demonstration Il s'agit de comparer les deux flèches entre $v^{\otimes r} \otimes \operatorname{Sym}^{n+m} \mathscr{E}$ et $\operatorname{Sym}^{r+n} \mathscr{E} \otimes \lambda^{\otimes m}$ obtenues en prenant les deux compositions possibles dans (117). Il suffira de les comparer après composition à droite et à gauche par

$$
v^{\otimes r} \otimes \mathscr{E}^{\otimes n+m} \longrightarrow v^{\otimes r} \otimes \operatorname{Sym}^{n+m} \mathscr{E} \quad \text { et } \quad \operatorname{Sym}^{r+n} \mathscr{E} \otimes \lambda^{\otimes m} \hookrightarrow \mathscr{E}^{\otimes r+n} \otimes \lambda^{\otimes m} .
$$

Les deux flèches à comparer sont alors données comme suit. Celle obtenue en composant dans (117) la flèche horizontale supérieure avec la flèche verticale de droite est donnée par :

$$
\left(\left(\frac{1}{(r+n) !} \sum_{\sigma^{\prime} \in \Sigma_{r+n}} \sigma^{\prime}\right) \otimes \mathrm{id}\right) \circ\left(a^{\otimes r} \otimes \mathrm{id}\right) \circ\left(\mathrm{id} \otimes b^{\otimes m}\right) \circ\left(\mathrm{id} \otimes\left(\frac{1}{(n+m) !} \sum_{\sigma^{\prime \prime} \in \Sigma_{n+m}} \sigma^{\prime \prime}\right)\right) .
$$

Celle obtenue en composant dans (117) la flèche verticale de gauche avec la flèche horizontale inférieure est donnée par :

$$
\left(\mathrm{id} \otimes b^{\otimes m}\right) \circ\left(\frac{1}{(r+n+m) !} \sum_{\sigma \in \Sigma_{r+n+m-1}} \sigma\right) \circ\left(a^{\otimes r} \otimes \mathrm{id}\right) .
$$


Il est clair que (118) est aussi égale à :

$$
\left(\mathrm{id} \otimes b^{\otimes m}\right) \circ\left(\left(\frac{1}{(r+n)^{!}} \sum_{\sigma^{\prime} \in \Sigma_{r+n}} \sigma^{\prime}\right) \otimes \mathrm{id}\right) \circ\left(\mathrm{id} \otimes\left(\frac{1}{(n+m) !} \sum_{\sigma^{\prime \prime} \in \Sigma_{n+m}} \sigma^{\prime \prime}\right)\right) \circ\left(a^{\otimes r} \otimes \mathrm{id}\right) .
$$

D'autre part, $\left(\mathrm{id} \otimes b^{\otimes m}\right) \circ \sigma \circ\left(a^{\otimes r} \otimes \mathrm{id}\right)=0$ si $\sigma(\llbracket 1, r \rrbracket) \not \subset \llbracket 1, r+n \rrbracket$. On peut donc réécrire (119) de la manière suivante :

$$
\left(\mathrm{id} \otimes b^{\otimes m}\right) \circ\left(\frac{1}{(r+n+m) !} \sum_{\sigma \in \Sigma_{r+n+m-1}, \sigma(\llbracket 1, r \rrbracket \subset \llbracket 1, r+n \rrbracket} \sigma\right) \circ\left(a^{\otimes r} \otimes \mathrm{id}\right) .
$$

Or, l'application $\Sigma_{r+n} \times \Sigma_{n+m} \longrightarrow \Sigma_{r+n+m}$, donnée par $\left(\sigma^{\prime}, \sigma^{\prime \prime}\right) \rightsquigarrow\left(\sigma^{\prime} \times \operatorname{id}_{m}\right) \circ\left(\operatorname{id}_{r} \times \sigma^{\prime \prime}\right)$, induit une surjection de $\Sigma_{r+n} \times \Sigma_{n+m}$ sur l'ensemble des $\sigma \in \Sigma_{r+n+m}$ tels que $\sigma(\llbracket 1, r \rrbracket) \subset \llbracket 1, r+n \rrbracket$. Le cardinal des fibres est constant et égal à $n$ !. Il en découle que (118) multipliée par $(r+n) !(n+m)$ ! vaut (119) multipliée par $n !(r+n+m)$ !. Or, on a :

$$
\frac{(r+n) !(n+m) !}{n !(r+n+m) !}=C_{n+m}^{n} / C_{r+n+m}^{r+n}
$$

Le lemme est démontré.

On peut maintenant corriger l'énoncé de [5, Th. 3.6.10]. L'erreur décrite ci-dessus n'affecte que la partie 2 de cet énoncé. Toutefois, il sera plus pratique pour la suite de modifier également le triangle distingué de la partie 1 . On retient donc l'énoncé suivant.

Theoreme 11.8 - On reprend les hypothèses et les notations de [5, §3.6.1], et notamment celles de [5, Th. 3.6.10].

(a) Pour $(m, n) \in(\mathbb{N}-\{0\})^{2}$, il existe un unique triangle distingué :

$$
v^{\otimes n} \otimes \operatorname{Sym}^{m-1} \mathscr{E} \stackrel{a_{n, m-1}}{\longrightarrow} \operatorname{Sym}^{n+m-1} \mathscr{E} \stackrel{\mathrm{C}_{n+m-1}^{n-1} \cdot b_{n-1, m}}{\longrightarrow} \operatorname{Sym}^{n-1} \mathscr{E} \otimes \lambda^{m} \longrightarrow
$$

(Ci-dessus $a_{n, m-1}$ et $b_{n, m-1}$ sont les morphismes évidents induits par $a: v \longrightarrow \mathscr{E}$ et $b$ : $\mathscr{E} \longrightarrow \lambda$.)

(b) Pour $(m, n, r) \in(\mathbb{N}-\{0\})^{2} \times \mathbb{N}$, on a un morphisme de triangles distingués :

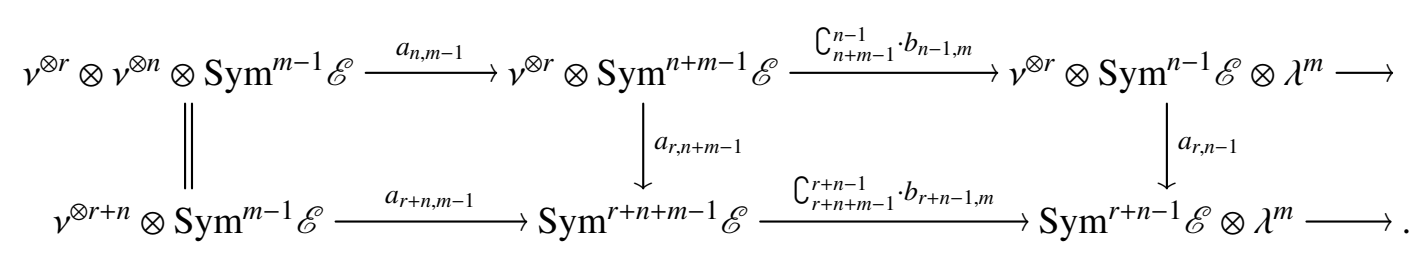

Demonstration D'après [5, Th. 3.6.10], il existe un triangle distingué comme dans (a) au facteur $C_{n+m-1}^{n-1}$ près. Comme $\mathcal{T}$ est supposée $\mathbb{Q}$-linéaire, ceci démontre la partie (a). Pour la partie (b), il faut reprendre l'Étape 4 de la preuve de [5, Th. 3.6.10] en partant d'un carré commutatif comme celui fourni par le Lemme 11.7.

Retournons à la situation qui nous intéresse et supposons que $\Lambda$ est une $\mathbb{Q}$-algèbre. D'après le Théorème 11.8, on a des morphismes de triangles distingués dans $\mathbf{D} \mathbf{A}^{\text {ét }}\left(\mathbb{G}_{S}, \Lambda\right)$ :

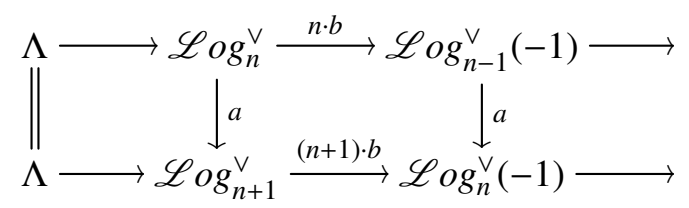

avec $a$ et $b$ les morphismes déduits de $\Lambda(0) \longrightarrow \mathscr{K}$ et $\mathscr{K} \longrightarrow \Lambda(-1)$. Le triangle distingué (116) est obtenu en passant à la colimite homotopique suivant $n \in \mathbb{N}$. Même si la construction du triangle (116) dans [5, §3.6] est erronée, la preuve de [5, Cor. 3.6.21] est correcte lorsqu' on utilise les 
triangles distingués du Théorème 11.8. Le triangle (116) est donc bien défini à un unique isomorphisme près et en particulier le morphisme $\mathrm{N}: \mathscr{L}_{o g}{ }^{\vee} \longrightarrow \mathscr{L}_{o g}{ }^{\vee}(-1)$ est uniquement déterminé. (Voir aussi le Lemme 11.9 ci-dessous.)

Plus généralement, le Théorème 11.8 fournit des morphismes de triangles distingués

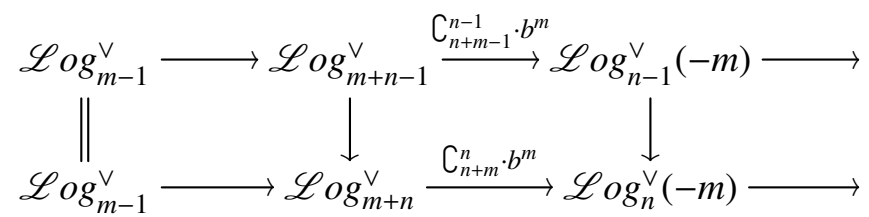

En passant à la colimite, on obtient les triangles distingués

$$
\mathscr{L}_{o g}^{\vee} \longrightarrow \mathscr{L}_{m-1}{ }^{\vee} \stackrel{\mathrm{N}_{m}}{\longrightarrow} \mathscr{L}_{o g^{\vee}}(-m) \longrightarrow \mathscr{L}_{o g_{m-1}^{\vee}}[1] .
$$

On a également le résultat suivant.

LEMME 11.9 - Les triangles distingués (120) sont bien définis à un unique isomorphisme près. En particulier, les morphismes $\mathrm{N}_{m}$ sont uniquement déterminés.

Demonstration On montre d'abord que la colimite homotopique des morphismes $C_{n+m}^{n} \cdot b^{m}$ :

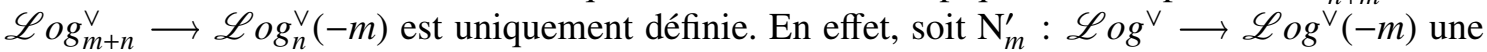
autre colimite. Alors, $\epsilon=\mathrm{N}_{m}^{\prime}-\mathrm{N}_{n}$ est une flèche fantôme entre $\mathscr{L} o g^{\vee}$ et $\mathscr{L} o g^{\vee}(-m)$ au sens que la composition de

$$
\mathscr{L} \operatorname{og}_{r}^{\vee} \longrightarrow \mathscr{L}_{o g}{ }^{\vee} \stackrel{\epsilon}{\longrightarrow} \mathscr{L}_{o g}{ }^{\vee}(-m)
$$

est nulle pour tout $r \in \mathbb{N}$. D' après [5, Prop. 3.6.18], ${ }^{(9)}$ la flèche $\epsilon$ est nécessairement nulle puisque $\mathscr{L} \operatorname{og}^{\vee}(-m)$ est un module unitaire sur l'algèbre $\mathscr{L} o g^{\vee}$.

Il reste à voir que la flèche connectante $\mathscr{L} \operatorname{og}^{\vee}(-m) \longrightarrow \mathscr{L}^{\circ} g_{m-1}^{\vee}[1]$ est unique. Pour cela, on raisonne comme dans la preuve de [5, Cor. 3.6.21]. On voit alors qu'il est suffisant de montrer que hom ${ }_{\mathbf{D A}^{\mathrm{et}}\left(\mathbb{G m}_{S}, \Lambda\right)}\left(\mathscr{L} \operatorname{og}_{m-1}^{\vee}, \mathscr{L}_{o g^{\vee}}(-m)[r]\right)=0$ pour tout $r \in \mathbb{Z}$. Étant donné que $\mathscr{L} \operatorname{og}_{m-1}^{\vee}$ est dans la sous-catégorie triangulée engendrée par les motifs de Tate $\Lambda_{\mathbb{G m}_{S}}(-i)$ pour $0 \leq i \leq m-1$, on se ramène à montrer que $\operatorname{hom}_{\mathbf{D A}^{\text {st }}\left(\mathbb{G} \mathrm{m}_{S}, \Lambda\right)}\left(\Lambda, \mathscr{L}_{o g^{\vee}}(i-m)[r]\right)=0$ pour $i<m$. Ceci est une conséquence de la Proposition 11.1, (b).

LEMME 11.10 - On a la relation $m ! \cdot \mathrm{N}_{m}=\mathrm{N}^{\circ m}$.

Demonstration En effet, il est clair que $\mathrm{N}^{\mathrm{om}}$ est donné par la colimite homotopique de la composition de

$$
\mathscr{L} o g_{m+n}^{\vee} \stackrel{(m+n) b}{\longrightarrow} \mathscr{L}_{o g_{m-n-1}^{\vee}}^{\vee}(-1) \stackrel{(m+n-1) b}{\longrightarrow} \cdots \stackrel{(n+1) b}{\longrightarrow} \mathscr{L}_{o g_{n}^{\vee}}^{\vee}(-m) .
$$

Le résultat découle maintenant de la formule $(m+n) \cdots(1+n)=m ! \complement_{n+m}^{n}$ et de l'unicité des colimites homotopiques en question.

Lemme 11.11 - On suppose que $\Lambda$ est une $\mathbb{Q}$-algèbre. On note $q: \mathbb{G}_{S} \longrightarrow S$ la projection canonique. On a des triangles distingués canoniques dans $\mathbf{D} \mathbf{A}^{\mathrm{e}}(S, \Lambda)$ :

$$
\Lambda_{S}(-n-1)[-2] \stackrel{0}{\longrightarrow} \Lambda_{S}(0) \longrightarrow q_{*} \mathscr{L}_{o g}^{\vee} \longrightarrow \Lambda_{S}(-n-1)[-1] .
$$

De plus, les diagrammes suivants commutent :

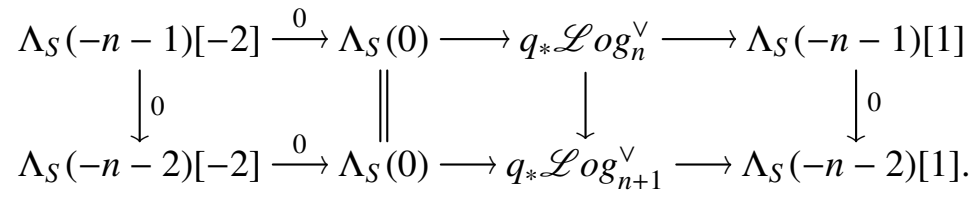

Demonstration C'est la même preuve que [5, Lem. 3.6.30].

9. La preuve de [5, Prop. 3.6.18] contient une erreur de frappe à la quatrième ligne qui risque de compromettre sa compréhensibilité : il faut remplacer $\left(\operatorname{Sym}^{n} \mathscr{E}\right) \otimes \mathscr{C} \longrightarrow\left(\operatorname{Sym}^{n} \mathscr{E}\right) \otimes \mathscr{C} \operatorname{par}\left(\operatorname{Sym}^{n} \mathscr{E}\right) \otimes \mathscr{C} \longrightarrow\left(\operatorname{Sym}^{n+1} \mathscr{E}\right) \otimes \mathscr{C}$. 
Corollaire 11.12 - Gardons les hypothèses et les notations $d u$ Lemme 11.11. La composition de $\Lambda(0) \longrightarrow q_{*} \Lambda(0) \longrightarrow q_{*} \mathscr{L}_{\text {og }}^{\vee}$ est un isomorphisme.

On suppose que $\Lambda$ est une $\mathbb{Q}$-algèbre. Soit $f: X \longrightarrow \mathbb{A}_{S}^{1}$ un morphisme de $S$-schémas quasiprojectifs. On définit un foncteur $\log _{f}: \mathbf{D A}^{\mathrm{et}}\left(X_{\eta}, \Lambda\right) \longrightarrow \mathbf{D A}^{\mathrm{ét}}\left(X_{\sigma}, \Lambda\right)$ par

$$
\log _{f}(A)=\chi_{f}\left(A \otimes f_{\eta}^{*} \mathscr{L} \circ g^{\vee}\right)=i^{*} j_{*}\left(A \otimes f_{\eta}^{*} \mathscr{L} \circ g^{\vee}\right)
$$

Ceci définit un système de spécialisation de base $\left(\mathbb{A}_{S}^{1}, j, i\right)$. En utilisant le triangle distingué (116), on obtient un triangle distingué, dit de monodromie :

$$
\log _{f}(A)(-1)[-1] \longrightarrow \chi_{f}(A) \longrightarrow \log _{f}(A) \stackrel{\mathrm{N}}{\longrightarrow} \log _{f}(A)(-1)
$$

La transformation naturelle $\mathrm{N}: \log _{f} \longrightarrow \log _{f}(-1)$ est appelée l'opérateur de monodromie.

Theoreme 11.13 - Soient $S$ un schéma de base de dimension de Krull finie et $\Lambda$ une $\mathbb{Q}$-algèbre. On se donne un morphisme de $S$-schémas quasi-projectifs $f: X \longrightarrow \mathbb{A}_{S}^{1}$ avec $X$ régulier. On suppose que $\left(X_{\sigma}\right)_{\text {red }}$ est régulier. Alors, la composition de

$$
\Lambda_{X_{\sigma}} \longrightarrow \chi_{f} \Lambda_{X_{\eta}} \longrightarrow \log _{f} \Lambda_{X_{\eta}}
$$

est un isomorphisme dans $\mathbf{D} \mathbf{A}^{\mathrm{t}}\left(X_{\sigma}, \Lambda\right)$.

Demonstration La preuve est identique à celle du Théorème 10.2. On peut supposer que $S$ est régulier. Soit $t: X \longrightarrow W$ une immersion fermée dans un $\mathbb{A}_{S}^{1}$-schéma lisse $g: W \longrightarrow \mathbb{A}_{S}^{1}$. Le morphisme de changement de base $g_{\sigma}^{*} \log _{\text {id }} \longrightarrow \log _{g} g_{\eta}^{*}$ est inversible puisque $g$ est lisse. D'après le Corollaire 11.12, le théorème est donc vrai pour $W$. Il suffit alors de montrer que le morphisme naturel $t_{\sigma}^{*} \log _{g} \Lambda_{W_{\eta}} \longrightarrow \log _{f} t_{\eta}^{*} \Lambda_{W_{\eta}}$ est inversible. Il est donné par $t_{\sigma}^{*} \chi_{g} g_{\eta}^{*} \mathscr{L}_{o g} \vee \longrightarrow \chi_{f} f_{\eta}^{*} \mathscr{L}_{o g} \vee$. Or, $\mathscr{L} \circ g^{\vee} \in \mathbf{D A}^{\mathrm{e}}\left(\mathbb{G m}_{S}, \Lambda\right)$ est dans la plus petite sous-catégorie triangulée stable par sommes infinies et contenant les motifs de Tate $\Lambda_{\mathbb{G} \mathrm{m}_{S}}(-n)$, pour $n \in \mathbb{N}$. Il suffit donc de montrer que $t^{*} j_{*} \Lambda \longrightarrow j_{*} t_{\eta}^{*} \Lambda$ est inversible. Ceci découle de la Proposition 10.3.

À partir de maintenant, on suppose que $S=\operatorname{Spec}(R)$ avec $R$ un anneau de valuation discrète, hensélien et excellent. On fixe une uniformisante $\pi \in R$. Ceci permet de restreindre le système de spécialisation log à la base $(S, \eta, \sigma)$.

Theoreme 11.14 - On suppose que $\Lambda$ est une $\mathbb{Q}$-algèbre. Il existe un isomorphisme canonique de systèmes spécialisation log $\stackrel{\sim}{\longrightarrow} \Upsilon$ au-dessus de $(S, \eta, \sigma)$.

Demonstration Il s'agit de calquer la preuve de [5, Th. 3.6.44]. Appelons $\mathscr{U}=\left(p_{\Delta}\right)_{\sharp} \theta_{*}^{\mathscr{A}} \Lambda \in$ $\mathbf{D A}^{\text {ét }}\left(\mathbb{G}_{S}, \Lambda\right)$. (cf. (89).) La première condition de [5, Hyp. 3.6.40] est clairement satisfaite. La preuve de [5, Lem. 3.6.41] fournit alors un isomorphisme naturel $\Upsilon_{f} \simeq \chi_{f}\left(-\otimes f_{\eta}^{*} \mathscr{U}\right)$ pour tout morphisme de $S$-schémas quasi-projectifs $f: X \longrightarrow \mathbb{A}_{S}^{1}$. D'autre part, on dispose d'un morphisme canonique $\ell_{1}: \mathscr{K} \longrightarrow \mathscr{U}$. Il est construit comme dans [5, pp. 134 à 136]. Étant donné que $\mathscr{U}$ est une algèbre associative et unitaire, on peut appliquer [5, Cor. 3.6.19] pour déduire un morphisme $\ell: \mathscr{L}_{o g} \vee \longrightarrow \mathscr{U}$. Ceci fournit un morphisme de systèmes de spécialisation log $\longrightarrow \Upsilon$. Pour montrer que c'est un isomorphisme au-dessus de $(S, \eta, \sigma)$, on applique [5, Th. 3.3.47] et les Théorèmes 10.2 et 11.13 .

Remarque 11.15 - La preuve du Théorème 11.14 que nous venons de présenter n'est pas la meilleure. Elle est non élémentaire puisqu'elle repose sur des techniques de résolution des singularités. On peut obtenir une preuve plus élémentaire du Théorème 11.14 en montrant directement que le morphisme $\mathscr{L}_{o g}{ }^{\vee} \longrightarrow \mathscr{U}$ est inversible. C'est ce que fait Levine dans [30]. Nous nous sommes retenus d'utiliser la méthode de Levine pour ne pas allonger encore plus la discussion.

Theoreme 11.16 - On suppose que $\Lambda$ est une $\mathbb{Q}$-algèbre. Soient $f: X \longrightarrow S$ un $S$-schéma quasi-projectif et $A \in \mathbf{D A}^{\mathrm{e} t}\left(X_{\eta}, \Lambda\right)$. Il existe alors un triangle distingué, dit de monodromie,

$$
\Upsilon_{f}(A)(-1)[-1] \longrightarrow \chi_{f}(A) \longrightarrow \Upsilon_{f}(A) \stackrel{\mathrm{N}}{\longrightarrow} \Upsilon_{f}(A)(-1)
$$


Il est compatible à la dualité au sens de [5, Th. 3.6.46]. De plus, si A est constructible, alors l'opérateur $\mathrm{N}: \Upsilon_{f}(A) \longrightarrow \Upsilon_{f}(A)(-1)$ est nilpotent.

Demonstration Le triangle de monodromie est obtenu à partir (122) via l'isomorphisme log $\simeq$ $\Upsilon$. La nilpotence de l'opérateur de monodromie est une conséquence de [5, Lem. 3.6.48] et du Théorème 10.9, (ii). La compatibilité avec la dualité se vérifie au niveau de log comme dans la preuve de [5, Th. 3.6.39]. Vue l'erreur dans [5, Th. 3.6.10] corrigée ci-dessus, il est en fait nécessaire d'apporter quelques changements dans l'Étape 1 de la preuve de [5, Th. 3.6.39]. En effet, avec la bonne définition de $\mathrm{N}: \mathscr{L}_{o g}{ }^{\vee} \longrightarrow \mathscr{L}_{o g^{\vee}}(-1)$, le second diagramme de la page 131 de [5] ne commute pas. On doit donc raisonner de la manière suivante. On fixe $(m, n) \in(\mathbb{N}-\{0\})^{2}$ et on remplace le premier diagramme de la page 131 de [5] par :

$$
\begin{aligned}
& \chi_{\mathrm{id}}\left(\mathscr{L} o g_{m-1}^{\vee} \otimes \mathrm{Alt}^{2} \mathscr{K} \otimes \mathscr{L}_{o g_{n-1}^{\vee}}^{\vee}\right) \longrightarrow \chi_{\mathrm{id}}\left(\mathscr{L} \circ g_{m}^{\vee} \otimes \mathscr{L} \circ g_{n}\right) \stackrel{(m \cdot b) \otimes \mathrm{id}}{\longrightarrow} \chi_{\mathrm{id}}\left(\mathscr{L} \circ g_{m-1}(-1) \otimes \mathscr{L} \circ g_{n}^{\vee}\right) \\
& \downarrow \text { id } \otimes(n \cdot b) \\
& \chi_{\mathrm{id}}\left(\mathscr{L} \operatorname{og}_{m}^{\vee} \otimes \mathscr{L}_{o g_{n-1}^{\vee}}^{\vee}(-1)\right) \longrightarrow \chi_{\mathrm{id}}\left(\mathscr{L} \operatorname{og}^{\vee}(-1)\right) \simeq \Lambda(-1) \text {. }
\end{aligned}
$$

Par un passage à la limite suivant $(m, n) \in(\mathbb{N}-\{0\})^{2}$, on voit qu'il est suffisant de montrer que les deux compositions possibles du diagramme ci-dessus sont égales à un facteur $(-1)$ près. On peut bien entendu se limiter au cas $n=m$ ce qui nous permet d'éliminer les facteurs $m$ et $n$ multipliant les morphismes évidents id $\otimes b$ et $b \otimes$ id dans le diagramme ci-dessus. Ensuite, on utilise [5, Lem. 3.6.13] pour obtenir le diagramme commutatif :

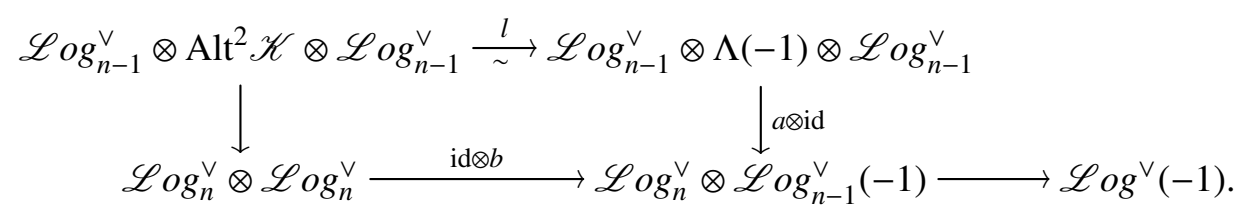

Le reste de l'Étape 1 de la preuve de [5, Th. 3.6.39] est valable avec les modifications évidentes qui consistent à remplacer certains « $\mathscr{L}$ og $\vee$ » par « $\mathscr{L} o g_{n}^{\vee} »$ ou $« \mathscr{L} o g_{n-1}^{\vee}$ ».

Jusqu'à la fin de la section, nous supposerons que $R$ contient les $n$-ièmes racines de l'unité pour $n$ premier à $p$. Ceci entraîne que $\widetilde{K}=K\left[\pi^{1 / n} \mid n \in \mathbb{N}^{\prime \times}\right]$ est une extension galoisienne de $K$ et son groupe de Galois s'identifie à $\widehat{\mathbb{Z}}^{\prime}(1)$ via le caractère cyclotomique qui envoie $\sigma \in \operatorname{Gal}(\widetilde{K} / K)$ sur $\left(\sigma\left(\pi^{1 / n}\right) / \pi^{1 / n}\right)_{n \in \mathbb{N}^{\prime} \times}$. L'anneau $\Lambda$ sera fixé et nous supposerons que l'Hypothèse 7.3 est satisfaite. Enfin, on se donne un idéal $J \subset \Lambda$ tel que $\Lambda / J$ est de torsion (en tant que $\mathbb{Z}$-module).

Soient $f: X \longrightarrow S$ un $S$-schéma quasi-projectif et $A$ un objet de $\hat{\mathbf{D}}_{\mathrm{ct}}^{\text {et }}\left(X_{\eta}, \Lambda_{J} \otimes \mathbb{Q}\right)$. Alors, le groupe $\widehat{\mathbb{Z}}^{\prime}(1)$ agit naturellement sur $\Psi_{f}^{\bmod }(A)=\widetilde{i}^{*} \widetilde{j}_{*}\left(A_{\mid X_{\tilde{\eta}}}\right)$ de la manière suivante. Fixons $\xi \in \widehat{\mathbb{Z}}^{\prime}(1)$ et notons $\operatorname{Spec}(\xi)$ les automorphismes de $\widetilde{\eta}$ et $\widetilde{S}$ déduit de $\xi$. Par changement de base, on obtient un diagramme commutatif aux carrés cartésiens

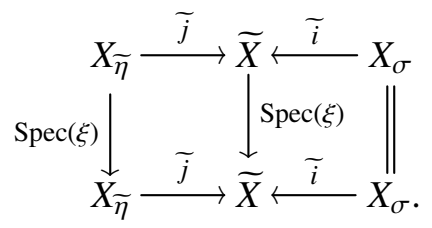

L'action de $\xi \operatorname{sur} \Psi_{f}^{\bmod }(A)$ est alors définie par la composition de

$$
\widetilde{i^{*}} \widetilde{j}_{*} A_{\mid X_{\tilde{\eta}}} \simeq \operatorname{id}^{*} i^{*} \widetilde{j}_{*} A_{\mid X_{\widetilde{\eta}}} \stackrel{\sim}{\longrightarrow} \widetilde{i^{*}} \operatorname{Spec}(\xi)^{*} \widetilde{j_{*}} A_{\mid X_{\tilde{\eta}}} \stackrel{\sim}{\longrightarrow} \widetilde{i}^{*} \widetilde{j_{*}} \operatorname{Spec}(\xi)^{*} A_{\mid X_{\tilde{\eta}}} \simeq \widetilde{i}^{*} \widetilde{j}_{*} A_{\mid X_{\bar{\eta}}} .
$$

C'est l'action par monodromie sur $\Psi_{f}^{\text {mod }}(A)$. Le résultat principal de cette section s'énonce alors comme suit. 
Theoreme 11.17 - Soient $M \in \mathbf{D A}^{\mathrm{t}}(S, \Lambda \otimes \mathbb{Q})$ un motif constructible et $\xi \in \hat{\mathbb{Z}}^{\prime}(1)$. Alors, le diagramme suivant est commutatif:

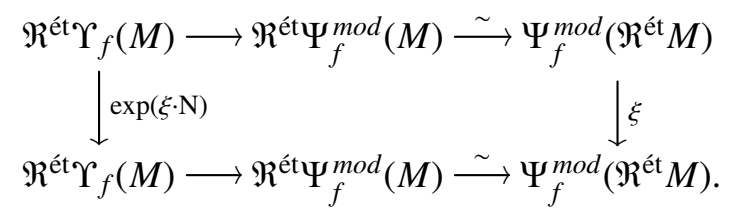

(Bien entendu, $\exp (\xi \cdot \mathrm{N})=\sum_{n=0}^{\infty} n !^{-1}(\xi \cdot \mathrm{N})^{\circ n}$ avec $\mathrm{N}: \Upsilon_{f}(M) \longrightarrow \Upsilon_{f}(M)(-1)$ l'opérateur de monodromie motivique.)

Dans ce qui suit, l'image inverse du motif $\mathscr{K} \in \mathbf{D A}^{\text {ét }}\left(\mathbb{G}_{S}, \Lambda\right)$ suivant $\pi: \eta \longrightarrow \mathbb{G}_{S}$ sera notée abusivement $\mathscr{K}$. On fera de même pour les motifs $\mathscr{L} \operatorname{og}_{n}^{\vee}$ et $\mathscr{L} o g^{\vee}$. Ils seront donc considérés comme objets de $\mathbf{D} \mathbf{A}^{\text {ét }}(\eta, \Lambda \otimes \mathbb{Q})$. D'ailleurs, les anneaux $\Lambda \otimes \mathbb{Q}$ et $\Lambda_{J} \otimes \mathbb{Q}$ seront désignés par $\Pi$ et $\Pi_{J}$ respectivement.

On commence par quelques résultats préliminaires.

Lemme 11.18 - On fixe $n \in \mathbb{N} \sqcup\{\infty\}$ et on convient que $\mathscr{L}_{o g_{\infty}}^{\vee}=\mathscr{L}_{o g}{ }^{\vee}$. On a les propriétés suivantes.

(a) Il existe un isomorphisme canonique $\Upsilon_{S}\left(\mathscr{L} \circ g_{n}^{\vee}\right) \simeq \bigoplus_{i=0}^{n} \Pi_{\sigma}(-i)$ et le morphisme évident $\Upsilon_{S}\left(\mathscr{L}_{\mathrm{og}_{n}}^{\vee}\right) \longrightarrow \Psi_{S}^{\bmod }\left(\mathscr{L}_{\mathrm{og}_{n}}^{\vee}\right)$ est inversible.

(b) Pour tout $S$-schéma quasi-projectif $f: X \longrightarrow S$, les transformations naturelles suivantes sont inversibles :

$-\Upsilon_{f}(-) \otimes \Upsilon_{S}\left(\mathscr{L} \operatorname{og}_{n}^{\vee}\right) \longrightarrow \Upsilon_{f}\left(-\otimes f_{\eta}^{*} \mathscr{L} o g_{n}^{\vee}\right)$, et

$-\Psi_{f}^{\bmod }(-) \otimes \Psi_{S}^{\bmod }\left(\mathscr{L} \operatorname{og}_{n}^{\vee}\right) \longrightarrow \Psi_{f}^{\bmod }\left(-\otimes f_{\eta}^{*} \mathscr{L} \operatorname{og}_{n}^{\vee}\right)$.

(c) Pour tout $S$-schéma quasi-projectif $f: X \longrightarrow S$, on a des isomorphismes canoniques:

$-\Upsilon_{f}\left(-\otimes f_{\eta}^{*} \mathscr{L} \operatorname{og}_{n}^{\vee}\right) \simeq \bigoplus_{i=0}^{n} \Upsilon_{f}(-)(-i)$, et

$-\Psi_{f}^{\text {mod }}\left(-\otimes f_{\eta}^{*} \mathscr{L} 0 g_{n}^{\vee}\right) \simeq \bigoplus_{i=0}^{n} \Psi_{f}^{\text {mod }}(-)(-i)$.

Demonstration La partie (c) s'obtient par conjonction de (a) et (b). On démontre d'abord la partie (b). Puisque $\mathscr{L}_{o g} g_{n}^{\vee}$ appartient à la plus petite sous-catégorie triangulée stable par sommes infinies et contenant les motifs de Tate $\Pi_{\eta}(-i)$ pour $i \in \mathbb{N}$, il suffit de montrer que les transformations naturelles

$$
\Upsilon_{f}(-) \otimes \Upsilon_{S}(\Pi) \longrightarrow \Upsilon_{f}(-) \quad \text { et } \quad \Psi_{f}^{\bmod }(-) \otimes \Psi_{S}^{\bmod }(\Pi) \longrightarrow \Psi_{f}^{\bmod }(-)
$$

sont inversibles. Or $\Upsilon_{S}(\Pi) \simeq \Pi$ et $\Psi_{S}^{\bmod }(\Pi) \simeq \Pi$ d'après les Théorèmes 10.2 et 10.7. Ceci permet de conclure pour (b). Un argument similaire montre que $\Upsilon_{S}\left(\mathscr{L} o g_{n}^{\vee}\right) \longrightarrow \Psi_{S}^{\bmod }\left(\mathscr{L} \operatorname{og}_{n}^{\vee}\right)$ est inversible.

Il reste à calculer $\Upsilon_{S}\left(\mathscr{L} \operatorname{og}_{n}^{\vee}\right)$. Pour cela, on considère le morphisme

$$
\left(\begin{array}{c}
m \\
b \otimes \mathrm{id}
\end{array}\right): \mathscr{K} \otimes \mathscr{L}_{o g} \vee \longrightarrow \mathscr{L}_{o g} \vee \bigoplus \mathscr{L}{ }^{\vee}{ }^{\vee}(-1)
$$

où $m$ est induit par le morphisme canonique $\mathscr{K} \longrightarrow \mathscr{L}_{o g^{\vee}}$ et la multiplication de $\mathscr{L}_{o g^{\vee}}$. D'après [5, Cor. 3.6.8], le morphisme (125) est inversible. Il s'ensuit que $\Upsilon_{S}(\mathscr{K}) \simeq \Upsilon_{S}(\Pi) \bigoplus \Upsilon_{S}(\Pi(-1)) \simeq$ $\Pi \bigoplus \Pi(-1)$. Or, la partie (b) appliquée à $\mathscr{K}$ et une récurrence entraînent que $\Upsilon_{S}\left(\operatorname{Sym}^{n} \mathscr{K}\right) \simeq$ $\operatorname{Sym}^{n} \Upsilon_{S}(\mathscr{K})$. Ceci permet de conclure.

Lemme 11.19 - Soient $f: X \longrightarrow S$ un $S$-schéma quasi-projectif et $M \in \mathbf{D A}^{\mathrm{e}} \mathrm{e}\left(X_{\eta}, \Pi\right)$ un motif constructible. Pour $m \geq 1$ suffisamment grand, le triangle distingué

$$
\chi_{f}\left(M \otimes f_{\eta}^{*} \mathscr{L} o g_{m-1}^{\vee}\right) \longrightarrow \Upsilon_{f}(M) \stackrel{\mathrm{N}_{m}}{\longrightarrow} \Upsilon_{f}(M)(-m) \longrightarrow
$$

déduit de (120), est canoniquement scindé. Autrement dit, il existe un isomorphisme canonique $\chi_{f}\left(M \otimes f_{\eta}^{*} \mathscr{L} \operatorname{og}_{m-1}^{\vee}\right) \simeq \Upsilon_{f}(M) \bigoplus \Upsilon_{f}(M)(-m)[-1]$. 
Demonstration D'après le Théorème 10.9, (ii) et [5, Lem. 3.6.48], on a

$$
\operatorname{hom}_{\mathbf{D A}^{\text {et }}\left(X_{\sigma}, \Lambda\right)}\left(\Upsilon_{f}(M), \Upsilon_{f}(M)(-m)[r]\right)=0 \quad \forall r \in \mathbb{Z}
$$

lorsque $m$ est suffisamment grand. En particulier, $\mathrm{N}_{m}: \Upsilon_{f}(M) \longrightarrow \Upsilon_{f}(M)(-m)$ est nul et le triangle de l'énoncé est scindé. La canonicité de la section $\Upsilon_{f}(M) \longrightarrow \chi_{f}\left(M \otimes f_{\eta}^{*} \mathscr{L}_{o g_{m-1}}^{\vee}\right)$ provient aussi du fait que tout morphisme $\Upsilon_{f}(M) \longrightarrow \Upsilon_{f}(M)(-m)[-1]$ est nul.

Proposition 11.20 - Soient $f: X \longrightarrow S$ un $S$-schéma quasi-projectif et $M \in \mathbf{D A}^{\mathrm{e}}\left(X_{\eta}, \Pi\right)$ un motif constructible. Soit $m \geq 1$ suffisamment grand de sorte que la conclusion du Lemme 11.19 est assurée. Alors, la composition de

$$
\Upsilon_{f}(M) \longrightarrow \chi_{f}\left(M \otimes f_{\eta}^{*} \mathscr{L} o g_{m-1}^{\vee}\right) \longrightarrow \Upsilon_{f}\left(M \otimes f_{\eta}^{*} \mathscr{L} o g_{m-1}^{\vee}\right) \simeq \bigoplus_{i=0}^{m-1} \Upsilon_{f}(M)(-i) \longrightarrow \Upsilon_{f}(M)(-r)
$$

est égale à $\mathrm{N}_{r}$ pour tout $0 \leq r \leq m-1$. (Ci-dessus, la première flèche est donnée par le scindage canonique du Lemme 11.19 et l'avant dernière flèche, celle qui est inversible, est fournie par le Lemme 11.18, (c).)

Demonstration Pour démontrer la proposition, on peut faire tendre $m$ vers l'infini. Autrement dit, il est suffisant de calculer la composition de

$$
\Upsilon_{f}(M) \simeq \chi_{f}\left(M \otimes f_{\eta}^{*} \mathscr{L} o g^{\vee}\right) \longrightarrow \Upsilon_{f}\left(M \otimes f_{\eta}^{*} \mathscr{L} o g^{\vee}\right) \simeq \bigoplus_{i=0}^{\infty} \Upsilon_{f}(M)(-i) \longrightarrow \Upsilon_{f}(M)(-r)
$$

Nous allons d'abord construire un morphisme d'algèbres $\mathscr{L}_{o g^{\vee}} \otimes \mathscr{L} \operatorname{og}^{\vee} \longrightarrow \bigoplus_{i=0}^{\infty} \mathscr{L}_{\operatorname{og}}{ }^{\vee}(-i)$ qui induit l'isomorphisme $\Upsilon_{S}\left(\mathscr{L} \circ g^{\vee}\right) \simeq \bigoplus_{i=0}^{\infty} \Pi(-i)$ du Lemme 11.18. Pour cela, on part du morphisme

$$
\left(\begin{array}{c}
\text { can } \\
\text { can } \circ b
\end{array}\right): \mathscr{K} \longrightarrow \mathscr{L}^{\circ \vee} \bigoplus^{\vee} \mathscr{L}^{\circ}{ }^{\vee}(-1)
$$

(où « can » désigne un morphisme canonique). D'après [5, Cor. 3.6.19], ce morphisme se prolonge d'une manière unique en un morphisme d'algèbres commutatives et unitaires

$$
\vartheta: \mathscr{L}_{o g} \vee \rightarrow \bigoplus_{i=0}^{\infty} \mathscr{L}^{\vee}{ }^{\vee}(-i)
$$

En utilisant l'inclusion évidente $\iota: \mathscr{L}_{o g} \vee \hookrightarrow \bigoplus_{i=0}^{\infty} \mathscr{L}_{o g^{\vee}}(-i)$, on déduit alors un morphisme d'algèbres

$$
\vartheta \cdot \iota: \mathscr{L}_{o g^{\vee}} \otimes \mathscr{L}_{o g} \vee \longrightarrow \bigoplus_{i=0}^{\infty} \mathscr{L} \operatorname{og}^{\vee}(-i)
$$

On peut montrer que ce morphisme est inversible, mais nous n'aurons pas besoin de cela. Remarquons plutôt que le carré

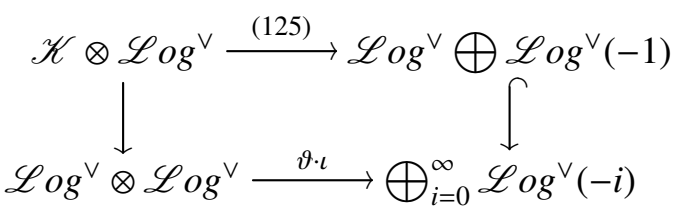

est commutatif. En appliquant $\chi_{S}$, on déduit alors un carré commutatif

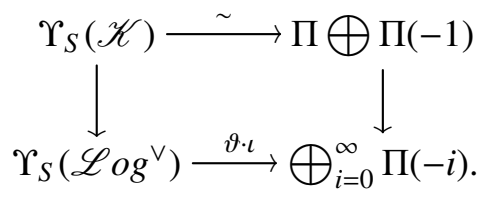


Il en découle que $\vartheta \cdot \iota: \Upsilon_{S}\left(\mathscr{L} \circ g^{\vee}\right) \longrightarrow \bigoplus_{i=0}^{\infty} \Pi(-i)$ coïncide avec l'isomorphisme du Lemme 11.18, (a) (avec $n=\infty$ ). En effet, les deux sont des morphismes d'algèbres qui coïncident sur $\Upsilon_{S}(\mathscr{K})$.

Vue la construction des isomorphismes du Lemme 11.18, (c), on déduit de ce qui précède que la composition de (126) est égale au morphisme obtenu en appliquant $\chi_{f}\left(M \otimes f_{\eta}^{*}(-)\right)$ à la composition de

$$
\mathscr{L} \operatorname{og}^{\vee} \stackrel{\operatorname{id} \otimes u}{\longrightarrow} \mathscr{L}_{o g}^{\vee} \otimes \mathscr{L}_{o g}{ }^{\vartheta} \stackrel{\vartheta \cdot \iota}{\longrightarrow} \bigoplus_{i=0}^{\infty} \mathscr{L} \operatorname{og}^{\vee}(-i) \longrightarrow \mathscr{L}_{o g}{ }^{\vee}(-r) .
$$

(Ci-dessus, on a noté $u$ l'unité de l'algèbre $\mathscr{L}_{o g^{\vee}}{ }^{\vee}$.) Or, la composition de (130) est égale à celle de

$$
\mathscr{L} \operatorname{og}^{\vee} \stackrel{\vartheta}{\longrightarrow} \bigoplus_{i=0}^{\infty} \mathscr{L} \operatorname{og}^{\vee}(-i) \longrightarrow \mathscr{L}_{o g}{ }^{\vee}(-r)
$$

Pour terminer, il reste donc à montrer que la composition de (131) est égale à $\mathrm{N}_{r}$. Ceci fait l'objet du Lemme 11.21 ci-dessous.

LeMme 11.21 - La composition de (131) est égale à $\mathrm{N}_{r}$.

Demonstration Rappelons que $\vartheta: \mathscr{L}_{o g} \vee \longrightarrow \bigoplus_{i=0}^{\infty} \mathscr{L}_{o g^{\vee}}(-i)$ est l'unique morphisme d'algèbres dont la restriction à $\mathscr{K}$ est donnée par (127). Nous allons calculer la composition de

$$
\mathscr{K}^{\otimes n+r} \longrightarrow \mathscr{L} \operatorname{og}_{n+r}^{\vee} \longrightarrow \mathscr{L}_{o g}{ }^{\vee} \stackrel{\vartheta}{\longrightarrow} \bigoplus_{i=0}^{\infty} \mathscr{L} \operatorname{og}^{\vee}(-i) \longrightarrow \mathscr{L}_{o g}^{\vee}(-r)
$$

D'après la construction de $\vartheta$, la composition de (132) est égale à celle de

$$
\mathscr{K}^{\otimes n+r} \longrightarrow \bigoplus_{I \subset \llbracket 1, n+r \rrbracket, \operatorname{card}(I)=n} \bigotimes_{i=1}^{n} \mathscr{L}_{o g^{\vee}}\left(-\delta_{i, I}\right) \longrightarrow \mathscr{L}_{o g^{\vee}}(-r)
$$

avec $\delta_{i, I}=0$ si $i \notin I$ et $\delta_{i, I}=1$ si $i \in I$. La $I$-ième composante de la première flèche ci-dessus est le produit tensoriel des flèches $\mathscr{K} \longrightarrow \mathscr{L} \operatorname{ogg}^{\vee}\left(-\delta_{i, I}\right)$ égales à can si $i \notin I$ et à can $\circ b$ si $i \in I$ (cf. (127)). Puisque l'algèbre $\bigoplus_{i=0}^{\infty} \mathscr{L}_{o g^{\vee}}(-i)$ est commutative, les compositions de

$$
\mathscr{K}^{\otimes n+r} \longrightarrow \bigotimes_{i=1}^{n} \mathscr{L}_{o g^{\vee}}\left(-\delta_{i, I}\right) \longrightarrow \mathscr{L}_{o g^{\vee}}(-r)
$$

sont indépendantes de $I$ et elles sont données par le morphisme évident, à savoir la composition de

$$
\mathscr{K}^{\otimes n+r} \longrightarrow \mathscr{L}_{o g_{n+r}^{\vee}} \stackrel{b^{r}}{\longrightarrow} \mathscr{L}_{o g_{n}^{\vee}}^{\vee}(-r) \longrightarrow \mathscr{L}^{\circ g^{\vee}}(-r) .
$$

Ainsi, la composition de (132) est égale à celle de

$$
\mathscr{K}^{\otimes n+r} \longrightarrow \mathscr{L} \operatorname{og}_{n+r}^{\vee} \stackrel{C_{n+r}^{n} \cdot b^{r}}{\longrightarrow} \mathscr{L} \operatorname{og}_{n}^{\vee}(-r) \longrightarrow \mathscr{L}_{o g^{\vee}}(-r)
$$

Le résultat recherché découle maintenant de la construction des morphismes $\mathrm{N}_{r}$.

Les deux lemmes qui suivent décrivent l'action par monodromie sur les cycles proches modérés dans des cas simples.

Lemme 11.22 - Il existe un isomorphisme $\Psi_{S}^{\text {mod }}\left(\Re^{\text {ét }}(\mathscr{K})\right) \simeq \Lambda_{J} \oplus \Lambda_{J}(-1)$ modulo lequel $\xi \in \widehat{\mathbb{Z}}^{\prime}(1)$ agit par la matrice

$$
\left(\begin{array}{cc}
1 & \xi^{-1} \\
0 & 1
\end{array}\right)
$$

Demonstration Il suffit de prouver le résultat analogue pour les coefficients finis. Ainsi, nous travaillerons dans $\mathbf{D}^{\text {ét }}\left(-, \mathbb{Z} / \ell^{n}\right)$ avec $n \in \mathbb{N}$ et $\ell$ un nombre premier différent de $p$. La réalisation étale de l'extension de Kummer est un faisceau de $\mathbb{Z} / \ell^{n}$-modules sur Et $/ \eta$ qui s'inscrit dans une suite exacte courte

$$
0 \longrightarrow \mathbb{Z} / \ell^{n} \longrightarrow \mathfrak{R}^{\text {ét }} \mathscr{K} \longrightarrow \mathbb{Z} / \ell^{n}(-1) \longrightarrow 0 .
$$


L'extension ci-dessus est determinée par l'image de $\left(-e_{\mathrm{K}}[1]\right) \in \operatorname{hom}_{\mathbf{D A}^{\mathrm{et}}\left(\eta, \mathbb{Z}_{(\ell)}\right)}\left(\mathbb{Z}_{(\ell)}(-1), \mathbb{Z}_{(\ell)}[1]\right)$ dans le groupe $\operatorname{ext}^{1}\left(\mathbb{Z} / \ell^{n}(-1), \mathbb{Z} / \ell^{n}\right)$. Or, on a un diagramme commutatif

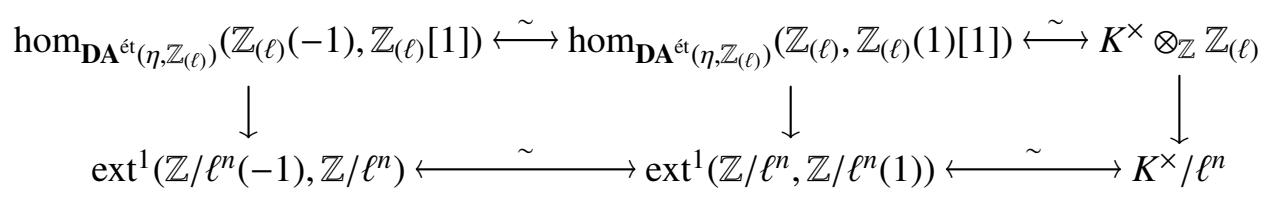

et $\left(-e_{K}[1]\right)$ correspond à $\pi^{-1} \otimes 1 \in K^{\times} \otimes_{\mathbb{Z}} \mathbb{Z}_{(\ell)}$ par les identifications de la ligne horizontale supérieure. (Rappelons que $\pi \in K^{\times}$est l'uniformisante.) Il s'ensuit que (135) est isomorphe à l'extension

$$
0 \longrightarrow \mu_{\ell^{n}}(-1) \longrightarrow \mathcal{F}(-1) \longrightarrow \mathbb{Z} / \ell^{n}(-1) \longrightarrow 0,
$$

avec $\mathcal{F}$ le faisceau étale associé au préfaisceau qui envoie le spectre d'une $K$-algèbre étale $L$ sur le groupe

$$
\left\{a \in L^{\times} \mid a^{\ell^{n}} \in \pi^{\mathbb{Z}}\right\} / \pi^{\mathbb{Z}} .
$$

(Ci-dessus, on a noté $\pi^{\mathbb{Z}}$ le sous-groupe $\left\{\pi^{r} \mid r \in \mathbb{Z}\right\} \subset L^{\times}$.) Par construction, on a un isomorphisme canonique $\mathcal{F}(\widetilde{\eta}) \simeq \mu_{\ell^{n}}(\widetilde{\eta}) \bigoplus \mathbb{Z} / \ell^{n} \cdot \pi^{-1 / \ell^{n}}$ et l'action de $\xi \in \widehat{\mathbb{Z}}^{\prime}(1)$ est donnée par la matrice de l'énoncé. On en déduit aussitôt un isomorphisme $\Psi_{S}^{\bmod }(\mathcal{F}) \simeq \mathbb{Z} / \ell^{n}(1) \oplus \mathbb{Z} / \ell^{n}$ et il est facile de voir que la composition de (124) est encore donnée par la matrice de l'énoncé. Ceci démontre le lemme.

Corollaire 11.23 - Pour $n \in \mathbb{N}$, le Lemme 11.18, (a) et l'isomorphisme de commutation $\Psi_{S}^{\text {mod }} \Re^{\text {ét }}\left(\mathscr{L} \operatorname{og}_{n}^{\vee}\right) \simeq \Re^{\text {ét }} \Psi_{S}^{\bmod }\left(\mathscr{L}\right.$ og $\left.g_{n}^{\vee}\right)$ fournissent un isomorphisme canonique :

$$
\Psi_{S}^{\text {mod }}\left(\Re^{\mathrm{e} t} \mathscr{L} \operatorname{og}_{n}^{\vee}\right) \simeq \bigoplus_{i=0}^{n} \Pi_{J}(-i) .
$$

Modulo cet isomorphisme, $\xi \in \hat{\mathbb{Z}}^{\prime}(1)$ agit par la matrice triangulaire supérieure

$$
\mathrm{L}_{n}(\xi)=\left(\begin{array}{cccccc}
1 & \xi^{-1} & \xi^{-2} & \xi^{-3} & \ldots & \xi^{-n} \\
0 & 1 & 2 \cdot \xi^{-1} & 3 \cdot \xi^{-2} & \ldots & n \cdot \xi^{-n+1} \\
& 0 & 1 & 3 \cdot \xi^{-1} & \ldots & \frac{n(n-1)}{2} \cdot \xi^{-n+2} \\
& & \ddots & 1 & & \vdots \\
& & & & \ddots & n \cdot \xi^{-1} \\
& & & & & 1
\end{array}\right)
$$

où la $(i, j)$-ième entrée, avec $0 \leq j \leq i \leq n$, est donnée $\operatorname{par} C_{i}^{j} \cdot \xi^{-i+j}$.

Demonstration Lorsque $n=0$, il n'y a rien à montrer. Lorsque $n=1$, le résultat découle du Lemme 11.22. Toutefois, nous devons faire attention au problème suivant. Nous avons deux isomorphismes $\Psi_{S}^{\bmod } \mathfrak{R}^{\text {ét }}(\mathscr{K}) \simeq \Pi_{J} \bigoplus \Pi_{J}(-1)$, le premier est déduit du Lemme 11.18, (a) et le second est celui construit dans la preuve du Lemme 11.22 par passage à la limite et extension des scalaires à $\Lambda_{J}$. Or, il n'est pas clair que ces deux isomorphismes sont les mêmes. Voici comment on peut s'en sortir. En composant l'inverse du premier isomorphisme avec le second isomorphisme, on obtient un automorphisme $\theta$ de $\Pi_{J} \bigoplus \Pi_{J}(-1)$ et il suffira de montrer que $\theta$ commute à la matrice du Lemme 11.22. Or, il est facile de voir qu'on a un diagramme commutatif

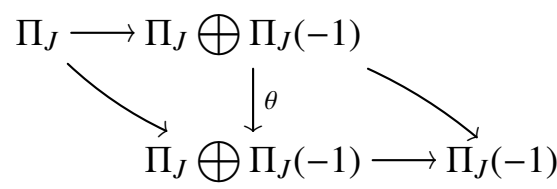

où les flèches horizontales et obliques sont des inclusions et des projections évidentes. Ainsi, $\theta$ est donné par une matrice triangulaire supérieure avec des 1 sur la diagonale. En particulier, elle commute avec la matrice du Lemme 11.22. 
Enfin, pour le cas général, on remarque que l'isomorphisme (136) est donné par la composition des isomorphismes $\widehat{\mathbb{Z}^{\prime}}(1)$-equivariants suivants :

$$
\Psi_{S}^{\text {mod }} \mathfrak{R}^{\mathrm{é}}\left(\operatorname{Sym}^{n} \mathscr{K}\right) \simeq \operatorname{Sym}^{n} \Psi_{S}^{\text {mod }} \mathfrak{R}^{\mathrm{ét}}(\mathscr{K}) \simeq \operatorname{Sym}^{n}(\Pi \bigoplus \Pi(-1)) .
$$

Ceci permet de conclure.

On peut maintenant donner la preuve du Théorème 11.17.

Demonstration Soit $m \geq 1$ un entier suffisamment grand de sorte que la conclusion du Lemme 11.19 est vérifiée. Le morphisme canonique :

$$
\chi_{f} \Re^{\text {ét }}\left(M \otimes f_{\eta}^{*} \mathscr{L} o g_{m-1}^{\vee}\right) \longrightarrow \Psi_{f}^{\bmod } \Re^{\text {ét }}\left(M \otimes f_{\eta}^{*} \mathscr{L} \circ g_{m-1}^{\vee}\right)
$$

est $\widehat{\mathbb{Z}}^{\prime}(1)$-équivariant lorsqu'on fait agir $\widehat{\mathbb{Z}}^{\prime}(1)$ par l'identité sur la source et par monodromie sur le but. Par ailleurs, on a un isomorphisme canonique de $\widehat{\mathbb{Z}^{\prime}}(1)$-représentations :

$$
\Psi_{f}^{\text {mod }} \Re^{\text {ét }}\left(M \otimes f_{\eta}^{*} \mathscr{L} o g_{m-1}^{\vee}\right) \simeq\left(\Psi_{f}^{\text {mod }} \mathfrak{R}^{\text {et }}(M)\right) \otimes_{\Pi_{J}}\left(\bigoplus_{i=0}^{m-1} \Pi_{J}(-i)\right) .
$$

Il est construit comme dans la preuve du Lemme 11.18. D'autre part, on a des isomorphismes canoniques (cf. le Lemme 11.19) :

$$
\mathfrak{R}^{\mathrm{ét}} \Upsilon_{f}(M) \oplus \mathfrak{R}^{\mathrm{ét}} \Upsilon_{f}(M)(-m)[-1] \simeq \mathfrak{R}^{\mathrm{e} t} \chi_{f}\left(M \otimes f_{\eta}^{*} \mathscr{L} o g_{m-1}^{\vee}\right) \simeq \chi_{f} \mathfrak{R}^{\mathrm{ét}}\left(M \otimes f_{\eta}^{*} \mathscr{L} o g_{m-1}^{\vee}\right) .
$$

On en déduit alors un morphisme $\widehat{\mathbb{Z}}^{\prime}(1)$-équivariant

$$
\Re^{\text {ét }} \Upsilon_{f}(M) \longrightarrow\left(\Psi_{f}^{\text {mod }} \Re^{\text {ét }}(M)\right) \otimes_{\Pi_{J}}\left(\bigoplus_{i=0}^{m-1} \Pi_{J}(-i)\right) .
$$

Le groupe $\widehat{\mathbb{Z}}^{\prime}(1)$ agit par l'identité sur $\Re^{\text {ét }} \Upsilon_{f}(M)$, par monodromie sur $\Psi_{f}^{\text {mod }} \Re^{\text {ét }}(M)$ et via les matrices $\mathrm{L}_{m-1}(-)$ du Corollaire 11.23 sur $\bigoplus_{i=0}^{m-1} \Pi_{J}(-i)$.

Pour $0 \leq r \leq m-1$, on note $\varphi_{r}: \Re^{\text {ét }} \Upsilon_{f}(M) \longrightarrow \Psi_{f}^{\text {mod }} \mathfrak{R}^{\text {ét }}(M)(-r)$ la $r$-ième composante de (140). D'après les constructions, $\varphi_{r}$ est égale à la composition de

$$
\begin{aligned}
& \mathfrak{R}^{\mathrm{e} t} \Upsilon_{f}(M) \longrightarrow \mathfrak{R}^{\mathrm{e} e} \chi_{f}\left(M \otimes f^{*} \mathscr{L}_{o g_{m-1}}^{\vee}\right) \longrightarrow \mathfrak{R}^{\mathrm{e} t} \Upsilon_{f}\left(M \otimes f^{*} \mathscr{L} \circ g_{m-1}^{\vee}\right) \\
& \simeq \bigoplus_{i=0}^{m-1} \Re^{\text {ét }} \Upsilon_{f}(M)(-i) \longrightarrow \Re^{\text {ét }} \Psi_{f}^{\bmod }(M)(-r) \simeq \Psi_{f}^{\text {mod }} \Re^{\text {ét }}(M)(-r) \text {. }
\end{aligned}
$$

D'après la Proposition 11.20, cette composition est égale à

$$
\mathfrak{R}^{\text {ét }} \Upsilon_{f}(M) \stackrel{\mathrm{N}_{r}}{\longrightarrow} \Re^{\text {ét }} \Upsilon_{f}(M)(-r) \longrightarrow \mathfrak{R}^{\text {ét }} \Psi_{f}^{\bmod }(M)(-r) \simeq \Psi_{f}^{\bmod } \mathfrak{R}^{\text {ét }}(M)(-r) .
$$

Ainsi, en notant can : $\mathfrak{R}^{\mathrm{ét}} \Upsilon_{f}(M) \longrightarrow \Psi_{f}^{\text {mod }} \mathfrak{R}^{\mathrm{e}}(M)$ la composition de la seconde et troisième flèches ci-dessus, on a la formule $: \varphi_{r}=$ can $\circ \mathrm{N}_{r}$.

Fixons $\xi \in \widehat{\mathbb{Z}}^{\prime}(1)$ et notons $\mathrm{T}(\xi)$ l'automorphisme de $\Psi_{f}^{\text {mod }} \mathfrak{R}^{\text {ét }}(M)$ égal à l'action par monodromie de $\xi$. Puisque (140) est équivariant, les deux compositions suivantes sont égales :

$$
\begin{gathered}
\mathfrak{R}^{\text {ét }} \Upsilon_{f}(M) \longrightarrow \bigoplus_{i=0}^{m-1} \Psi_{f}^{m o d} \Re^{\text {ét }}(M)(-i) \longrightarrow \Psi_{f}^{m o d} \Re^{\text {ét }}(M) \\
\mathfrak{R}^{\text {ét }} \Upsilon_{f}(M) \longrightarrow \bigoplus_{i=0}^{m-1} \Psi_{f}^{m o d} \Re^{\text {ét }}(M)(-i) \stackrel{\mathrm{T}(\xi) \otimes \mathrm{L}_{m-1}(\xi)}{\longrightarrow} \bigoplus_{i=0}^{m-1} \Psi_{f}^{\text {mod }} \mathfrak{R}^{\text {ét }}(M)(-i) \longrightarrow \Psi_{f}^{\text {mod }} \mathfrak{R}^{\text {ét }}(M) .
\end{gathered}
$$

(Ci-dessus, les deux dernières flèches sont les projections sur les 0-ièmes facteurs.) Un calcul facile donne alors la relation : can $=\sum_{r=0}^{m-1}\left(\mathrm{~T}(\xi) \otimes \xi^{-r}\right) \circ \varphi_{r}$. Vu le calcul précédant, on a :

$$
\begin{aligned}
\left(\mathrm{T}(\xi) \otimes \xi^{-r}\right) \circ \varphi_{r} & =\left(\mathrm{T}(\xi) \otimes \xi^{-r}\right) \circ \text { can } \circ \mathrm{N}_{r} \\
& =\mathrm{T}(\xi) \circ \text { can } \circ\left(\xi^{-r} \cdot \mathrm{N}_{r}\right) .
\end{aligned}
$$


On trouve alors la relation can $=\mathrm{T}(\xi) \circ$ can $\circ \sum_{i=0}^{m-1} \xi^{-r} \cdot \mathrm{N}_{r}$. En utilisant le Lemme 11.10 et en multipliant à gauche par l'inverse de $\mathrm{T}(\xi)$, on obtient en fin de compte la relation $\mathrm{T}(\xi)^{-1} \circ$ can $=$ can $\circ \exp \left(\xi^{-1} \cdot \mathrm{N}\right)$. Puisque $\exp \left(\xi^{-1} \cdot \mathrm{N}\right)$ est l'inverse de $\exp (\xi \cdot \mathrm{N})$, le théorème est démontré.

\section{Annexe A}

\section{Changement de coefficients}

Soit $\mathfrak{M}$ une catégorie de modèles monoïdale, symétrique et unitaire (voir par exemple [5, Déf. 4.1.57]), munie d'un ensemble d'objets $\mathcal{E} \subset \mathfrak{M}$. On suppose que les conditions suivantes sont satisfaites.

1. M est propre à gauche, présentable par cofibrations et stable.

2. Les équivalences faibles et les fibrations de $\mathfrak{M}$ sont stables par coproduits et colimites filtrantes.

3. Les objets de $\varepsilon$ sont cofibrants, homotopiquement compacts et engendrent la catégorie triangulée avec sommes infinies $\mathbf{H o}(\mathfrak{M})$. De plus, $\mathcal{E}$ est stable (à isomorphisme près dans $\mathbf{H o}(\mathcal{E})$ ) par les foncteurs de suspension et cosuspension.

Une telle catégorie $\mathfrak{M}$ sera appelée une catégorie de coefficients. Il s'agit d'une notion plus restrictive que celle adoptée dans [4, Déf. 4.4.23] auquel nous renvoyons le lecteur pour la signification des termes employés. Le choix de l'ensemble $\mathcal{E}$ importe peu; on peut toujours supposer que $\mathcal{E}$ est un système de représentants des classes d'isomorphismes dans $\mathbf{H o}(\mathfrak{M})$ des objets compacts.

On fixe un schéma $S$ et le choix d'une topologie $\tau \in\{\mathrm{Nis}$, ét $\}$ sur $\mathrm{Sm} / S$. Étant donnée une catégorie de coefficients $\mathfrak{M}$, on dispose de deux catégories de $S$-motifs à coefficients dans $\mathfrak{M}$. La première, appelée la catégorie effective, est donnée par :

$$
\mathbf{S H}_{\mathfrak{M}}^{\mathrm{eff}, \tau}(S)=\mathbf{H o}_{\mathbb{A}^{1}-\tau}(\mathbf{P S h}(\operatorname{Sm} / S, \mathfrak{M})) .
$$

C'est la catégorie homotopique, relativement à la structure $\left(\mathbb{A}^{1}, \tau\right)$-locale [5, Déf. 4.5.12], de la catégorie des préfaisceaux sur $\mathrm{Sm} / S$ à valeurs dans $\mathfrak{M}$. La seconde, qualifiée de stable, est donnée par :

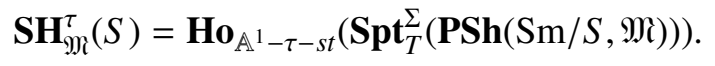

C'est la catégorie homotopique, relativement à la structure $\left(\mathbb{A}^{1}, \tau\right)$-locale stable [5, Déf. 4.5.21], de la catégorie des $T$-spectres symétriques en préfaisceaux sur $\mathrm{Sm} / S$ à valeurs dans $\mathfrak{M}$. (Ici $T=T_{S}$ est un remplacement projectivement cofibrant du préfaisceau quotient $\left(\mathbb{P}_{S}^{1} \otimes \mathbb{1}\right) /\left(\infty_{S} \otimes \mathbb{1}\right)$.)

Lorsque $\mathfrak{M}=\operatorname{Compl}(\Lambda)$, avec $\Lambda$ un anneau commutatif, on retrouve les catégories $\mathbf{D A}^{\text {eff, } \tau}(S, \Lambda)$ et $\mathbf{D A}^{\tau}(S, \Lambda)$ auxquelles nous nous sommes intéressés dans cet article. Lorsque $\tau=$ Nis, on omettra la mention de la topologie et on notera simplement $\mathbf{S H}_{\mathfrak{M}}^{\mathrm{eff}}(S), \mathbf{S H}_{\mathfrak{M}}(S), \mathbf{D A}^{\mathrm{eff}}(S, \Lambda)$ et $\mathbf{D A}(S, \Lambda)$ ces catégories.

Dans cette appendice, on étudie la dépendance des catégories des $S$-motifs vis à vis de la catégorie des coefficients $\mathfrak{M}$. Ainsi, on se donne une deuxième catégorie de coefficients $\mathfrak{N}$ et un foncteur de Quillen à gauche $a: \mathfrak{M} \longrightarrow \mathfrak{N}$, monoïdal symétrique et unitaire. On note $b$ son adjoint à droite. Sauf mention explicite du contraire, on prendra pour remplacement projectivement cofibrant de $\left(\mathbb{P}_{S}^{1} \otimes \mathbb{1}\right) /\left(\infty_{S} \otimes \mathbb{1}\right) \in \mathbf{P S h}(\operatorname{Sm} / S, \mathfrak{R})$ le préfaisceau $a\left(T_{S}\right)$ que l'on notera simplement $T_{S}$ ou même $T$. On commence par un résultat général.

Lemme A.1 - Soit (S, top) un site et supposons qu'il admet suffisamment de points. Alors, le foncteur a $: \mathbf{P S h}(\mathcal{S}, \mathfrak{M}) \longrightarrow \mathbf{P S h}(\mathcal{S}, \mathfrak{N})$ est de Quillen à gauche relativement aux structures injectives (resp. projectives) top-locales (cf. [5, Déf. 4.4.33]).

Demonstration Il est clair que $a: \operatorname{PSh}(\mathcal{S}, \mathfrak{M}) \longrightarrow \operatorname{PSh}(\mathcal{S}, \mathfrak{N})$ est de Quillen à gauche relativement aux structures injectives (resp. projectives) non localisées. Il suffit donc de prouver que ce foncteur préserve les équivalences top-locales entre préfaisceaux injectivement cofibrants. 
Soit $f: F \longrightarrow G$ une équivalence top-locale entre préfaisceaux injectivement cofibrants sur $\mathcal{S}$ à valeurs dans $\mathfrak{M}$. On vérifie que le morphisme $a(f)$ est une équivalence top-locale en montrant qu'il induit des équivalences faibles (dans $\mathfrak{N}$ ) sur les fibres (cf. la preuve de [5, Prop. 4.4.62]).

Soit $x$ un point du site $(\mathcal{S}, t o p)$. On cherche à prouver que

$$
\operatorname{colim}_{U \in \mathcal{S}, e \in x^{*}(U)} a(F(U)) \longrightarrow \operatorname{colim}_{U \in \mathcal{S}, e \in x^{*}(U)} a(G(U))
$$

est une équivalence faible dans $\mathfrak{N}$. Or, les colimites filtrantes préservent les équivalences faibles dans $\mathfrak{R}$. Il vient que les colimites ci-dessus, sont des colimites homotopiques. Par ailleurs, les colimites homotopiques commutent avec La. On peut donc réécrire le morphisme (143) dans $\mathbf{H o}(\mathfrak{M})$ de la manière suivante :

$$
\mathrm{L} a\left(\operatorname{colim}_{U \in \mathcal{S}, e \in x^{*}(U)} F(U)\right) \longrightarrow \mathrm{L} a\left(\operatorname{colim}_{U \in \mathcal{S}, e \in x^{*}(U)} G(U)\right) .
$$

Ceci prouve le résultat recherché.

\section{Proposition A.2 -}

(a) Le foncteur

$$
a: \operatorname{PSh}(\mathrm{Sm} / S, \mathfrak{M}) \longrightarrow \operatorname{PSh}(\mathrm{Sm} / S, \mathfrak{N})
$$

est de Quillen à gauche relativement aux structures projectives $\left(\mathbb{A}^{1}, \tau\right)$-locales.

(b) Le foncteur

$$
a: \operatorname{Spt}_{T}^{\Sigma}(\mathbf{P S h}(\operatorname{Sm} / S, \mathfrak{M})) \longrightarrow \operatorname{Spt}_{T}^{\Sigma}(\mathbf{P S h}(\operatorname{Sm} / S, \mathfrak{N}))
$$

est de Quillen à gauche relativement aux structures projectives $\left(\mathbb{A}^{1}, \tau\right)$-locales stables.

Demonstration Pour voir que (144) est de Quillen à gauche, relativement aux structures $\left(\mathbb{A}^{1}, \tau\right)$ locales, on utilise le Lemme A.1, et le fait qu'il envoie la flèche $\mathbb{A}_{U}^{1} \otimes A \longrightarrow U \otimes A$ sur la flèche $\mathbb{A}_{U}^{1} \otimes a(A) \longrightarrow U \otimes a(A)$ pour tout $U \in \mathrm{Sm} / S$ et $A \in \mathfrak{M}$. De même, pour montrer que (145) est de Quillen à gauche, relativement aux structures $\left(\mathbb{A}^{1}, \tau\right)$-locales stables, on utilise que ce dernier envoie la flèche $\omega_{F}^{n}: \operatorname{Sus}_{T, \Sigma}^{n+1}\left(T_{S} \otimes F\right) \longrightarrow \operatorname{Sus}_{T, \Sigma}^{n}(F)$ (cf. [5, page 239, (82)]) sur la flèche $\omega_{a(F)}^{n}$ : $\operatorname{Sus}_{T, \Sigma}^{n+1}\left(T_{S} \otimes a(F)\right) \longrightarrow \operatorname{Sus}_{T, \Sigma}^{n}(a(F))$ pour tout $n \in \mathbb{N}$ et $F \in \mathbf{P S h}(\operatorname{Sm} / S, \mathfrak{M})$.

D'après la Proposition A.2, on dispose donc de deux adjonctions

$$
(\mathrm{L} a, \mathrm{R} b): \mathbf{S H}_{\mathfrak{M}}^{\mathrm{eff}, \tau}(S) \longrightarrow \mathbf{S H}_{\mathfrak{M}}^{\mathrm{eff}, \tau}(S) \quad \text { et } \quad(\mathrm{L} a, \mathrm{R} b): \mathbf{S H}_{\mathfrak{M}}^{\tau}(S) \longrightarrow \mathbf{S H}_{\mathfrak{M}}^{\tau}(S) .
$$

De plus, les foncteurs $L a$ sont monoïdaux, symétriques et unitaires. Notons aussi le fait suivant.

Lemme A.3 - Soit $f: T \longrightarrow S$ un morphisme de schémas.

1. Alors, les carrés suivants
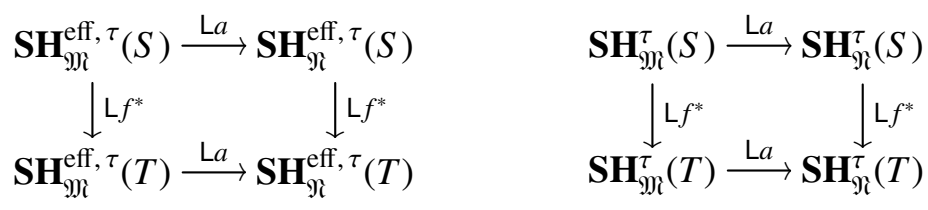

commutent à des isomorphismes (de foncteurs monoüdaux, symétriques et unitaires) canoniques près.

2. Supposons que f est lisse. Alors, les carrés suivants
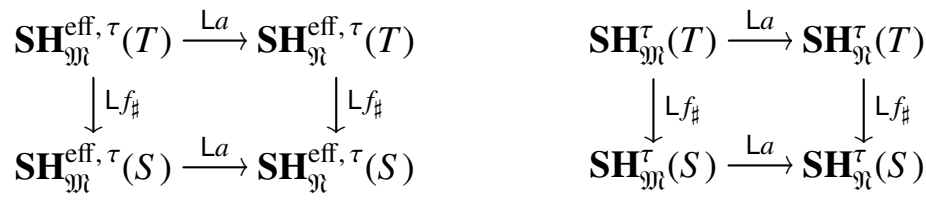

commutent à des isomorphismes canoniques près. 
Démonstration. - Les foncteurs $a, f^{*}$ et $f_{\sharp}$ de l'énoncé sont tous de Quillen à gauche. Il suffit donc de vérfier les compatibilités recherchées au niveau des catégories de modèles où elles sont trivialement satisfaites.

Lemme A.4 - On suppose que $b: \mathfrak{N} \longrightarrow \mathfrak{M}$ préserve les équivalences faibles et qu'il commute aux colimites arbitraires. Alors, le foncteur

$$
b: \operatorname{Spt}_{T}^{\Sigma}(\mathbf{P S h}(\operatorname{Sm} / S, \mathfrak{N})) \longrightarrow \operatorname{Spt}_{T}^{\Sigma}(\mathbf{P S h}(\operatorname{Sm} / S, \mathfrak{M}))
$$

préserve les équivalences $\left(\mathbb{A}^{1}, \tau\right)$-locales stables. L'énoncé analogue est vrai pour les catégories effectives.

Demonstration Montrons d'abord que le foncteur $b: \operatorname{PSh}(\operatorname{Sm} / S, \mathfrak{N}) \longrightarrow \mathbf{P S h}(\operatorname{Sm} / S, \mathfrak{M})$ préserve les équivalences $\tau$-locales. Soit $f: F \longrightarrow K$ une équivalence $\tau$-locale entre préfaisceaux sur $\mathrm{Sm} / S$ à valeurs dans $\mathfrak{N}$. Pour montrer que $b(f)$ est une équivalence $\tau$-locale, il suffit de montrer que $x^{*}(b(f))$ est une équivalence faible dans $\mathfrak{M}$ pour tout point du site $(\mathrm{Sm} / S, \tau)$. Vu que $b$ commute aux colimites, et en particulier au colimites filtrantes, on obtient que $x^{*}(b(f))=b\left(x^{*}(f)\right)$. Or, $b$ préserve les équivalences faibles. Ceci entraîne le résultat recherché.

Montrons maintenant que le foncteur $b: \operatorname{PSh}(\operatorname{Sm} / S, \mathfrak{N}) \longrightarrow \mathbf{P S h}(\operatorname{Sm} / S, \mathfrak{M})$ préserve les équivalences $\left(\mathbb{A}^{1}, \tau\right)$-locales. Pour cela, on utilise un résultat général sur les localisations de Bousfield, à savoir [5, Prop. 4.2.74]. Dans le cas qui nous intéresse, ce résultat affirme que la classe $\mathbf{W}_{\mathbb{A}^{1}-\tau}$ des équivalences $\left(\mathbb{A}^{1}, \tau\right)$-locales est la plus petite classe $\mathcal{C}$ de flèches vérifiant les trois conditions suivantes :

- les équivalences $\tau$-locales sont dans $\mathcal{C}$ ainsi que les flèches de la forme $\mathbb{A}_{U}^{1} \otimes B \longrightarrow U \otimes B$ pour $U \in \mathrm{Sm} / S$ et $B \in \mathfrak{N}$;

- C vérifie la propriété 2 de 3 ;

- les flèches de $\mathcal{C}$ qui sont aussi des cofibrations projectives forment une classe qui est stable par « push-out» et par composition transfinie.

Vu que $b\left(\mathbb{A}_{U}^{1} \otimes B \longrightarrow U \otimes B\right)$ s'identifie à $\mathbb{A}_{U}^{1} \otimes b(B) \longrightarrow U \otimes b(B)$ qui est une équivalence ( $\mathbb{A}^{1}$, ét)locale, il est suffisant de prouver le résultat suivant. Soient $f: F \longrightarrow G$ et $g: F \longrightarrow F^{\prime}$ deux flèches dans $\mathbf{P S h}(\operatorname{Sm} / S, \mathfrak{N})$, et supposons que $f$ est une cofibration projective et que $b(f)$ est une équivalence $\left(\mathbb{A}^{1}, \tau\right)$-locale. Alors, le « push-out » de $b(f)$ par $b(g)$ est encore une équivalence $\left(\mathbb{A}^{1}, \tau\right)$-locale. Pour prouver cette propriété, formons un carré cocartésien de préfaisceaux :

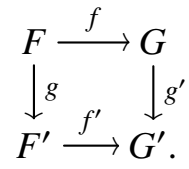

Puisque $\mathfrak{R}$ est stable, ce carré est homotopiquement cartésien relativement à la structure projective non localisée. Puisque le foncteur $b: \mathfrak{N} \longrightarrow \mathfrak{M}$ est de Quillen à droite et qu'il préserve les équivalences faibles, le carré

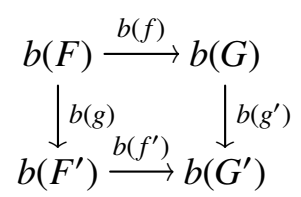

est encore homotopiquement cartésien relativement à la structure non localisée. En utilisant que $\mathfrak{M}$ est stable, on déduit qu'il est aussi homotopiquement cocartésien. Or, les équivalences ( $\mathbb{A}^{1}$, ét)locales sont stables par «push-out » homotopique. Ceci prouve que $b\left(f^{\prime}\right)$ est bien une équivalence $\left(\mathbb{A}^{1}\right.$, ét)-locale.

Il reste à montrer que le foncteur (147) préserve les équivalences $\left(\mathbb{A}^{1}, \tau\right)$-locales stables sachant qu'il préserve les équivalences $\left(\mathbb{A}^{1}, \tau\right)$-locales niveau par niveau. Pour cela, on utilise une deuxième fois [5, Prop. 4.2.74] qu'on applique à la localisation de Bousfield responsable du passage de la structure $\left(\mathbb{A}^{1}, \tau\right)$-locale niveau par niveau à la structure $\left(\mathbb{A}^{1}, \tau\right)$-locale stable. L'argument 
est très similaire à celui qu'on a utilisé ci-dessus pour traiter le cas des équivalences $\left(\mathbb{A}^{1}, \tau\right)$-locales. Les détails sont laissés au lecteur.

Corollaire A.5 - On suppose que $b: \mathfrak{N} \longrightarrow \mathfrak{M}$ préserve les équivalences faibles et qu'il commute aux colimites arbitraires. Soit $f: T \longrightarrow S$ un morphisme de schémas. Alors, les faces carrées suivantes
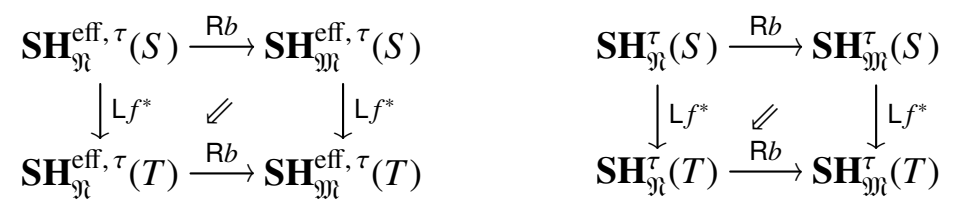

sont inversibles.

Demonstration On traite uniquement le cas stable. Les foncteurs $\mathrm{L} f^{*}$ commutent aux sommes infinies. Il en est de même des foncteurs $\mathrm{R} b$ comme il découle aussitôt du Lemme A.4. Il suffit donc de prouver que la transformation naturelle $\mathrm{L} f^{*} \circ \mathrm{R} b \longrightarrow \mathrm{R} b \circ \mathrm{L} f^{*}$ est inversible après application sur les objets de la forme $\operatorname{Sus}_{T, \Sigma}^{n}(U \otimes B)$ avec $n \in \mathbb{N}, U \in \operatorname{Sm} / S$ et $B \in \mathfrak{N}$. En utilisant encore une fois le Lemme A.4, on trouve que $\mathrm{L} f^{*} \circ \mathrm{R} b\left(\operatorname{Sus}_{T, \Sigma}^{n}(U \otimes B)\right)$ et $\mathrm{R} b \circ \mathrm{L} f^{*}\left(\operatorname{Sus}_{T, \Sigma}^{n}(U \otimes B)\right)$ sont canoniquement isomorphes à $\operatorname{Sus}_{T, \Sigma}^{n}(U \otimes b(B))$.

Les deux résultats suivants sont faciles mais bien utiles.

Lemme A.6 - Si le foncteur $\mathrm{R} b: \mathbf{H o}(\mathfrak{M}) \longrightarrow \mathbf{H o}(\mathfrak{M})$ est conservatif, il en est de même $d u$ foncteur $\mathrm{R} b: \mathbf{S H}_{\mathfrak{M}}^{\tau}(S) \longrightarrow \mathbf{S H}_{\mathfrak{M}}^{\tau}(S)$. (Le même énoncé est vrai pour les catégories effectives.)

Demonstration Soit $f: \mathbf{E} \longrightarrow \mathbf{F}$ un morphisme de $T$-spectres symétriques en préfaisceaux sur $\mathrm{Sm} / S$ à valeurs dans $\mathfrak{N}$. On suppose que $\mathrm{R} b(f)$ est inversible dans $\mathbf{S H}_{\mathfrak{M}}^{\tau}(S)$. Il faut montrer que $f$ est inversible dans $\mathbf{S H}_{\mathfrak{N}}^{\tau}(S)$. Pour cela, on ne restreint pas la généralité en supposant que $\mathbf{E}$ et $\mathbf{F}$ sont stablement projectivement $\left(\mathbb{A}^{1}, \tau\right)$-fibrants. Alors, $b(\mathbf{E})$ et $b(\mathbf{F})$ sont encore stablement projectivement $\left(\mathbb{A}^{1}, \tau\right)$-fibrants. Il vient aussitôt que $b(f)$ est une équivalence faible de préfaisceaux niveau par niveau. Puisque $\mathrm{R} b$ est conservatif, il vient que $f$ est aussi une équivalence faible de préfaisceaux niveau par niveau. Ceci termine la preuve du lemme.

Lemme A.7 - Supposons que a $: \mathfrak{M} \longrightarrow \mathfrak{N}$ est une équivalence de Quillen à gauche. Alors, il en est de même des foncteurs (144) et (145).

Demonstration On traite uniquement le cas stable. On montre que les morphismes d'unité et de counité de l'adjonction ( $\mathrm{L} a, \mathrm{R} b)$ sont inversibles. Soit $\mathbf{E}$ un $T$-spectre symétrique en préfaisceaux sur $\mathrm{Sm} / S$ à valeurs dans $\mathfrak{M}$. On cherche à montrer que $\mathbf{E} \longrightarrow \mathrm{R} b \circ \mathrm{L} a(\mathbf{E})$ est inversible. Pour cela, on ne restreint pas la généralité en supposant que $\mathbf{E}$ est projectivement cofibrant et qu'il est projectivement stablement $\left(\mathbb{A}^{1}, \tau\right)$-fibrant. Dans ce cas, $\mathrm{L} a(\mathbf{E}) \simeq a(\mathbf{E})$ et le $T$-spectre symétrique $a(\mathbf{E})$ est un $\Omega$-spectre qui est $\left(\mathbb{A}^{1}, \tau\right)$-local niveau par niveau. (On utilise ici que $(a, b)$ est une équivalence de Quillen relativement à la structure projective non localisée, i.e., où les équivalences faibles sont les équivalences faibles de préfaisceaux niveau par niveau.) Ainsi, si $f: a(\mathbf{E}) \longrightarrow \mathbf{F}$ est une équivalence $\left(\mathbb{A}^{1}, \tau\right)$-locale stable avec $\mathbf{F}$ stablement $\left(\mathbb{A}^{1}, \tau\right)$-fibrant, le morphisme $f$ est en fait une équivalence faible de préfaisceaux niveau par niveau. Ceci montre que la composition de $\mathbf{E} \longrightarrow b(a(\mathbf{E})) \longrightarrow b(\mathbf{F})$ est une équivalence faible de préfaisceaux niveau par niveau. On a donc montré que l'unité de $(\mathrm{L} a, \mathrm{R} b)$ est inversible. Pour la counité, on raisonne de la même manière. Les détails sont laissés au lecteur.

Dans le reste de cet appendice, nous considérons un cas particulier que nous avions utilisé dans la Section 7. Notre but est de définir le foncteur « homologie rationnelle » $\mathbf{S H}^{\tau}(S) \longrightarrow \mathbf{D A}^{\tau}(S, \mathbb{Q})$ et de l'identifier avec un foncteur de localisation. Il s'agit là d'un résultat bien connu que nous reprenons pour la commodité du lecteur. (Des analogues avec transferts d'une partie des constructions ci-dessous figurent dans les travaux de O. Röndigs et P.-A. Østvær sur les modules au-dessus du spectre de la cohomologie motivique $[\mathbf{4 1}, \mathbf{4 2}]$; voir notamment la Remarque A.10 ci-dessous.)

Nous commençons par rappeler les constructions topologiques (ou plutôt simpliciales) que nous cherchons à étendre au contexte motivique. (Nous recommandons l'article [27] au lecteur qui 
souhaite voir plus de détails.) On note $\Delta^{\mathrm{op}} \mathcal{E} n s$ la catégorie des ensembles simpliciaux munie de sa structure de modèles canonique : les cofibrations sont les monomorphismes et les équivalences faibles sont les morphismes dont les réalisations géométriques sont des équivalences d'homotopie. On a aussi la variante pointée de cette catégorie de modèles qu'on notera $\Delta^{\mathrm{op}} \mathcal{E} n s_{\mathbf{e}}$. Étant donné un anneau $\Lambda$, on note $\Delta^{\mathrm{op}} \operatorname{Mod}(\Lambda)$ la catégorie des $\Lambda$-modules simpliciaux. On dispose d'une structure de modèles projective sur $\Delta^{\mathrm{op}} \operatorname{Mod}(\Lambda)$. Une flèche $K \longrightarrow L$ est une fibration si $K(\underline{\mathbf{n}}) \longrightarrow L(\underline{\mathbf{n}})$ est surjective pour $\underline{\mathbf{n}} \in \boldsymbol{\Delta}-\{\underline{\mathbf{0}}\}$. C'est une équivalence faible si C. $(K) \rightarrow$ C. $(L)$ est un quasiisomorphisme. (Bien entendu, C. $(-)$ désigne le foncteur « complexe de chaînes associé ».) Avec ces structures, on dispose d'un foncteur de Quillen à gauche (et de sa variante pointée) :

$$
-\otimes \Lambda: \Delta^{\mathrm{op}} \mathcal{E} n s \longrightarrow \Delta^{\mathrm{op}} \operatorname{Mod}(\Lambda) \quad\left(\text { et }-\otimes \Lambda: \Delta^{\mathrm{op}} \mathcal{E} n s_{\bullet} \longrightarrow \Delta^{\mathrm{op}} \operatorname{Mod}(\Lambda)\right) .
$$

Il est monoïdal, symétrique et unitaire lorsqu'on munit $\Delta^{\mathrm{op}} \mathcal{E} n s$ du produit cartésien (et $\Delta^{\mathrm{op}} \mathcal{E} n s_{\bullet}$ du smash produit). Son adjoint à droite est le foncteur d'oubli Oub qui associe à un module simplicial l'ensemble simplicial sous-jacent (pointé par 0).

Notons $\mathbf{C p l}_{\geq 0}(\Lambda)$ la catégorie des complexes de $\Lambda$-modules qui sont nuls en degrés homologiques strictement négatifs. C'est aussi naturellement une catégorie de modèles : les fibrations sont les surjections en degrés strictement positifs et les équivalences faibles sont les quasiisomorphismes. On dispose d'un couple d'équivalences de catégories inverses l'une de l'autre

$$
(\mathrm{N}, \Gamma): \Delta^{\mathrm{op}} \operatorname{Mod}(\Lambda) \rightleftarrows \mathbf{C p l}_{\geq 0}(\Lambda) \text {. }
$$

C'est la correspondance de Dold-Kan (cf. [16, Chap. III, Cor. 2.3]). Elle respecte les structures de modèles. Autrement dit, le foncteur $\mathrm{N}$ préserve et détecte les équivalences faibles, mais aussi les fibrations et les cofibrations. Par contre, on fera attention que les foncteurs $\mathrm{N}$ et $\Gamma$ ne sont pas monoïdaux. Ils le sont toutefois à homotopie près. En effet, on a des quasi-isomorphismes

$$
\mathrm{m}: \mathrm{N}_{\bullet}(E) \otimes \mathrm{N}_{\bullet}(F) \longrightarrow \mathrm{N}_{\bullet}(E \otimes F) \quad \text { et } \quad \mathrm{cm}: \mathrm{N}_{\bullet}(E \otimes F) \longrightarrow \mathrm{N}_{\bullet}(E) \otimes \mathrm{N}_{\bullet}(F),
$$

binaturels en $E, F \in \Delta^{\mathrm{op}} \operatorname{Mod}(\Lambda)$ et quasi-inverses l'un de l'autre. (En fait, on a aussi $\mathrm{cm} \circ \mathrm{m}=$ id.) De plus, les morphismes $\mathrm{m}$ (resp. $\mathrm{cm}$ ) ci-dessus font de $\mathrm{N}$ un foncteur pseudo-monoïdal, symétrique et unitaire (resp. pseudo-comonoïdal et unitaire). Pour plus de détails, on renvoie le lecteur à $[\mathbf{5 2}, \S 8.5]$.

Étant donné un entier naturel $n \in \mathbb{N}$, on note, comme de coutume, $\Delta^{n}$ l'ensemble simplicial représenté par $\underline{\mathbf{n}} \in \boldsymbol{\Delta}$. (On conviendra aussi que $\Delta^{-1}$ est l'ensemble simplicial vide.) L'ensemble simplicial $\partial \Delta^{n}$ est alors défini comme étant l'union des images des $n+1$ inclusions $\Delta^{n-1} \hookrightarrow \Delta^{n}$. Le quotient $\Delta^{n} / \partial \Delta^{n}$, vu comme ensemble simplicial pointé, est noté $S^{n}$. C'est la sphère simpliciale de dimension $n$.

On forme les catégories de $S^{1}$-spectres symétriques $\operatorname{Spt}_{\mathrm{S}^{1}}^{\Sigma}\left(\Delta^{\mathrm{op}} \mathcal{E} n s_{\bullet}\right)$ et $\mathbf{S p t}_{\mathrm{S}^{1}}^{\Sigma}\left(\boldsymbol{\Delta}^{\mathrm{op}} \operatorname{Mod}(\Lambda)\right) \operatorname{mu}-$ nies de leurs structures de modèles projectives stables (cf. $[\mathbf{2 0 , 2 1 ]}$ ). On a alors un foncteur de Quillen à gauche

$$
-\otimes \Lambda: \operatorname{Spt}_{\mathrm{S}^{1}}^{\Sigma}\left(\Delta^{\mathrm{op}} \varepsilon n s_{\bullet}\right) \longrightarrow \mathbf{S p t}_{\mathrm{S}^{1}}^{\Sigma}\left(\Delta^{\mathrm{op}} \operatorname{Mod}(\Lambda)\right)
$$

Il est monoïdal, symétrique et unitaire.

On forme aussi la catégorie $\mathbf{S p t}_{\Lambda[1]}^{\Sigma}\left(\mathbf{C p l}_{\geq 0}(\Lambda)\right)$ des $\Lambda$ [1]-spectres symétriques. Elle admet une structure de modèles stable. On dispose d'un foncteur monoïdal, symétrique et unitaire

$$
\mathrm{T}: \mathbf{S p t}_{\Lambda[1]}^{\Sigma}\left(\mathbf{C p l}_{\geq 0}(\Lambda)\right) \longrightarrow \mathbf{C p l}(\Lambda)
$$

qui envoie un $\Lambda[1]$-spectre $\mathbf{E}$ sur la colimite de la $\mathbb{N}$-suite

$$
\left\{\mathbf{E}_{0} \stackrel{\gamma_{0}^{\prime}}{\longrightarrow} \mathbf{E}_{1}[-1] \stackrel{\gamma_{1}^{\prime}}{\longrightarrow} \cdots \stackrel{\gamma_{n-1}^{\prime}}{\longrightarrow} \mathbf{E}_{n}[-n] \stackrel{\gamma_{n}^{\prime}}{\longrightarrow} \cdots\right\},
$$

où $\gamma_{n}^{\prime}: \mathbf{E}_{n} \longrightarrow \mathbf{E}_{n+1}[-1]$ est le morphisme déduit du $n$-ième morphisme d'assemblage du spectre E. Ce foncteur est une équivalence de Quillen à gauche lorsqu'on munit $\mathbf{C p l}(\Lambda)$ des sa structure projective. Son adjoint à droite associe à un complexe de $\Lambda$-modules $K$ le $\Lambda[1]$-spectre symétrique $\left(\tau_{\geq-n} K[n]\right)_{n \in \mathbb{N}}$ où $\Sigma_{n}$ agit au niveau $n$ par multiplication par la signature. 
Par ailleurs, le foncteur pseudo-monoïdal symétrique $\mathrm{N}$ induit un foncteur pseudo-monoïdal symétrique

$$
\mathrm{N}: \operatorname{Spt}_{\mathrm{S}^{1}}^{\Sigma}\left(\Delta^{\mathrm{op}} \operatorname{Mod}(\Lambda)\right) \longrightarrow \operatorname{Spt}_{\Lambda[1]}^{\Sigma}\left(\mathbf{C p p}_{\geq 0}(\Lambda)\right)
$$

(Remarquer en effet que $\mathrm{N}\left(\mathrm{S}^{1} \otimes \Lambda\right)=\Lambda[1]$.) Ce foncteur $\mathrm{N}$ n'est pas une équivalence de catégories. Cependant, il l'est au niveau des catégories homotopiques. Autrement dit, il préserve les équivalences faibles stables et le foncteur induit

$$
\mathrm{N}: \mathbf{H o}_{s t}\left(\mathbf{S p t}_{\mathrm{S}^{1}}^{\Sigma}\left(\Delta^{\mathrm{op}} \operatorname{Mod}(\Lambda)\right)\right) \stackrel{\sim}{\longrightarrow} \mathbf{H o}_{s t}\left(\mathbf{S p t}_{\Lambda[1]}^{\Sigma}\left(\mathbf{C p l}_{\geq 0}(\Lambda)\right)\right) \text {. }
$$

est une équivalence de catégories monoïdales, symétriques et unitaires.

En mettant ceci ensemble, on obtient la chaîne d'équivalences de catégories monoïdales symétriques et unitaires

$$
\mathbf{H o}_{s t}\left(\mathbf{S p t}_{\mathrm{S}^{1}}^{\Sigma}\left(\boldsymbol{\Delta}^{\mathrm{op}} \operatorname{Mod}(\Lambda)\right)\right) \stackrel{\mathrm{N}}{\sim} \mathbf{H o}_{s t}\left(\mathbf{S p t}_{\Lambda[1]}^{\Sigma}\left(\mathbf{C p l}_{\geq 0}(\Lambda)\right)\right) \stackrel{\mathrm{T}}{\sim} \mathbf{D}(\Lambda)
$$

En composant avec le foncteur dérivé à gauche de (151), on obtient en fin de compte un foncteur monoïdal, symétrique et unitaire

$$
\mathrm{T} \circ \mathrm{N}(-\otimes \Lambda): \mathrm{SH}=\mathbf{H o}_{s t}\left(\mathbf{S p t}_{\mathrm{S}^{1}}^{\Sigma}\left(\Delta^{\mathrm{op}} \mathcal{E} n s_{\bullet}\right)\right) \longrightarrow \mathbf{D}(\Lambda) .
$$

On termine la discussion «topologique » avec un fait spécial au cas $\Lambda=\mathbb{Q}$. Pour l'énoncer, nous devons d'abord définir la structure de modèles rationnelle sur la catégorie $\operatorname{Spt}_{\mathrm{S}^{1}}^{\Sigma}\left(\Delta^{\mathrm{op}} \mathcal{E} n s_{\bullet}\right)$. Soit $\mathcal{E}$ un ensemble de représentants des classes d'isomorphismes des objets compacts de la catégorie triangulée $\mathrm{SH}=\mathbf{H o}_{s t}\left(\mathbf{S p t}_{\mathrm{S}^{1}}^{\Sigma}\left(\Delta^{\mathrm{op}} \mathcal{E} n s_{\bullet}\right)\right)$. On supposera que tout objet de $\mathcal{E}$ est projectivement cofibrant et stablement fibrant. Pour chaque $n \in \mathbb{N}-\{0\}$ et $\mathbf{E} \in \mathcal{E}$, on choisit un morphisme de $\mathrm{S}^{1}$-spectres $e_{n}: \mathbf{E} \longrightarrow \mathbf{E}$ qui relève l'endomorphisme $n \cdot \mathrm{id}_{\mathbf{E}}$ dans $\mathrm{SH}$. La structure projective rationnelle sur $\operatorname{Spt}_{\mathrm{S}^{1}}^{\Sigma}\left(\Delta^{\mathrm{op}} \mathcal{E} n s_{\bullet}\right)$ est alors la localisation de Bousfield suivant l'ensemble des flèches $\mathscr{R}=\left\{e_{n}: \mathbf{E} \longrightarrow \mathbf{E}, n \in \mathbb{N}-\{0\}\right.$ et $\left.\mathbf{E} \in \mathcal{E}\right\}$. Cette structure ne dépend pas des choix effectués. On note $\mathrm{SH}_{\mathbb{Q}}$ sa catégorie homotopique. Un objet $\mathbf{F} \in \mathrm{SH}$ est $\mathscr{R}$-local, et on dira alors $\mathbb{Q}$-local, si et seulement si $n \cdot \operatorname{id}_{\mathbf{F}}$ est un isomorphisme pour tout $n \in \mathbb{N}-\{0\}$. La $\mathbb{Q}$-localisation $\operatorname{Loc}_{\mathbb{Q}}(A)$ d'un objet $A \in \mathrm{SH}$ est donnée par la colimite homotopique d'une $\mathbb{N}$-suite

$$
\left\{A \stackrel{n_{0} \cdot \mathrm{id}_{A}}{\longrightarrow} A \stackrel{n_{1} \cdot \mathrm{id}_{A}}{\longrightarrow} \cdots \stackrel{n_{s-1} \cdot \mathrm{did}_{A}}{\longrightarrow} A \stackrel{n_{s} \cdot \mathrm{id}_{A}}{\longrightarrow} \cdots\right\}
$$

où $\left(n_{s}\right)_{s \in \mathbb{N}}$ est une suite d'entiers naturels non nuls telle que $\left(n_{0} \cdots n_{s}\right)_{s \in \mathbb{N}}$ est finale dans l'ensemble $\mathbb{N}^{\times}=\mathbb{N}-\{0\}$ ordonné par la relation de divisibilité. Le résultat suivant est bien connu.

Proposition A.8 - Le foncteur

$$
-\otimes \mathbb{Q}: \mathbf{S p t}_{\mathrm{S}^{1}}^{\Sigma}\left(\Delta^{\mathrm{op}} \mathcal{E} n s_{\bullet}\right) \longrightarrow \mathbf{S p t}_{\mathrm{S}^{1}}^{\Sigma}\left(\Delta^{\mathrm{op}} \operatorname{Mod}(\mathbb{Q})\right)
$$

est une équivalence de Quillen à gauche lorsqu'on munit $\mathbf{S p t}_{\mathrm{S}^{1}}^{\Sigma}\left(\Delta^{\mathrm{op}} \mathcal{E} n s_{\bullet}\right)$ de sa structure rationnelle.

Demonstration Toute flèche de $\mathscr{R}$ est envoyée sur un isomorphisme par le foncteur $\mathrm{T} \circ \mathrm{N}(-\otimes \mathbb{Q})$ : $\mathrm{SH} \longrightarrow \mathbf{D}(\mathbb{Q})$. Ceci montre que le foncteur (156) est un foncteur de Quillen à gauche lorsqu'on munit la source de sa structure rationnelle. Il reste donc à voir que le foncteur $\mathrm{T} \circ \mathrm{N}(-\otimes \mathbb{Q})$ : $\mathrm{SH}_{\mathbb{Q}} \longrightarrow \mathbf{D}(\mathbb{Q})$ est une équivalence de catégories. Or, il est bien connu que la catégorie triangulée $\mathrm{SH}$ est compactement engendrée par le spectre unité $\mathbb{1}=\operatorname{Sus}_{\mathrm{S}^{1}, \Sigma}^{0}\left(\mathrm{~S}^{0}\right)$. Vue la description $\mathrm{du}$ foncteur de localisation rationnelle, $\mathrm{SH}_{\mathbb{Q}}$ est encore compactement engendrée par $\mathbb{1}$ et on a $\operatorname{hom}_{\mathrm{SH}_{\mathbb{Q}}}(\mathbb{1}, \mathbb{1}[n])=\operatorname{hom}_{\mathrm{SH}}(\mathbb{1}, \mathbb{1}[n]) \otimes \mathbb{Q}$. Or, les groupes d'homotopie stables des sphères sont de torsion excepté en degré 0 . Il vient que

$$
\operatorname{hom}_{\mathrm{SH}_{\mathbf{Q}}}(\mathbb{1}, \mathbb{1}[n])=\left\{\begin{array}{ccc}
\mathbb{Q} & \text { si } & n=0, \\
0 & \text { si } & n \neq 0 .
\end{array}\right.
$$

Le résultat recherché s'ensuit puisque hom $\left.\operatorname{Do}_{\mathbf{Q}}\right)(\mathbb{Q}, \mathbb{Q}[n])$ est donné par la même formule et que $\mathbf{D}(\mathbb{Q})$ est aussi compactement engendrée par $\mathbb{Q}[0]$. (Utiliser par exemple [6, Lem. 1.3.32].) 
On cherche maintenant à étendre la discussion «topologique » ci-dessus au cadre motivique. On notera dans la suite $\mathfrak{M}$ (resp. $\mathfrak{M}_{\mathbb{Q}}$ ) la catégorie de modèles $\operatorname{Spt}_{\mathrm{S}^{1}}^{\Sigma}\left(\Delta^{\mathrm{op}} \mathcal{E} n s_{\bullet}\right)$ munie de sa structure projective stable (resp. de sa structure projective rationnelle). On notera aussi $\mathfrak{N}_{1}(\Lambda)$ et $\mathfrak{N}_{2}(\Lambda)$ les catégories de modèles $\mathbf{S p t}_{\mathrm{S}^{1}}^{\Sigma}\left(\Delta^{\mathrm{op}} \operatorname{Mod}(\Lambda)\right)$ et $\mathbf{S p t}_{\Lambda[1]}^{\Sigma}\left(\mathbf{C p l}_{\geq 0}(\Lambda)\right)$ munies de leurs structures projectives stables. La catégorie $\mathbf{S} \mathbf{H}_{\mathfrak{M}}^{\tau}(S)\left(\right.$ resp. $\left.\mathbf{S H}_{\mathfrak{M}_{\mathbb{Q}}}^{\tau}(S)\right)$ des $S$-motifs à valeurs dans $\mathfrak{M}\left(\right.$ resp. $\mathfrak{M}_{\mathbb{Q}}$ ) sera simplement notée $\mathbf{S} \mathbf{H}^{\tau}(S)$ (resp. $\left.\mathbf{S} \mathbf{H}_{\mathbb{Q}}^{\tau}(S)\right)$. On commence par le résultat suivant.

Lemme A.9 - Soit $S$ un schéma. Le foncteur

$$
\mathrm{N}: \operatorname{Spt}_{T}^{\Sigma}\left(\mathbf{P S h}\left(\operatorname{Sm} / S, \mathfrak{N}_{1}(\Lambda)\right)\right) \longrightarrow \operatorname{Spt}_{T}^{\Sigma}\left(\mathbf{P S h}\left(\operatorname{Sm} / S, \mathfrak{N}_{2}(\Lambda)\right)\right)
$$

préserve les équivalences $\left(\mathbb{A}^{1}, \tau\right)$-locales stables. De plus, le foncteur induit

$$
\mathrm{N}: \mathbf{S H}_{\mathfrak{N}_{1}(\Lambda)}^{\tau}(S) \longrightarrow \mathbf{S H}_{\mathfrak{N}_{2}(\Lambda)}^{\tau}(S)
$$

est une équivalence de catégories monö̈dales, symétriques et unitaires. (La variante effective de cet énoncé est aussi vraie.)

Demonstration La preuve de ce lemme est très technique et inélégante. Les difficultés à surmonter sont causées par le fait que l'équivalence de catégories (154) ne provient pas d'une équivalence de Quillen. Nous sommes donc obligés de faire des détours.

Puisque les catégories $\mathfrak{R}_{1}(\Lambda)$ et $\mathfrak{R}_{2}(\Lambda)$ sont additives, le préfaisceau $\left(\mathbb{P}_{S}^{1}, \infty_{S}\right) \otimes \mathbb{1}$ à valeurs dans ces catégories est projectivement cofibrant. (En effet, il est facteur direct de $\mathbb{P}_{S}^{1} \otimes \mathbb{1}$.) On peut donc prendre $T_{S}=\left(\mathbb{P}_{S}^{1}, \infty_{S}\right) \otimes \mathbb{1}$. Les $T$-spectres symétriques (ou pas) s'identifient alors aux $\left(\left(\mathbb{P}_{S}^{1}, \infty_{S}\right) \otimes-\right)$-spectres symétriques (ou pas).

On pose $\mathfrak{N}_{1}^{\prime}(\Lambda)=\mathbf{S p t}_{\mathrm{S}^{1}}\left(\Delta^{\mathrm{op}} \operatorname{Mod}(\Lambda)\right)$ et $\mathfrak{N}_{2}^{\prime}(\Lambda)=\mathbf{S p t}_{\Lambda[1]}\left(\mathbf{C p l}_{\geq 0}(\Lambda)\right)$. C'est les catégories de spectres non symétriques qu' on munit de leurs structures projectives stables. On a des équivalences de Quillen à gauche $-\otimes_{\{1\}} \Sigma: \mathfrak{N}_{i}^{\prime}(\Lambda) \longrightarrow \mathfrak{N}_{i}(\Lambda)$, pour $i \in\{1,2\}$. On en déduit, en raisonnant comme dans la preuve du Lemme A.7, des équivalences de Quillen à gauche

$$
-\otimes_{\{1\}} \Sigma: \operatorname{Spt}_{\left(\mathbb{P}^{1}, \infty\right)}^{\Sigma}\left(\mathbf{P S h}\left(\operatorname{Sm} / S, \Re_{i}^{\prime}(\Lambda)\right)\right) \longrightarrow \operatorname{Spt}_{\left(\mathbb{P}^{1}, \infty\right)}^{\Sigma}\left(\operatorname{PSh}\left(\operatorname{Sm} / S, \Re_{i}(\Lambda)\right)\right) .
$$

(Ci-dessus, le symbole $\mathbf{S p t}_{\left(\mathbb{P}^{1}, \infty\right)}^{\Sigma}$ désigne la catégorie des $\left(\left(\mathbb{P}_{S}^{1}, \infty_{S}\right) \otimes-\right)$-spectres symétriques ; cette distinction est nécessaire puisque les catégories $\mathfrak{N}_{1}^{\prime}(\Lambda)$ et $\mathfrak{N}_{2}^{\prime}(\Lambda)$ ne sont pas monoïdales symétriques.) Sachant que (157) préserve les équivalences faibles de préfaisceaux niveau par niveau, on se ramène aussitôt à montrer que le foncteur

$$
\mathrm{N}: \mathbf{S p t}_{\left(\mathbb{P}^{1}, \infty\right)}^{\Sigma}\left(\mathbf{P S h}\left(\operatorname{Sm} / S, \mathfrak{N}_{1}^{\prime}(\Lambda)\right)\right) \longrightarrow \operatorname{Spt}_{\left(\mathbb{P}^{1}, \infty\right)}^{\Sigma}\left(\operatorname{PSh}\left(\operatorname{Sm} / S, \mathfrak{N}_{2}^{\prime}(\Lambda)\right)\right)
$$

préserve les équivalences $\left(\mathbb{A}^{1}, \tau\right)$-locales et qu'il induit une équivalence entre les catégories homotopiques.

Via la correspondance de Dold-Kan $(\mathrm{N}, \Gamma)$, la transformation binaturelle $\mathrm{cm}$ de (150) détermine une transformation $\mathrm{m}: \Gamma(E) \otimes \Gamma(F) \longrightarrow \Gamma(E \otimes F)$ binaturelle en $E, F \in \mathbf{C p l}_{\geq 0}(\Lambda)$. Le foncteur $\Gamma$ devient ainsi un foncteur pseudo-monoïdal et unitaire. (On fera attention, qu'il n'est pas symétrique !) On peut alors l'étendre à un foncteur sur les spectres (non symétriques)

$$
\Gamma: \mathfrak{R}_{2}^{\prime}(\Lambda)=\mathbf{S p t}_{\Lambda[1]}\left(\mathbf{C p l}_{\geq 0}(\Lambda)\right) \longrightarrow \mathfrak{R}_{1}^{\prime}(\Lambda)=\mathbf{S p t}_{\mathrm{S}^{1}}\left(\Delta^{\mathrm{op}} \operatorname{Mod}(\Lambda)\right) .
$$

De plus, vu que $\mathrm{cm} \circ \mathrm{m}=\mathrm{id}$, on a un isomorphisme canonique $\mathrm{N} \circ \Gamma \simeq \mathrm{id}_{\mathfrak{R}_{2}^{\prime}(\Lambda)}$ entre endofoncteurs de $\mathfrak{R}_{2}^{\prime}(\Lambda)$. (Ceci n'est pas le cas pour l'endofoncteur composé $\Gamma \circ \mathrm{N}$.) Le foncteur $\Gamma$ induit un foncteur

$$
\Gamma: \operatorname{Spt}_{\left(\mathbb{P}^{1}, \infty\right)}^{\Sigma}\left(\mathbf{P S h}\left(\operatorname{Sm} / S, \mathfrak{R}_{2}^{\prime}(\Lambda)\right)\right) \longrightarrow \operatorname{Spt}_{\left(\mathbb{P}^{1}, \infty\right)}^{\Sigma}\left(\mathbf{P S h}\left(\operatorname{Sm} / S, \Re_{1}^{\prime}(\Lambda)\right)\right)
$$

et on a encore la relation $N \circ \Gamma \simeq$ id entre les foncteurs (159) et (161). Par ailleurs, ces deux foncteurs préservent les équivalences faibles de préfaisceaux niveau par niveau. On en déduit des foncteurs triangulés

$$
\mathbf{H o}_{n i v}\left(\operatorname{Spt}_{\left(\mathbb{P}^{1}, \infty\right)}^{\Sigma}\left(\operatorname{PSh}\left(\operatorname{Sm} / S, \mathfrak{\Re}_{1}(\Lambda)\right)\right)\right) \underset{\Gamma}{\stackrel{\mathrm{N}}{\rightleftarrows}} \mathbf{H o}_{n i v}\left(\operatorname{Spt}_{\left(\mathbb{P}^{1}, \infty\right)}^{\Sigma}\left(\operatorname{PSh}\left(\operatorname{Sm} / S, \mathfrak{N}_{2}(\Lambda)\right)\right)\right)
$$


qui vérifient la relation $\mathrm{N} \circ \Gamma \simeq \mathrm{id}$. (Ci-dessus, $\mathbf{H o}_{n i v}$ désigne les catégories homotopiques où l'on a inversé les équivalences faibles de préfaisceaux niveau par niveau.) Les catégories triangulées dans (162) sont compactement engendrées par les objets de la forme $\operatorname{Sus}_{\left(\mathbb{P}^{1}, \infty\right), \Sigma}^{n}(U \otimes \mathbb{1})$ avec $n \in \mathbb{N}$, $U \in \mathrm{Sm} / S$ et où $\mathbb{1} \in\left\{\operatorname{Sus}_{\mathrm{S}^{1}}^{0}(\Lambda), \operatorname{Sus}_{\Lambda[1]}^{0}(\Lambda)\right\}$. Par ailleurs, si $\mathbf{E}$ est un $\left(\left(\mathbb{P}_{S}^{1}, \infty_{S}\right) \otimes-\right)$-spectre symétrique, on a

$$
\pi_{0}\left(\operatorname{Sus}_{\left(\mathbb{P}^{1}, \infty\right), \Sigma}^{n}(U \otimes \mathbb{1}), \mathbf{E}\right)=\pi_{0}\left(\mathbf{E}_{n}(U)\right) .
$$

Vus les isomorphismes $\mathrm{N}(\mathbb{1}) \simeq \mathbb{1}$ et $\Gamma(\mathbb{1}) \simeq \mathbb{1}$ dans $\mathbf{H o}\left(\mathfrak{N}_{2}(\Lambda)\right)$ et $\mathbf{H o}\left(\Re_{1}(\Lambda)\right)$, on déduit des isomorphismes

$$
\mathrm{N}\left(\operatorname{Sus}_{\left(\mathbb{P}^{1}, \infty\right), \Sigma}^{n}(U \otimes \mathbb{1})\right) \simeq \operatorname{Sus}_{\left(\mathbb{P}^{1}, \infty\right), \Sigma}^{n}(U \otimes \mathbb{1}) \text { et } \Gamma\left(\operatorname{Sus}_{\left(\mathbb{P}^{1}, \infty\right), \Sigma}^{n}(U \otimes \mathbb{1})\right) \simeq \operatorname{Sus}_{\left(\mathbb{P}^{1}, \infty\right), \Sigma}^{n}(U \otimes \mathbb{1})
$$

dans les catégories homotopiques qui figurent dans (162). Il en découle aussitôt que les foncteurs $\mathrm{N}$ et $\Gamma$ dans (162) sont pleinement fidèles. La relation $\mathrm{N} \circ \Gamma \simeq$ id entraîne alors qu'ils sont des équivalences de catégories.

Il est maintenant aisé de conclure. En effet, les équivalences de catégories (162) préservent la classe des $\Omega$-spectres symétriques qui sont $\left(\mathbb{A}^{1}, \tau\right)$-locaux niveau par niveau. Ceci découle immédiatement des définitions. Il s'ensuit que les équivalences de catégories (162) préservent aussi les équivalences $\left(\mathbb{A}^{1}, \tau\right)$-locales puisque ces dernières sont caractérisées par la propriété d'induire des isomorphismes en leur appliquant le foncteur $\operatorname{hom}_{\mathbf{H o}_{\text {niv }}(\ldots)}(-, \mathbf{E})$ avec $\mathbf{E}$ un $\Omega$-spectre symétrique $\left(\mathbb{A}^{1}, \tau\right)$-local niveau par niveau.

Remarque A.10 - Dans [42, Th. 11(1)] le lecteur trouvera un analogue avec transferts du Lemme A.9 ci-dessus. Toutefois, faisant abstraction des transferts, il est utile de noter la différence technique suivante. Nous travaillons avec des $T$-spectres de préfaisceaux à valeurs dans les catégories de modèles stables de $S^{1}$-spectres ou de $\Lambda$ [1]-spectres. Dans [42], les auteurs suivent de plus près la recette de Jardine [28] consistant à prendre des $T$-spectres à valeurs dans des catégories de modèles instables. Bien entendu, les deux recettes aboutissent à des catégories de modèles Quillen-équivalentes : le point est que $T$ est $\mathbb{A}^{1}$-équivalent à une suspension. Néanmoins, cette différence explique les complications techniques rencontrées dans la preuve du Lemme A.9.

Par le Lemme A.7, l'équivalence de Quillen à gauche (152) induit une équivalence de Quillen sur les catégories de $T$-spectres symétriques en préfaisceaux sur $\mathrm{Sm} / S$. Étant donné que ce foncteur préserve les équivalences faibles de préfaisceaux niveau par niveau, il préserve aussi les équivalences $\left(\mathbb{A}^{1}, \tau\right)$-locales stables. Il induit donc un foncteur

$$
\mathrm{T}: \mathbf{S H}_{\mathfrak{N}_{2}(\Lambda)}^{\tau}(S) \longrightarrow \mathbf{D A}^{\tau}(S, \Lambda)
$$

qui est une équivalence de catégories monoïdales, symétriques et unitaires. Par le Lemme A.9, on a donc une chaînes d'équivalences de catégories monoïdales, symétriques et unitaires

$$
\mathbf{S H}_{\mathfrak{N}_{1}(\Lambda)}^{\tau}(S) \stackrel{\mathrm{N}}{\sim} \mathbf{S H}_{\mathfrak{N}_{2}(\Lambda)}^{\tau}(S) \stackrel{\mathrm{T}}{\sim} \mathbf{D A}^{\tau}(S, \Lambda) .
$$

Par ailleurs, le foncteur de Quillen à gauche (151) induit un foncteur monoïdal symétrique et unitaire $\mathbf{S H}^{\tau}(S) \longrightarrow \mathbf{S H}_{\mathfrak{N}_{1}(\Lambda)}^{\tau}(S)$. De ce qui précède, on déduit un foncteur monoïdal, symétrique et unitaire

$$
\mathrm{T} \circ \mathrm{N}(-\otimes \Lambda): \mathbf{S H}^{\tau}(S) \longrightarrow \mathbf{D A}^{\tau}(S, \Lambda) .
$$

C'est le foncteur « homologie à coefficients dans $\Lambda$ ». Le résultat suivant est une conséquence immédiate des constructions.

Lemme A.11 - Soit $f: T \longrightarrow S$ un morphisme de schémas.

(a) Le carré

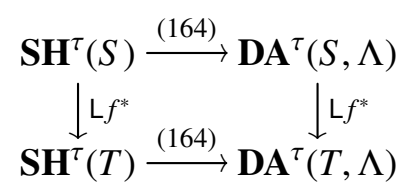


est commutatif à un isomorphisme canonique près.

(b) Supposons que f est lisse. Alors, le carré

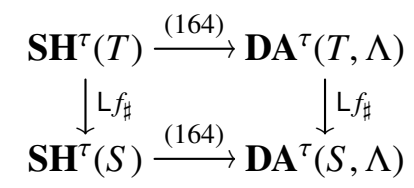

est commutatif à un isomorphisme canonique près.

À partir de maintenant, on se concentre sur le cas $\Lambda=\mathbb{Q}$. On rappelle que $\mathbf{S H}_{\mathbb{Q}}^{\tau}(S)$ désigne la catégorie $\mathbf{S H}_{\mathfrak{M}_{\mathbb{Q}}}^{\tau}(S)$ des $S$-motifs à coefficients dans la catégorie de modèles $\mathfrak{M}_{\mathbb{Q}}=\mathbf{S p t}_{S^{1}}^{\Sigma}\left(\Delta^{\mathrm{op}} \mathcal{E} n s_{\bullet}\right)$ munie de sa structure projective rationnelle. Étant donné que $-\otimes \mathbb{Q}: \mathfrak{M}_{\mathbb{Q}} \longrightarrow \mathfrak{N}_{1}(\mathbb{Q})$ est une équivalence de Quillen à gauche (voir la Proposition A.8), on déduit une équivalence de catégories monoïdales symétriques et unitaires $-\otimes \mathbb{Q}: \mathbf{S H}_{\mathbb{Q}}^{\tau}(S) \longrightarrow \mathbf{S H}_{\mathfrak{N}_{1}(\mathbb{Q})}(S)$. On obtient ainsi une équivalence de catégories monoïdales symétriques et unitaires

$$
\mathrm{T} \circ \mathrm{N}(-\otimes \mathbb{Q}): \mathbf{S H}_{\mathbb{Q}}^{\tau}(S) \stackrel{\sim}{\longrightarrow} \mathbf{D A}^{\tau}(S, \mathbb{Q}) .
$$

On aura besoin du résultat suivant.

Lemme A.12 - La catégorie $\operatorname{Spt}_{T}^{\Sigma}\left(\mathbf{P S h}\left(\operatorname{Sm} / S, \mathfrak{M}_{\mathbb{Q}}\right)\right)$, munie de sa structure projective $\left(\mathbb{A}^{1}, \tau\right)$ locale, est la localisation de Bousfield de la catégorie $\operatorname{Spt}_{T}^{\Sigma}(\mathbf{P S h}(\operatorname{Sm} / S, \mathfrak{M}))$, munie de sa structure projective $\left(\mathbb{A}^{1}, \tau\right)$-locale, suivant l'ensemble des flèches

$$
\operatorname{Sus}_{T, \Sigma}^{m}\left(U \otimes e_{n}\right): \operatorname{Sus}_{T, \Sigma}^{m}(U \otimes E) \longrightarrow \operatorname{Sus}_{T, \Sigma}^{m}(U \otimes E)
$$

pour $U \in \mathrm{Sm} / S, E \in \mathcal{E}, m \in \mathbb{N}$ et $n \in \mathbb{N}-\{0\}$.

Demonstration Ceci est une conséquence immédiate de la commutativité des localisations de Bousfield.

Proposition A.13 - Supposons que l'une des deux conditions suivantes est satisfaite :

(i) $\tau=$ Nis et la dimension de Krull de $S$ est finie;

(ii) $\tau$ = ét, la dimension de Krull de $S$ est finie et la p-dimension cohomologique ponctuelle de $S$ est uniformément bornée pour p parcourant l'ensemble des nombres premiers.

Alors, la $\mathbb{Q}$-localisation, $\operatorname{Loc}_{\mathbb{Q}}(\mathbf{E})$, d'un objet $\mathbf{E} \in \mathbf{S H}^{\tau}(S)$ est donnée par la colimite homotopique d'une $\mathbb{N}$-suite

$$
\left\{\mathbf{E} \stackrel{n_{0} \cdot \mathrm{id}_{\mathbf{E}}}{\longrightarrow} \mathbf{E} \stackrel{n_{1} \cdot \mathrm{id}_{\mathbf{E}}}{\longrightarrow} \cdots \stackrel{n_{s-1} \cdot \mathrm{id}_{\mathbf{E}}}{\longrightarrow} \mathbf{E} \stackrel{n_{s} \cdot \mathrm{id}_{\mathbf{E}}}{\longrightarrow} \cdots\right\}
$$

où $\left(n_{s}\right)_{s \in \mathbb{N}}$ est une suite d'entiers naturels non nuls telle que $\left(n_{0} \cdots n_{s}\right)_{s \in \mathbb{N}}$ est finale dans l'ensemble $\mathbb{N}^{\times}=\mathbb{N}-\{0\}$ ordonné par la relation de divisibilité.

Demonstration Notons $\mathscr{R}(S)$ la classe des flèches $\operatorname{Sus}_{T, \Sigma}^{m}\left(U \otimes e_{n}\right)$ comme dans le Lemme A.12. Appelons $\mathbf{F}$ la colimite homotopique de la $\mathbb{N}$-suite de l'énoncé. Nous montrons d'abord que le $T$ spectre symétrique $\mathbf{F}$ est $\mathbb{Q}$-local. Par le Lemme $\mathrm{A} .12$ ceci revient à dire qu'il est $\mathscr{R}(S)$-local, i.e.,

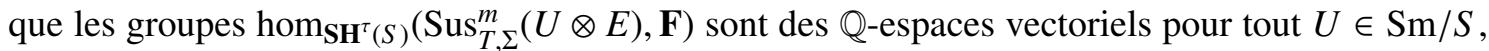
$m \in \mathbb{N}$ et $E \in \mathcal{E}$. Or, sous les hypothèses de l'énoncé, les objets $\operatorname{Sus}_{T, \Sigma}^{m}(U \otimes E)$ sont compacts dans $\mathbf{S H}^{\tau}(S)$ (cf. [5, Th. 4.5.67]). Il vient que nos groupes sont les colimites des $\mathbb{N}$-suites

$$
\left\{\operatorname{hom}_{\mathbf{S H}} \mathbf{T}^{\tau}(S)\left(\operatorname{Sus}_{T, \Sigma}^{m}(U \otimes E), \mathbf{E}\right) ; n_{s} \cdot \mathrm{id}\right\}_{s \in \mathbb{N}} .
$$

La condition sur la suite des entiers $\left(n_{s}\right)_{s \in \mathbb{N}}$ entraîne que ces groupes sont uniquement divisibles.

Il reste à voir que $\mathbf{E} \longrightarrow \mathbf{F}$ est une équivalence $\mathscr{R}(S)$-locale. Or, la classe des équivalences $\mathscr{R}(S)$-locales est stable par la composition des $\mathbb{N}$-suites. Il suffit donc de prouver que $m \cdot \operatorname{id}_{\mathbf{E}}$ est une équivalence $\mathscr{R}(S)$-locale pour tout $m \in \mathbb{N}-\{0\}$. Pour cela, il suffit de prouver que l'image de $m \cdot \mathrm{id}_{\mathbf{E}}$ par le foncteur $\mathrm{T} \circ \mathrm{N}(-\otimes \mathbb{Q})$ est un isomorphisme. Or, dans $\mathbf{D A}^{\tau}(S, \mathbb{Q})$, le morphisme $m \cdot \mathrm{id}: \mathrm{T} \circ \mathrm{N}(\mathbf{E} \otimes \mathbb{Q}) \longrightarrow \mathrm{T} \circ \mathrm{N}(\mathbf{E} \otimes \mathbb{Q})$ est clairement inversible. 
Corollaire A.14 - Gardons les hypothèses de la Proposition A.13.

(a) Soit $f: T \longrightarrow S$ un morphisme de type fini. Alors la face carrée

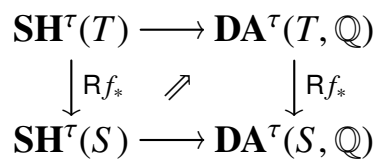

est inversible.

(b) Soit $i: Z \hookrightarrow S$ une immersion fermée. Alors, la face carrée

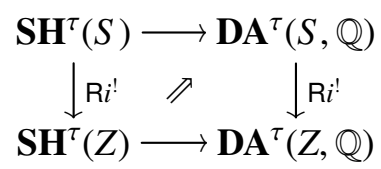

est inversible.

Demonstration Bien entendu, il suffit de prouver la commutation de $\mathrm{R} f_{*}$ et $\mathrm{R} i$ ! avec les foncteurs de localisation $\mathbf{S H}^{\tau}(-) \longrightarrow \mathbf{S H}_{\odot}^{\tau}(-)$. Soit $\mathbf{E} \in \mathbf{S H}^{\tau}(T)$. Il faut alors montrer que le morphisme canonique $\mathrm{R} f_{*} \mathbf{E} \longrightarrow \mathrm{R} f_{*} \operatorname{Loc}(\mathbf{E})$ est un isomorphisme dans $\mathbf{S H}_{\mathbb{Q}}^{\tau}(S)$. Sous les hypothèses de la Proposition A.13, le foncteur $\mathrm{R} f_{*}: \mathbf{S H}^{\tau}(T) \longrightarrow \mathbf{S H}^{\tau}(S)$ commutent aux sommes infinies. (Utiliser [4, Lem. 2.1.159] joint à [5, Th. 4.5.67].) En utilisant la Proposition A.13, on déduit alors que $\operatorname{Loc}_{\mathbb{Q}}\left(\mathrm{R} f_{*} \mathbf{E}\right) \simeq \mathrm{R} f_{*} \operatorname{Loc}_{\mathbb{Q}}(\mathbf{E})$. Or, le morphisme $\mathrm{R} f_{*} \mathbf{E} \longrightarrow \operatorname{Loc}_{\mathbb{Q}}\left(\mathrm{R} f_{*} \mathbf{E}\right)$ est clairement inversible dans $\mathbf{S H}_{\mathbb{Q}}^{\tau}(S)$. Ceci démontre la première moitié du corollaire.

Pour la seconde moitié, on utilise le triangle distingué de localisation associé à l'immersion fermée $i$ et son immersion ouverte complémentaire $j$. On se ramène ainsi à montrer la commutation avec le foncteur $\mathrm{R} j_{*}$, ce qui est un cas particulier de la première moitié du corollaire.

Nous avons en fait le résultat plus complet suivant.

Theoreme A.15 - Gardons les hypothèses de la Proposition A.13. Alors, les foncteurs T o $\mathrm{N}(-\otimes \mathbb{Q}): \mathbf{S H}^{\tau}(-) \longrightarrow \mathbf{D A}^{\tau}(-, \mathbb{Q})$ commute aux opérations $f^{*}, f_{*}, f_{!}$et $f^{!}$pour tout $S$-morphisme quasi-projectif $f$.

Demonstration On donne une esquisse de démonstration. D'après le Lemme A.11 et le Corollaire A.14, ces commutations sont établies pour les opérations $f^{*}, f_{*}, g_{\sharp}$ et $i$ avec $f$ quasi-projectif, $g$ lisse et $i$ une immersion fermée. On en déduit la commutation avec les équivalences de Thom. En utilisant que $g^{!} \simeq \operatorname{Th}\left(\Omega_{g}\right) g^{*}$ pour $g$ lisse, on obtient alors la commutation avec l'opération $f^{!}$pour $f$ quasi-projectif. En utilisant que $f_{!} \simeq f_{*}$ pour $f$ projectif et que $j_{!}=j_{\sharp}$ pour $j$ une immersion ouverte, on obtient la commutation avec l'opération $f$ ! pour $f$ quasi-projectif.

\section{Annexe B}

\section{Motifs étales avec et sans transferts}

Dans ce deuxième annexe, nous présentons une preuve du résultat suivant qui est dû à CisinskiDéglise [11] dans le cas où $\Lambda$ est une $\mathbb{Q}$-algèbre. 
Theoreme B.1 - Soient $S$ schéma normal et universellement japonais ${ }^{(10)}$, et $\Lambda$ un anneau commutatif. On suppose que l'Hypothèse 7.3 est satisfaite. Alors, le foncteur

$$
\mathrm{La}_{t r}: \mathbf{D A}^{\mathrm{e} t}(S, \Lambda) \longrightarrow \mathbf{D M}^{\mathrm{ét}}(S, \Lambda)
$$

est une équivalence de catégories.

Lorsque $\Lambda=\mathbb{Q}$ et $S$ est le spectre d'un corps parfait, un résultat plus précis a été annoncé par Morel dans une note non publiée (cf. [35]). Toujours pour $\Lambda=\mathbb{Q}$, Cisinski et Déglise en donnent une preuve dans $[11, \S 15.2]$ lorsque $S$ est excellent et unibranche. Leur méthode utilise la $K$ théorie algébrique comme prévu par Morel. Dans [8, Ann. B] nous avons simplifié la méthode de Cisinski-Déglise dans le cas où $S$ est de caractéristiques résiduelles nulles, ce qui nous a permis d'obtenir également la variante effective. Là encore, nous avons supposé que $\Lambda$ était une $\mathbb{Q}$-algèbre.

L'ingrédient nouveau qui nous a permis d'obtenir une variante « entière » du théorème de Cisinski-Déglise est le théorème de rigidité relatif (cf. le Théorème 4.1). Ce théorème permet de traiter le cas où $\Lambda$ est de torsion (cf. la Proposition B.2 ci-dessous). Un dévissage simple nous ramène alors au cas où $\Lambda$ est une $\mathbb{Q}$-algèbre. Au lieu d'invoquer [11], nous reprenons la méthode simplifiée de [8, Ann. B] en éliminant l'hypothèse sur les caractéristiques résiduelles de $S$. Ceci est désormais possible grâce au Théorème 3.9 qui nous oblige cependant de travailler stablement.

Proposition B.2 - Soient $S$ un schéma normal et $\Lambda$ une $\mathbb{Z} / N \mathbb{Z}$-algèbre avec $N \in \mathbb{N}$ un entier inversible dans $\mathcal{O}_{S}$. On suppose qu'en tout point géométrique $\bar{x}$ de $S$ la p-dimension cohomologique ponctuelle de l'hensélisé strict de $S$ en $\bar{x}$ est finie si p est un nombre premier divisant $N$. Alors, le foncteur $\mathrm{La}_{t r}: \mathbf{D A}^{\mathrm{ét}}(S, \Lambda) \longrightarrow \mathbf{D M}^{\mathrm{ét}}(S, \Lambda)$ est une équivalence de catégories.

Demonstration D'après la Proposition 3.7, $\mathbf{D A}^{\text {ét }}(S, \Lambda)$ coïncide avec sa plus petite sous-catégorie triangulée stable par sommes infinies et contenant les objets de la forme $\operatorname{Sus}_{T, \Sigma}^{n}(X \otimes \Lambda)$ avec $n \in \mathbb{N}$ et $X \in \mathrm{Sm} / S$. De même, $\mathbf{D M}^{\text {ét }}(S, \Lambda)$ coïncide avec sa plus petite sous-catégorie triangulée stable par sommes infinies et contenant les objets de la forme $\operatorname{Sus}_{T^{t r}, \Sigma}^{n}\left(\Lambda_{t r}(X)\right)$ avec $n \in \mathbb{N}$ et $X \in \mathrm{Sm} / S$, et la preuve de ceci se calque sur celle de la Proposition 3.7. (Bien entendu, $T^{t r}=\mathrm{a}_{t r}(T)$ est le motif de Tate avec transferts et $\Lambda_{t r}(X)=\mathbb{Z}_{t r}(X) \otimes_{\mathbb{Z}} \Lambda$ est le préfaisceau avec transferts représenté par $X$.) Par ailleurs, le foncteur $\mathrm{La}_{t r}$ commute aux sommes infinies et on a $\operatorname{La}_{t r}\left(\operatorname{Sus}_{T, \Sigma}^{p}(X \otimes \Lambda)\right)=$ $\operatorname{Sus}_{T^{t r}, \Sigma}^{p}\left(\Lambda_{t r}(X)\right)$. Il est donc suffisant de montrer que le foncteur $\mathrm{La}_{t r}$ est pleinement fidèle.

D'après le Théorème 4.1, le foncteur évident $\mathrm{L}_{S}^{*}: \mathbf{D}^{\text {ét }}(S, \Lambda) \longrightarrow \mathbf{D} \mathbf{A}^{\text {ét }}(S, \Lambda)$ est une équivalence de catégories. En particulier, $\mathbf{D A}{ }^{\text {ét }}(S, \Lambda)$ coïncide avec sa plus petite sous-catégorie triangulée stable par sommes infinies et contenant les objets de la forme $\operatorname{Sus}_{T}^{0}(U \otimes \Lambda)$ avec $U$ un $S$-schéma étale. Pour terminer la preuve, il est donc suffisant de montrer l'homomorphisme

$$
\operatorname{hom}_{\mathbf{D A}} \mathbf{\mathrm { et }}^{(S, \Lambda)}\left(\operatorname{Sus}_{T, \Sigma}^{0}(U \otimes \Lambda), \mathbf{F}\right) \longrightarrow \operatorname{hom}_{\mathbf{D M}}^{\mathrm{et}(S, \Lambda)}\left(\operatorname{Sus}_{T^{t r}, \Sigma}^{0}\left(\Lambda_{t r}(U)\right), \operatorname{La}_{t r} \mathbf{F}\right),
$$

induit par le foncteur Latr , est bijectif pour tout $U \in \mathrm{Et} / S$ et $\mathbf{F} \in \operatorname{Spt}_{T}^{\Sigma}(\mathbf{C p l}(\mathbf{P S h}(\operatorname{Sm}, \Lambda)))$.

On ne restreint pas la généralité en supposant que $\mathbf{F}$ est projectivement cofibrant et stablement ( $\mathbb{A}^{1}$, ét)-fibrant. Nous allons montrer que le morphisme $\mathbf{F}_{n} \longrightarrow \mathrm{o}_{t r} \mathrm{a}_{t r} \mathbf{F}_{n}$ est une équivalence ét-locale de préfaisceaux pour tout $n \in \mathbb{N}$. Or, d'après le Corollaire 4.14, le morphisme naturel $\iota^{*} \iota_{*} \mathbf{F}_{n} \longrightarrow \mathbf{F}_{n}$ est une équivalence ét-locale pour tout $n \in \mathbb{N}$. (On rappelle que $\iota: \mathrm{Et} / S \hookrightarrow \mathrm{Sm} / S$ est l'inclusion évidente.) Il suffit donc de montrer que le morphisme naturel $\left(\iota^{*} \iota_{*} \mathbf{F}_{n}\right) \longrightarrow \mathrm{o}_{t r} \mathrm{La}_{t r}\left(\iota^{*} \iota_{*} \mathbf{F}_{n}\right)$ est une équivalence ét-locale de complexes de préfaisceaux. Il est plus général de montrer que

10. Rappelons qu'un schéma $S$ est dit universellement japonais si pour tout $S$-schéma de type fini et intègre $X$, le normalisé de $X$ dans son corps de fraction est encore un $S$-schéma de type fini. À vrai dire, cette propriété de $S$ n'est pas indispensable pour la validitié du Théorème B.1. En effet, il est facile de voir qu'il suffit de montrer que (169) induit une équivalence entre les sous-catégories triangulées de objets constructibles, et cela dans le cas où $S$ est supposé affine. Or, on peut écrire un tel $S$ comme une limite projective cofiltrante de schémas affines, normaux et essentiellement de type fini sur $\mathbb{Z}$, et donc en particulier universellement japonais. Le Corollaire 3.22 et son analogue avec transferts permettent ainsi de ramener le cas général au cas universellement japonais. Nous laisserons les détails au lecteur intéressé. 
$\iota^{*} G \longrightarrow \mathrm{o}_{t r} \mathrm{a}_{t r} \iota^{*} G$ est une équivalence ét-locale pour tout complexe de préfaisceaux projectivement cofibrant $G$ sur Et $/ S$. Or, le morphisme a aét $\left(\imath^{*} G\right) \longrightarrow \mathrm{a}_{\text {ét }}\left(\mathrm{o}_{t r} \mathrm{a}_{t r} r^{*} G\right)$ est un isomorphisme. Pour voir cela, on peut supposer que $G=V \otimes \Lambda$ avec $V \in \mathrm{Et} / S$. Il s'agit alors de montrer que $V \otimes \Lambda \longrightarrow \Lambda_{t r}(V)$ induit un isomorphisme sur les fibres. Mais si $X$ est un $S$-schéma lisse et $\bar{x}$ est un point géométrique de $X, \operatorname{Spec}\left(\mathcal{O}_{X, \bar{x}}^{h s}\right) \times_{S} V$ est une somme disjointe de copies de $\operatorname{Spec}\left(\mathcal{O}_{X, \bar{x}}^{h s}\right)$ et d'un morphisme étale non surjectif. Il s'ensuit immédiatement que $\operatorname{Cor}_{S}\left(\operatorname{Spec}\left(\mathcal{O}_{X, \bar{x}}^{h s}\right), V\right)=$ $\operatorname{hom}_{S}\left(\operatorname{Spec}\left(\mathcal{O}_{X, \bar{x}}^{h s}\right), V\right) \otimes \mathbb{Z}$. (On utilise ici que $\operatorname{Spec}\left(\mathcal{O}_{X, \bar{x}}^{h s}\right)$ est normal et intègre de sorte qu'une $S$-correspondance finie de $\operatorname{Spec}\left(\mathcal{O}_{X, \bar{x}}^{h s}\right)$ dans $V$ est donnée par une combinaison linéaire des copies de $\operatorname{Spec}\left(\mathcal{O}_{X, \bar{x}}^{h s}\right)$ qui se trouvent dans $\operatorname{Spec}\left(\mathcal{O}_{X, \bar{x}}^{h s}\right) \times_{S} V$.) Ceci démontre le résultat recherché.

Sachant que les morphismes $\mathbf{F}_{n} \longrightarrow \mathrm{o}_{t r} \mathrm{a}_{t r} \mathbf{F}_{n}$ sont des équivalences ét-locales de préfaisceaux, il est aisé de conclure. En effet, le $T^{t r}$-spectre $\mathrm{a}_{t r} \mathbf{F}$ est stablement ( $\mathbb{A}^{1}$, ét)-local puisque ceci se vérifie après application de $\mathrm{o}_{t r}$ et que $\mathbf{F}$ est stablement $\left(\mathbb{A}^{1}\right.$, ét)-fibrant. Le morphisme (167) s'écrit alors

$$
\operatorname{hom}_{\mathbf{D A}^{\text {eff,ét }(S, \Lambda)}}\left(U \otimes \Lambda, \mathbf{F}_{0}\right) \longrightarrow \operatorname{hom}_{\mathbf{D M}^{\text {eff,ét }}(S, \Lambda)}\left(\Lambda_{t r}(U), \mathrm{a}_{t r} \mathbf{F}_{0}\right) .
$$

Par adjonction, le second membre peut aussi s'écrire $\operatorname{hom}_{\mathbf{D A}^{\text {eff, ett }}(S, \Lambda)}\left(U \otimes \Lambda, \mathrm{o}_{t r} \mathrm{a}_{t r} \mathbf{F}_{0}\right)$. Le lemme en découle puisque on a montré que $\mathbf{F}_{0} \longrightarrow \mathrm{o}_{t r} \mathrm{a}_{t r} \mathbf{F}_{0}$ est une équivalence ét-locale.

On utilisera la Proposition B.2 pour établir la réduction suivante.

Corollaire B.3 - Il suffit de démontrer le Théorème B.1 lorsque $\Lambda$ est une $\mathbb{Q}$-algèbre.

Demonstration D'après la Proposition 3.19, $\mathbf{D A}^{\mathrm{e}}(S, \Lambda)$ est compactement engendrée par les objets de la forme $\operatorname{Sus}_{T, \Sigma}^{n}(X \otimes \Lambda)$ où $n \in \mathbb{N}$ et $X \in \operatorname{Sm} / S$. De même, DM $\mathbf{M}^{\text {ét }}(S, \Lambda)$ est compactement engendrée par les objets de la forme $\operatorname{Sus}_{T^{t r}, \Sigma}^{n}\left(\Lambda_{t r}(X)\right)$ où $n \in \mathbb{N}$ et $X \in \mathrm{Sm} / S$, et ceci se démontre en calquant la preuve de la Proposition 3.19. Vu que $\mathrm{La}_{t r}$ commute aux sommes infinies et que $\operatorname{La}_{t r}\left(\operatorname{Sus}_{T, \Sigma}^{n}(X \otimes \Lambda)\right)=\operatorname{Sus}_{T^{t r}, \Sigma}^{n}\left(\Lambda_{t r}(X)\right)$, il est suffisant de montrer que Lat ${ }_{t r}$ est pleinement fidèle et plus précisément que l'homomorphisme

$$
\operatorname{hom}_{\mathbf{D A}^{\mathrm{et}}(S, \Lambda)}(A, M) \longrightarrow \operatorname{hom}_{\mathbf{D M}^{\mathrm{et}}(S, \Lambda)}\left(\mathrm{La}_{t r}(A), \mathrm{La}_{t r}(M)\right)
$$

est bijectif pour tout $A, M \in \mathbf{D A}^{\text {ét }}(S, \Lambda)$ avec $A$ compact. Il suffit même de prendre $A=\operatorname{Sus}_{T, \Sigma}^{n}(X \otimes$ $\Lambda$ ) de sorte qu'on peut supposer que $A=A_{0} \otimes_{\Lambda_{0}} \Lambda$ avec $\Lambda_{0} \subset \mathbb{Q}$ un sous-anneau satisfaisant à l'Hypothèse 7.3 et $A_{0} \in \mathbf{D A}^{\text {ét }}\left(S, \Lambda_{0}\right)$ un objet compact. Or, on a un isomorphisme d'adjonction $\operatorname{hom}_{\mathbf{D A}^{\mathrm{et}}(S, \Lambda)}\left(A_{0} \otimes_{\Lambda_{0}} \Lambda, M\right) \simeq \operatorname{hom}_{\mathbf{D A}^{\mathrm{et}}\left(S, \Lambda_{0}\right)}\left(A_{0}, M\right)$ et de même pour $\mathbf{D M}^{\mathrm{ét}}(S,-)$. On peut donc remplacer $\Lambda$ par $\Lambda_{0}$. Quitte à faire cela, on peut supposer que $\Lambda$ est plat sur $\mathbb{Z}$.

Vu le triangle distingué $\mathbb{Z} \longrightarrow \mathbb{Q} \longrightarrow \mathbb{Q} / \mathbb{Z} \longrightarrow \mathbb{Z}[1]$, on voit qu'il suffit de prouver que (168) est inversible pour $M \otimes_{\mathbb{Z}} \mathbb{Q}$ et $M \otimes_{\mathbb{Z}} \mathbb{Q} / \mathbb{Z}$. Par ailleurs, $\mathbb{Q} / \mathbb{Z}=\oplus_{p} \mathbb{Z}[1 / p] / \mathbb{Z}$ avec $p$ parcourant l'ensemble des nombres premiers et $\mathbb{Z}[1 / p] / \mathbb{Z}=\operatorname{colim}_{v \in \mathbb{N}} \mathbb{Z} / p^{v} \mathbb{Z}$. Puisque $A$ et $\mathrm{La}_{t r}(A)$ sont compacts, le cas $M \otimes_{\mathbb{Z}} \mathbb{Q} / \mathbb{Z}$ découle de celui de $M \otimes_{\mathbb{Z}}(\mathbb{Z} / N \mathbb{Z})$ pour $N \in \mathbb{N}-\{0\}$. Autrement dit, on peut supposer que $M$ provient, par restriction des scalaires, d'un objet $M^{\prime} \in \mathbf{D A}^{\text {ét }}\left(S, \Lambda^{\prime}\right)$ pour $\Lambda^{\prime}=\Lambda \otimes \mathbb{Q}$ ou $\Lambda^{\prime}=\Lambda / N \Lambda$. Dans ces deux cas, on a un isomorphisme d'adjonction $\operatorname{hom}_{\mathbf{D A}^{\mathrm{et}}(S, \Lambda)}(A, M) \simeq$ $\operatorname{hom}_{\mathbf{D A}}{ }^{\mathrm{et}\left(S, \Lambda^{\prime}\right)}\left(A \otimes_{\Lambda} \Lambda^{\prime}, M\right)$ et de même pour $\mathbf{D M}^{\text {ét }}(S,-)$. Ceci permet de remplacer $\Lambda$ par $\Lambda^{\prime}$. Or, le cas de $\Lambda^{\prime}=\Lambda / N \Lambda$ découle de la Proposition B.2. Ceci termine la preuve du corollaire.

Dans le reste de l'annexe nous démontrerons le résultat suivant, ce qui permettra d'établir le Théorème B.1. Cette partie de la preuve est largement inspirée du travail de Cisinski-Deglise et nous ne prétendons pas à l'originalité. (Notre schéma de base sera appelé $B$ pour facilité la comparaison avec [8, Ann. B].)

Proposition B.4 - Soit B un schéma normal, japonais et de dimension de Krull finie. Soit $\Lambda$ une $\mathbb{Q}$-algèbre. Alors, le foncteur

$$
\mathrm{La}_{t r}: \mathbf{D A}^{\mathrm{e}} \mathrm{e}(B, \Lambda) \longrightarrow \mathbf{D M}^{\mathrm{e} t}(B, \Lambda)
$$

est une équivalence de catégories. 
Dans la suite, $B$ et $\Lambda$ seront comme dans l'énoncé de la Proposition B.4. Notons Nor/ $B$ la catégorie des $B$-schémas de type fini de source normale que l'on munit de la topologie étale. L'hypothèse que $B$ est japonais entraîne que le normalisé d'un $B$-schéma de type fini est encore de type fini. Ceci sera utilisé librement dans la suite.

La catégorie $\mathbf{C p l}(\mathbf{P S h}(\operatorname{Nor} / B, \Lambda))$ possède une structure de modèles projective ét-locale pour laquelle les équivalences faibles sont les morphismes de complexes induisant un isomorphisme sur les faisceaux étales associés aux préfaisceaux d'homologie. Comme d'habitude, on localise cette structure pour obtenir la structure projective $\left(\mathbb{A}^{1}\right.$, ét)-locale pour laquelle les flèches $\mathbb{A}_{X}^{1} \otimes$ $\Lambda[n] \longrightarrow X \otimes \Lambda[n]$ sont des équivalences faibles pour tout $X \in$ Nor $/ B$ et $n \in \mathbb{Z}$. La catégorie homotopique de la structure projective $\left(\mathbb{A}^{1}\right.$, ét)-locale sera notée $\mathbf{D}_{\mathbb{A}^{1} \text {, ét }}(\operatorname{Nor} / B, \Lambda)$.

L'inclusion $\mathfrak{i}_{B}: \mathrm{Sm} / B \hookrightarrow$ Nor $/ B$ induit un foncteur de Quillen à gauche

$$
\mathfrak{i}_{B}^{*}: \mathbf{C p l}(\mathbf{P S h}(\operatorname{Sm} / B, \Lambda)) \longrightarrow \mathbf{C p l}(\mathbf{P S h}(\operatorname{Nor} / B, \Lambda))
$$

pour les structures projectives ( $\mathbb{A}^{1}$, ét)-locales. (On se sert pour cela de [5, Th. 4.4.60] et on reprend l'argument de la preuve de [5, Th. 4.5.14].) On a le résultat suivant.

LeMme B.5 - Le foncteur $\mathrm{Li}_{B}^{*}: \mathbf{D A}^{\text {eff, ét }}(B, \Lambda) \longrightarrow \mathbf{D}_{\mathbb{A}^{1} \text {,ét }}(\mathrm{Nor} / B, \Lambda)$ est pleinement fidèle.

Demonstration Le lecteur vérifiera que la preuve de [8, Lem. B.2] est valable sans hypothèse sur les caractéristiques résiduelles de $B$.

Posons $T=\left(\mathbb{P}_{B}^{1}, \infty_{B}\right) \otimes \Lambda$ qu'on considère comme un préfaisceau sur $\mathrm{Sm} / B$ ou sur Nor $/ B$. Le foncteur (170) induit un foncteur de Quillen à gauche

$$
\mathfrak{i}_{B}^{*}: \operatorname{Spt}_{T}(\mathbf{C p l}(\mathbf{P S h}(\operatorname{Sm} / B, \Lambda))) \longrightarrow \operatorname{Spt}_{T}(\mathbf{C p l}(\mathbf{P S h}(\operatorname{Nor} / B, \Lambda)))
$$

pour les structures projectives ( $\mathbb{A}^{1}$, ét)-locales stables. (Pour des raisons techniques, nous travaillerons avec les $T$-spectres non symétriques; ceci est possible grâce à [5, Th. 4.3.79].) Notons $\mathbf{D}_{\mathbb{A}^{1} \text {,ét }}^{s t}(\operatorname{Nor} / B, \Lambda)$ la catégorie homotopique de $\operatorname{Spt}_{T}(\mathbf{C p l}(\mathbf{P S h}(\operatorname{Nor} / B, \Lambda)))$. On a le résultat suivant.

Corollaire B.6 - Le foncteur $\mathrm{Li}_{B}^{*}: \mathbf{D A}^{\mathrm{t}}(B, \Lambda) \longrightarrow \mathbf{D}_{\mathbb{A}^{1} \text {, ét }}^{\text {st }}(\mathrm{Nor} / B, \Lambda)$ est pleinement fidèle.

Demonstration Les deux catégories de modèles dans (170) vérifient [5, Hyp. 4.3.56]. On peut donc appliquer [5, Th. 4.3.61] pour obtenir des isomorphismes

$$
\begin{gathered}
\operatorname{hom}_{\mathbf{D A}^{\text {et }(B, \Lambda)}}\left(\operatorname{Sus}_{T}^{m}(X \otimes \Lambda), \operatorname{Sus}_{T}^{n}(Y \otimes \Lambda)[i]\right) \\
\simeq \operatorname{colim}_{r \geq \max (m, n)} \operatorname{hom}_{\mathbf{D A}^{\text {eff,ét }(B, \Lambda)}}\left(T^{\otimes r-m} \otimes(X \otimes \Lambda), T^{\otimes r-n} \otimes(Y \otimes \Lambda)[i]\right)
\end{gathered}
$$

(pour tout $X, Y \in \mathrm{Sm} / B$ et $(m, n, i) \in \mathbb{N} \times \mathbb{N} \times \mathbb{Z}$ ), ainsi que des isomorphismes analogues pour $\mathbf{D}_{\mathbb{A}^{1} \text {,ét }}^{s t}($ Nor $/ B, \Lambda)$. Puisque $\mathbf{D A}^{\text {ét }}(B, \Lambda)$ est compactement engendrée par les $\operatorname{Sus}_{T}^{m}(X \otimes \Lambda)$ pour $m \in \mathbb{N}$ et $X \in \mathrm{Sm} / B$, et que $\mathrm{Li}_{B}^{*}$ commute aux sommes infinies et préserve les objets compacts, le Lemme B.5 permet de conclure.

Soit $g: B^{\prime} \longrightarrow B$ un morphisme de type fini entre schémas normaux. On dispose d'un foncteur $g \circ-:$ Nor $/ B^{\prime} \longrightarrow$ Nor $/ B$ qui induit une adjonction de Quillen $\left(g_{\sharp}, g^{*}\right)$ relativement aux structures projectives $\left(\mathbb{A}^{1}\right.$, ét)-locales stables

$$
\operatorname{Spt}_{T}\left(\operatorname{Cpl}\left(\mathbf{P S h}\left(\operatorname{Nor} / B^{\prime}, \Lambda\right)\right)\right) \underset{g^{*}}{\stackrel{g_{\sharp}}{\rightleftarrows}} \operatorname{Spt}_{T}(\operatorname{Cpl}(\mathbf{P S h}(\operatorname{Nor} / B, \Lambda))) .
$$

L'argument de la première moitiée de la preuve de [8, Lem. B.2] s'applique aussi bien à $g^{*}$ pour montrer que ce dernier préserve les équivalences $\left(\mathbb{A}^{1}\right.$, ét)-locales stables. Il se dérive donc trivialement $: \mathrm{R} g^{*} \simeq g^{*}$. On a le résultat suivant. 
LEMME B.7 - Il existe un carré commutative à un isomorphisme canonique près

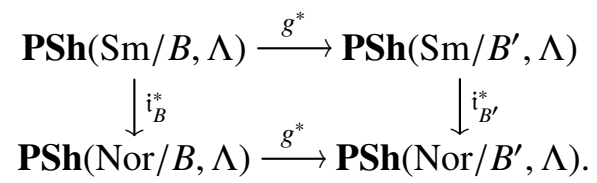

De plus, cet isomorphisme est compatible de la manière évidente à la composition des morphismes de schémas normaux.

Demonstration Le lecteur vérifiera que la preuve de [8, Lem. B.3] est valable sans hypothèse sur les caractéristiques résiduelles de $B$.

CoRollaire B.8 - Il existe un carré commutatif à un isomorphisme canonique près

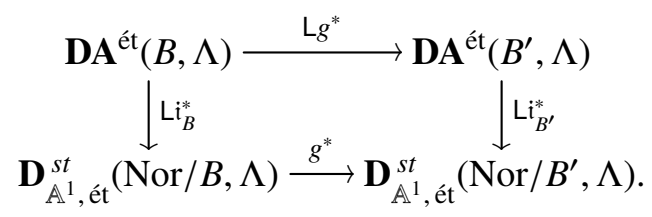

De plus, cet isomorphisme est compatible de la manière évidente avec la composition des morphismes de schémas normaux.

Demonstration Ceci découle immédiatement du Lemme B.7 et du fait que le foncteur de Quillen à droite $g^{*}$ de (172) se dérive trivialement.

Proposition B.9 - Soit $X$ un B-schéma de type fini et normal, et notons $f: X \longrightarrow B$ le morphisme structural. Pour tout $M \in \mathbf{D A}^{\mathrm{e}}{ }^{\mathrm{e}}(B, \Lambda)$, on a un isomorphisme canonique

$$
\operatorname{hom}_{\mathbf{D}_{\mathbb{A}^{1}, \text { et }}^{s t}(\operatorname{Nor} / B, \Lambda)}\left(\operatorname{Sus}_{T}^{0}(X \otimes \Lambda), \mathrm{Li}_{B}^{*} M\right) \simeq \operatorname{hom}_{\mathbf{D A}}{ }^{\text {et }(X, \Lambda)}\left(\Lambda_{X}, \mathrm{~L} f^{*} M\right) .
$$

Demonstration La preuve de [8, Prop. B.5] s'étend littéralement.

Rappelons que la topologie $f h$ (cf. [6, §2.2.1]) est la topologie de Grothendieck la moins fine sur Nor/ $B$ pour laquelle les familles suivantes sont couvrantes :

- la famille des inclusions des composantes connexes de $X \in$ Nor $/ B$,

- le singleton formé d'un morphisme fini surjectif entre $B$-schémas normaux et intègres.

Un préfaisceau $F$ sur Nor $/ B$ est un $f h$-faisceau si et seulement si il est additif (i.e., transforme un coproduit fini de $B$-schémas en un produit direct de $\Lambda$-modules) et pour tout revêtement pseudogaloisien de $B$-schémas normaux intègres $X^{\prime} \rightarrow X$, le morphisme $F(X) \rightarrow F\left(X^{\prime}\right)^{\text {aut }\left(X^{\prime} / X\right)}$ est inversible. De même, un complexe $K$ de préfaisceaux sur Nor/B est $f h$-local si et seulement si il est additif (à quasi-isomorphisme près) et si les morphismes $K(X) \longrightarrow \mathrm{R} \Gamma\left(\operatorname{aut}\left(X^{\prime} / X\right), F\left(X^{\prime}\right)\right)$ sont des isomorphismes dans $\mathbf{D}(\Lambda)$. Puisque $\Lambda$ est une $\mathbb{Q}$-algèbre, le foncteur $\Gamma\left(\operatorname{aut}\left(X^{\prime} / X\right),-\right)$ est exact et la condition précédente est équivalente à ce que le morphisme $K(X) \rightarrow K\left(X^{\prime}\right)^{\text {aut }\left(X^{\prime} / X\right)}$ soit un quasi-isomorphisme. Enfin, un objet $\mathbf{E} \in \mathbf{D}_{\mathbb{A}^{1} \text {, ét }}^{s t}(\operatorname{Nor} / B, \Lambda)$ sera dit $f h$-local, s'il en est ainsi des $\mathbf{F}_{m}$ (avec $m \in \mathbb{N}$ ) pour tout remplacement stablement ( $\mathbb{A}^{1}$, ét)-fibrant $\mathbf{F}$ de $\mathbf{E}$. Il est clair que la sous-catégorie pleine des objets $f h$-locaux est une sous-catégorie triangulée de $\mathbf{D}_{\mathbb{A}^{1} \text {, ét }}^{s t}(\operatorname{Nor} / B, \Lambda)$. Puisque $\Lambda$ est une $\mathbb{Q}$-algèbre, E est $f h$-local si et seulement si les homomorphismes

$$
\operatorname{hom}_{\mathbf{D}_{\mathbb{A}^{1}, \mathrm{et}}^{s t}(\operatorname{Nor} / B, \Lambda)}\left(\operatorname{Sus}_{T}^{m}(X \otimes \Lambda), \mathbf{E}\right) \longrightarrow \operatorname{hom}_{\mathbf{D}_{\mathbb{A}^{1}, \mathrm{et}}^{s t}(\operatorname{Nor} / B, \Lambda)}\left(\operatorname{Sus}_{T}^{m}\left(X^{\prime} \otimes \Lambda\right), \mathbf{E}\right)^{\operatorname{aut}\left(X^{\prime} / X\right)}
$$

sont inversibles pour tout $m \in \mathbb{N}$. Ceci permet de vérifier le résultat suivant.

Proposition B.10 - L'image de $\mathrm{Li}_{B}^{*}: \mathbf{D A}^{\mathrm{e}}(B, \Lambda) \longrightarrow \mathbf{D}_{\mathbb{A}^{1} \text {,ét }}^{s t}(\mathrm{Nor} / B, \Lambda)$ est contenue dans la sous-catégorie triangulée des objets fh-locaux.

Demonstration C'est ici que nous avons besoin de savoir que le 2-foncteur homotopique stable $\mathbf{D A}^{\text {ét }}(-, \Lambda): \operatorname{Sch} / B \longrightarrow \mathfrak{I} \Re$ est séparé (cf. le Théorème 3.9). Comme dans la preuve de $[\mathbf{8}$, Prop. B.6], on se ramène à montrer que id $\longrightarrow\left(r_{*} r^{*}\right)^{\text {aut( }\left(X^{\prime} / X\right)}$ est un isomorphisme pour $r: X^{\prime} \longrightarrow X$ un revêtement pseudo-galoisien entre schémas normaux et intègres de type fini sur $B$. 
On peut trouver une stratification $\mathcal{S}=\left(X_{i}\right)_{i \in I}$ de $X$ par des sous-schémas localement fermés, connexes et normaux, et telle que $r_{i}: X_{i}^{\prime}=\left(X^{\prime} \times_{X} X_{i}\right) \longrightarrow X_{i}$ est la composition d'un revêtement étale et d'un morphisme fini, surjectif et totalement inséparable. Par l'axiome de localité, il est alors suffisant de montrer que id $\longrightarrow\left(r_{i *} r_{i}^{*}\right)^{\text {aut }\left(X^{\prime} / X\right)}$ est inversible. Or, aut $\left(X^{\prime} / X\right)$ agit transitivement sur les composantes connexes de $X_{i}^{\prime}$. On en fixe une qu'on notera $X_{i 0}^{\prime}$ et on appellera $\operatorname{aut}_{0}\left(X^{\prime} / X\right)$ son stabilisateur et $r_{i 0}$ sa projection sur $X_{i}$. Il est alors suffisant de montrer que id $\longrightarrow\left(\left(r_{i 0}\right)_{*}\left(r_{i 0}\right)^{*}\right)^{\text {aut } 0\left(X^{\prime} / X\right)}=\left(\left(r_{i 0}\right)_{*}\left(r_{i 0}\right)^{*}\right)^{\text {aut }\left(X_{i 0}^{\prime} / X_{i}\right)}$ est inversible. Ceci découle maintenant de [5, Lem. 2.1.165].

On note $f$ hét la topologie sur Nor/B engendrée par la topologie étale et la topologie $f h$. Un préfaisceau sur Nor $/ B$ est un fhét-faisceau si et seulement si c'est un faisceau étale et un $f h$ faisceau. On a le résultat suivant (cf. [8, Lem. B.8]).

Lemme B.11 - Soit $F$ un fh-faisceau de $\Lambda$-modules sur Nor/B. Alors, le faisceau étale $\mathrm{a}_{\mathrm{e} t}(F)$ associé à $F$ est un fhét-faisceau.

Considérons maintenant la catégorie $\mathbf{S h}_{f h}(\operatorname{Nor} / B, \Lambda)$ des $f h$-faisceaux de $\Lambda$-modules sur Nor $/ B$. On dispose d'une structure de modèles fhét-locale sur $\mathbf{C p l}\left(\mathbf{S h}_{f h}(\operatorname{Nor} / B, \Lambda)\right)$ pour laquelle les équivalences faibles sont les morphismes de complexes de $f h$-faisceaux induisant des isomorphismes sur les fhét-faisceaux associés aux préfaisceaux d'homologie. Vu le Lemme B.11, il est loisible de qualifier cette structure de ét-locale et nous ferons cela dans la suite. On localise cette structure pour obtenir la structure projective $\left(\mathbb{A}^{1}\right.$, ét)-locale pour laquelle les flèches $\mathrm{a}_{f h}\left(\mathbb{A}_{X}^{1} \otimes \Lambda\right)[n] \longrightarrow \mathrm{a}_{f h}(X \otimes \Lambda)[n]$ sont des équivalences faibles pour tout $X \in$ Nor $/ B$ et $n \in \mathbb{Z}$. La catégorie homotopique de la structure projective $\left(\mathbb{A}^{1}\right.$, ét)-locale sera notée $\mathbf{D}_{\mathbb{A}^{1} \text {, ét }}^{f h}(\operatorname{Nor} / B, \Lambda)$. La variante stable, notée $\mathbf{D}_{\mathbb{A}^{1} \text {,ét }}^{f h, ~}(\operatorname{Nor} / B, \Lambda)$, est la catégorie homotopique de $\operatorname{Spt}_{T}\left(\mathbf{C p l}_{\mathbf{l}}\left(\mathbf{S h}_{f h}(\operatorname{Nor} / B, \Lambda)\right)\right)$ munie de sa structure projective $\left(\mathbb{A}^{1}\right.$, ét)-locale stable.

Proposition B.12 - Le foncteur $\mathrm{a}_{f h}: \mathbf{C p l}(\mathbf{P S h}(\operatorname{Nor} / B, \Lambda)) \longrightarrow \mathbf{C p l}\left(\mathbf{S h}_{f h}(\operatorname{Nor} / B, \Lambda)\right)$ est de Quillen à gauche. Il préserve les équivalences $\left(\mathbb{A}^{1}\right.$, ét)-locales et il en est de même de son adjoint à droite $\mathrm{O}_{\text {fh. }}$. L'énoncé analogue pour les catégories de T-spectres, munies de leurs structures projectives $\left(\mathbb{A}^{1}\right.$, ét)-locales stables, est également vrai.

Demonstration Le lecteur vérifiera que la preuve de [8, Prop. B.9] est valable sans hypothèse sur les caractéristiques résiduelles de $B$. Le passage aux catégories de spectres se fait sans difficulté. (Notons tout de même l'utilisation de [5, Prop. 4.2.74] pour vérifier que $\mathrm{o}_{f h}$ préserve les équivalences $\left(\mathbb{A}^{1}\right.$, ét)-locales stables.)

La Proposition B.12 fournit un foncteur $\mathrm{a}_{f h}=\mathrm{La}_{f h}: \mathbf{D}_{\mathbb{A}^{1} \text {, ét }}^{s t}(\operatorname{Nor} / B, \Lambda) \longrightarrow \mathbf{D}_{\mathbb{A}^{1} \text {, ét }}^{f h, s t}(\operatorname{Nor} / B, \Lambda)$. On a le résultat suivant.

Proposition B.13 - Le foncteur $\mathrm{a}_{f h} \circ \mathrm{Li}_{B}^{*}: \mathbf{D A}^{\text {ét }}(B, \Lambda) \longrightarrow \mathbf{D}_{\mathbb{A}^{1} \text {, ét }}^{f h}$ (Nor $\left./ B, \Lambda\right)$ est pleinement fidèle.

Demonstration Vu le Lemme B.5, il suffit de montrer que $\mathrm{Li}_{B}^{*} \rightarrow \mathrm{o}_{f h} \mathrm{a}_{f h} \mathrm{Li}_{B}^{*}$ est inversible. Soit $M \in \mathbf{D A}^{\text {ét }}(B, \Lambda)$. D'après la Proposition B.10, $\mathrm{Li}_{B}^{*} M$ est $f h$-local. Il en découle que le morphisme $\mathbf{E} \longrightarrow \mathrm{o}_{f h} \mathrm{a}_{f h} \mathbf{E}$ est un quasi-isomorphisme niveau par niveau pour tout remplacement projectivement stablement ( $\mathbb{A}^{1}$, ét)-fibrant $\mathbf{E}$ de $\mathrm{Li}_{B}^{*} M$. Ceci démontre la proposition.

Rappelons que $\operatorname{Cor}(B)$ désigne la catégorie ayant pour objets les $B$-schémas lisses et pour morphismes les correspondances finies. Les foncteurs contravariants additifs de $\operatorname{Cor}(B)$ à valeurs dans $\operatorname{Mod}(\Lambda)$ sont appelés les préfaisceaux avec transferts. Ils forment une catégorie abélienne notée $\operatorname{PST}(\operatorname{Sm} / B, \Lambda)$. D'après [46], on peut identifier $\operatorname{Cor}(B) \otimes \mathbb{Q}$ avec la sous-catégorie pleine de $\mathbf{S h}_{f h}(\operatorname{Nor} / B, \mathbb{Q})$ dont les objets sont les $f h$-faisceaux de la forme $\mathrm{a}_{f h}(X \otimes \mathbb{Q})$ avec $X$ un $B$-schéma lisse. Puisque $\Lambda$ est une $\mathbb{Q}$-algèbre, ceci permet de définir un foncteur

$$
\mathfrak{i}_{B, t r}^{*}: \operatorname{PST}(\operatorname{Sm} / B, \Lambda) \longrightarrow \mathbf{S h}_{f h}(\operatorname{Nor} / B, \Lambda)
$$


adjoint à gauche du foncteur $\mathfrak{i}_{B, t r *}$ qui à un $f h$-faisceau $F$ associe le préfaisceau avec transferts : $X \in \mathrm{Sm} / B \leadsto \operatorname{hom}\left(\mathrm{a}_{f h}(X \otimes \mathbb{Z}), F\right)$. On a le fait suivant.

Lemme B.14 - Le foncteur $\mathrm{i}_{B, t r}^{*}: \mathbf{C p l}(\mathbf{P S T}(\operatorname{Sm} / B, \Lambda)) \longrightarrow \mathbf{C p l}\left(\mathbf{S h}_{f h}(\operatorname{Nor} / B, \Lambda)\right)$ est de Quillen à gauche relativement aux structures $\left(\mathbb{A}^{1}\right.$, ét)-locales.

Demonstration Le lecteur vérifiera que la preuve de [8, Lem. B.11] est valable sans hypothèse sur les caractéristiques résiduelles de $B$.

LeMme B.15 - Le foncteur $\mathrm{Li}_{B, t r}^{*}: \mathbf{D M}^{\mathrm{e}^{\mathrm{t}}}(B, \Lambda) \longrightarrow \mathbf{D}_{\mathbb{A}^{1} \text {, ét }}^{\text {fht }}(\mathrm{Nor} / B, \Lambda)$ est pleinement fidèle.

Demonstration Comme pour [8, Lem. B.12], il s'agit d'adapter la preuve de [8, Lem. B.2]. Ensuite on raisonne comme dans la preuve du Corollaire B.6.

Expliquons maintenant comment terminer la preuve de la Proposition B.4. Il est facile de voir qu'on a un carré commutatif à un isomorphisme canonique près

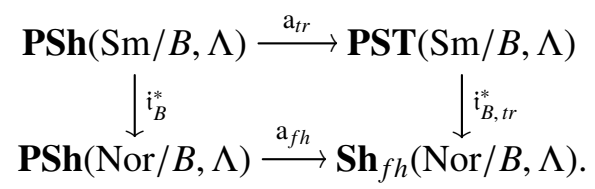

(En fait, la commutativité se vérifie plus facilement sur les adjoints à droite.) En passant aux catégories de $T$-spectres de complexes et en inversant ensuite les équivalences ( $\mathbb{A}^{1}$, ét)-locales stables, on obtient un carré commutatif à un isomorphisme canonique près

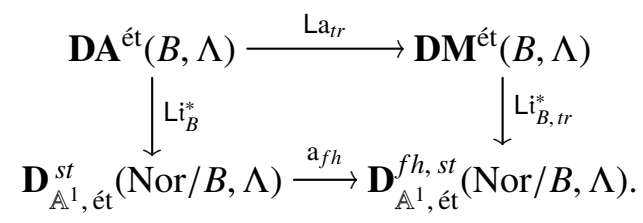

La Proposition B.13 et le Lemme B.15 entrainent alors que $\operatorname{La}_{t r}: \mathbf{D A}^{\text {ét }}(B, \Lambda) \longrightarrow \mathbf{D M}^{\text {ét }}(B, \Lambda)$ est pleinement fidèle. Or, il commute aux sommes infinies et son image contient des générateurs compacts de $\mathbf{D} \mathbf{M}^{\text {eff, ét }}(B, \Lambda)$. C'est donc une équivalence de catégories !

\section{Annexe C}

\section{Vérification de la condition $\left(\mathrm{SS}_{p}\right)$ : deuxième méthode}

Dans cet annexe, nous donnons une vérification directe de la condition $\left(\mathbf{S S}_{p}\right)$, introduite à la Section 1, dans le cas du 2-foncteur homotopique stable $\mathbf{D A} \mathbf{A}^{\text {ét }}(-, \Lambda) .{ }^{(11)}$ L'argument est plus court que celui présenté dans la Section 2 et a l'avantage de ne pas nécessiter que 2 soit inversible dans $\Lambda$. En revanche, il est moins «self-contained » puisqu'il repose sur des résultats de F. Morel sur les groupes d'homotopie des sphères motiviques $[\mathbf{3 2}, \mathbf{3 3}, \mathbf{3 6}]$.

Theoreme C.1 - Soit $S$ un schéma de base. Alors, la condition $\left(\mathbf{S S}_{p}\right)$ est satisfaite dans le 2-foncteur homotopique stable $\mathbf{D A}^{\mathrm{e} t}(-, \Lambda): \mathrm{Sch} / S \longrightarrow \mathfrak{I} \mathfrak{R}$ pour tout nombre premier $p$.

Avant de donner la preuve du Théorème C.1, nous établissons un point technique qui n'a rien d'étonnant mais pour lequel nous ne disposons pas de référence. On rappelle que $\operatorname{Sus}_{T, \Sigma}^{0}$ désigne le 0 -ième foncteur de suspension infinie comme dans [5, Déf. 4.3.10].

Lemme C.2 - Soit $S$ un schéma de base, et soit $f: Y \longrightarrow X$ un morphisme de $S$-schémas quasi-projectifs et lisses. Notons $q: X \longrightarrow S$ le morphisme structural. Alors, le morphisme

$$
(q \circ f) !(q \circ f) ! \Lambda_{S}(0) \simeq q ! f ! f^{!} q^{!} \Lambda_{S}(0) \stackrel{\delta}{\longrightarrow} q ! q^{!} \Lambda_{S}(0)
$$

11. Nous avons trouvé ce moyen direct pour vérifier la condition $\left(\mathbf{S S}_{p}\right)$ à la fin de mai 2013, bien après l'acceptation de l'article en novembre 2012. Afin de minimiser les changements nous présentons l'argument en annexe. 
s'identifie canoniquement au morphisme

$$
\operatorname{Sus}_{T, \Sigma}^{0}(f): \operatorname{Sus}_{T, \Sigma}^{0}(Y \otimes \Lambda) \longrightarrow \operatorname{Sus}_{T, \Sigma}^{0}(X \otimes \Lambda) .
$$

Demonstration Cet énoncé est non trivial car la construction des «morphismes de connexions » pour les 2-foncteurs $H^{!}$et $H_{\text {! }}$ est compliquée (voir [4, §1.6]). Une façon économe de s'en sortir est de se ramener à [4, Prop. 2.3.53] en utilisant le bifoncteur « homomorphismes internes ».

Vu que $q^{!}=\operatorname{Th}\left(\Omega_{q}\right) \circ q^{*}$ et $q_{!}=q_{\sharp} \circ \mathrm{Th}^{-1}\left(\Omega_{q}\right)$, on a les identifications :

$$
q ! q^{!} \Lambda_{S}(0)=q_{\sharp} q^{*} \Lambda_{S}(0)=q_{\sharp} \Lambda_{X}(0)=\operatorname{Sus}_{T, \Sigma}^{0}(X \otimes \Lambda) .
$$

Il en est de même pour $q \circ f$. Ainsi, on dispose de deux morphismes de $\operatorname{Sus}_{T, \Sigma}^{0}(Y \otimes \Lambda)$ vers $\operatorname{Sus}_{T, \Sigma}^{0}(X \otimes \Lambda)$ :

- le premier, noté $\operatorname{Sus}_{T, \Sigma}^{0}(f)$, est induit directement de $f$;

- le second, qu'on note $\delta(f)$, est déduit de la composition de (175) et des identifications cidessus.

Il s'agit de montrer que $\delta(f)=\operatorname{Sus}_{T, \Sigma}^{0}(f)$. Pour cela, il suffit de montrer que le foncteur contrava-

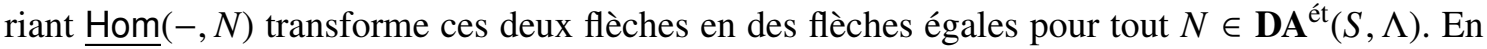
effet, puisque

$$
\mathrm{R} \Gamma\left(S, \operatorname{REv}_{0} \underline{\operatorname{Hom}}(-, N)\right)=\operatorname{hom}_{\mathbf{D A}^{\mathrm{et}}(S, \Lambda)}(-, N),
$$

le lemme de Yoneda permettrait de conclure.

Donnons-nous donc un motif $N \in \mathbf{D A}^{\text {ét }}(S, \Lambda)$. D'après [4, Prop. 2.3.53] et le Sous-lemme C.3 ci-dessous, on dispose d'un diagramme commutatif

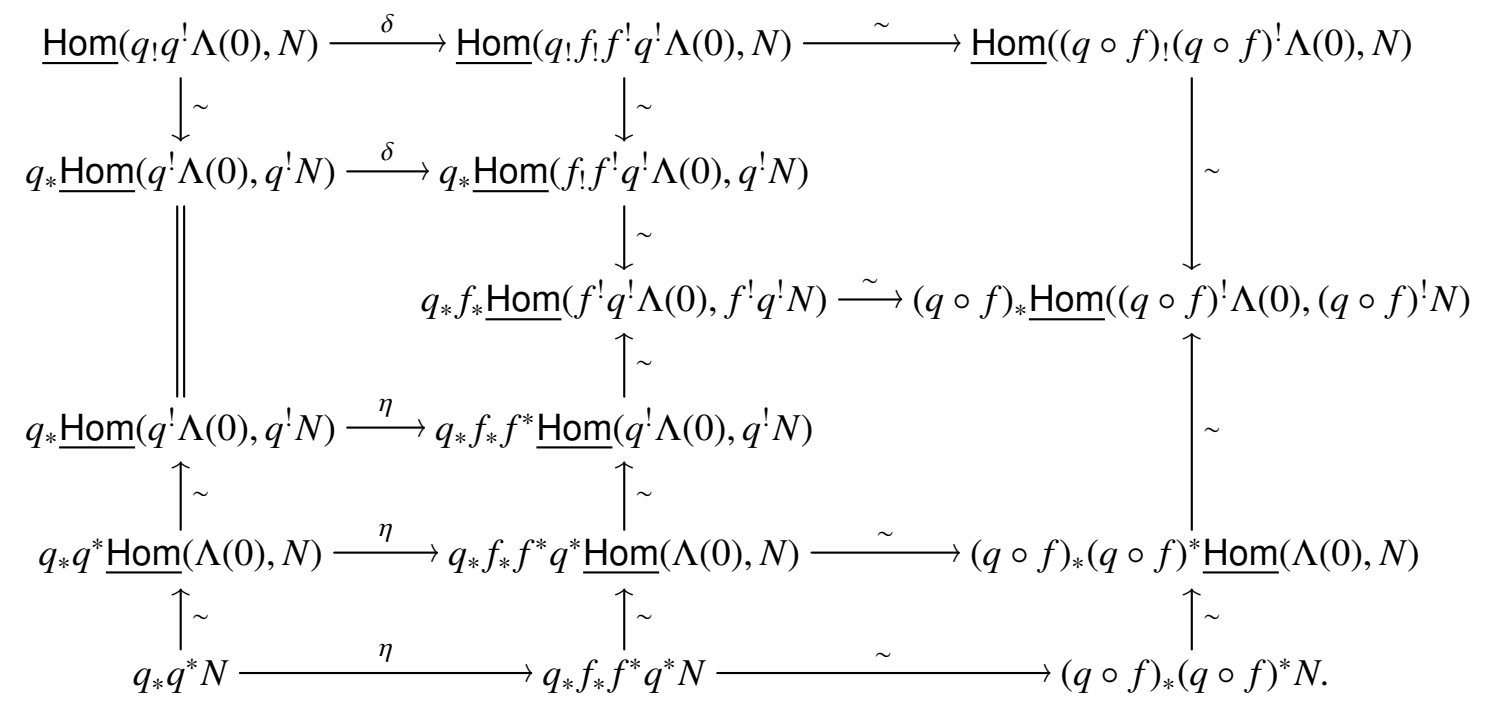

(Le Sous-lemme C.3 assure la commutation du grand carré de gauche et fournit aussi les flèches verticales partant de la deuxième ligne d'en bas.)

Par ailleurs, on a des identifications canoniques

$$
q_{*} q^{*} N \simeq \operatorname{Rhom}(X, N) \quad \text { et } \quad(q \circ f)_{*}(q \circ f)^{*} N \simeq \operatorname{Rom}(Y, N) .
$$

(Ci-dessus, Rhom est le foncteur dérivé de l'extension aux $T$-spectres symétriques du foncteur hom rappelé au début de la Section 3.) De plus, modulo ces identifications et celles d'avant, l'isomorphisme $\underline{\operatorname{Hom}}\left(q ! q^{!} \Lambda(0), N\right) \simeq q_{*} q^{*} N$, composé des isomorphismes verticaux à gauche dans le diagramme ci-dessus, est donné par l'isomorphisme canonique

$$
\underline{\operatorname{Hom}}\left(\operatorname{Sus}_{T, \Sigma}^{0}(X \otimes \Lambda), N\right) \simeq \operatorname{Rhom}(X, N) .
$$


Il en est de même pour $q \circ f$ et $Y$ au lieu de $q$ et $X$. Le diagramme commutatif ci-dessus montre alors que le morphisme

$$
\underline{\operatorname{Hom}}(\delta(f), N): \underline{\operatorname{Hom}}\left(\operatorname{Sus}_{T, \Sigma}^{0}(X \otimes \Lambda), N\right) \longrightarrow \underline{\operatorname{Hom}}\left(\operatorname{Sus}_{T, \Sigma}^{0}(Y \otimes \Lambda), N\right)
$$

correspond au morphisme $\operatorname{Rhom}(f, N): \underline{\operatorname{hom}}(X, N) \longrightarrow \underline{\operatorname{hom}}(Y, N)$. Or, par construction, il en est

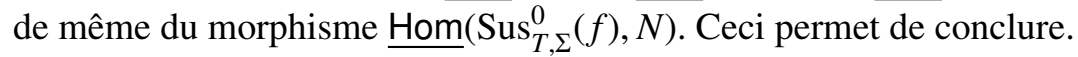

Sous-lemme C.3 - Soit $f: Y \longrightarrow X$ un morphisme quasi-projectif. Il existe alors une transformation binaturelle en $M, N \in \mathbf{D A}^{\text {ét }}(X, \Lambda)$ :

$$
f^{*} \underline{\operatorname{Hom}}(M, N) \longrightarrow \underline{\operatorname{Hom}}\left(f^{!} M, f^{!} N\right) .
$$

De plus, le carré suivant est commutatif

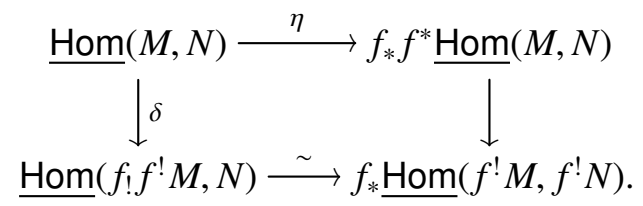

Enfin, si f est lisse, (176) est inversible et elle est donnée par la composition des isomorphismes

$$
f^{*} \underline{\operatorname{Hom}}(M, N) \simeq \underline{\operatorname{Hom}}\left(f^{*} M, f^{*} N\right) \simeq \underline{\operatorname{Hom}}\left(\operatorname{Th}\left(\Omega_{f}\right) f^{*} M, \operatorname{Th}\left(\Omega_{f}\right) f^{*} N\right)=\underline{\operatorname{Hom}}\left(f^{!} M, f^{!} N\right) .
$$

Demonstration Le morphisme en question est déduit via l'adjonction $\left(f^{*}, f_{*}\right)$ de la composition de

$$
\underline{\operatorname{Hom}}(M, N) \stackrel{\delta}{\longrightarrow} \underline{\operatorname{Hom}}\left(f_{!} f^{!} M, N\right) \simeq f_{*} \underline{\operatorname{Hom}}\left(f^{!} M, f^{!} N\right)
$$

(L'isomorphisme utilisé ci-dessus est celui de [4, Prop. 2.3.53].) La commutation du carré est immédiate. Il en est de même de la dernière assertion.

Revenons maintenant à la preuve du Théorème C.1. Avec les notations utilisées dans la formulation de la condition $\left(\mathbf{S S}_{p}\right)$, on a la réduction suivante.

Lemme C.4 - Pour démontrer le Théorème C.1, il suffit de montrer, que pour tout nombre premier $p$, il existe une extension finie $k / \mathbb{F}_{p}$ telle que, pour tout $n \in \mathbb{N}$, l'endomorphisme

$$
F_{n}: \operatorname{Sus}_{T, \Sigma}^{0}\left(\left(G_{k}, 1\right) \otimes \Lambda\right) \longrightarrow \operatorname{Sus}_{T, \Sigma}^{0}\left(\left(G m_{k}, 1\right) \otimes \Lambda\right)
$$

est un isomorphisme dans $\mathbf{D} \mathbf{A}^{\text {ét }}(k, \Lambda)$.

Demonstration Vu le Lemme 3.4, on peut supposer que (177) est un isomorphisme pour $k=\mathbb{F}_{p}$. Il s'ensuit que le morphisme

$$
F_{n}: \operatorname{Sus}_{T, \Sigma}^{0}\left(\mathbb{G m}_{\mathbb{F}_{p}} \otimes \Lambda\right) \longrightarrow \operatorname{Sus}_{T, \Sigma}^{0}\left(\mathbb{G}_{\mathbb{F}_{p}} \otimes \Lambda\right)
$$

est aussi un isomorphisme. En appliquant le foncteur «image inverse » suivant le morphisme structural $S /(p) \longrightarrow \operatorname{Spec}\left(\mathbb{F}_{p}\right)$, on déduit que le morphisme

$$
F_{n}: \operatorname{Sus}_{T, \Sigma}^{0}\left(\mathbb{G m}_{S /(p)} \otimes \Lambda\right) \longrightarrow \operatorname{Sus}_{T, \Sigma}^{0}\left(\mathbb{G m}_{S /(p)} \otimes \Lambda\right)
$$

est un isomorphisme dans $\mathbf{D} \mathbf{A}^{\text {ét }}(S /(p), \Lambda)$. On divise le reste de la preuve en deux étapes.

Étape 1 : Pour $M \in \mathbf{D A}^{\text {ét }}(S /(p), \Lambda)$, on dispose d'un diagramme commutatif

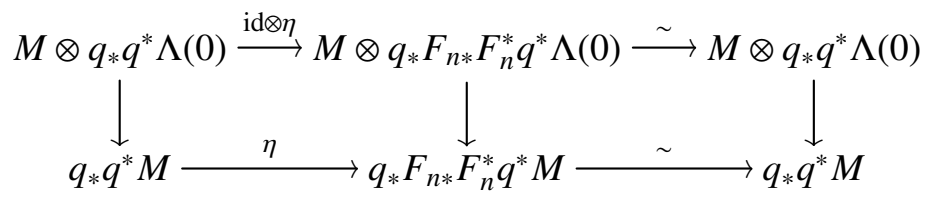

où les flèches verticales sont déduites des morphismes de coprojection. Ces flèches verticales sont des isomorphismes. (Remarquer que les deux membres sont canoniquements isomorphes à $M \oplus$ 
$M(-1)[-1]$.) Il s'ensuit que la transformation naturelle $\eta: q_{*} q^{*} \longrightarrow q_{*} F_{n *} F_{n}^{*} q^{*}$ est inversible si et seulement si le morphisme

$$
\eta: q_{*} q^{*} \Lambda_{S /(p)}(0) \longrightarrow q_{*} F_{n *} F_{n}^{*} q^{*} \Lambda_{S /(p)}(0)
$$

est inversible. Pour conclure, il reste à remarquer que le morphisme (180) s'obtient du morphisme (179) en appliquant $\underline{\mathrm{Hom}}\left(-, \Lambda_{S /(p)}(0)\right)$.

Étape 2 : Dualement, en utilisant [4, Th. 2.3.38 et Cor. 2.3.39], on dispose d'un diagramme commutatif

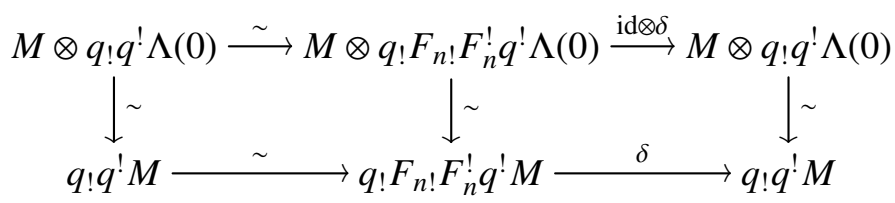

où les flèches verticales sont inversibles. L'isomorphisme $M \otimes q ! q ! \Lambda(0) \simeq q ! q ! M$ est la composition de

$$
M \otimes q ! q^{!} \Lambda(0) \longrightarrow q !\left(q^{*} M \otimes q^{!} \Lambda(0)\right) \longrightarrow q ! q^{!} M
$$

la première flèche est inversible par [4, Th. 2.3.40] et la seconde flèche est inversible par construction (voir [4, Déf. 2.3.27]). Ainsi, comme avant, la transformation naturelle $\delta: q ! F_{n !} F_{n}^{!} q^{!} \longrightarrow q ! q^{!}$ est inversible si et seulement si le morphisme

$$
\delta: q ! F_{n !} F_{n}^{!} q ! \Lambda(0) \longrightarrow q ! q ! \Lambda(0)
$$

est inversible. Or, d'après le Lemme C.2, le morphisme (181) s'identifie au morphisme (179).

À partir de maintenant, on fixe un corps $k$. (Nous sommes intéressés par le cas où $k$ est un corps fini et en particulier parfait, mais pour les besoin de la preuve, nous sommes amenés à considérer des corps plus généraux, notamment $k(t)$.) Notons $e_{m}: \mathbb{G}_{k} \longrightarrow \mathbb{G}_{k}$ le morphisme d'élévation à la puissance $m \in \mathbb{Z}$. (Lorsque $k$ est une extension de $\mathbb{F}_{p}$ on a $F_{n}=e_{p^{n}}$.) Par construction, on a un isomorphisme $\operatorname{Sus}_{T, \Sigma}^{0}\left(\left(\mathbb{G m}_{k}, 1\right) \otimes \Lambda\right) \simeq \Lambda_{k}(1)[1]$ dans $\mathbf{D A}^{\text {ét }}(k, \Lambda)$. Ainsi, l'endomorphisme $e_{m}$ définit un élément

$$
\left[e_{m}\right] \in \operatorname{end}_{\mathbf{D A}^{(\mathrm{t}}(k, \Lambda)}(\Lambda(1)[1]) \simeq \operatorname{end}_{\mathbf{D A}^{(\mathrm{t} t}(k, \Lambda)}(\Lambda(0)) .
$$

Le Théorème C.1 est maintenant une conséquence directe des Lemmes 3.10 et C.4, et du résultat suivant.

Proposition C.5 - Supposons que - 1 est un carré dans $k$. L'élément $\left[e_{m}\right]$ est égal à $m \cdot \mathrm{id}_{\Lambda(0)}$.

Remarque C.6 - Dans l'énoncé de la Proposition C.5, l'hypothèse $-1 \in\left(k^{\times}\right)^{2}$ est superflue. En effet, on peut obtenir l'égalité $\left[e_{m}\right]=m \cdot \operatorname{id}_{\Lambda(0)}$ dans end ${ }_{\mathbf{D A}^{\text {et }}(k, \Lambda)}(\Lambda(0))$ en toute généralité comme conséquence du Théorème B. 1 et du calcul de la cohomologie motivique en bidegré $(0,0)$. Toutefois, pour éviter un cercle vicieux, nous devons fournir une preuve directe de la Proposition C.5, i.e., qui ne repose pas sur le Théorème B.1. En effet, la preuve du Théorème B.1 repose sur le théorème de rigidité relative (Théorème 4.1) qui lui-même repose sur la vérification de la condition $\left(\mathbf{S S}_{p}\right)$ et donc en particulier sur la Proposition C.5 (du mois si 2 n'est pas inversible dans $\Lambda$ ).

Pour démontrer la Proposition C.5, nous aurons besoin d'une digression $\mathbb{A}^{1}$-homotopique. ${ }^{(12)}$ Introduisons d'abord quelques notations.

Considérons les catégories $\mathbb{A}^{1}$-homotopiques $\mathbf{S} H^{\text {eff }}(k)$ et $\mathbf{S H}(k)$, aka., les catégories des motifs à coefficients dans la catégorie des $S^{1}$-spectres symétriques $\mathfrak{M}=\mathbf{S p t}_{\mathrm{S}^{1}}^{\Sigma}\left(\Delta^{\mathrm{op}} \mathcal{E} n s_{\bullet}\right.$ ) (voir le début de l'Annexe A). Pour $p \in \mathbb{N}$ et $q \in \mathbb{Z}$, on pose $\mathrm{S}^{p, q}=\left(\mathbb{G}_{k}, 1\right)^{\wedge p} \otimes \mathrm{S}^{q-p}$ avec $\mathrm{S}^{q-p}$ la sphère simpliciale stable de dimension $q-p$; ce sont des objets de $\mathbf{S H}{ }^{\text {eff }}(k)$. On note encore $\mathrm{S}^{p, q}$ le spectre $\operatorname{Sus}_{T, \Sigma}^{0}\left(\mathrm{~S}^{p, q}\right)$ et on étend les $\mathrm{S}^{p, q}$ aux $p<0$ de la manière usuelle; ces sont des objets de $\mathbf{S H}(k)$. (Si l'on désigne par $\mathbb{1}$ l'objet unité de $\mathbf{S H}(k)$, alors $\mathbf{S}^{p, q}=\mathbb{1}(p)[q]$.) Nous aurons besoin du résultat suivant de F. Morel.

12. En fait, vu le contexte, il aurait été plus naturel de faire une digression $\mathbb{A}^{1}$-homologique. Toutefois, pour faciliter les références aux travaux de $\mathrm{F}$. Morel, il sera plus pratique de parler de $\mathbb{A}^{1}$-homotopie au lieu de $\mathbb{A}^{1}$-homologie. 
Theoreme C.7 - Pour tout $n \in \mathbb{Z}$, on a un isomorphisme canonique

$$
\operatorname{hom}_{\mathbf{S H}(k)}(\mathbb{1}, \mathbb{1}(n)[n]) \simeq \mathrm{K}_{n}^{M W}(k)
$$

où $\mathrm{K}_{n}^{M W}(k)$ est le groupe de K-théorie de Milnor-Witt.

Ce théorème de F. Morel est énoncé dans [33, Th. 6.2.2] (voir aussi [32, Th. 6.4.1]) sous l'hypothèse que le corps $k$ est parfait, ce qui est malheureusement trop restrictif pour nos besoins. Heureusement, en utilisant des résultats de [36], il est possible d'obtenir ce théorème en toute généralité (voir le Corollaire C.9(b) ci-dessous). Expliquons comment.

Pour $M \in \mathbf{S H}^{\mathrm{eff}}(k)$ et $i \in \mathbb{Z}$, on note $\pi_{i}^{\mathbb{A}^{1}}(M)$ le faisceau Nisnevich associé au préfaisceau $X \in$ $\mathrm{Sm} / k \rightsquigarrow \operatorname{hom}_{\mathbf{S H}^{\mathrm{eff}}(k)}\left(X \otimes \mathrm{S}^{i}, M\right)$. D'après [34, Lem. 6.4.4], c'est un faisceau Nisnevich strictement invariant par homotopie. Le résultat suivant est bien connu.

Lemme C.8 - Pour $M \in \mathbf{S H}^{\mathrm{eff}}(k)$ et $n \geq 0$, on a

$$
\operatorname{hom}_{\mathbf{S H}^{\mathrm{eff}}(k)}\left(\mathrm{S}^{n, n}, M\right) \simeq\left(\pi_{0}^{\mathbb{A}^{1}}(M)\right)_{-n}(k)
$$

où $(-)_{-n}$ désigne la n-ième contraction. ${ }^{(13)}$

Demonstration Par la suite spectrale induite de la $t$-structure homotopique $\operatorname{sur} \mathbf{S} \mathbf{H}^{\mathrm{eff}}(k)$ (voir [34]), il suffit de montrer que

$$
\mathrm{H}_{\mathrm{Nis}}^{j}\left(\left(\mathrm{Gm}_{k}, 1\right)^{\wedge n}, \pi_{i}^{\mathbb{A}^{1}}(M)\right)=\left\{\begin{array}{ccc}
\left(\pi_{i}^{\mathbb{A}^{1}}(M)\right)_{-n}(k) & \text { si } & j=0 \\
0 & \text { si } & j \neq 0 .
\end{array}\right.
$$

Il sera donc plus général de montrer que

$$
\operatorname{Rhom}\left(\left(\mathrm{Gm}_{k}, 1\right)^{\wedge n}, F[0]\right) \simeq(F)_{-n}[0]
$$

pour tout faisceau Nisnevich strictement invariant par homotopie $F$. Ceci permet d'employer une récurrence pour se ramener au cas $n=1$.

Il reste donc à montrer que $G^{j}=\mathrm{R}^{j} \underline{\underline{h o m}}\left(\left(\mathrm{Gm}_{k}, 1\right), F\right)$ est nul pour $j \geq 1$. D'après [34, Th. 6.2.7], appliqué à $\underline{\operatorname{Rhom}}\left(\left(\mathrm{Gm}_{k}, 1\right), F\right)$, les faisceaux $G^{j}$ sont strictement invariants par homotopie. D'après [34, Lem. 6.4.4], si $U \subset X$ est un ouvert dense d'un $k$-schéma lisse $X$, le morphisme de restriction $G^{j}(X) \longrightarrow G^{j}(U)$ est injectif. Ainsi, pour démontrer que $G^{j}$ est nul, il suffit de montrer que $G^{j}(K)=0$ pour toute extension ind-lisse $K / k$. Étant donné que $\left(G^{-} \mathrm{m}_{K}, 1\right) \otimes \mathbb{Z} \simeq\left(\mathbb{P}_{K}^{1}, \infty\right) \otimes \mathbb{Z}[-1]$ dans $\mathbf{D A} \mathbf{e f f}^{\text {eff }}(K, \mathbb{Z})$, on trouve que

$$
G^{j}(K)=\mathrm{H}_{\mathrm{Nis}}^{j}\left(\left(\mathrm{Gm}_{K}, 1\right), F\right) \simeq \mathrm{H}_{\mathrm{Nis}}^{j+1}\left(\left(\mathbb{P}_{K}^{1}, \infty\right), F\right) .
$$

Or, le membre de droite est un facteur direct de $\mathrm{H}^{j+1}\left(\mathbb{P}_{K}^{1}, F\right)$ qui s'annule, pour $j \geq 1$, car $\mathbb{P}_{K}^{1}$ est de dimension cohomologique 1 relativement à la topologie Nisnevich. ${ }^{(14)}$

Lorsque $p \geq 1$, [36, Cor. 6.43] fournit un isomorphisme de faisceaux Nisnevich strictement invariants par homotopie

$$
\pi_{0}^{\mathbb{A}^{1}}\left(\mathrm{~S}^{p, p}\right) \simeq \underline{\mathbf{K}}_{p}^{M W}
$$

avec $\underline{\mathbf{K}}_{p}^{M W}$ le faisceau de $K$-théorie de Milnor-Witt non ramifiée (voir [36, §3.2]). À vrai dire, dans [36], le corps de base est supposé parfait; mais puisque $\mathrm{S}^{p, p}$ et $\underline{\mathbf{K}}_{p}^{M W}$ proviennent par « image inverse » du sous-corps premier $k_{0} \subset k$, il est facile de déduire l'isomorphisme (182) pour $k$ de celui pour $k_{0}$.

La partie (b) de l'énoncé suivant est une reformulation du Théorème C.7.

\section{Corollaire C.9 -}

13. Rappelons que, pour un préfaisceau de groupes abéliens $F$ sur Sm $/ k$, le préfaisceau $(F)_{-1}$ est défini comme étant le noyau du morphisme $1^{*}: \underline{\operatorname{hom}}\left(\mathbb{G m}_{k}, F\right) \longrightarrow F$ induit par la section unité de $\mathbb{G m}_{k}$. Autrement dit, on a $(F)_{-1}=$ $\underline{\operatorname{hom}}((G \mathrm{Gm}, 1), F)$. Pour $n \geq 0$, le préfaisceau $(F)_{-n}$ est défini par récurrence à l'aide de la formule $(F)_{-n}=\left((F)_{-(n-1)}\right)_{-1}$ (et la convention que $\left.F_{-0}=F\right)$. On a encore $(F)_{-n}=\operatorname{hom}\left((\mathbb{G m}, 1)^{\wedge n}, F\right)$.

14. J'ai appris cette preuve de l'annulation de $\mathrm{H}_{\mathrm{Nis}}^{j}\left(\mathrm{G} \mathrm{m}_{K}, F\right)$, pour $j \geq 1$, lors d'une discussion avec F. Morel qui avait attribué ladite preuve à M. Hopkins. 
(a) Pour $n \geq 0$ et $p \geq 1$, on a un isomorphisme canonique $\operatorname{hom}_{\mathbf{S H}^{\mathrm{eff}}(k)}\left(\mathrm{S}^{n, n}, \mathrm{~S}^{p, p}\right) \simeq \underline{\mathbf{K}}_{p-n}^{M W}(k)$.

(b) Pour tout $n, p \in \mathbb{Z}$, on a un isomorphisme canonique $\operatorname{hom}_{\mathbf{S H}(k)}\left(\mathrm{S}^{n, n}, \mathrm{~S}^{p, p}\right) \simeq \underline{\mathbf{K}}_{p-n}^{M W}(k)$.

Demonstration La partie (a) découle aussitôt du Lemme C.8, de l'isomorphisme (182) et du fait que $\left(\underline{\mathbf{K}}_{p}^{M W}\right)_{-n} \simeq \underline{\mathbf{K}}_{p-n}^{M W}$ (ce qui doit découler de [36, §3.2] à en croire la preuve de [36, Cor. 6.43]). La partie (b) découle de (a) et du fait que le groupe hom $\operatorname{SH}_{(k)}\left(\mathrm{S}^{n, n}, \mathrm{~S}^{p, p}\right)$ est la colimite suivant $r \geq \max (0,-p,-n)$ des groupes $\operatorname{hom}_{\mathbf{S H}^{\text {eff }}(k)}\left(\mathrm{S}^{n+r, n+r}, \mathrm{~S}^{p+r, p+r}\right)$. (Ceci découle par exemple de [5, Th. 4.3.61].)

On est maintenant en mesure de démontrer la Proposition C.5. L'endomorphisme $e_{m}$ de $\mathbb{G}_{k}$ induit un élément

$$
\left\{e_{m}\right\} \in \operatorname{end}_{\mathbf{S H}(k)}\left(\mathrm{S}^{1,1}\right) \simeq \operatorname{end}_{\mathbf{S H}(k)}\left(\mathrm{S}^{0,0}\right) .
$$

La classe $\left[e_{m}\right]$ est égale à l'image de $\left\{e_{m}\right\}$ par le foncteur composé

$$
\mathbf{S H}(k) \longrightarrow \mathbf{S H}^{\text {ét }}(k) \longrightarrow \mathbf{D A}^{\text {ét }}(k, \Lambda)
$$

(cf. (164)). Il suffit donc de montrer que $\left\{e_{m}\right\}=m \cdot \operatorname{id}_{\mathrm{S}^{1,1}}$.

Soit $l / k$ une extension ind-lisse. Un élément $a \in l^{\times}$définit une classe

$$
[a] \in \operatorname{hom}_{\mathbf{S H}(l)}\left(\mathrm{S}^{0,0}, \mathrm{~S}^{1,1}\right)
$$

qui correspond, via l'isomorphisme du Corollaire C.9(b), au symbole $[a] \in \mathrm{K}_{1}^{M W}(l)$ associé à $a$ (voir [36, Def. 3.1]). L'élément $\left\{e_{m}\right\} \in \operatorname{end}_{\mathbf{S H}(k)}\left(S^{0,0}\right)$ agit sur $[a]$ par la formule $\left\{e_{m}\right\} \cdot[a]=\left[a^{m}\right]$. Étant donné que l'isomorphisme du Corollaire C.9(b) est compatible au changement de corps de base et à la multiplication, il s'ensuit que $\left\{e_{m}\right\}$, vu comme élément de $\mathrm{K}_{0}^{M W}(k)$, agit sur $\mathrm{K}_{1}^{M W}(l)$ par la même formule.

Considérons maintenant l'élément $m_{\epsilon} \in \mathrm{K}_{0}^{M W}(k)$ donné par

$$
m_{\epsilon}=\sum_{i=1}^{n}\left\langle(-1)^{i-1}\right\rangle,
$$

où, pour $u \in k^{\times},\langle u\rangle=1+\eta[u]$. Pour terminer la preuve de la Proposition C.5, il suffit de vérifier que $\left\{e_{m}\right\}=m_{\epsilon}$ dans $\mathrm{K}_{0}^{M W}(k)$. En effet, puisque -1 est un carré dans $k$, on a $\langle-1\rangle=\langle 1\rangle=1$ (voir [36, Lem. 3.9]) ce qui assure que $m_{\epsilon}=m$.

Par [36, Lem. 3.14] et puisque $\mathrm{K}_{1}^{M W}(l)$ est engendré (comme groupe abélien) par les classes $[a]$ des $a \in l^{\times}$(voir [36, Lem. 3.6]), les éléments $\left\{e_{m}\right\}$ et $m_{\epsilon}$ dans $\mathrm{K}_{0}^{M W}(k)$ agissent de la même manière sur $\mathrm{K}_{1}^{M W}(l)$. Pour prouver l'égalité $\left\{e_{m}\right\}=m_{\epsilon}$, il suffit donc de trouver une extension $l / k$ telle que $\mathrm{K}_{0}^{M W}(k)$ agit fidèlement sur $\mathrm{K}_{1}^{M W}(l)$. L'extension $l=k(t)$, avec $t$ une indéterminée, convient. En effet, d'après [36, Th. 3.15], $\mathrm{K}_{1}^{M W}(k(t))$ contient une copie de $\mathrm{K}_{0}^{M W}(k)$ qui est fournie par les deux applications inverses l'une de l'autre

$$
[t] \cdot-: \mathrm{K}_{0}^{M W}(k) \longrightarrow \mathrm{K}_{1}^{M W}(k(t)) \quad \text { et } \quad \partial_{v}^{t}: \mathrm{K}_{1}^{M W}(k(t)) \longrightarrow \mathrm{K}_{0}^{M W}(k)
$$

avec $v$ la valuation $t$-adique de $k(t)$.

Remarque C.10 - Pour la preuve de la Proposition C.5, nous avons besoin d'une petite partie des travaux de F. Morel $[\mathbf{3 2}, \mathbf{3 3}, \mathbf{3 6}]$. En fait, quitte à travailler un plus, on peut vraisemblablement remplacer l'utilisation de $[\mathbf{3 2}, \mathbf{3 3}, \mathbf{3 6}]$ par les résultats très concrets et relativement élémentaires de Cazanave $[\mathbf{1 0}]$ sur le calcul de l'ensemble $\left[\mathbb{P}^{1}, \mathbb{P}^{1}\right]^{\mathrm{N}}$ des classes d'homotopie naïve d'endomorphismes de la droite projective pointée.

\section{Références}

[1] M. Artin, A. Grothendieck et J.-L. Verdier. - Théorie des topos et cohomologie étale des schémas, tome 1. Séminaire de Géométrie Algébrique du Bois-Marie 1963-1964. Lecture notes in mathematics 269. Springer-Verlag 1972. 
[2] M. Artin, A. Grothendieck et J.-L. Verdier. - Théorie des topos et cohomologie étale des schémas, tome 2. Séminaire de Géométrie Algébrique du Bois-Marie 1963-1964. Lecture notes in mathematics 270. Springer-Verlag 1972.

[3] M. Artin, A. Grothendieck. et J.-L. Verdier. - Théorie des topos et cohomologie étale des schémas, tome 3. Séminaire de Géométrie Algébrique du Bois-Marie 1963-1964. Lecture notes in mathematics 305. Springer-Verlag 1972.

[4] J. Ayoub. - Les six opérations de Grothendieck et le formalisme des cycles évanescents dans le monte motivique I. Astérisque 314 (2007).

[5] J. Ayouв. - Les six opérations de Grothendieck et le formalisme des cycles évanescents dans le monte motivique II. Astérisque 315 (2007).

[6] J. Ayoub. - Motifs de variétés analytiques rigides. Preprint (2008), disponible sur http:// www . math. uiuc . edu/K-theory/0907.

[7] J. Ayoub. - Note sur les opérations de Grothendieck et la réalisation de Betti. Journal of the Inst. of Math. Jussieu (2010) 9(2), p. 225-263.

[8] J. Ayouв. - L'Algèbre de Hopf et le groupe de Galois motiviques d'un corps de caractéristique nulle, I. Preprint (2010). To appear in Crelle's Journal.

[9] P. Balmer AND M. Schlichting. - Idempotent completion of triangulated categories. J. Algebra 236 (2001), no. 2, p. 819-834.

[10] C. Cazanave. - Algebraic homotopy classes of rational functions. Annales Scientifiques de l'École Normale Supérieure, $4^{\mathrm{e}}$ série, tome 45, 2012, p. 511-534.

[11] D.-C. Cisinski And F. DÉGlise. — Triangulated categories of mixed motives. Preprint 2009.

[12] P. Deligne et N. Katz. - Groupes de monodromie en géométrie algébrique, tome 2. Séminaire de Géométrie Algébrique du Bois-Marie 1967-1969. Lecture Notes in Mathematics 340. SpringerVerlag 1973.

[13] T. Ekedahl. - On the adic formalism. The Grothendieck Festschrift, Vol. II, 197-218, Progr. Math., 87, Birkhäuser Boston, Boston, MA, 1990.

[14] O. GABBER. - Finiteness theorems for étale cohomology of excellent schemes. Conference in honor of P. Deligne on the occasion of his 61st birthday, IAS, Princeton, October 2005. Notes disponibles sur http://www . math. polytechnique. fr/ laszlo/gdtgabber/abelien.pdf.

[15] D. GePNER AND V. SNAITH. - On the motivic spectra representing algebraic cobordism and algebraic K-theory. Documenta Mathematica 14 (2009) p. 359-396.

[16] P. G. Goerss and J. F. Jardine. - Simplicial homotopy theory. Progress in Mathematics, 174. Birkhäuser Verlag, Basel, 1999. xvi+510 pp.

[17] D. Grayson. - Higher algebraic K-theory. II (after Daniel Quillen). In "Algebraic K-theory" (Proc. Conf., Northwestern Univ., Evanston, Ill., 1976), pp. 217-240. Lecture Notes in Math., Vol. 551, Springer, Berlin, 1976.

[18] A. Grothendieck, M. Raynaud et D.-S. Rim. - Groupes de monodromie en géométrie algébrique, tome 1. Séminaire de Géométrie Algébrique du Bois-Marie 1967-1969. Lecture Notes in Mathematics 288. Springer-Verlag 1973.

[19] H. HiRonaka. - Resolution of singularities of an algebraic variety over a field of characteristic zero, I, II. Annals of Mathematics (2) 79 (1964), pp. 109-326.

[20] M. HoveY. - Spectra and symmetric spectra in general model categories. J. Pure Appl. Algebra 165 (2001), no. 1, p. 63-127.

[21] M. Hovey, B. Shipley and J. Sмith. - Symmetric spectra. J. Amer. Math. Soc. 13 (2000), no. 1, p. 149-208.

[22] A. Huber. - Realization of Voevodsky's motives. J. Algebraic Geom. 9 (2000), no. 4, p. 755-799.

[23] A. Huber. - Corrigendum to « Realization of Voevodsky's motives ». J. Algebraic Geom. 13 (2004), no. 1, p. 195-207.

[24] L. IllusiE. - On Gabber's uniformization theorems : outline and applications to étale cohomology. Conference on Ramification and vanishing cycles, University of Tokyo, Sept. 11, 2007. Notes disponibles sur http://www . math.u-psud.fr/ illusie/Illusie_Tokyo.pdf. 
[25] L. Illusie, Y. Laszlo et F. Orgogozo. - Travaux de Gabber sur l'uniformisation locale et la cohomologie étale des schémas quasi-excellents. Séminaire à l'École Polytechnique, 2006-2008. Livre disponible sur http: //www . math. polytechnique.fr/ orgogozo/travaux_de_Gabber/GTG /GTG.pdf.

[26] F. Ivorra. - Réalisation -adique des motifs triangulés géométriques. I. Doc. Math. 12 (2007), 607-671.

[27] J. F. JARDINE. - Presheaves of chain complexes. Special issue in honor of Hyman Bass on his seventieth birthday. Part IV. K-Theory 30 (2003), no. 4, 365-420.

[28] J. F. JARDINE. — Motivic symmetric spectra. Doc. Math. 5 (2000), p. 445-553.

[29] A. J. DE Jong. —Smoothness, semi-stability and alterations. Publications mathématiques de l'I.H.É.S., tomes 83 (1996), p. 51-93.

[30] M. Levine. - Motivic tubular neighborhoods. Doc. Math. 12 (2007) p. 71-146.

[31] C. Mazza, V. Voevodsky and C. Weibel. - Lecture notes on motivic cohomology. Clay Mathematics Monographs, vol. 2, 2006.

[32] F. Morel. - An introduction to $\mathbb{A}^{1}$-homotopy theory. In "Contemporary developments in algebraic $K$-theory”, ICTP Lecture notes, 15 (2003), pp. 357-441, M. Karoubi, A.O. Kuku , C. Pedrini (ed.).

[33] F. Morel. - On the motivic stable $\pi_{0}$ of the sphere spectrum. In Axiomatic, Enriched and Motivic Homotopy Theory, pp. 219-260, J. P. C. Greenlees (ed.), 2004 Kluwer Academic Publishers.

[34] F. Morel. - The stable $\mathbb{A}^{1}$-connectivity theorems. $K$-theory Journal, 2005, vol. 35, pp. 1-68.

[35] F. Morel. - Rational stable splitting of Grassmanians and the rational motivic sphere spectrum. Preprint, 2006.

[36] F. Morel. - $\mathbb{A}^{1}$-Algebraic topology over a field. Lecture Notes in Mathematics 2052, Springer Verlag.

[37] F. Morel and V. Voevodsky. - $\mathbb{A}^{1}$-Homotopy theory of schemes. Publications Mathématiques de l'I.H.E.S. (1999), volume 90, pp. 45-143.

[38] D. Quillen. - Higher algebraic K-theory. I. In "Algebraic K-theory, I : Higher K-theories", (Proc. Conf., Battelle Memorial Inst., Seattle, Wash., 1972), pp. 85-147. Lecture Notes in Math., Vol. 341, Springer, Berlin 1973.

[39] J. Riou. - Dualité de Spanier-Whitehead en géométrie algébrique. Comptes Rendus Mathématiques, Académie des Sciences, Paris, Série I 340 (2005), pages 431-436.

[40] J. Riou. - Algebraic K-theory, $\mathbb{A}^{1}$-homotopy and Riemann-Roch theorems. Journal of Topology 3 , p. 229-264, (2010).

[41] O. Röndigs and P.-A. Østvær. - Motives and modules over motivic cohomology. C. R. Acad. Sci. Paris, Ser. I 342 (2006) 751-754.

[42] O. Röndigs And P.-A. Østver. — Modules over motivic cohomology. Advances in Mathematics 219 (2008), pp. 689-727.

[43] O. Röndigs and P.-A. Østvar. - Rigidity in motivic homotopy theory. Mathematische Annalen (2008) 341, pp. 651-675.

[44] J.-P. Serre. - Cohomologie Galoisienne. Cinquième édition, révisée et complétée. Lecture Notes in Mathematics 5, Springer Verlag 1997.

[45] M. SPITZWECK AND P.-A. ØstV aR. - The Bott inverted infinite projective space is homotopy algebraic K-theory. Bulletin of the London Mathematical Society 41(2) (2009) p. 281-292.

[46] A. Suslin AND V. Voevodsky. - Singular homology of abstract algebraic varieties. Inventiones Mathematicae (1996) 123, pp. 61-94.

[47] M. Suzuki. - Group theory I. Grundlehren der Mathematischen Wissenschaften, 247, Berlin, New York, Springer-Verlag (1982).

[48] R. Thomason and T. Trobaugh. - Higher algebraic K-theory of schemes and of derived categories, in "The Grothendieck Festschrift" : a collection of articles written in honor of the 60th birthday of Alexander Grothendieck. Volume III, Progr. Math., vol. 88, Birkhaüser, 1990, p. 247-436. 
[49] V. Voevodsky. - Triangulated categories of motives over a field. In "Cycles, transfers, and motivic homology theories", Annals of Mathematics Studies, vol. 143, Princeton University Press, 2000.

[50] V. Voevodsky. $-\mathbb{A}^{1}$-Homotopy theory. Documenta Mathematica, Extra Volume ICM (1998), I, p. 579-604.

[51] V. Voevodsky. - Cancellation theorem. Documenta Mathematica, Extra Volume Suslin (2010), p. 671-685.

[52] C. A. WeIBel. - An introduction to homological algebra. Cambridge Studies in Advanced Mathematics, 38. Cambridge University Press, Cambridge, 1994. xiv+450 pp.

[53] C. A. Weibel. - The K-book : an introduction to algebraic K-theory. Graduate Studies in Math. 145, Amer. Math. Soc., 2013.

Joseph Ayoub,

Institut für Mathematik, Universität Zürich, Winterthurerstr. 190, CH-8057 Zürich, Switzerland

CNRS, LAGA, Université Paris 13, 99 avenue J.-B. Clément, 93430 Villetaneuse, France

- E-mail : joseph.ayoub@math.uzh.ch 